\title{
CIN85 promotes proximal and distant B cell antigen receptor signaling
}

\section{Doctoral Thesis}

In partial fulfillment of the requirements for the degree

"Doctor rerum naturalium (Dr. rer. nat.)"

in the Molecular Medicine Study Program

at the Georg-August University Göttingen

\author{
submitted by \\ Kathrin Schulz \\ born in \\ Winsen/Luhe
}

Göttingen, December 2015 
Members of the thesis committee:

\section{Supervisor:}

Prof. Dr. Jürgen Wienands

Institute of Cellular and Molecular Immunology, University Medical Center, Göttingen

Second member of the thesis committee:

Prof. Dr. Henning Urlaub

Bioanalytical Mass Spectrometry Group, Max-Planck-Institute for Biophysical Chemistry, Göttingen

Third member of the thesis committee:

Prof. Dr. Peter Schu

Department of Cellular Biochemistry, University Medical Center, Göttingen

Date of Disputation: 


\section{Affidavit}

Here, I declare that my doctoral thesis entitled "CIN85 promotes proximal and distant B cell antigen receptor signaling" has been written independently with no other sources and aids than quoted.

Kathrin Schulz

Göttingen,

December

2015 
List of publications

2015 Julius Kühn, Leo Wong, Sona Pirkuliyeva, Kathrin Schulz, Claudia Schwiegk, Kevser Gencalp, Selina Keppler, Facundo D. Batista, Henning Urlaub, Michael Habeck, Stefan Becker, Christian Griesinger, Jürgen Wienands

CIN85 triggers formation of intracellular signaling clusters for B cell activation

Science Signaling, in revision

Participation at scientific meetings:

2012 Oral presentation, $4^{\text {th }}$ DGfl Autumn School Current Concepts in Immunology, Bad Schandau

2013 Poster presentation, $9^{\text {th }}$ DGfl Spring School on Immunology, Ettal

2015 Poster presentation, $13^{\text {th }}$ B Cell Forum, Hitzacker

2015 Poster presentation (poster prize), $4^{\text {th }}$ European Congress of Immunology, Vienna 


\section{TABLE OF CONTENTS}

Affidavit

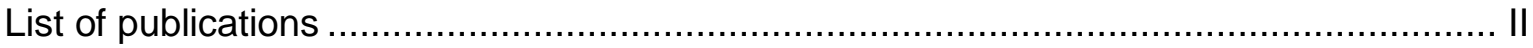

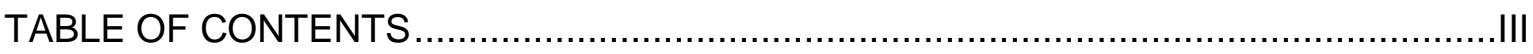

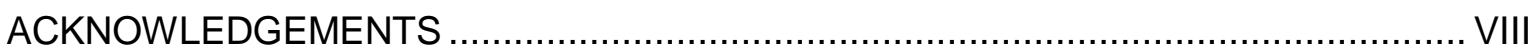

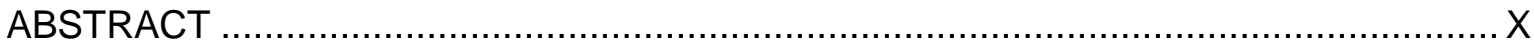

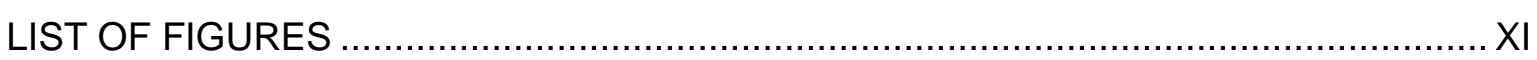

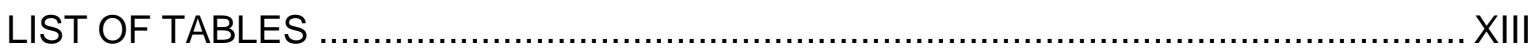

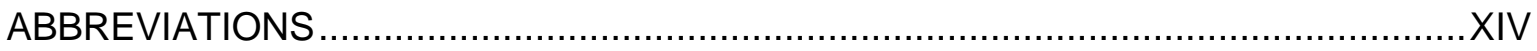

AMINO ACIDS AND DEOXYRIBONUCLEOTIDES ..............................................XIX

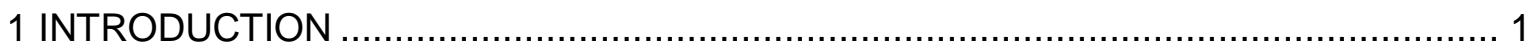

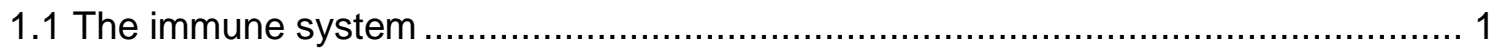

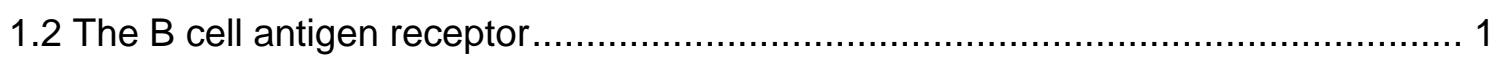

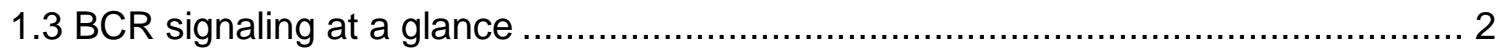

1.4 SLP65 function depends on the support of its constitutive interactor CIN85 ............ 4

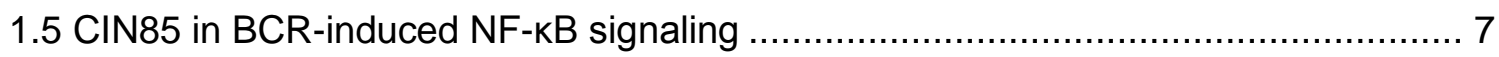

1.6 CIN85 orchestrates signaling pathways downstream of several receptors..............10

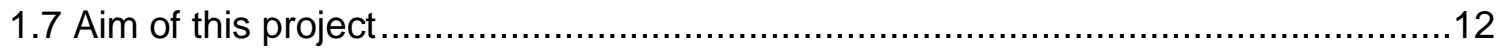

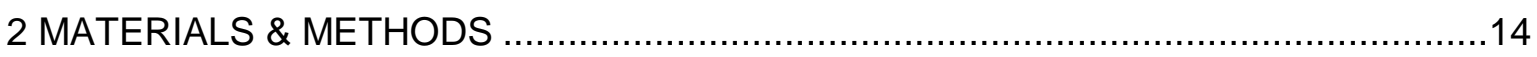

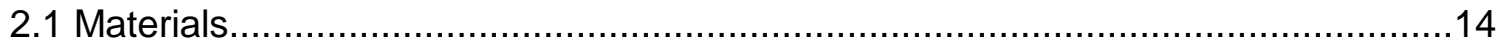

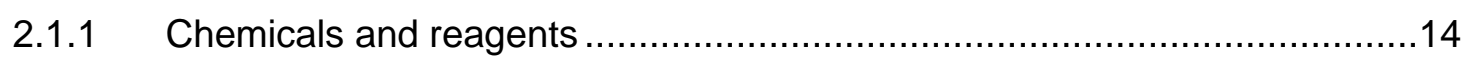

2.1.2 Consumable goods..................................................................... 14

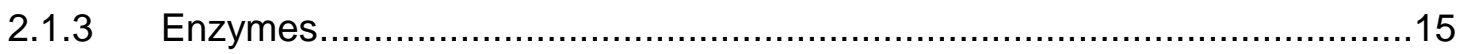

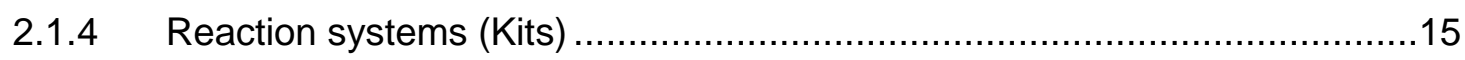

2.1.5 Synthetic DNA oligonucleotides .........................................................16

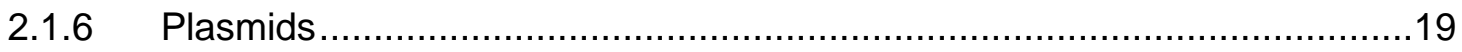

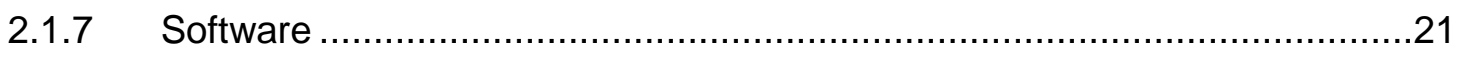

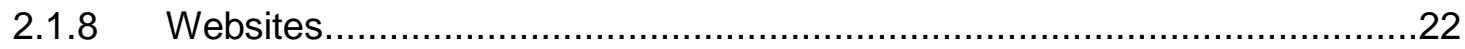

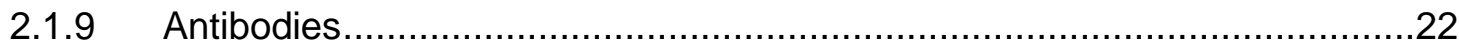




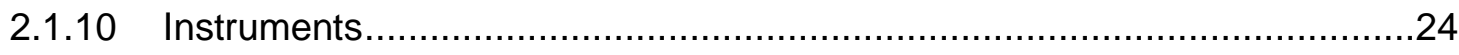

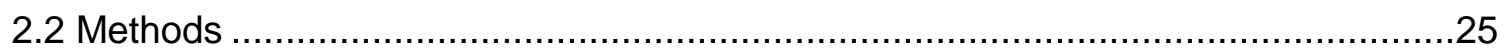

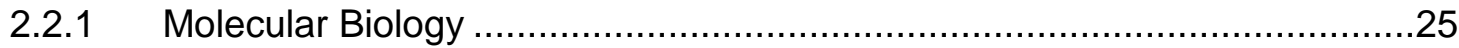

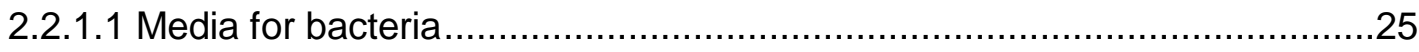

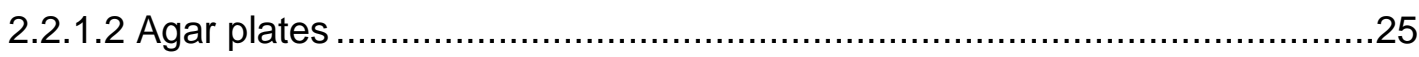

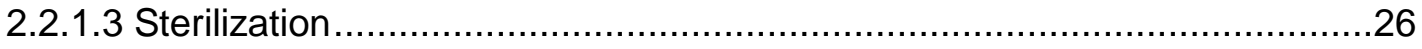

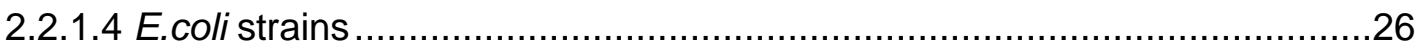

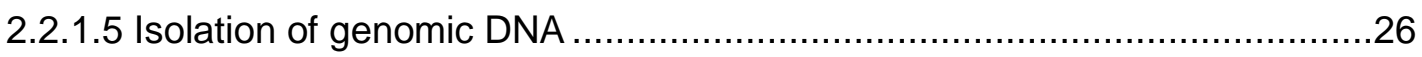

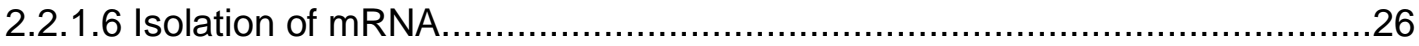

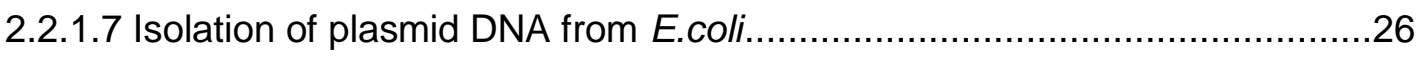

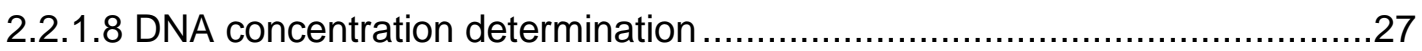

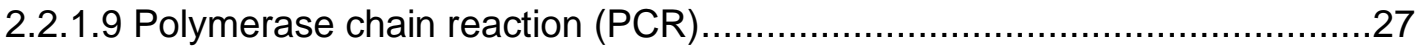

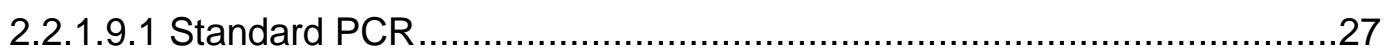

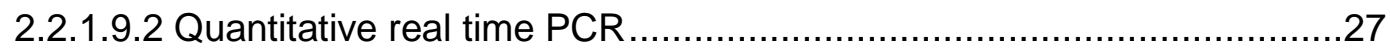

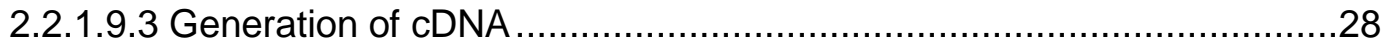

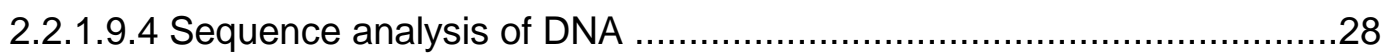

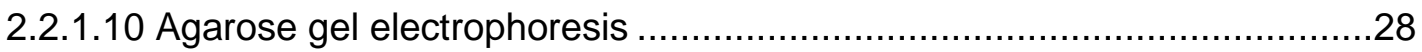

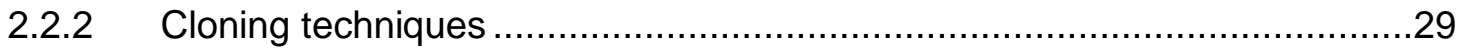

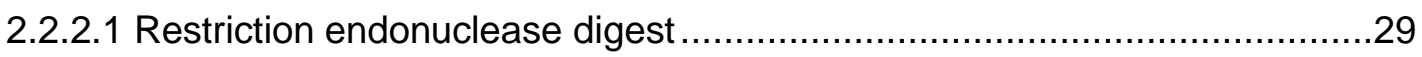

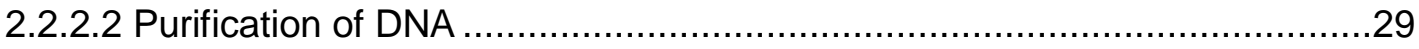

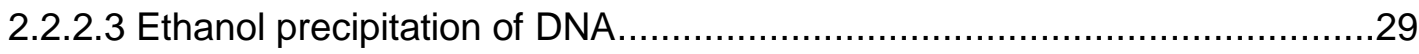

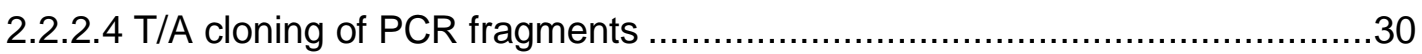

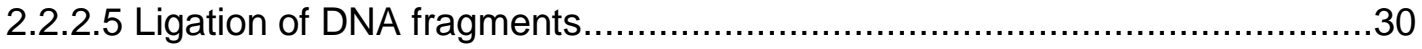

2.2.2.6 Preparation of chemically competent E.coli .......................................30

2.2.2.7 Transformation of chemically competent E.coli..................................30

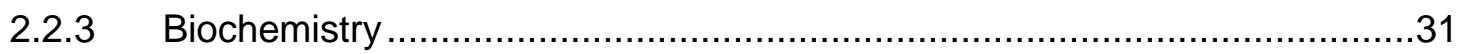

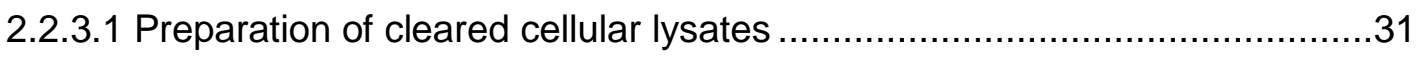

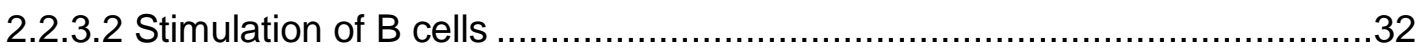

2.2.3.3 Expression and purification of recombinant GST-fusion proteins ...............32

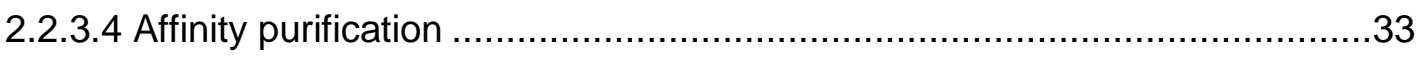


2.2.3.5 SDS-polyacrylamide gel electrophoresis ...........................................3

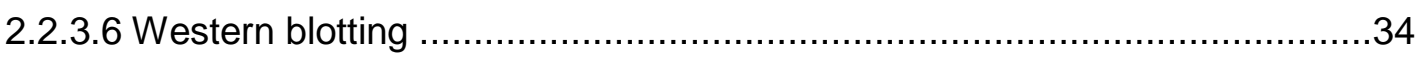

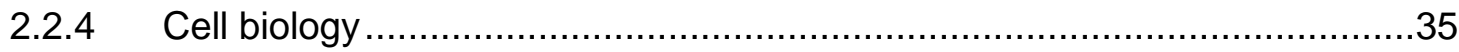

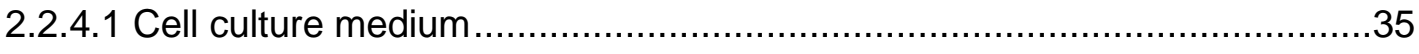

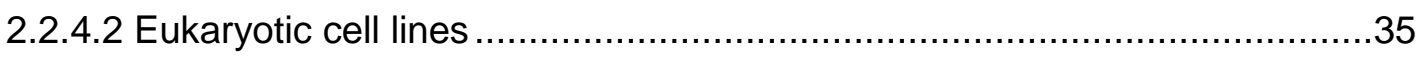

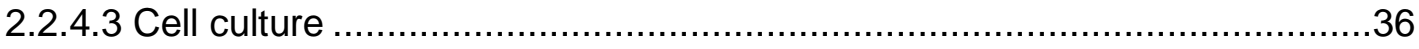

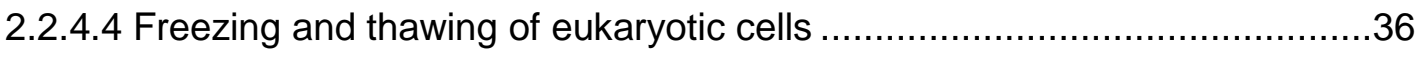

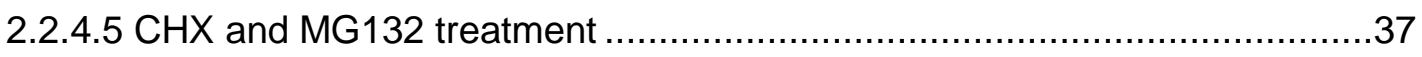

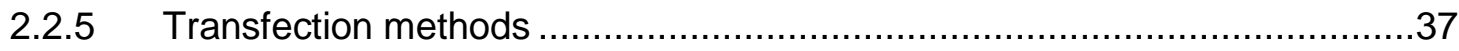

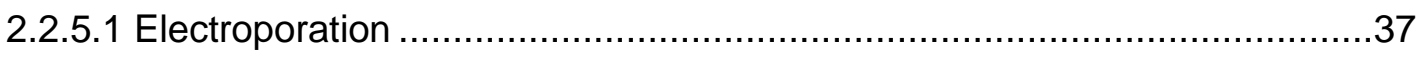

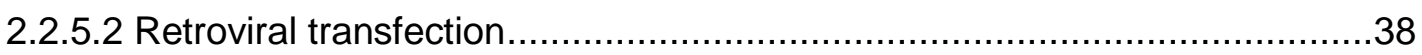

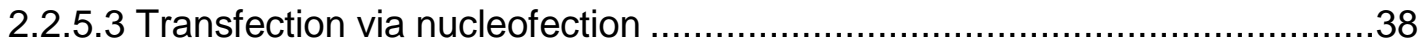

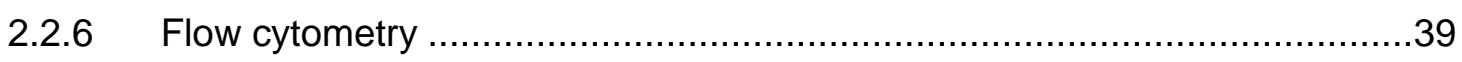

2.2.6.1 Analysis of fluorophore expression .........................................................39

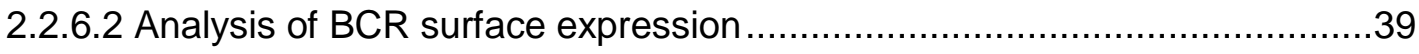

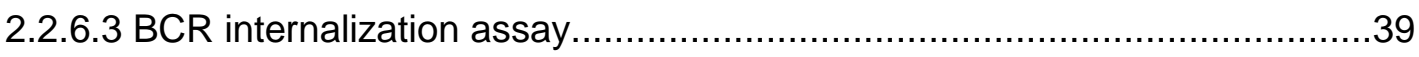

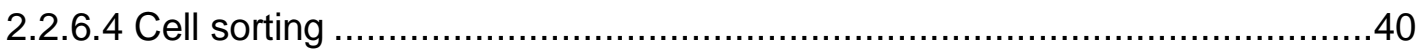

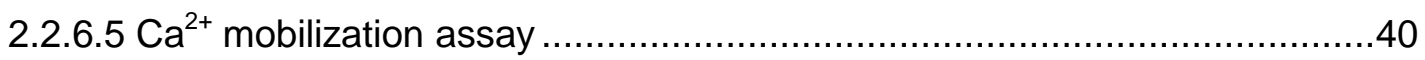

2.2.7 Confocal laser scanning microscopy................................................40

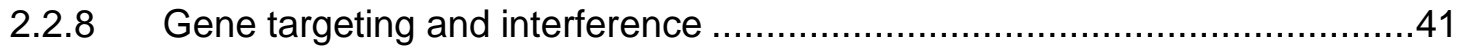

2.2.8.1 RNA interference with microRNA-30 adapted shRNA-mir retroviral vector 41

2.2.8.2 Transcription Activator-like Effector Nucleases - TALEN .........................41

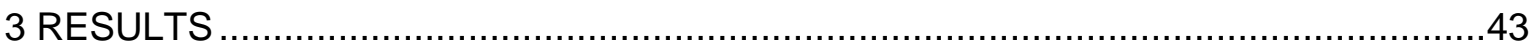

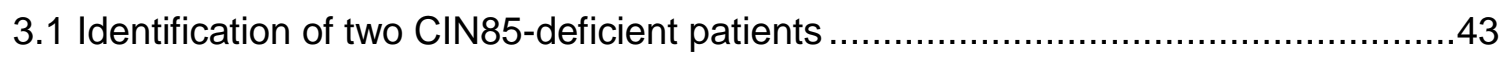

3.2 Human CIN85 regulates proximal BCR signaling .........................................44

3.2.1 Transcription Activator-like Effector Nucleases- (TALEN-) mediated CIN85 inactivation in the human B cell line DG75 .........................................44

3.2.2 Tyrosine phosphorylation events upon BCR stimulation are not affected by the loss of CIN85 expression ...........................................................46

3.2.3 Loss of CIN85 moderately impairs BCR-mediated $\mathrm{Ca}^{2+}$ signaling ..............47 
3.2.4 Both $\mathrm{SH} 3$ and coiled coil domains are mandatory for membrane recruitment of CIN85 and $\mathrm{Ca}^{2+}$ signaling .49

3.2.5 Membrane recruitment of CIN85 is not influenced by Lyn interaction..........50

3.2.6 CIN85 positively regulates the function of SLP65 .51

3.3 CIN85 regulates the function of SLP65 in BCR-induced $\mathrm{Ca}^{2+}$ signaling 53

3.3.1 Generation of a TALEN-mediated CIN85/SLP65 double-deficient DG75 subline. .53

3.3.2 Plasma membrane recruitment of SLP65 is supported by CIN85 56

3.4 CIN85 mediates SLP65 protein stability .57

3.4.1 SLP65 protein expression is reduced in CIN85-deficient DG75 cells .57

3.4.2 Protein degradation of SLP65 is prevented by CIN85 ...........................58

3.4.3 CIN85's stabilization of SLP65 is independent of $\mathrm{Ca}^{2+}$ mobilization............60

3.5 CIN85 interacts with the short cytoplasmic tail of the IgM-BCR .61

3.5.1 Colocalization of CIN85, SLP65 and the BCR upon BCR stimulation ........61

3.5.2 CIN85 interacts with the cytoplasmic part of the IgM-BCR heavy chain ......63

3.5.3 The cytoplasmic part of the IgM heavy chain is ubiquitinylated upon BCR stimulation

3.5.4 CIN85 interacts via its SH3 domains with ubiquitin

3.5.5 IgM heavy chain ubiquitinylation enhances CIN85 interaction with the BCR ..

3.5.6 BCR-induced tyrosine phosphorylation and $\mathrm{Ca}^{2+}$ response are independent of KVK .71

3.5.7 BCR internalization is not influenced by the KVK cytosolic part of the BCR heavy chain and CIN85 .73

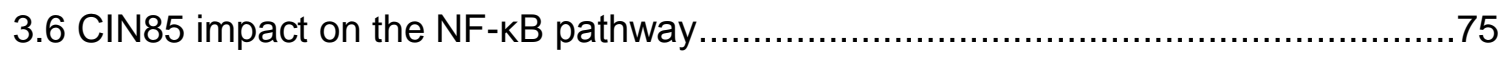

3.6.1 Enhancing function of CIN85 in the NF-KB pathway...............................75

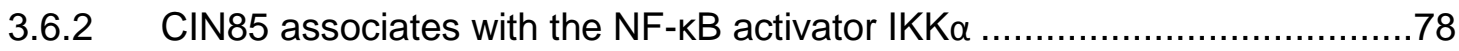

3.6.3 CIN85 interacts stimulation dependent with $\mathrm{PKC} \beta$ and influences its

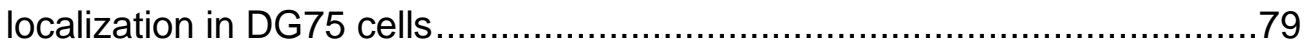

3.6.4 Plasma membrane localization of PKC $\beta$ II is regulated by CIN85 ...............81

3.6.5 CIN85 binds to Rhotekin2 (RTKN2) with its SH3 domains .......................83 
3.6.6 Chicken RTKN2 acts as activating regulator of $\mathrm{Ca}^{2+}$ and NF-KB signaling in $B$ cells

3.6.7 RTKN2 neither acts in $\mathrm{Ca}^{2+}$ mobilization nor in NF-KB activation in human $\mathrm{B}$ cells.....

3.6.8 Protein stability of the ubiquitously localized RTKN2 depends on CIN85 ....89 4 DISCUSSION .92

4.1 CIN85 fine tunes BCR-induced $\mathrm{Ca}^{2+}$ mobilization .92

4.2 CIN85 acts in BCR proximal events. 95

4.2.1 The kinetic of SLP65 plasma membrane translocation depends on CIN85 in human B cells.... .95

4.2.2 CIN85 is directly recruited into BCR signalosomes .96

4.2.3 Ubiquitinylation events calibrate CIN85's BCR-proximal interaction network.

4.2.4 The cytoplasmic tail of the mlgM determines BCR complex stability..... .98

4.2.5 Internalization of antigen-bound BCR does not depend on the BCR's cytoplasmic region .99

4.3 Identification of a new CIN85 function: protein stabilization 100

4.4 BCR-induced NF-KB signaling is regulated by CIN85 101

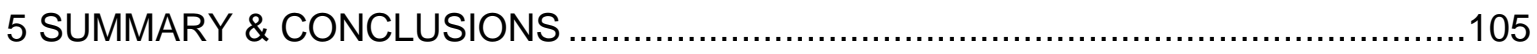

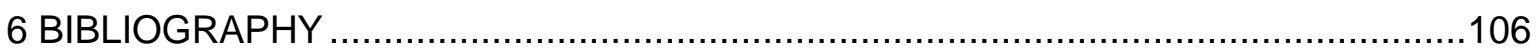

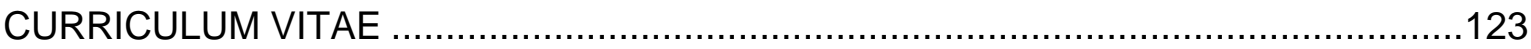




\section{ACKNOWLEDGEMENTS}

I would like to express my gratitude to Prof. Dr. Jürgen Wienands for giving me the opportunity to conduct my $\mathrm{PhD}$ thesis project in his group and for providing me with support, motivating words when needed, advice and also the opportunity to develop and follow my own ideas.

I also want to acknowledge the members of my thesis committee, Prof. Dr. Henning Urlaub and Prof. Dr. Uwe Hanisch for their advice during all the thesis committees. In addition, I want to thank Prof. Dr. Peter Schu for his commitment in the end of my PhD project, since he compensated the regrettable loss of Prof. Dr. Uwe Hanisch.

Furthermore, I want to thank the Molecular Medicine Study Programm. I am also grateful for the financial support by the Medical Faculty of the Georg-August University of Göttingen and the SFB860.

I also want to thank Prof. Dr. Carsten Lüder for providing me with access to the confocal microscope in the Medical Microbiology department and Sabrina for passing time with me during cell sorting processes.

I am very thankful to Gabriele Sonntag and Ines Heine for running the lab and providing not only essential supplies and technical assistance but also filling the lab with a comfortable atmosphere. Along these lines, I also want to thank "the two" from the secretary, Anika Schindler and Ingrid Teuteberg, for always helping with organizational businesses.

I also want to thank my students of these particularly Lena and Alex contributed to this project.

Special thanks to Christoffer, Kai, Caren, Henrike and Niklas for proofreading my thesis and to Christoffer for helping me with the formatting.

Thanks to the former and current members from the Institute of Cellular and Molecular Immunology for giving me a good time. Special thanks to Christoffer, Caren, Sona, Kanika, Henrike, Wiebke, Vanessa, Kai, Julius, Niklas, Johannes and Michael who shared not only scientific discussions but also lunch at the Mensa and/or coffee breaks. We also did activities outside of the lab, which I really enjoyed. 
I also want to thank Linda and Franzi for their friendship and support for such a long time. I was lucky to find myself in the same town like you, Linda, so that I always had a piece of home close by me and someone who is always there.

Finally, I want to thank my parents and my brother, who always supported me and made my life's journey possible so far. Dear Christoffer, I am so thankful that you accompanied me during the last years. We overcame the downs and enjoyed the ups together. I look forward to the things that are waiting for us. 


\section{ABSTRACT}

$B$ cell antigen receptor (BCR) signaling is pivotal in B cell development and initiation of humoral immune responses. In this process, numerous proteins are involved that interact with each other and build up a sophisticated interplay of activating as well as terminating complexes to generate an appropriate signal outcome. Recently, two boys were identified who showed deficiencies for serum immunoglobulins leading to recurrent infections due to a big deletion in the gene coding for the adaptor protein CIN85 (Cbl-interacting protein of $85 \mathrm{kDa}$ ). CIN85 is known to be a constitutive interactor of the adaptor protein SLP65 (SH2 domain-containing leukocyte protein of $65 \mathrm{kDa}$ ) and supports the plasma membrane recruitment of SLP65 as a platform for the assembly of the $\mathrm{Ca}^{2+}$ initiation complex in the chicken DT40 B cell line. Furthermore, involvement of CIN85 is reported in the activation of the BCR-induced canonical NF-KB (nuclear factor 'kappa-light-chain-enhancer' of activated B-cells) pathway in primary mouse B cells. However, the precise mechanism of CIN85 plasma membrane recruitment and hence provided support for SLP65 function as well as NF-KB activation is unknown. Here I show that CIN85 regulates plasma membrane recruitment of SLP65 and the known canonical NF-kB pathway activator PKC $\beta$ (Protein kinase $C$ beta) in the human DG75 B cell line. Based on the generation of a human CIN85-deficient B cell sub-line, I found that CIN85 supports BCR-induced SLP65 membrane recruitment probably due to direct and/or ubiquitin mediated interaction with proteins present in BCR signalosomes as well as by direct association with the short cytoplasmic tail of the IgM-BCR. With confocal microscopy, I could provide evidence for the importance of CIN85 in respect to PKC $\beta$ localization and hence narrow the point of CIN85 interaction in enhancing BCR-induced NF-kB signaling. Moreover, I could show for the first time that CIN85 acts as a stabilizer of the direct $\mathrm{SH} 3$ domain interacting protein SLP65. Collectively, my results demonstrate that CIN85 exerts diverse roles in different stages of BCR-induced signaling and hence highlight the importance of this adaptor protein. Thus, the results of my $\mathrm{PhD}$ project contribute to a better understanding of the complex BCR signaling cascade. Noteworthy, the performance of genetic and biochemical experiments in the generated model systems provide further insights into the mechanistic basis of the patients phenotype and at the same time open new opportunities for future investigations. 


\section{LIST OF FIGURES}

Figure 1.1: BCR-induced signal transduction leads to cytosolic enrichment of the second

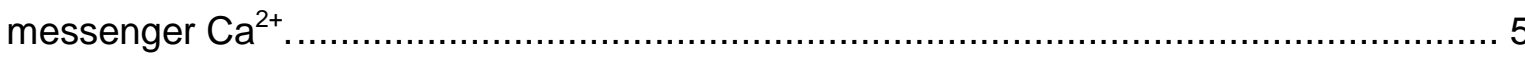

Figure 1.2: SLP65 and CIN85 domain architecture and interaction. ............................. 6

Figure 1.3: BCR-mediated signaling in the context of NF-kB activation.......................... 9

Figure 3.1: Identification of a CIN85-deficient patient... Fehler! Textmarke nicht definiert.

Figure 3.2: TALEN-mediated loss of CIN85 in DG75 B cells.

Figure 3.3: Loss of CIN85 does not influence BCR-mediated tyrosine phosphorylation

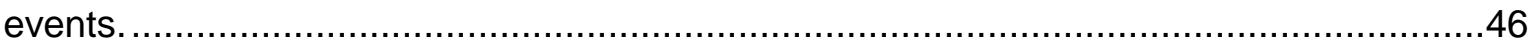

Figure 3.4: CIN85 deficiency leads to reduced $\mathrm{Ca}^{2+}$ signaling upon BCR activation.........48

Figure 3.5: Truncated CIN85 variants are not sufficient to enable complete BCR signaling.

Figure 3.6: CIN85-Lyn interaction does not mediate CIN85 plasma membrane recruitment.

Figure 3.7: CIN85 interaction with SLP65 positively modulates SLP65 function in $\mathrm{Ca}^{2+}$ flux

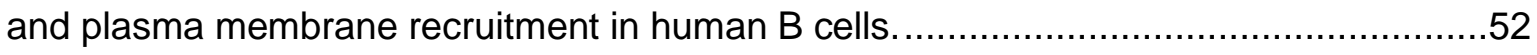

Figure 3.8: Generation of CIN85/SLP65 double-deficient DG75 cells. ............................53

Figure 3.9: CIN85 and SLP65 act in concert to enhance BCR-induced $\mathrm{Ca}^{2+}$ mobilization.

Figure 3.10: CIN85 enhances SLP65 plasma membrane recruitment............................57

Figure 3.11: SLP65 expression is diminished in CIN85-deficient DG75 cells. ..................58

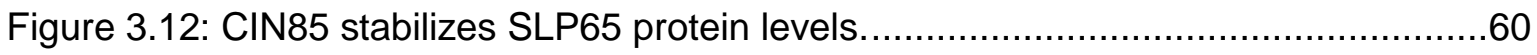

Figure 3.13: SLP65-independent influence on $\mathrm{Ca}^{2+}$ flux of CIN85 ..................................61

Figure 3.14: CIN85, SLP65 and the BCR colocalize after BCR activation......................63

Figure 3.15: CIN85 associates with the $\lg M-B C R$ independent of the $\lg \alpha / \lg \beta$ heterodimer.

Figure 3.16: The amino acid sequence KVK in the cytoplasmic tail of the BCR is

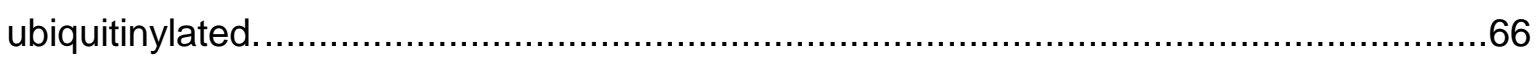

Figure 3.17: CIN85 can interact with ubiquitin.

Figure 3.18: Ubiquitinylation of the BCR influences its interaction with CIN85 but is negligible for CIN85 plasma membrane recruitment.

Figure 3.19: The cytoplasmic amino acids KVK of the BCR heavy chain did not reveal signaling capability in terms of tyrosine phosphorylation and $\mathrm{Ca}^{2+}$ mobilization..... .72

Figure 3.20: The KVK sequence in the BCR heavy chain has no impact on BCR

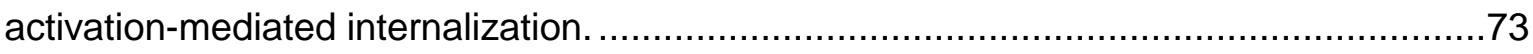

Figure 3.21: Loss of CIN85 does not alter the process of BCR internalization. .................75

Figure 3.22: Loss of CIN85 diminishes BCR-induced NF-KB activation..........................77 
Figure 3.23: CIN85 shows interaction with IKKa. ................................................. 78

Figure 3.24: PKCßII interacts with CIN85 upon BCR stimulation.................................. 80

Figure 3.25: CIN85 affects localization of PKC $\beta I I$ but not of CARMA1 and Bcl10 .............82

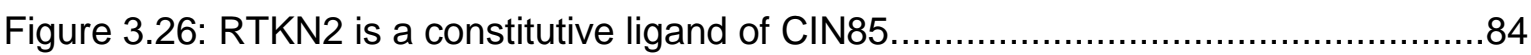

Figure 3.27: Reduced RTKN2 protein levels led to diminished NF-KB and $\mathrm{Ca}^{2+}$ signaling in DT40 cells. .86

Figure 3.28: Reduced RTKN2 protein levels in DG75 cells did not affect $\mathrm{Ca}^{2+}$ and NF-KB signaling. .89

Figure 3.29: RTKN2 is ubiquitously localized and its stability is mediated by CIN85. .......90 Figure 4.1: Schematic drawing of CIN85 involvement in BCR-induced NF-KB signaling.103 


\section{LIST OF TABLES}

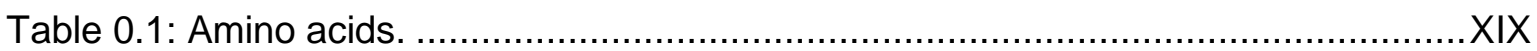

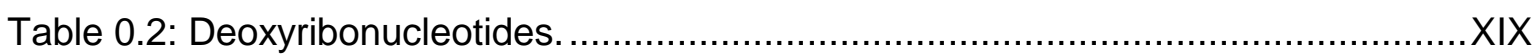

Table 2.1: Consumables used in this project................................................................14

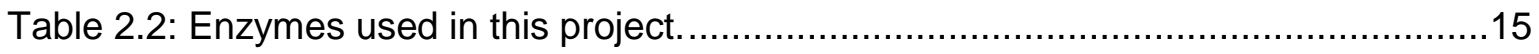

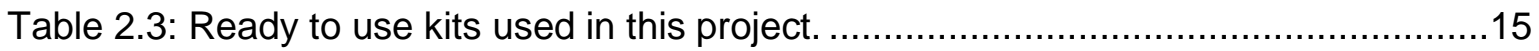

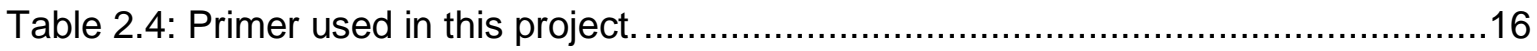

Table 2.5: Plasmids used for cloning and expression in this project...............................19

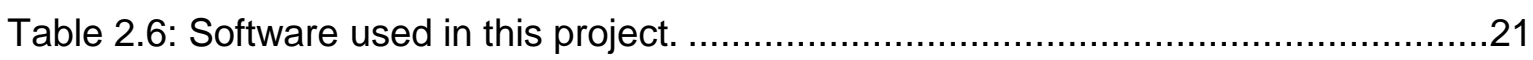

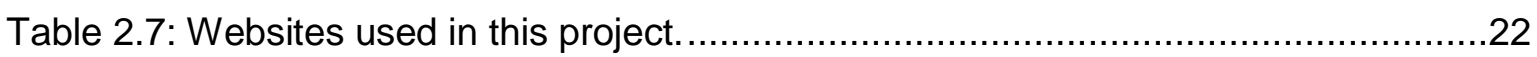

Table 2.8: Antibodies used in this project............................................................22

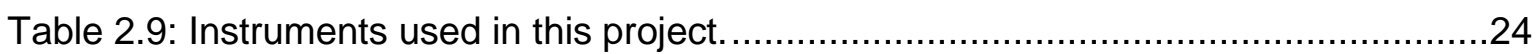

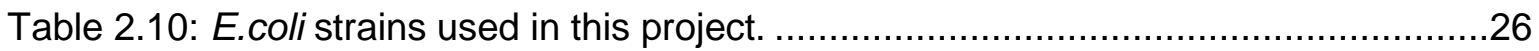

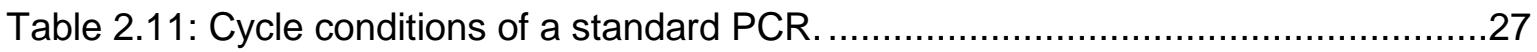

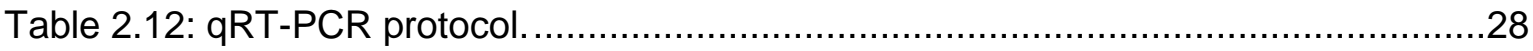

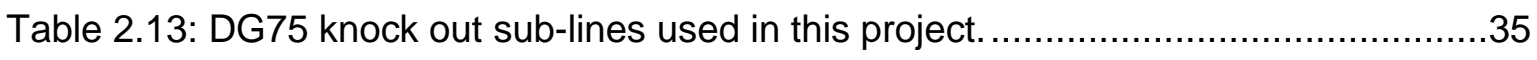

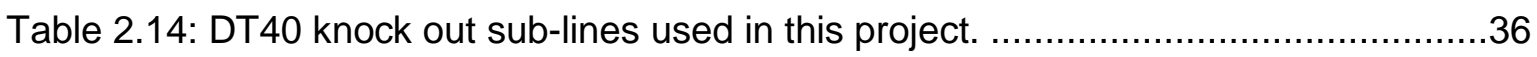




\section{ABBREVIATIONS}

\begin{tabular}{|c|c|}
\hline${ }^{\circ} \mathrm{C}$ & degree Celsius \\
\hline$\mu$ & micro \\
\hline A & ampere \\
\hline aa & amino acid \\
\hline $\mathrm{Ag}$ & antigen \\
\hline AP & affinity purification \\
\hline ATCC & American type culture condition \\
\hline ATP & adenosine triphosphate \\
\hline BAFF & $B$ cell activating factor \\
\hline BASH & $\mathrm{B}$ cell adaptor containing an $\mathrm{SH} 2$ domain \\
\hline Bcl10 & B-cell lymphoma/leukemia 10 \\
\hline BCR & B cell antigen receptor \\
\hline BD & Becton Dickinson \\
\hline blast & blasticidin resistance \\
\hline bleo & bleocin resistance \\
\hline BLNK & B cell linker \\
\hline bp & base pair \\
\hline BSA & bovine serum albumin \\
\hline Btk & Bruton's tyrosine kinase \\
\hline CARMA1 & CARD-containing MAGUK protein 1 \\
\hline cbl & Casitas B-lineage lymphoma proto-oncogene \\
\hline CC & coiled coil \\
\hline CCL & cleared cellular lysates \\
\hline CD & cluster of differentiation \\
\hline CD2AP & CD2-associated protein \\
\hline$C D 2 A P^{s h}$ & DG75 cells expressing shRNA against CD2AP mRNA \\
\hline cDNA & complementary DNA \\
\hline ch & chicken \\
\hline CIN85 & Cbl-interacting protein of $85 \mathrm{kDa}$ \\
\hline CIN85 & DT40 cells expressing shRNA against CIN85 mRNA \\
\hline Cit & Citrine \\
\hline CMS & Cas ligand with multiple $\mathrm{SH} 3$ domains \\
\hline CRAC & $\mathrm{Ca}^{2+}$ release-activated $\mathrm{Ca}^{2+}$-channel \\
\hline CS & chicken serum \\
\hline CT & Crossing threshold \\
\hline C-terminal & carboxy-terminal \\
\hline
\end{tabular}




\begin{tabular}{|c|c|}
\hline ctrl. & control \\
\hline $\mathrm{Da}$ & Dalton \\
\hline DAG & diacylglycerol \\
\hline${ }_{\mathrm{dd}} \mathrm{H}_{2} \mathrm{O}$ & double-distilled water \\
\hline DKO & CIN85/SLP65 double deficient DG75 sub-line \\
\hline DMEM & Dulbecco`s modified Eagle`s medium \\
\hline DMSO & Dimethyl sulfoxide \\
\hline DNA & deoxyribonucleinacid \\
\hline dNTP & 2'-deoxynucleoside-5'-triphosphate \\
\hline DSMZ & Deutsche Sammlung von Mikroorganismen und Zellkulturen \\
\hline E.coli & Escherichia coli \\
\hline ECL & enhanced chemical luminescence \\
\hline EDTA & ethylenediamine tetraacetic acid \\
\hline EGFP & enhanced EGFP \\
\hline EGFR & epidermal growth factor receptor \\
\hline env & envelop \\
\hline ER & endoplasmic reticulum \\
\hline$F(a b `) 2$ & bivalent antigen-binding fragment \\
\hline FACS & fluorescence-activated cell sorting \\
\hline Fc & fragment crystalline \\
\hline FCS & fetal calf serum \\
\hline FcyRlla & Low affinity immunoglobulin gamma Fc region receptor II-a \\
\hline $\mathrm{FL}$ & full length \\
\hline$g$ & gram \\
\hline gag & gene encoding p55 \\
\hline GFP & green fluorescent protein \\
\hline GST & Glutathione S-transferase \\
\hline $\mathrm{h}$ & hour \\
\hline $\mathrm{HA}$ & hemagglutinin \\
\hline HEK & human embryonic kidney \\
\hline HEPES & 4-(2-hydroxyethyl)-1-piperazineethanesulfonic acid \\
\hline HRPO & Horseradish peroxidase \\
\hline $\lg$ & immunoglobuline \\
\hline $\lg \mathrm{H}$ & Ig heavy chain \\
\hline $\lg \mathrm{L}$ & Ig light chain \\
\hline IKK & IKB kinase \\
\hline
\end{tabular}




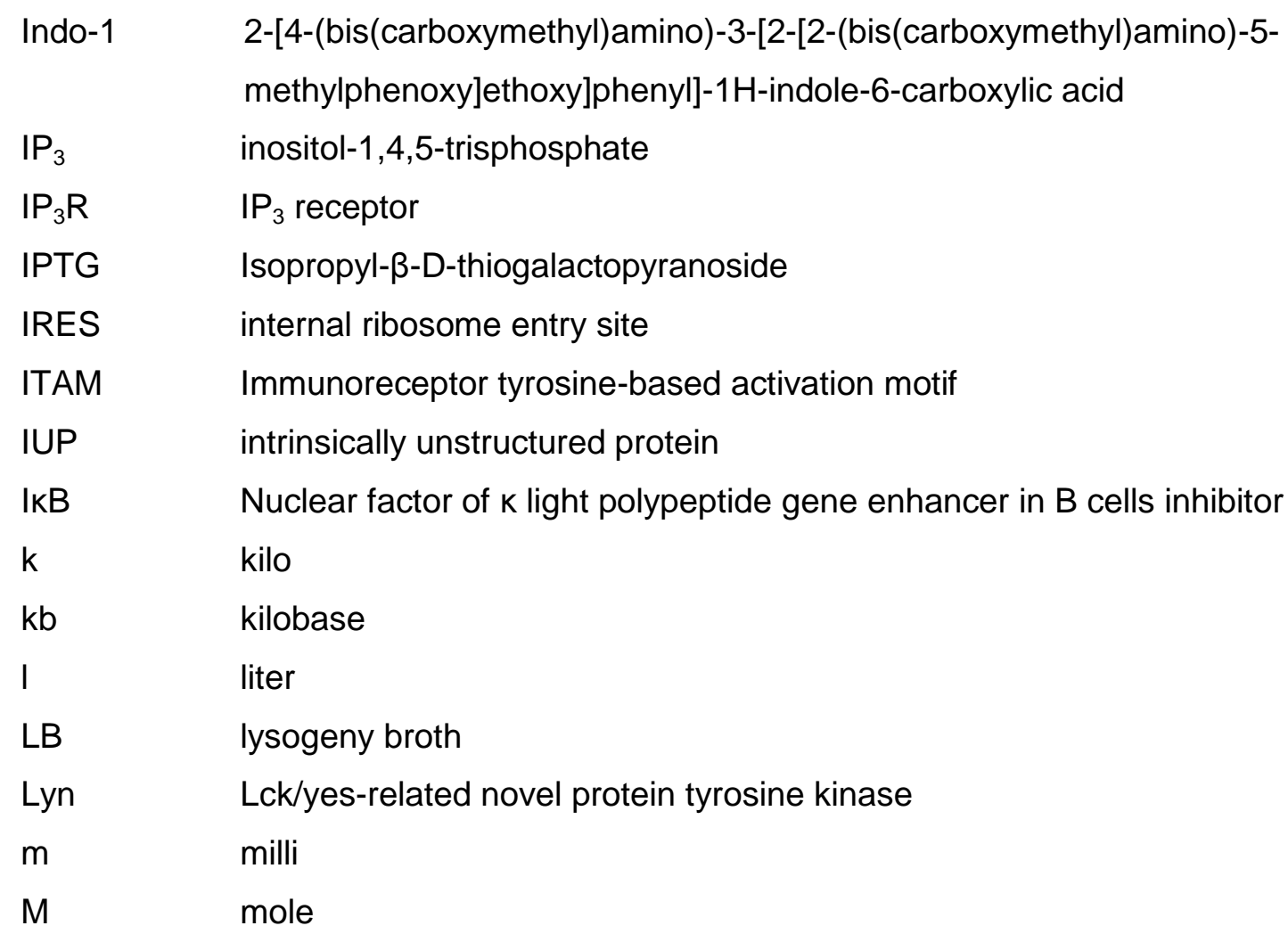

MALT1 Mucosa-associated lymphoid tissue lymphoma translocation protein 1 METS-1 mesenchyme-to-epithelium transition protein with $\mathrm{SH} 3$ domains

MFI mean fluorescence intensity

mlg membrane-bound immunoglobulin

$\min \quad$ minute

mocksh DT40 or DG75 cells expressing mock shRNA

mRNA messenger RNA

NEMO NF-kB essential modulator

NFAT Nuclear factor of activated T cells

NF-kB Nuclear factor of $\mathrm{k}$ light polypeptide gene enhancer in $\mathrm{B}$ cells

NLS Nuclear localization signals

NMR nuclear magnetic resonance spectroscopy

NP40 nonidet P40

N-terminal amino-terminal

OD optical density

P pseudosubstrate

PA Phosphatidic acid

PAGE Polyacrylamide gel electrophoresis

PBS Phosphate buffered saline

PCR polymerase chain reaction

PEST sequence rich in proline $(P)$, glutamic acid $(E)$, serine $(S)$, threonine $(T)$ 


\begin{tabular}{|c|c|}
\hline $\mathrm{PH}$ & Pleckstrin homology \\
\hline PI3K & Phosphoinositide 3'-kinase \\
\hline $\mathrm{PIP}_{2}$ & Phosphatidyl-inositol-4,5-bisphosphate \\
\hline PKC $\beta$ & Protein-kinase $\mathrm{C} \beta$ \\
\hline PlatE & Platinum-E \\
\hline PLCү2 & Phospholipase C y2 \\
\hline PMA & Phorbol myristate acetate \\
\hline pol & gene encoding protease, reverse transcriptase and integrase \\
\hline PR & proline-rich \\
\hline PRC & CIN85 proline-rich part with the coiled coil domain \\
\hline pTyr & phosphotyrosine \\
\hline puro & puromycin resistence \\
\hline pY & phosphotyrosine \\
\hline RFP & red fluorescent protein \\
\hline rpm & revolutions per minute \\
\hline RPMI & Roswell Park Memorial Institute \\
\hline RT & room temperature \\
\hline RTKN & Rhotekin \\
\hline RTKN2 & Rhotekin2 \\
\hline$R T K N 2^{s h}$ & DT40 or DG75 cells expressing shRNA against RTKN2 mRNA \\
\hline Ruk & regulator of ubiquitous kinase \\
\hline SDS & Sodium dodecylsulfate \\
\hline sec & second \\
\hline
\end{tabular}

SETA SH3 domain-containing gene expressed in tumorigenic astrocytes

$\mathrm{SH} 2 \quad$ Src-homology 2

SH3 Src-homology 3

SH3KBP1 SH3-domain kinase binding protein 1

SHIP-1 SH2 domain-containing inositol phosphatase 1

shRNA short hairpin RNA

SILAC stable isotope labeling by/with amino acids in cell culture

SLP65 SH2 domain-containing leukocyte adaptor protein of $65 \mathrm{kDa}$

SLP76 SH2 domain-containing leukocyte phosphoprotein of $76 \mathrm{kDa}$

src rous sarcoma oncogene

STIM1 stromal interaction molecule 1

sub substrate binding domain

Syk Spleen tyrosine kinase

t time 


$\begin{array}{ll}\text { TAB } & \text { TAK1 binding protein } \\ \text { TAE } & \text { Tris acetate EDTA buffer } \\ \text { TAK } & \text { Transforming growth factor beta-activated kinase } \\ \text { TALEN } & \text { transcription activator like effector nucleases } \\ \text { TBS } & \text { Tris-buffered saline } \\ \text { TBS-T } & \text { TBS 0.1\% Tween } \\ \text { TCR } & \text { T cell antigen receptor } \\ \text { TEMED } & \text { N,N,N'N-tetramethylethylene-diamine } \\ \text { TF } & \text { transcription factor } \\ \text { TI } & \text { T cell-independent } \\ \text { TIRF } & \text { total internal reflection } \\ \text { Tris } & \text { Tris-(hydroxymethyl)-aminomethane } \\ \text { TritonX-100 } & \text { polyethylene glycol p-(1,1,3,3-tetramethylbutyl)-phenyl ether } \\ \text { Tween-20 } & \text { polyoxyethylene sorbitan monolaurate } \\ \text { UV } & \text { ultraviolet } \\ \text { V } & \text { volt } \\ \text { v/v } & \text { volume per volume } \\ \text { VSV-G } & \text { vesicular stomatitis virus glycoprotein } \\ \text { w/v } & \text { weight per volume } \\ \text { wt } & \text { wild-type } \\ \text { x g } & \text { times gravity } \\ \text { X-gal } & \text { 5-bromo-4-chloro-3-indolylbeta-D-galacto-pyranoside } \\ \text { ZF } & \text { zinc finger motif } \\ \alpha & \text { anti } \\ \Delta & \text { deletion/truncation } \\ & \end{array}$




\section{AMINO ACIDS AND DEOXYRIBONUCLEOTIDES}

Table 0.1: Amino acids.

\begin{tabular}{l|l|l} 
NAME & 3-LETTER CODE & 1-LETTER CODE \\
\hline Alanine & Ala & A \\
Arginine & Arg & R \\
Asparagine & Asn & N \\
Aspartic Acid & Asp & D \\
Cysteine & Cys & C \\
Glutamic Acid & Glu & E \\
Glutamine & Gln & Q \\
Glycine & Gly & G \\
Histidine & His & H \\
Isoleucine & Ile & I \\
Leucine & Leu & L \\
Lysine & Lys & K \\
Methionine & Met & M \\
Phenylalanine & Phe & F \\
Proline & Pro & P \\
Serine & Ser & S \\
Threonine & Thr & T \\
Tryptophan & Trp & W \\
Tyrosine & Tyr & Y \\
Valine & Val & V \\
\hline
\end{tabular}

Table 0.2: Deoxyribonucleotides.

\begin{tabular}{l|l} 
NAME & CODE \\
\hline Deoxyadenisine monophosphat & A \\
Deoxycytidine monophosphat & C \\
Deoxyguanine monophosphat & G \\
Deoxythymidine monophosphat & T
\end{tabular}




\section{INTRODUCTION}

\subsection{The immune system}

Mammalian organisms are constantly exposed to pathogens of vast diversity. To defend themselves, mammals have developed a complex system that can be divided into the innate and the adaptive immune system. These two branches cooperate to provide efficient protection (Iwasaki and Medzhitov, 2015). The elements of the innate immune system represent the first defense that pathogens encounter when they penetrate a body. It involves anatomical barriers like the skin or the mucosa in the gut and the respiratory airways. Furthermore, specialized leukocytes such as macrophages and neutrophils recognize conserved patterns, which are often found on microorganisms, with their receptors leading to clearance of the pathogen mainly by phagocytosis (Janeway and Medzhitov, 2002).

The defense process that is initiated by the innate immune system starts fast after a pathogen invades the host but is not specialized enough to provide sufficient protection against changing characteristics, which pathogens develop and that challenge the host. Therefore, the adaptive immune system evolved. Here, lymphocytes, namely B and $T$ cells, recognize "foreign" antigens of big diversity with their receptors, which are characterized by their broad specificity due to somatic recombination. When the cells of the adaptive immune system recognize the antigen and additionally receive costimulatory signals, they are activated and develop into different types of effector cells. T cells activate other cell types and thereby constitute the cell-mediated immune response. In contrast, highly specific antibodies that are secreted by B cells constitute the humoral-immune response. These antibodies act through neutralization and opsonization of pathogens and activation of the complement system or effector cells leading to the elimination of pathogens in the end (Burton and Woof, 1992). Along with antibody secreting plasma cells also memory $B$ cells mature which provide quick and specific immunity in case of reinfections.

\subsection{The B cell antigen receptor}

Signaling of the B cell antigen receptor $(B C R)$ is a crucial step in B cell activation, proliferation and differentiation. The $\mathrm{BCR}$ is a multi-protein complex consisting of a membrane-bound immunoglobulin ( $\mathrm{mlg}$ ), which is responsible for detection of the antigen, and two transmembrane signaling subunits, Iga (CD79a) and Ig $\beta$ (CD79b) (Reth, 1992). The $\lg \alpha / \lg \beta$ heterodimer is non-covalently associated with the $\mathrm{mlg}$ and transduces signals via kinases into the cytoplasm (Campbell et al., 1991). The mlgs are composed of two $\mathrm{lg}$ 
heavy chains $(\mathrm{IgH})$ that are disulfide-linked and are each additionally covalently bound to an Ig light chain ( $\lg \mathrm{L})$. Variability of BCRs is generated by rearrangement of gene segments that code for heavy as well as light chains, respectively. This process is called somatic recombination (Schatz and Ji, 2011).

Encounter of an antigen leads to BCR signaling-dependent internalization of the BCR/antigen complex (Dykstra et al., 2001; Stoddart et al., 2002), which requires amongst others Casitas B-lineage lymphoma proto-oncogene (cbl) (Kitaura et al., 2007). This internalization process is directed by the partially redundant paths of actin cytoskeleton as well as lipid-rafts in association with or without clathrin-coated pits (Cheng et al., 1999; Brown and Song, 2001; Stoddart et al., 2002; Stoddart et al., 2005). Upon internalization, the antigen is processed and presented to cognate $T$ helper cells, which provide costimulatory signals to the B cell. Subsequently, the activated B cell can go different ways. It can either differentiate into a plasma cell for rapid antibody secretion or enter germinal centers where the BCR variability and affinity to cognate antigen is increased by somatic hypermutation. Thereby random mutations are introduced that lead to changes in the $\mathrm{N}$ terminal, variable parts of the $\lg \mathrm{H}$ and $\mathrm{Ig} \mathrm{L}$ chains, which interact with the antigen (Weigert et al., 1970). In addition, B cells can switch their IgH isotype in germinal centers, called class-switch recombination. Five different isotypes of immunoglobulins exist, which constitute different effector functions when secreted as antibodies. The $\lg \mathrm{H}$ classes expressed by naïve $B$ cells are $\mu$ and $\delta$. Upon activation of naïve $B$ cells, gene rearrangement results in expression of another $\lg \mathrm{H}$, namely $\mathrm{\gamma}, \varepsilon$ or $\alpha$. In this process, the $\mu$ and $\delta$ gene segments are deleted and one of the other constant heavy gene segments is expressed (Muramatsu et al., 2000). Importantly, only the effector part of the $\mathrm{lgH}$ changes, whereas the part determining antigen specificity is unaltered. The $\mu$ and $\delta$ chain cytoplasmic tails consist of only three amino acids, namely lysine $(K)$, valine $(V)$, lysine $(\mathrm{K})$. The $\mathrm{Y}$ and $\varepsilon$ cytoplasmic parts have next to KVK additional amino acids resulting in cytoplasmic domains, which contribute in addition to the $\lg \alpha / \lg \beta$ subunit to signal onset into the cell. In this context, signals transmitted by the BCR constitute a crucial role for the fate of a B cell, since imbalance in BCR signaling can cause either under- or overreacting immune responses.

\subsection{BCR signaling at a glance}

There are currently different opinions spread about how early BCR signaling events are initiated. The most supported one proposes that antigen binding mediates crosslinking of numerous BCRs that consequently cluster and translocate into lipid microdomains, called lipid rafts (Cheng et al., 1999). The second opinion concerning BCR initiation events 
proposes the BCRs to be oligomeric in resting cells and to dissociate into an active state upon antigen binding (Reth, 2001; Fiala et al., 2013). However, the exact mechanisms have to be elucidated (Engels et al., 2008). In both models, src family kinases, like Blk, Fyn or Lyn are enriched at microdomains in close proximity to antigen-bound BCRs and phosphorylate the heterodimer $\lg \alpha / \lg \beta$ at their cytoplasmic tails, which harbor one Immunoreceptor tyrosine-based activation motif, in short ITAM (Reth, 1989; Humphrey et al., 2005). In each ITAM, two tyrosines are present that upon phosphorylation serve as docking sites for proteins with Src-homology 2 (SH2) domains, like the spleen tyrosine kinase (Syk) (Rowley et al., 1995). Syk harbors two SH2 domains that are tandemly arranged and allow the protein to bind the two phosphorylated tyrosine residues of one ITAM. Therefore, at least two Syk kinases get in close proximity so that they can phosphorylate and thereby fully activate themselves (Rolli et al., 2002). In addition, Syk activation is enhanced by trans-phosphorylation of the src kinase Lyn. In its active state, Syk can interact with and phosphorylate the adaptor protein $\mathrm{SH} 2$ domain-containing leukocyte adaptor protein of $65 \mathrm{kDa}$ (SLP65) (Wienands et al., 1998) also known as B cell linker (BLNK) (Fu et al., 1998) or B cell adaptor containing an $\mathrm{SH} 2$ domain (BASH) (Goitsuka et al., 1998). Phosphorylated SLP65 assembles the so called calcium $\left(\mathrm{Ca}^{2+}\right)$ initiation complex consisting of Bruton's tyrosine kinase (Btk) and phospholipase C (PLC) Y2 next to SLP65 itself (Engelke et al., 2007) and mediates its translocation to the plasma membrane. At the plasma membrane, PLCY2 gets in contact with its substrate the phospholipid phosphatidyl-inositol-4,5-bisphosphate $\left(\mathrm{PIP}_{2}\right)$ and hydrolyses it into the two second messengers diacylglycerol (DAG) and inositol-1,4,5-trisphosphate $\left(\mathrm{IP}_{3}\right)$. These two second messengers are crucial in BCR-induced signaling leading to activation of several downstream pathways.

The soluble second messenger $\mathrm{IP}_{3}$ is the ligand for $\mathrm{IP}_{3}$ receptors, which constitute $\mathrm{Ca}^{2+}$ channels, located in the membrane of the endoplasmic reticulum (ER). Upon ligand binding, $\mathrm{Ca}^{2+}$ from the lumen of the $\mathrm{ER}$ can passively pass the channel along the concentration gradient. Depletion of $\mathrm{Ca}^{2+}$ levels in the ER lumen is sensed by the stromal interaction molecule 1 (STIM1) that is located in the membrane of the ER (Putney Jr., 1986; Soboloff et al., 2012). This causes clustering of STIM1 and its translocation to the plasma membrane where it activates the $\mathrm{Ca}^{2+}$ release-activated $\mathrm{Ca}^{2+}$-channel (CRAC) ORAI (Cahalan, 2009) by direct interaction. ORAl functions as a store-operated $\mathrm{Ca}^{2+}$ ion channel and its activation initiates $\mathrm{Ca}^{2+}$ influx form the extracellular space (Zhang et al., 2006). The consequence of $\mathrm{Ca}^{2+}$ mobilization is the activation of different transcription factors that determine the fate of the cell.

Collectively, rapid and sustained increase in cytosolic $\mathrm{Ca}^{2+}$ is the key activation step in $\mathrm{B}$ cells upon BCR engagement that then branches into different pathways (Engels et al., 
2008). Although a lot is known about the events and interactors in BCR signaling, this important process is not entirely understood. One of the unanswered questions is how SLP65 is recruited to the plasma membrane upon BCR stimulation to ensure translocation of the $\mathrm{Ca}^{2+}$ initiation complex and hence activation of further downstream signaling factors like Nuclear factor of $k$ light polypeptide gene enhancer in $B$ cells (NF-kB).

\subsection{SLP65 function depends on the support of its constitutive interactor CIN85}

SLP65 constitutes the key adaptor protein of the $\mathrm{Ca}^{2+}$ initiation complex, whose function is fundamental for B cells, since a patient with a nonsense mutation in the gene coding for SLP65 shows symptoms of a primary immunodeficiency. This is marked by a block in the pro B cell stage and consequently low circulating B cells and almost absent serum antibodies (agammaglobulinemia) (Minegishi et al., 1999).

SLP65 is a crucial platform in BCR signaling events as it gathers numerous BCR effector proteins and passes on the signals derived from the antigen/BCR complex. Upon SLP65mediated $\mathrm{Ca}^{2+}$ initiation complex formation, Syk or Lyn activate Btk, which in turn phosphorylates PLCY2. Besides SLP65-initiated plasma membrane recruitment, binding of Btk and PLCY2 by the virtue of their pleckstrin homology $(\mathrm{PH})$ domains to the membrane-resident phosphatidylinositol 3,4,5 trisphosphate $\left(\mathrm{PIP}_{3}\right)$ (Salim et al., 1996; Marshall et al., 2000) supports plasma membrane recruitment of the complex (Falasca et al., 1998; Varnai et al., 1999). For proper function of SLP65, its phosphorylation by Syk is not sufficient. Furthermore, SLP65 has to be recruited to the plasma membrane. Our group revealed that for full functionality of SLP65, interaction with the adaptor protein Cblinteracting protein of $85 \mathrm{kDa}$ (CIN85) is required (figure 1.1). CIN85 (human) (Take et al., 2000), also termed Ruk (regulator of ubiquitous kinase) (Gout et al., 2000) in rat, SETA (SH3 domain-containing gene expressed in tumorigenic astrocytes) (Borinstein et al., 2000) in rat and SH3KBP1 (SH3 domain kinase binding protein 1) (Narita et al., 2001) in mice, is a constitutive interactor of SLP65 and is shown to be a prerequisite not only for SLP65 plasma membrane recruitment, but also BCR-initiated phosphorylation of SLP65 and hence $\mathrm{Ca}^{2+}$ signaling in the chicken DT40 B cell line (Oellerich et al., 2011). Direct interaction between CIN85 and SLP65 contributes to SLP65 membrane targeting in the chicken DT40 B cells (Oellerich et al., 2011). However, it is still unknown how CIN85 fulfills this support of SLP65 in the process of plasma membrane recruitment as the membrane anchor for CIN85 is unidentified. 


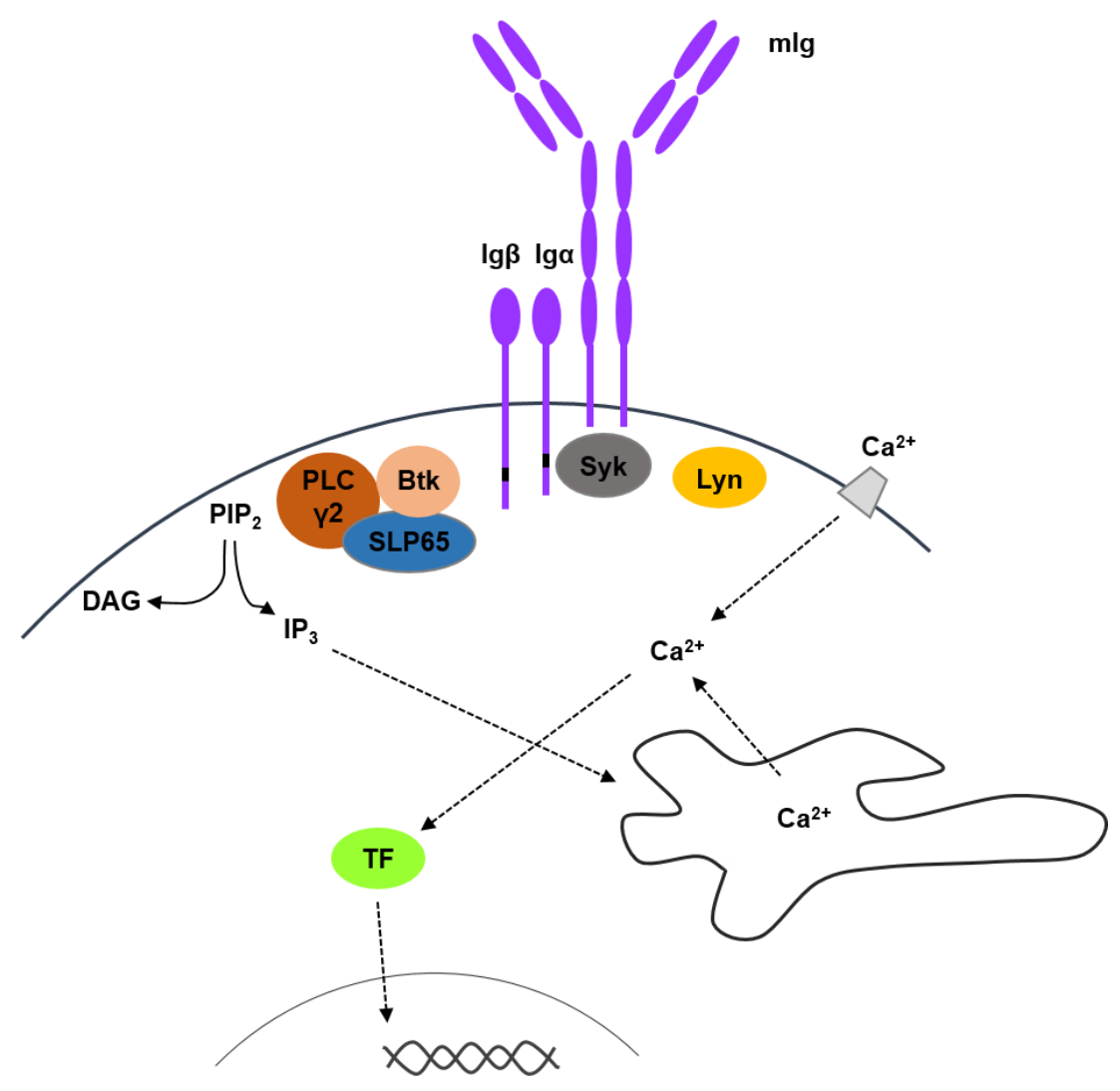

Figure 1.1: BCR-induced signal transduction leads to cytosolic enrichment of the second messenger $\mathrm{Ca}^{2+}$. Upon binding of the antigen, BCRs cluster and Lyn mediates phosphorylation of the ITAMs in the cytoplasmic tail of the $\lg \alpha / \lg \beta$ heterodimer. This initiates translocation and subsequent activation of Syk, which in turn phosphorylates SLP65 that is a steady ligand of CIN85. Phosphorylated SLP65 subsequently recruits Btk and PLCY2 to the plasma membrane. In this calcium initiation complex, Btk phosphorylates and activates PLCY2, which hydrolyzes $\mathrm{PIP}_{2}$ into the two second messengers $D A G$ and $\mathrm{IP}_{3} . \mathrm{IP}_{3}$ initiates $\mathrm{Ca}^{2+}$ influx from the ER with subsequent additional influx from the extracellular space. Enriched $\mathrm{Ca}^{2+}$ concentration in the cytosol leads to transcription factor (TF) activation and cell fate determination. Solid black line indicates hydrolysis, dotted black line indicates translocation.

SLP65 is exclusively expressed in B cells and macrophages and shares common structural features with the adaptor protein $\mathrm{SH} 2$ domain containing leucocyte protein of 76 $\mathrm{kDa}$ (SLP76), which in T cells transduces signals from the activated $\mathrm{T}$ cell receptor to the cytosol (Bonilla et al., 2000).

The domain architecture of SLP65 (figure 1.2) consists of an N-terminal region that is positively charged (Herrmann, 2009), followed by a region harboring several proline-rich motifs that can be bound by proteins expressing SH3 domains and tyrosine residues that are phosphorylated upon activation serving as interaction sites for $\mathrm{SH} 2$ domain containing proteins. The C-terminus of the protein contains an $\mathrm{SH} 2$ domain.

The overall structure of the interactor of SLP65, CIN85, is composed of three N-terminal $\mathrm{SH} 3$ domains, a proline-rich sequence and a coiled coil (CC) domain at its C-terminus 
(figure 1.2). Besides CIN85 also its family member CD2-associated protein (CD2AP) (Dustin et al., 1998) (also termed CMS (Cas ligand with multiple SH3 domains) (Kirsch et al., 1999) or METS-1 (mesenchyme-to-epithelium transition protein with SH3 domains) (Lehtonen et al., 2000)) is a steady ligand of SLP65 (Oellerich et al., 2011). Both CIN85 and CD2AP are ubiquitously expressed adaptor proteins that constitute their own CIN85/CD2AP family (Dikic, 2002) sharing $54 \%$ similarity in their amino acid sequence and display the same overall structure (figure 1.2).

The three SH3 domains of CIN85 and CD2AP enable interaction with proline-arginine motifs whereas the proline-rich regions in the central region of CIN85 provide docking sites for SH3 domain containing proteins themselves. The structure of the $\mathrm{CC}$ domain in the C-terminal region of CIN85 was recently solved (Kühn, 2015). It promotes either homo- or hetero-oligomerization with the CC of CD2AP (Kirsch et al., 1999; Borinstein et al., 2000; Watanabe et al., 2000). Moreover, another mechanism of CIN85 homodimer formation is mediated by binding of the first two $\mathrm{SH} 3$ domains to a proline rich region in the central region of CIN85 (Tibaldi, 2003). CD2AP additionally harbors four actin binding sites in its $\mathrm{C}$-terminal part that mediate interaction with the cytoskeleton and are missing in CIN85 (Kirsch et al., 1999; Welsch et al., 2005; Zhao et al., 2013).

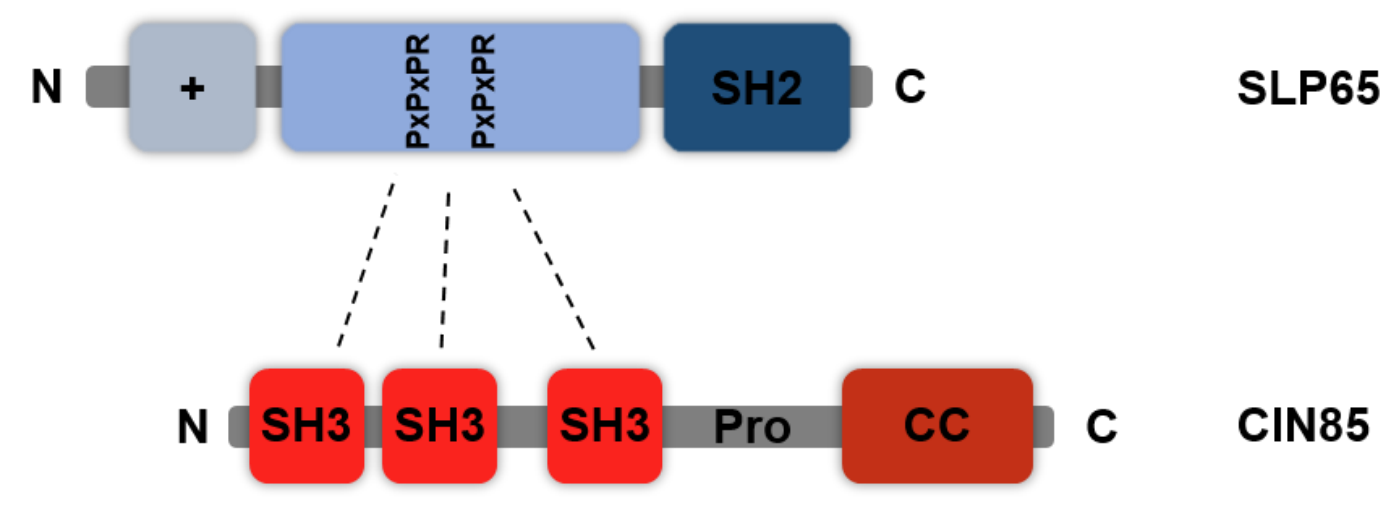

Figure 1.2: SLP65 and CIN85 domain architecture and interaction. The first 50 amino acids of SLP65 are composed of several positive charged amino acids $(+)$, followed by the central part containing tyrosine residues that are phosphorylated upon activation (not displayed) and a proline-rich region with the two motifs of the PxPxPR type, which mediate interaction with CIN85 (dotted lines). At its C-terminal part, SLP65 harbors a $\mathrm{SH} 2$ domain by which it can interact with phosphorylated tyrosine residues of proteins (Koretzky et al., 2006). In the N-terminal part of CIN85, three SH3 domains are present followed by a proline-rich region (Pro), which serves as interaction site for SH3 domains. At its C-terminus, CIN85 harbors a coiled coil domain (CC), which mediates homo- or hetero-oligomerization with CD2AP (Tibaldi, 2003).

The SH3 domains of CIN85 mediate interaction with atypical proline-arginine motifs in the central part of SLP65, which share the consensus sequence PxxxPR ( $P$ stands for 
proline, $\mathrm{x}$ for any amino acid and $\mathrm{R}$ for arginine) whereas PxPxPR is even better for binding of the CIN85 SH3 domain (Kowanetz et al. 2003). SLP65 harbors three of these motifs: PPSVPR ${ }_{49}$, PSPLPR $_{248}$ and PIPLPR PI13 $_{31}$ (Oellerich et al. 2011). CIN85 comprises three $\mathrm{SH} 3$ domains that can interact with all of the atypical proline-arginine motifs, whereas the first motif (49) is dispensable (figure 1.2). Since SLP65-CIN85 interaction is pivotal for efficient SLP65 membrane targeting, CIN85 constitutes an anchoring mode for SLP65. Moreover, the CIN85 family member CD2AP, which interacts with SLP65 by the same proline-arginine motif, can only partially compensate for the loss of CIN85 (Oellerich et al., 2011; Bremes, 2012). This highlights the particular need for the CIN85-SLP65 interaction in the context of BCR signaling.

It was recently found by our group that the N-terminal positively charged region of SLP65 interacts with lipids and mediates association with intracellular curved membranes. Therefore, the N-terminus of SLP65 contributes to SLP65 membrane targeting upon BCR activation that is essential for complete $\mathrm{Ca}^{2+}$ mobilization (Engelke et al., 2014). However, it was also shown that the N-terminus alone is not sufficient for SLP65 plasma membrane recruitment (Engelke et al., 2014).

For the SLP65 SH2 domain, direct interaction with the phosphorylated non-ITAM motif in the cytoplasmic tail of the BCR signaling subunit Iga is reported (Engels et al., 2001). However, direct SLP65-Iga interaction is not sufficient to target SLP65 to the plasma membrane as disruption of this interaction by substituting the non-ITAM sequence reveals only minor effects in $\mathrm{Ca}^{2+}$ mobilization (Kabak et al., 2002). Deletion of the SH2 domain resulted in decreased membrane translocation of SLP65 (Abudula et al., 2007), which indicates that another interaction partner in addition to CIN85 contributes for efficient plasma membrane recruitment. However, neither this putative interactor nor the mechanism of CIN85 support is completely clear.

\subsection{CIN85 in BCR-induced NF-kB signaling}

Besides the described role of CIN85 in BCR-induced $\mathrm{Ca}^{2+}$ signaling, CIN85 is also known to be involved in BCR-induced NF-KB signaling. Previous work of our group demonstrated that the above described interaction between the adaptor proteins CIN85 and SLP65 functions as an activatory modulator of NF-KB signaling upon BCR activation. Here, it was shown that NF-KB activation is diminished in SLP65-deficient chicken DT40 B cells reconstituted with a SLP65 variant that is incompetent in binding CIN85 (Oellerich et al., 2011). In addition, Kometani and colleagues could show that in primary CIN85-deficient mouse B cells, NF-kB activation upon BCR stimulation is reduced (Kometani et al., 2011). 
Recently, two male patients, who lack CIN85 expression, were identified by Dr. Moneef Shoukier from the human genetics department in Göttingen. These patients are siblings that have the same $247 \mathrm{kbp}$ deletion in their SH3KBP1 gene located on the $\mathrm{X}$ chromosome coding for CIN85. They both suffered from recurrent bacterial infections and consequently came into the clinics. It was diagnosed that their serum antibody titers were diminished, even though to different extents. In the case of the younger brother, $\lg M$ and $\lg _{4}$ levels were almost undetectable, whereas in the older brother, who died upon a septic shock, all immunoglobulin isotypes were reduced. In contrast to the diminished serum antibody levels, the peripheral B and T cell compartments of the younger brother equaled the reference values. Analysis of the responsiveness of the patients' $T$ cells showed full activation upon TCR/CD28 stimulation in several readout systems, although CIN85 is known to function in T cell maturation (Navarro et al., 2007) and activation (Hutchings et al., 2003; Tibaldi, 2003). This is in contrast to the patients' B cells, which showed an impaired $\mathrm{Ca}^{2+}$ mobilization and NF-KB activation upon BCR stimulation. In contrast, Phorbol myristate acetate (PMA), CD40 ligand and CpG oligodeoxynucleotides activated the patients' $B$ cells in the same manner as the $B$ cells of the healthy controls indicating that CIN85 functions specifically downstream of the BCR. However, the point as well as mode of interaction of CIN85 in the BCR-induced NF-KB pathway is unknown. As I elucidated the role of CIN85 in BCR-induced NF-KB signaling as part of my PhD project, I will outline the signaling pathway in more detail.

The second messenger DAG, which is generated together with $\mathrm{IP}_{3}$ by $\mathrm{PLC} \gamma 2$, initiates the activation of the canonical NF-KB pathway. DAG is membrane-bound and mediates recruitment and activation of protein-kinase C $\beta$ (PKC $\beta$ ) (Nishizuka, 1992). PKC $\beta$ is essential for BCR-mediated canonical NF-KB and MAPK pathway (Jiang et al., 1998; Saijo et al., 2002; Su et al., 2002). Activated PKC $\beta$ interacts with and phosphorylates the CARD-containing MAGUK protein 1 (CARMA1) (Sommer et al., 2005). Phosphorylated CARMA1 in turn forms together with the additional adaptor proteins Mucosa-associated lymphoid tissue lymphoma translocation protein 1 (MALT1) and B-cell lymphoma/leukemia 10 (Bcl10) a trimolecular complex known as CBM complex (Shinohara et al., 2007). Loss of each of these CBM complex proteins shows reduced B1 cell numbers and BCR-CD40 mediated impaired NF-KB activation and proliferation in mice (Ruland et al., 2001; Egawa et al., 2003; Hara et al., 2003; Xue et al., 2003). In more detail, upon activation of CARMA1, it homo-oligomerizes via its CC domain and Bcl10 together with MALT1 are recruited (Sommer et al., 2005; Tanner et al., 2007). The processes of oligomerization and formation of the CBM complex are tightly regulated by phosphorylation and ubiquitinylation (Egawa et al., 2003; Thome and Weil, 2007). 
CBM complex formation and activation induces recruitment of the Transforming growth factor beta-activated kinase (TAK) complex. This complex consists of TAK1 and one or more TAK1 binding proteins (TABs). TAK1 in turn recruits and subsequently activates another trimolecular complex, named IKB kinase (IKK) complex (Wang et al., 2001; Dai et al., 2012). The IKK complex is built out of the two catalytical subunits IKKa, IKK $\beta$ and the regulatory subunit IKKY (also termed NF-KB essential modulator (NEMO)). As the IKK complex is described to have a high molecular weight of $700-900 \mathrm{kDa}$ it is likely that two dimers of IKKa with IKK $\beta$ are hold together by IKKY (Karin and Ben-Neriah, 2000). However, the exact composition is not clear until now and involvement of other proteins is conceivable.

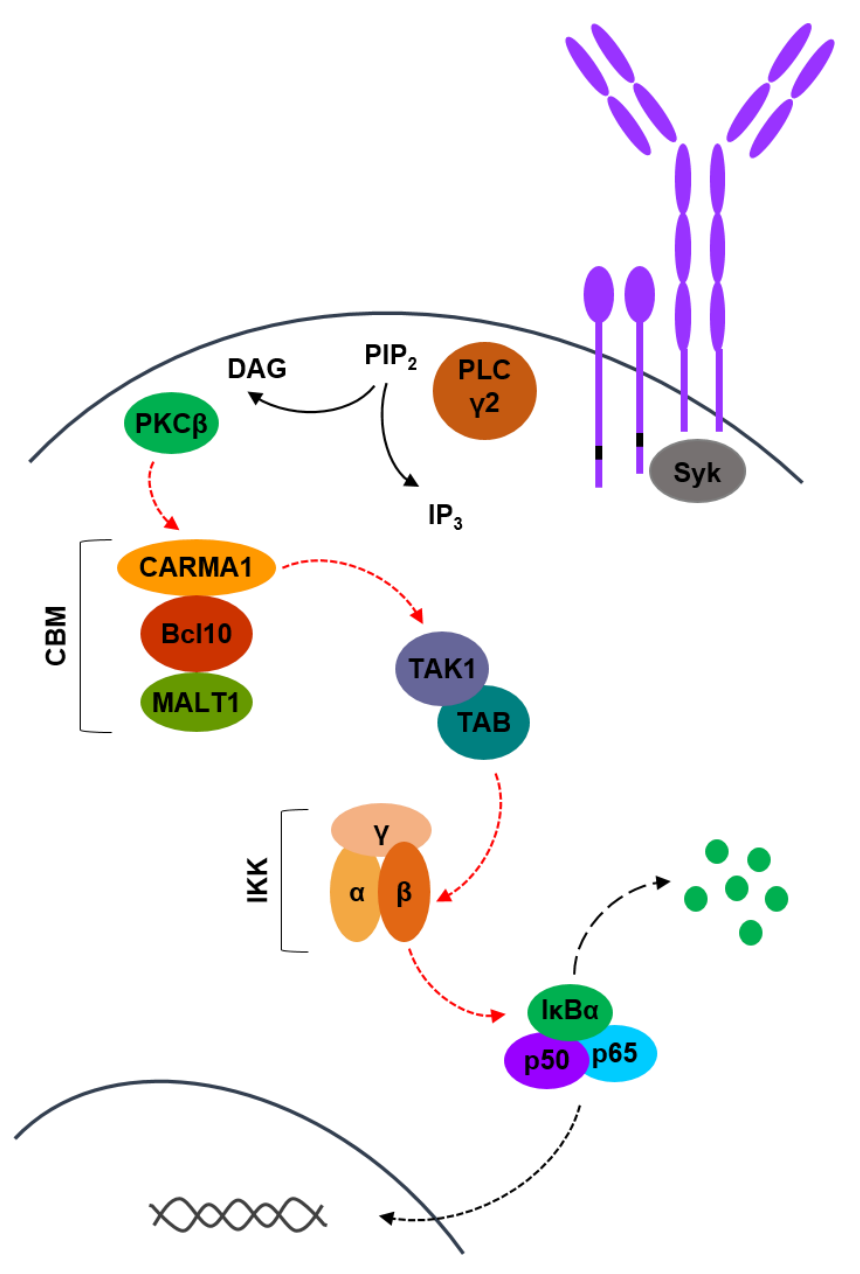

Figure 1.3: BCR-mediated signaling in the context of NF-kB activation. Upon BCR stimulation, PLCY2 is activated and hydrolyzes $\mathrm{PIP}_{2}$ into $\mathrm{DAG}$ and $\mathrm{IP}_{3}$. Subsequently, $\mathrm{Ca}^{2+}$ concentration is increased, which together with DAG activate PKC $\beta$. This in turn recruits and activates the CBM complex (CARMA1, Bcl10, MALT1), which phosphorylates TAK1 in a complex with TAB proteins. Thereupon, activated TAK1 activates the IKK complex (IKKa, $\beta$ and $\gamma$ ). IKK $\beta$ then phosphorylates IKBa, which is marked for degradation and in turn releases the NF-KB heterodimer p50/p65 into the nucleus where it can initiate transcriptional processes. Solid black line indicates hydrolysis, dotted black line indicates translocation, dotted red line indicates phosphorylation and dashed black line indicates degradation. 
IKKY couples the catalytical subunits, especially IKK , to the upstream signaling components and thereby mediates their phosphorylation by TAK1 or transautophosphorylation on several serine residues. These phosphorylations within IKKa/IKK $\beta$ as well as ubiquitin-induced oligomerization regulate their activation and bring them in close proximity. Ubiquitinylation of IKK does not mark in this particular case the protein for degradation but rather mediates its activation (Chen et al., 1996; Deng et al., 2000). Additionally, the two regulatory subunits of the TAK1 and IKK complex, namely TAB2 (Kanayama et al., 2004) and IKKy (Wu et al., 2006), respectively, are capable of interacting with ubiquitin, leading to TAK1 and IKK activation. The next step in the signaling cascade is the phosphorylation of $1 \mathrm{KB \alpha}$ by $\mathrm{IKK} \beta$, which marks $1 \mathrm{~KB} \alpha$ for proteasomal degradation. In resting cells, IKBa binds to NF-KB subunit dimers and thereby masks their nuclear localization signals (NLSs). Upon degradation of IKBs, the NF-KB subunits can translocate into the nucleus and activate gene transcription (Karin, 1999) (figure 1.3).

In contrast to the described canonical NF-KB pathway, the noncanonical pathway is activated by lymphotoxin $\beta$ (Xiao et al. 2001), B-cell-activating factor or CD40 ligand (Coope et al. 2002). It is classified by activation of specific NF-kB subunits but does very likely not play a role upon BCR stimulation and is not further analyzed in this PhD project. The NF-KB family of transcription factors is involved in the regulation of innate and adaptive immune responses, inflammation, cellular stress responses, apoptosis, cell growth and differentiation. Because of this broad spectrum of outcomes, the function of $\mathrm{NF}-\mathrm{kB}$ has to be tightly regulated to prevent dysregulation and thereby human diseases (Oeckinghaus et al., 2011).

\subsection{CIN85 orchestrates signaling pathways downstream of several receptors}

CIN85 is an adaptor protein that interacts with a large number of proteins (Dikic, 2002; Havrylov et al., 2009; Büchse et al., 2011). As such it is involved in the assembly as well as targeting of protein complexes to sub-cellular areas, which is necessary to transmit signals to downstream effectors (Wu and Koretzky, 2004; Cantrell, 2015). Formation of multimeric complexes allows for cooperation of otherwise separated pathways whereby a more complex network for fulfilling biological functions is generated.

As a networking platform, CIN85 is involved in T cell activation (Dustin et al., 1998; Tibaldi, 2003), receptor endocytosis and receptor tyrosine kinase signaling (Petrelli et al., 2002; Kobayashi et al., 2004). Nevertheless, little is known about its function in B cells. 
CD2AP was found as an associated protein of the $\mathrm{T}$ cell transmembrane protein cluster of differentiation 2 (CD2) in T cells (Dustin et al., 1998). CD2 harbors a proline-arginine sequence in its cytoplasmic part, which serves as site of interaction for the CD2AP and CIN85 SH3 domains (Dustin et al., 1998; Borinstein et al., 2000). CD2AP was described to mediate clustering of CD2 receptors and formation of an "immunological synapse" (Dustin et al., 1998). It is thought that CIN85 and CD2AP have redundant roles in terms of CD2 (Borinstein et al., 2000), which is supported by high similarity between both their three SH3 domains (Dikic, 2002). Additionally, the proline-rich regions of CIN85 as well as CD2AP serve as sites of interaction for the $\mathrm{SH} 3$ domains of several src kinases in vitro (Kirsch et al., 2001; Narita et al., 2005).

Further reports describe CIN85 involvement in epidermal growth factor receptor (EGFR) signaling. The inducible interactor of CIN85, the E3 ubiquitin-protein ligase cbl, recruits CIN85 upon ligand-mediated EGFR activation to the site of the receptor where the complex out of CIN85 and cbl controls receptor internalization (Soubeyran et al., 2002; Kobayashi et al., 2004). This influence on receptor degradation is on top also shown for other receptor tyrosine kinases than the EGFR (Petrelli et al., 2002; Szymkiewicz et al., 2002). In contrast to these findings other publications postulate that CIN85 is not involved in endocytosis of the EGFR but rather in the regulation of specific membrane trafficking processes (Havrylov et al., 2008; Havrylov et al., 2010; Ahmad et al., 2014), which is regulated by serine and threonine phosphorylations in CIN85 (Havrylov et al., 2010). In addition, it was reported that CIN85 interaction with the cbl isoform c-cbl leads to monoubiquitinylation of CIN85 at its C-terminus, which occurs upon EGFR activation and influences internalization and intracellular sorting of the polyubiquitinylated EGFR (Haglund et al., 2002). Monoubiquitinylation is defined as the attachment of a single ubiquitin on a lysine residue (Hicke and Dunn, 2003) whereas polyubiquitinylation is the modification by multiple ubiquitins that are linked to each other (Hershko and Heller, 1985). Noteworthy, polyubiquitinylation chains can be linked to each other in different ways leading to different signaling outcomes. One type of ubiquitin modification marks proteins for proteasomal degradation (Hershko and Ciechanover, 1998) whereas others fulfill regulatory tasks independent of degradation (Haglund et al., 2003; Krappmann and Scheidereit, 2005), like in the case of CIN85. Next to CIN85 ubiquitinylation, its SH3 domains possess ubiquitin binding capability (Stamenova et al., 2007; Bezsonova et al., 2008). Ubiquitin is thought to compete with other SH3 domain interaction partners for CIN85 binding (Bezsonova et al., 2008). In addition, CIN85 is shown to positively influence c-cbl mediated ubiquitinylation and degradation of the stimulated FcyRlla receptor in a PKC dependent manner in neutrophils (Marois et al., 2011). 
The two cbl isoforms that interact with CIN85, c-cbl and cbl-b (Take et al., 2000; Havrylov et al., 2009), are substrates of protein tyrosine kinases (Home et al., 2005; Song et al., 2010). Following activation of numerous receptors they function as negative regulators by interacting with receptor or nonreceptor tyrosine kinases (Thien and Langdon, 2001) like Src (Yokouchi et al., 2001; Kim et al., 2004) and Syk (Ota and Samelson, 1997; Rao et al., 2001).

An additionally reported interaction partner of CIN85 is the $\mathrm{SH} 2$ domain-containing inositol phosphatase 1 (SHIP-1) that is thought to downregulate $\mathrm{PIP}_{3}$ levels in $\mathrm{B}$ cells (Damen et al., 1995) and thereby terminate BCR responses. This report supports CIN85 meaning as a negative regulator in receptor signaling (Büchse et al., 2011). Also overexpression of CIN85 in B cells was reported to inhibit Syk and PLCY2 phosphorylation as well as BCRinduced $\mathrm{Ca}^{2+}$ flux (Niiro et al., 2012), which is in contrast to the mentioned results of our group in the chicken DT40 B cell line, where loss of interaction of SLP65 with CIN85 as well as knock down of CIN85 led to diminished BCR-induced $\mathrm{Ca}^{2+}$ mobilization (Oellerich et al., 2011; Bremes, 2012).

Taken together the bulk of reports about CIN85 concern its impact on different signaling pathways, regulating a diverse set of mechanisms like internalization, ubiquitinylation or membrane trafficking. However, most of the things known about CIN85 are derived from its interactors but its specific role remains elusive. Especially in B cells, little is known about the exact role of CIN85.

\subsection{Aim of this project}

The adaptor protein CIN85 has been shown to be a crucial factor in BCR-induced signaling. It mediates SLP65 plasma membrane recruitment and thereby enhances $\mathrm{Ca}^{2+}$ mobilization in the chicken DT40 B cell line. Likewise an influence of CIN85 in BCRinduced NF-KB signaling was shown in CIN85-deficient mouse and patients B cells. However, primary cells are limited in genetical and biochemical approaches so that mechanistic investigations of CIN85 are rather difficult. The so far used chicken B cell line DT40 already revealed importance of CIN85 by performance of shRNA-mediated knock down of CIN85 resulting in residual protein expression. Generation of a CIN85-deficient B cell line was not of success up to now. To further elucidate the function of CIN85, I focused my work on the following aspects:

1) Generation of a human CIN85-deficient DG75 B cell sub-line with the TALEN gene targeting method to investigate BCR-induced signaling processes for better comparison with the human patient. 
2) General influence of the adaptor protein CIN85 in BCR-induced $\mathrm{Ca}^{2+}$ mobilization and plasma membrane recruitment in human B cells.

3) Ubiquitinylation as potential regulatory mechanism of CIN85 in BCR proximal signaling.

4) Elucidation of the CIN85 NF-kB axis upon BCR activation. 


\section{MATERIALS \& METHODS}

\subsection{Materials}

\subsubsection{Chemicals and reagents}

Chemicals and reagents were purchased from Invitrogen, Merck, Roth or Sigma if not otherwise stated in the respective chapter.

\subsubsection{Consumable goods}

Table 2.1: Consumables used in this project.

\begin{tabular}{|c|c|}
\hline CONSUMABLE GOOD & COMPANY \\
\hline 4-well imaging chambers & Lab Tek \\
\hline $6 \times$ DNA loading buffer & New England biolabs \\
\hline 96-well optical reaction plates & Applied Biosystems \\
\hline ABsolute Blue SYBR green & Thermo scientific \\
\hline Blotting paper whatman & GE Healthcare \\
\hline $\begin{array}{l}\text { Cell culture equipment (cryo tubes, culture } \\
\text { dishes, tubes, pipettes) }\end{array}$ & Greiner bio-one \\
\hline CHX PESTANAL & Sigma \\
\hline dNTPs & New England biolabs \\
\hline FCS & PAA \\
\hline Filtropur filters & Sarstedt \\
\hline GeneRuler 1kb DNA ladder & Fermentas \\
\hline Glutathione sepharose ${ }^{\circledR}$ 4Fast Flow & GE Healthcare \\
\hline Hexadimethrine Bromide (Polybrene) & Sigma \\
\hline Imaging dishes & Mobitek \\
\hline Immersion oil & Merck \\
\hline Indo-1 AM & Invitrogen \\
\hline IPTG (Isopropyl- $\beta$-D-thiogalactopyranosid) & Sigma \\
\hline MG132 & Calbiochem \\
\hline Microscope paper & neoLab \\
\hline Neubauer improved counting chamber & Brand \\
\hline Nitrocellulose membrane Hybond ECL & Amersham biosciences \\
\hline PCR tubes & Sarstedt \\
\hline Pipette tips & Greiner bio-one \\
\hline Pipettes and electronic pipet filler & Eppendorf \\
\hline
\end{tabular}


Prestained protein marker, Broad Range

Protease Inhibitor Cocktail (P2714)

Sterile filter

Streptavidin-sepharose High Performance

Trans $\mid \mathrm{T}^{\circledR}$-293 Transfection Reagent

Trypsin/EDTA (0.05\%)

Ubiquitin-agarose

X-gal (5-bromo-4-chloro-3-indolyl-beta-Dgalacto-pyranoside)
New England biolabs

Sigma

Sarstedt

GE Healthcare

Mirus

Gibco

Enzo

Roth

\subsubsection{Enzymes}

The enzymes were used according to the manufacturer's instructions.

Table 2.2: Enzymes used in this project.

\section{ENZYME}

Calf intestinal phosphatase

Phusion high fidelity polymerase

PLASMID-SAFE ${ }^{\text {TM }}$

Proteinase $\mathrm{K}$

T4 DNA ligase

Type II restriction endonucleases

\section{COMPANY}

New England biolabs

New England biolabs

Biozym

Promega

New England biolabs

New England biolabs

\subsubsection{Reaction systems (Kits)}

Table 2.3: Ready to use kits used in this project.

KIT

First strand cDNA synthesis Kit

Human B Cell Nucleofector ${ }^{\circledR}$ Kit

Invisorb ${ }^{\circledR}$ Spin Plasmid Mini Two

Pure Yield TM Plasmid Midiprep System

RNAeasy ${ }^{\circledR}$ Mini Kit

The Original TA cloning ${ }^{\circledR}$ Kit

Wizard $^{\circledR}$ SV Gel and PCR clean up Kit

\section{COMPANY}

Fermentas

Lonza

Invitek

Promega

Qiagen

Invitrogen

Promega 


\subsubsection{Synthetic DNA oligonucleotides}

Synthetic DNA oligonucleotides were purchased from Eurofins Genomics.

Table 2.4: Primer used in this project.

Primer used for cloning in this project:

\begin{tabular}{|c|c|c|}
\hline NAME & SEQUENCE $5^{\prime}->3^{\prime}$ & APPLICATION \\
\hline mLynSH3fw & $\begin{array}{l}\text { AAGGATCCACAAAAGATCCAGAGGAA } \\
\text { CAA }\end{array}$ & $\begin{array}{l}\text { Cloning of LynSH3 into } \\
\text { pGEX }\end{array}$ \\
\hline hLynSH3re & $\begin{array}{l}\text { AACTCGAGACTCTCTTCTGTTTCTAA } \\
\text { GGTGTT }\end{array}$ & $\begin{array}{l}\text { Cloning of LynSH3 into } \\
\text { pGEX }\end{array}$ \\
\hline hRTKN2BamHIfw & $\begin{array}{l}\text { TAATGGATCCCTGGAGGGGCCGAGC } \\
\text { CTG }\end{array}$ & $\begin{array}{l}\text { Cloning of RTKN2 into } \\
\text { pMSCVpuro C1 }\end{array}$ \\
\hline hRTKN2Xholre & $\begin{array}{l}\text { TAATCTCGAGCTATACTTGTGCCTGC } \\
\text { AGCCATGATC }\end{array}$ & $\begin{array}{l}\text { Cloning of RTKN2 into } \\
\text { pMSCVpuro } \mathrm{C} 1\end{array}$ \\
\hline hPKCßBamHIfw & $\begin{array}{l}\text { TAATGGATCCCTGGCTGACCCGGCT } \\
\text { GCG }\end{array}$ & $\begin{array}{l}\text { Cloning of PKC } \beta \text { II into } \\
\text { pMSCVpuro } C 1\end{array}$ \\
\hline hPKC $\beta X$ holre & $\begin{array}{l}\text { TAATCTCGAGCTACACATTAATGACA } \\
\text { AACTCTGGGTTAGTATAA }\end{array}$ & $\begin{array}{l}\text { Cloning of PKCßI/PKCßII } \\
\text { into pMSCVpuro } \\
\text { C1/pMSCVblast }\end{array}$ \\
\hline $\begin{array}{l}\text { hPKCßBamHI_AT } \\
\text { Gfw }\end{array}$ & $\begin{array}{l}\text { TAATGGATCCATGGCTGACCCGGCT } \\
\text { GCG }\end{array}$ & $\begin{array}{l}\text { Cloning of } P K C \beta I / P K C \beta I I \\
\text { into pMSCVblast }\end{array}$ \\
\hline $\begin{array}{l}\text { hCARMA1BamHI } \\
\text { ATGfw }\end{array}$ & TTAAAGGATCCATGCCAGGAGGA & $\begin{array}{l}\text { Cloning of hCARMA1 into } \\
\text { pCit }\end{array}$ \\
\hline $\begin{array}{l}\text { hCARMA1BamHIr } \\
\text { e }\end{array}$ & $\begin{array}{l}\text { TAATGGATCCAGCAGCTGGTCCTCGT } \\
\text { CCACCCAG }\end{array}$ & $\begin{array}{l}\text { Cloning of hCARMA1 into } \\
\text { pCit }\end{array}$ \\
\hline $\begin{array}{l}\text { hBcl10BmHI_AT } \\
\text { Gfw }\end{array}$ & $\begin{array}{l}\text { TTAAAGGATCCATGGAGCCCACCGCA } \\
\text { CCGTC }\end{array}$ & $\begin{array}{l}\text { Cloning of hBcl10 into } \\
\text { pCit }\end{array}$ \\
\hline hBcl10Agelre & $\begin{array}{l}\text { TAATACCGGTAGTTGTCGTGAAACAG } \\
\text { TACGTG }\end{array}$ & $\begin{array}{l}\text { Cloning of hBcl10 into } \\
\text { pCit }\end{array}$ \\
\hline hSLP65CTGBglllf & TAATAGATCTCTGGACAAGCTTAATAA & Cloning of SLP65M23 \\
\hline w & AATAACCGTCCCC & \\
\hline $\begin{array}{l}\text { hSLP65CTGXholr } \\
\text { e }\end{array}$ & $\begin{array}{l}\text { TAATCTCGAGTTATGAAACTTTAACTG } \\
\text { CATACTTCAGTCTGGTG }\end{array}$ & $\begin{array}{l}\text { Cloning of SLP65M23 } \\
\text { into pMSCVpuro C1 }\end{array}$ \\
\hline
\end{tabular}


Primer used for sequencing in this project:

\begin{tabular}{l} 
NAME \\
\hline EGFPC1fw \\
GEXfw \\
M13fw \\
M13re \\
MSCVfw \\
MSCVre
\end{tabular}

\begin{tabular}{|l} 
SEQUENCE 5' $^{\prime}>\mathbf{3}$ \\
GTCCTGCTGGAGTTCGTG \\
GGGCTGGCAAGCCACGTTTGGTG \\
TGTAAAACGACGGCCAGT \\
CAGGAAACAGCTATGACC \\
CCCTTGAACCTCCTCGTTCGACC \\
CAGACGTGCTACTTCCATTTGTC
\end{tabular}

Primer used for qRT-PCR in this project:

\begin{tabular}{l|l} 
TARGET GENE & SEQUENCE $5^{\prime}->\mathbf{3}^{\prime}$ \\
\hline SLP65fw & TTCAGAACAGGAAGCTGGCG \\
SLP65re & GGTTGTTTGGAATCATGGCCA \\
GAPDHfw & CACCATCTTCCAGGAGCGAG \\
GAPDHre & AGAGGGGGCAGAGATGATGA
\end{tabular}

Primer used for shRNA in this project:

\begin{tabular}{|c|c|c|}
\hline NAME & SEQUENCE $5^{\prime}->3^{\prime}$ & APPLICATION \\
\hline chRTKN2s & TGCTGTTGACAGTGAGCGCAGACAGAGTCTGAA & Cloning of anti- \\
\hline hRNA1 & $\begin{array}{l}\text { ACCAAACTAGTGAAGCCACAGATGTAGTTTGGTT } \\
\text { TCAGACTCTGTCTTTGCCTACTGCCTCGGA }\end{array}$ & $\begin{array}{l}\text { chicken RTKN2 } \\
\text { shRNA construct } 1\end{array}$ \\
\hline chRTKN2s & TGCTGTTGACAGTGAGCGCAGACAGAGTCTGAA & Cloning of anti- \\
\hline hRNA2 & $\begin{array}{l}\text { ACCAAACTAGTGAAGCCACAGATGTAGTTTGGTT } \\
\text { TCAGACTCTGTCTTTGCCTACTGCCTCGGA }\end{array}$ & $\begin{array}{l}\text { chicken RTKN2 } \\
\text { shRNA construct } 2\end{array}$ \\
\hline $\begin{array}{l}\text { hRTKN2 } \\
\text { shRNA1 }\end{array}$ & $\begin{array}{l}\text { TGCTGTTGACAGTGAGCGAACCCATAATCTGTCT } \\
\text { ATTAATTAGTGAAGCCACAGATGTAATTAATAGA } \\
\text { CAGATTATGGGTCTGCCTACTGCCTCGGA }\end{array}$ & $\begin{array}{l}\text { Cloning of anti- } \\
\text { human RTKN2 } \\
\text { shRNA construct } 1\end{array}$ \\
\hline
\end{tabular}

Primer used for TALEN in this project:

\begin{tabular}{|c|c|c|}
\hline NAME & SEQUENCE $5^{\prime}->3^{\prime}$ & APPLICATION \\
\hline CIN85Exon4fw & GCATTGCTCTGCTTTCTACTGCCTCT & $\begin{array}{l}\text { Activity test and } \\
\text { sequencing }\end{array}$ \\
\hline CIN85Exon4re & TCCTCTGGGGAAAGCCATTTTCTC & $\begin{array}{l}\text { Activity test and } \\
\text { sequencing }\end{array}$ \\
\hline SLP65Exon6fw & GTCAATAAGCAGTTGAAATTTTGGGCCT & Activity test \\
\hline SLP65Exon6re & GGAGGGGATAATATGAGGGGCACA & Activity test \\
\hline pCR8_F1 & TTGATGCCTGGCAGTTCCCT & Sequencing \\
\hline
\end{tabular}




\begin{tabular}{l|l|l} 
pCR8_R1 & CGAACCGAACAGGCTTATGT & Sequencing \\
TAL_R2 & GGCGACGAGGTGGTCGTTGG & Sequencing \\
SeqTALEN_5-1 & CATCGCGCAATGCACTGAC & Sequencing
\end{tabular}

Primer used for mutagenesis in this project:

\begin{tabular}{|c|c|}
\hline NAME & SEQUENCE $5^{\prime}->3^{\prime}$ \\
\hline hPKCbetaR99Af & AAGGGTCCAGCCTCCGATGACCCCGCCAGCAAACACAAGTTTAA \\
\hline w & GATCCA \\
\hline hPKCbeataR99A & TGGATCTTAAACTTGTGTTTGCTGGCGGGGTCATCGGAGGCTGG \\
\hline re & ACCCTT \\
\hline hPKCbetaS100A & AAGGGTCCAGCCTCCGATGACCCCCGCGCCAAACACAAGTTTAA \\
\hline fw & GATCCA \\
\hline hPKCbetaS100A & TGGATCTTAAACTTGTGTTTGGCGCGGGGGTCATCGGAGGCTGG \\
\hline re & ACCCTT \\
\hline $\begin{array}{l}\text { hRTKN2R560Af } \\
\text { w }\end{array}$ & AGAAACCAATGGCTGCTCCTGCAAAACTTCTGCCTGCCAGG \\
\hline $\begin{array}{l}\text { hRTKN2R560Ar } \\
\text { e }\end{array}$ & CCTGGCAGGCAGAAGTTTTGCAGGAGCAGCCATTGGTTTCT \\
\hline $\begin{array}{l}\text { hRTKN2R591Af } \\
\text { w }\end{array}$ & CCAAGCCAGTGCCAGCTCCAGCGCAGAAATCCATCAAAGACA \\
\hline $\begin{array}{l}\text { hRTKN2R591Ar } \\
\text { e }\end{array}$ & TGTCTTTGATGGATTTCTGCGCTGGAGCTGGCACTGGCTTGG \\
\hline my2afw & AGATCTAGATCTGAGCACACAGGACCTCACC \\
\hline my2aKVRre & $\begin{array}{l}\text { TATAGAATTCGCCCTTGAAGATTCACCGTACCTTGAAGAGTGTGA } \\
\text { CAG }\end{array}$ \\
\hline my2aRVKre & $\begin{array}{l}\text { TATAGAATTCGCCCTTGAAGATTCACTTTACCCGGAAGAGTGTGA } \\
\text { CAG }\end{array}$ \\
\hline my2aRVRre & $\begin{array}{l}\text { TATAGAATTCGCCCTTGAAGATTCACCGTACCCGGAAGAGTGTGA } \\
\text { CAG }\end{array}$ \\
\hline
\end{tabular}




\subsubsection{Plasmids}

Table 2.5: Plasmids used for cloning and expression in this project.

cDNA of Bcl10 and RTKN2 was amplified from self-generated DG75 cDNA. Vectors and cDNA templates used in this project:

\begin{tabular}{l|l} 
NAME & SOURCE/COMPANY \\
\hline pCR2.1 & Invitrogen \\
pGEX4T1 & GE Healthcare \\
pMSCV puro & BD Biosciences Clontech \\
pHCMV-VSV-G & M. Jücker \\
LMP-pMSCV-GFP & OpenBiosystems \\
pMSCVblast & M. Engelke \\
pmaxKS IRES-EGFP & N. Engels \\
pmaxKS IRES-tagRFP & C. Hitzing \\
pCitrine & M. Engelke \\
pCR2.1 Y2aKVK & N. Engels \\
pCR2.1 hPKC $\beta I$ & N. Engels \\
pCR2.1 hPKCßII & N. Engels \\
pMSCVpuro Citrine (C1) & V. Bremes \\
pCit SLP65 M23 & J. Kühn \\
pCR2.1 hSLP65wt & V. Bremes \\
pMSCV blast GFP & V. Bremes \\
pABESpuro mLyn_wt-HA & M. Lösing \\
pABESpuro mLyn_P114L-HA & M. Lösing \\
pCR2.1 hCARMA1 & H. Bohnenberger \\
\hline
\end{tabular}

Constructs for expression:

\begin{tabular}{l|l|l} 
NAME & INSERT & SOURCE \\
\hline pMSCVpuro & EGFP & C. Hitzing \\
pMSCVpuro & Cit CIN85 & V. Bremes \\
pMSCVpuro & Cit CIN85 $\triangle \mathrm{SH}_{3}$ & V. Bremes \\
pMSCVpuro & Cit CIN85 $\triangle \mathrm{CC}$ & V. Bremes \\
pMSCVpuro & Cit SLP65 M23 & This work \\
pMSCVpuro & Cit SLP65 & V. Bremes \\
pMSCVblast & GFP SLP65 & This work \\
pABESpuro & RFP CIN85 & V. Bremes \\
pMSCVpuro & Cit CD2AP & V. Bremes \\
pMSCVpuro & my2a KVK & N. Engels
\end{tabular}




\begin{tabular}{|c|c|c|}
\hline pMSCVblast & my2a KVK & This work \\
\hline pMSCVpuro & my2a KVR & This work \\
\hline pMSCVpuro & my2a RVK & This work \\
\hline pMSCVpuro & my2a RVR & This work \\
\hline pMSCVblast & my2a RVR & This work \\
\hline pcDNA3.1 & HA-Ubiquitin (8x) & N. Engels \\
\hline pMSCVblast & PKC $\beta I$ & This work \\
\hline pMSCVblast & PKC $\beta I I$ & This work \\
\hline pMSCVpuro & Cit PKCßII & This work \\
\hline pMSCVblast & 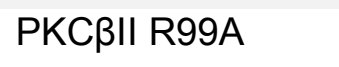 & This work \\
\hline pMSCVblast & PKC $\beta I I$ S100A & This work \\
\hline pMSCVpuro & Cit CARMA1 & This work \\
\hline pMSCVpuro & Cit Bcl10 & This work \\
\hline pMSCVpuro & Cit RTKN2 & This work \\
\hline pMSCVpuro & Cit RTKN2 R560A & This work \\
\hline pMSCVpuro & Cit RTKN2 R591A & This work \\
\hline
\end{tabular}

Expression of GST-fusion proteins:

\begin{tabular}{l|l|l} 
NAME & INSERT & SOURCE \\
\hline pGEX-6P1 & CIN85 & V. Bremes \\
pGEX-4T1 & CIN85 $\mathrm{SH}_{3}$ & V. Bremes \\
pGEX-4T1 & CIN85 PRC & V. Bremes \\
pGEX-4T1 & CIN85 SH3 A & V. Bremes \\
pGEX-4T1 & CIN85 SH3 B & V. Bremes \\
pGEX-4T1 & CIN85 SH3 C & V. Bremes \\
pGEX-4T1 & Syk SH2 & M. Engelke \\
pGEX-4T1 & Lyn SH3 & This work \\
pGEX-4T1 & Lyn SH3 P114L & This work
\end{tabular}

Expression of shRNA constructs:

\begin{tabular}{l|l|l} 
NAME & INSERT & SOURCE \\
\hline LMP-pMSCV-GFP & sh mock & V. Bremes \\
LMP-pMSCV-GFP & sh chCIN85_2389 & V. Bremes \\
LMP-pMSCV-GFP & sh hCD2AP_381 & V. Bremes \\
LMP-pMSCV-GFP & sh chRTKN21 & This work \\
LMP-pMSCV-GFP & sh chRTKN2 2 & This work \\
LMP-pMSCV-GFP & sh hRTKN21 & This work
\end{tabular}


TALEN cloning vectors:

\begin{tabular}{l|l} 
NAME & SOURCE \\
\hline pTal4Titanium & C. Hitzing \\
pFusA5A & C. Hitzing \\
pFusA5B & C. Hitzing \\
pFusA5C & C. Hitzing \\
pFusB1B & C. Hitzing \\
pFusB2B & C. Hitzing \\
pFusB3B & C. Hitzing \\
pFusB4B & C. Hitzing
\end{tabular}

Expression of TALEN gene targeting constructs:

\begin{tabular}{l|l|l} 
NAME & INSERT & SOURCE \\
\hline pmaxKS IRES EGFP & hCIN85 62,2 left & This work \\
pmaxKS IRES tagRFP & hCIN85 62,2 right & This work \\
pmaxKS IRES EGFP & hSLP65 39 & C. Hitzing \\
pmaxKS IRES tagRFP & hSLP65 55 & C. Hitzing
\end{tabular}

\subsubsection{Software}

Table 2.6: Software used in this project.

SOFTWARE
BD FACSDiva Software v 5.0.3.
Intas Chemostar
FlowJo (TriStar)
Gel documentation software GDS
ImageJ
Leica Confocal Software
MS Office
pDRAW 32
Citavi 4
FinchTV
7500 system SDS Software version
1.4.0.25
Ubiquitin site prediction software from the
Rockefeller University

\section{APPLICATION}

Flow cytometry data recording

Immunoblot imaging

Flow cytometry data analysis

Gel imaging

Image editing

Imaging

Text and Image editing

DNA sequence analysis

Bibliography editing

Sequencing viewer

qRT-PCR

Ubiquitin prediction site 


\subsubsection{Websites}

Table 2.7: Websites used in this project.

\begin{tabular}{l} 
WEBSITE \\
\hline http://expasy.org/ \\
http://multalin.toulouse.inra.fr/multalin/ \\
http://cancan.cshl.edu/rnai_central/rnai.cgi?type=sirna \\
http://www.ncbi.nlm.nih.gov/ \\
https://tale-nt.cac.cornell.edu/node/add/talen \\
http://www.ensembl.org \\
http://bioinfo.ut.ee/primer3-0.4.0/
\end{tabular}

\section{APPLICATION}

SIB Bioinformatics Resource

Portal

Multiple alignment

shRNA design

Genomic blast

TALEN design

Genomic blast

Primer3

\subsubsection{Antibodies}

Table 2.8: Antibodies used in this project.

Antibodies used for stimulation:

\begin{tabular}{|c|c|c|}
\hline ANTIBODY & TYPE & COMPANY \\
\hline goat-a-human IgM & $F\left(a b^{\prime}\right)_{2}$ & SouthernBiotech \\
\hline goat- $\alpha-m o u s e \lg M+\lg G$ & $F\left(a b^{\prime}\right)_{2}$ & Jackson ImmunoResearch \\
\hline a-chicken IgM M4 & mouse IgM & Biozol \\
\hline goat-a-human IgM-BIOT & $F\left(a b^{\prime}\right)_{2}$ & SouthernBiotech \\
\hline goat-a-mouse lgG-BIOT & $F\left(a b^{\prime}\right)_{2}$ & SouthernBiotech \\
\hline
\end{tabular}

Primary antibodies for immunoblotting. The antibodies were mixed in TBS-T supplemented with $1 \% \mathrm{BSA}$ and $0.01 \% \mathrm{NaN}_{3}$ and used according to the manufacturer's instructions:

\begin{tabular}{|c|c|c|}
\hline PRIMARY ANTIBODY & ISOTYPE & SOURCE/COMPANY \\
\hline a-Actin & rabbit & Sigma-Aldrich $^{\circledast}$ \\
\hline$\alpha-B c l 10(\mathrm{C} 78 \mathrm{~F} 1)$ & rabbit & CST \\
\hline a-BLNK (SLP65) & mouse $\lg G_{2 a}$ & $\mathrm{BD}$ \\
\hline a-CARD11 (CARMA1) & rabbit & CST \\
\hline$\alpha-c-c b l$ & mouse $\lg G_{1}$ & $\mathrm{BD}$ \\
\hline a-CD2AP (chicken) & rabbit & V. Bremes \\
\hline a-CD2AP (H-290) (human) & rabbit & Santa Cruz \\
\hline$\alpha-c h l g M$ & goat lgG & Bethyl Laboratories, Inc. \\
\hline a-CIN85 (C6115) (chicken) & rabbit & Sigma-Aldrich ${ }^{\circledR}$ \\
\hline
\end{tabular}




\begin{tabular}{|c|c|c|}
\hline a-CIN85 (D1A4) (human) & rabbit & CST \\
\hline a-GFP & mouse $\lg _{1} \mathrm{~K}$ & Roche \\
\hline$\alpha-G S T$ & rabbit & Molecular Probes \\
\hline a-lgalpha & rabbit & Abcam \\
\hline$\alpha-I K K \alpha$ & rabbit & CST \\
\hline$\alpha-I_{k B \alpha}$ & mouse $\lg \mathrm{G}_{1}$ & CST \\
\hline a-pAkt (Ser473) (D9E) & rabbit & CST \\
\hline$\alpha-p E r k 1 / 2(E 10)$ & mouse $\lg \mathrm{G}_{1}$ & CST \\
\hline $\begin{array}{l}\alpha-p l K K \alpha / \beta(\operatorname{Ser} 176 / 180) \\
(16 A 6)\end{array}$ & rabbit & CST \\
\hline a-plkBa (Ser32) (14D4) & rabbit & CST \\
\hline$\alpha-P K C \beta I$ & rabbit & Santa Cruz \\
\hline$\alpha-P K C \beta I I$ & mouse $\lg \mathrm{G}_{1}$ & Santa Cruz \\
\hline a-pSLP65 (pTyr96) & rabbit & CST \\
\hline a-pSyk (Tyr323) & rabbit & CST \\
\hline$\alpha-p T y r(4 G 10)$ & mouse lg $G_{2 b}$ & Upstate \\
\hline a-RTKN2 & rabbit & Sigma-Aldrich $^{\circledR}$ \\
\hline a-Syk (4D10) & mouse $\lg G_{2 a}$ & Santa Cruz \\
\hline a-Ubiquitin $6 \mathrm{C} 1$ & mouse $\lg _{2 a}$ & Sigma-Aldrich $^{\circledR}$ \\
\hline
\end{tabular}

Secondary antibodies. Antibodies were used diluted 1:10000 in TBS-T:

\begin{tabular}{l|l|l} 
SECONDARY ANTIBODY & ISOTYPE & COMPANY \\
\hline a-goat IgG-HRPO & donkey & Jackson ImmunoResearch \\
a-HA-Peroxidase & rat $\operatorname{lgG}_{1}$ & Sigma-Aldrich $^{\circledR}$ \\
a-mouse $\operatorname{lgG}_{1}-\mathrm{HRPO}$ & goat & SouthernBiotech \\
a-mouse $\operatorname{lgG}_{2 \mathrm{a}}-\mathrm{HRPO}$ & goat & SouthernBiotech \\
a-mouse $\operatorname{lgG}_{2 \mathrm{~b}}-\mathrm{HRPO}$ & goat & SouthernBiotech \\
a-mouse IgG-HRPO & goat & SouthernBiotech \\
a-rabbit IgG-HRPO & goat & SouthernBiotech \\
a-rat IgG-HRPO & goat & Pierce
\end{tabular}

Antibodies for FACS:

\begin{tabular}{l|l|l} 
ANTIBODY & ISOTYPE & COMPANY \\
\hline Streptavidin-APC & ---- & BD \\
$\alpha$-human IgM-APC & mouse $\operatorname{lgG}_{1}$ & SouthernBiotech \\
$\alpha$-mouse $\operatorname{lgG}_{2 \mathrm{a}}$-CY5 & goat & SouthernBiotech
\end{tabular}




\subsubsection{Instruments}

Table 2.9: Instruments used in this project.

INSTRUMENT

7500 Real Time PCR System

Analytical balance MC1

Bacteria incubator Heraeus Kelvitron ${ }^{{ }^{\circledR}} \mathrm{t}$

Bio Photometer

Cell culture incubator HeraCell 150

Centrifuge 5415D

Centrifuge 5417R

Centrifuge Multifuge 3 S-R

Centrifuge PMC-060

Centrifuge RC 3B Plus

Chemi Lux Imager

Confocal Laser scanning microscope TCS SP2

Cytometer LSRII

Electrophoresis Power Supply

Electroporation cuvette (4 mm gap)

FACS Calibur

Freezer HERAfreeze

Freezer Platilab 340

Gel Electrophoresis system

Gel Imager

GenePulser $^{\circledR}$ |l electroporation system

Ice machine

Light microscope TELAVAL 31

Magnetic stirrer M21/1

Mastercycler epgradient

Mini-PROTEAN Tetra Electrophoresis

System

NanoDrop 2000 Spectrophotometer

Nucleofector ${ }^{\circledR}$ II

$\mathrm{pH}$ meter

Platform shaker

Rotator SB3

Semi-dry transfer unit TE 77

\section{COMPANY}

Applied Biosystems

Sartorius

Heraeus

Eppendorf

Heraeus

Eppendorf

Eppendorf

Heraeus

Tomy

Sorvall ${ }^{\circledR}$

Intas

Leica

BD

Amersham Biosciences

PeqLab

$\mathrm{BD}$

Heraeus

Angelantoni life science

Peqlab

Intas

Bio-Rad

Ziegra

Zeiss

Framo ${ }^{\circledR}$-Gerätetechnik

Eppendorf

Bio-Rad

Thermo Scientific

Amaxa $^{\mathrm{TM}}$

inoLab $^{\circledR}$

GFL

Stuart

GE Healthcare 


\begin{tabular}{l|l} 
Shaker 3006 & GFL \\
Shaker ST5 & CAT \\
Shaking Incubator Infors & Unitron \\
ThermoStat plus & Eppendorf \\
Ultra-low Temperature Freezer $\left(-150^{\circ} \mathrm{C}\right)$ & Panasonic \\
Ultrasonic device Sonoplus & Bandelin \\
UV illuminator & Intas \\
Vortex Genie 2 & Scientific Industries \\
Water Purification System Milli-Q & Sartorius \\
Waterbath & Schütt Labortechnik
\end{tabular}

\subsection{Methods}

\subsubsection{Molecular Biology}

\subsubsection{Media for bacteria}

The bacterial E.coli strains were grown in LB-medium and for protein expression the E.coli were cultivated in YT-medium.

LB-medium: $10 \mathrm{~g} / \mathrm{l}$ tryptone

$5 \mathrm{~g} / \mathrm{l}$ yeast extract

$5 \mathrm{~g} / \mathrm{l} \mathrm{NaCl}$

$\mathrm{pH} 7.0$
YT-medium: $16 \mathrm{~g} / \mathrm{l}$ tryptone

$15 \mathrm{~g} / \mathrm{l}$ yeast extract

$5 \mathrm{~g} / \mathrm{l} \mathrm{NaCl}$

$\mathrm{pH} 7.0$

Both media were autoclaved $\left(121^{\circ} \mathrm{C}, 1.25 \mathrm{bar}, 30 \mathrm{~min}\right)$ before usage.

For selection the media were supplemented with antibiotics with the following end concentrations:

$\begin{array}{ll}\text { Ampicillin } & 100 \mu \mathrm{g} / \mathrm{ml} \\ \text { Kanamycin } & 50 \mu \mathrm{g} / \mathrm{ml} \\ \text { Spectinomycin } & 50 \mu \mathrm{g} / \mathrm{ml} \\ \text { Tetracyclin } & 10 \mu \mathrm{g} / \mathrm{ml}\end{array}$

\subsubsection{Agar plates}

The E.coli strains were spread on agar plates containing LB-medium with $2 \%(\mathrm{w} / \mathrm{v})$ agaragar. After autoclaving of the medium $\left(121^{\circ} \mathrm{C}, 1.25 \mathrm{bar}, 30 \mathrm{~min}\right)$ the medium was cooled down to $60{ }^{\circ} \mathrm{C}$ before the appropriate antibiotic was added and the LB-agar was poured into petri dishes. The plates were stored at $4{ }^{\circ} \mathrm{C}$ in the dark. 


\subsubsection{Sterilization}

Autoclaving of culture media for bacteria, solutions and non-sterile consumables was done at $121^{\circ} \mathrm{C}$ and 1.25 bar for $30 \mathrm{~min}$.

\subsubsection{E.coli strains}

During my $\mathrm{PhD}$ project, the E.coli strain Top10F' was used for all cloning experiments whereas the E.coli strain BL21 was used for protein expression.

Table 2.10: E.coli strains used in this project.

\begin{tabular}{|c|c|}
\hline E.COLISTRAIN & GENOTYPE \\
\hline Top10F' & $\begin{array}{l}\left.\text { F'}^{\prime} \text { laclq, Tn10(TetR) }\right\} \text { mcrA } \Delta \text { (mrr-hsdRMS-mcrBC) } \\
\text { Ф80lacZ } \Delta \text { M15 } \Delta \text { lacX74 recA1 araD139 } \Delta \text { (ara leu) } 7697 \text { galU galK } \\
\text { rpsL (StrR) endA1 nupG }\end{array}$ \\
\hline BL21 & $\begin{array}{l}\text { fhuA2 [lon] ompT gal }(\lambda \text { DE3) }[\mathrm{dcm}] \Delta \text { hsdS } \lambda \text { DE3 }=\lambda \text { sBamHlo } \\
\Delta \text { EcoRI-B int::(lacl::PlacUV5::T7 gene1) i2 } 1 \Delta \text { nin5 }\end{array}$ \\
\hline
\end{tabular}

\subsubsection{Isolation of genomic DNA}

$1 \times 10^{6}$ DG75 cells were resuspended in $100 \mu \mathrm{l}$ Tag-lysis buffer and incubated with $1 \mu \mathrm{l}$ proteinase $\mathrm{K}(20 \mathrm{mg} / \mathrm{ml})$ at $56{ }^{\circ} \mathrm{C}$ for $3 \mathrm{~h}$. The enzyme activity was heat inactivated at $95^{\circ} \mathrm{C}$ for 15 min and the DNA was stored at $-20^{\circ} \mathrm{C}$

Tag-lysis buffer: $\quad 10 \mathrm{mM}$ Tris/HCl, $\mathrm{pH} 8$

$50 \mathrm{mM} \mathrm{KCl}$

$0.45 \%$ NP40

$0.45 \%$ Tween20

in ${ }_{d d} \mathrm{H}_{2} \mathrm{O}$

\subsubsection{Isolation of $m R N A$}

$1 \times 10^{6}$ DG75 cells were used for mRNA preparation. Isolation was performed with the RNAeasy ${ }^{\circledR}$ mini kit from Qiagen according to the manufacturer's instructions.

\subsubsection{Isolation of plasmid DNA from E.coli}

$4 \mathrm{ml}$ LB medium supplemented with the appropriate antibiotics were inoculated with one

E.coli colony shaking overnight with $180 \mathrm{rpm}$ at $37^{\circ} \mathrm{C}$. The next day, bacterial plasmid DNA was isolated using the Invisorb ${ }^{\circledR}$ Spin Plasmid Mini Two Kit (Invitek) according to 
manufacturer's instructions. To gain higher amounts of plasmid DNA, $100 \mathrm{ml}$ of an overnight culture were used and the bacterial plasmid DNA was isolated using the Wizard $^{\circledR}$ Plus SV Midiprep Kit (Promega) according the manufacturer's instructions. The DNA was eluted in ${ }_{d d} \mathrm{H}_{2} \mathrm{O}$.

\subsubsection{DNA concentration determination}

Concentration of DNA was measured from $1 \mu \mathrm{l}$ using the NanoDrop2000 from Thermo scientific according to the manufacturer's protocol.

\subsubsection{Polymerase chain reaction (PCR)}

For in vitro amplification of DNA fragments (Mullis et al., 1986) as well as site directed mutagenesis, PCR reactions were performed with the phusion polymerase according to the manufacturer's instructions.

\subsection{Standard PCR}

The PCR reaction is subdivided into repetitive cycles, which comprise three repetitive steps. A cycle starts with denaturation of double-stranded DNA, followed by annealing of oligonucleotides (primers) and terminates with the elongation of the annealed primers. Primers were ordered at Eurofins Genomics, the annealing temperature of primers was calculated with Primer3 and the elongation time was set according to the length of the amplicon.

Table 2.11: Cycle conditions of a standard PCR.

\begin{tabular}{|c|c|c|c|}
\hline STEP & TIME & CYCLES & TEMPERATURE \\
\hline Initial denaturation & $2 \min$ & 1 & $98^{\circ} \mathrm{C}$ \\
\hline Denaturation & $20 \mathrm{sec}$ & 7 & $98^{\circ} \mathrm{C}$ \\
\hline Annealing & $40 \mathrm{sec}$ & 33 & according to Primer3 \\
\hline Elongation & $0.3-3 \min$ & J & $72^{\circ} \mathrm{C}$ \\
\hline Final elongation & $1-6 \min$ & 1 & $72^{\circ} \mathrm{C}$ \\
\hline
\end{tabular}

\subsection{Quantitative real time PCR}

Quantitative real time (qRT) PCR was performed to determine the relative amount of mRNA transcripts of a specific gene of interest. To measure the amount of doublestranded PCR products after each cycle the dye SYBR green, which intercalates in 
double-stranded DNA, was used. Therefore, cDNA from CIN85-deficient DG75 cells and CIN85-deficient DG75 cells expressing wild-type CIN85 were analyzed with primers for SLP65 and the housekeeping gene GAPDH. For each sample, $1 \mu \mathrm{L}$ of template cDNA was mixed with $1 \mu \mathrm{L}$ of a prediluted primer mix ( $5 \mathrm{pmol}), 10 \mu \mathrm{L}$ of the Blue SYBR Green PCR Master Mix and $9 \mu \mathrm{L}{ }_{d d} \mathrm{H}_{2} \mathrm{O}$. After each PCR cycle (see table 2.12), the amount of double-strand DNA was measured and calculated by the Applied Biosystems 7500 System SDS Software. Crossing threshold (CT) values of the target gene were substracted by the values of the housekeeping gene $(\Delta \mathrm{CT})$. The quantity was calculated as $2^{-\Delta \mathrm{CT}}$ relative to the reconstituted control.

Table 2.12: qRT-PCR protocol.

\begin{tabular}{l|l|l} 
STEP & TIME & TEMPERATURE \\
\hline Enzyme activation & $2 \mathrm{~min}$ & $50^{\circ} \mathrm{C}$ \\
Initial denaturation & $10 \mathrm{~min}$ & $95^{\circ} \mathrm{C}$ \\
Denaturation* & $15 \mathrm{sec}$ & $95^{\circ} \mathrm{C}$ \\
Annealing/elongation* & $1 \mathrm{~min}$ & $60^{\circ} \mathrm{C}$ \\
Dissociation stage & $15 \mathrm{sec}$ & $95^{\circ} \mathrm{C}$ \\
& $20 \mathrm{sec}$ & $60^{\circ} \mathrm{C}$ \\
Recording of the & $20 \mathrm{sec}$ & stepwise rising of the \\
dissociation curve & & temperature to $95^{\circ} \mathrm{C}$ \\
$* 40$ cycles &
\end{tabular}

\subsection{Generation of cDNA}

Isolated mRNA from DG75 cells was re-transcribed into cDNA using the first strand cDNA synthesis kit from Fermentas following the manufacturer's instructions.

\subsection{Sequence analysis of DNA}

Cloned DNA fragments were sequenced by seqlab-Microsynth (Göttingen, Germany). Therefore, samples containing $1.2 \mu \mathrm{g}$ DNA and $30 \mathrm{pmol}$ of the appropriate primer in a total volume of $15 \mu \mathrm{Idd}_{2} \mathrm{H}_{2} \mathrm{O}$ were prepared.

\subsubsection{Agarose gel electrophoresis}

For size separation of DNA fragments agarose gel electrophoresis was performed. Agarose concentrations of $1-2 \%$ in TAE buffer were prepared with $0.5 \mu \mathrm{g} / \mathrm{ml}$ final 
ethidium bromide concentration. DNA samples were mixed with $6 \times$ DNA loading buffer, loaded onto the gel and the separation was performed at $220 \mathrm{~mA}$ and $100 \mathrm{~V}$ for $25-45 \mathrm{~min}$ depending on the DNA fragment size. The GeneRuler $1 \mathrm{~kb}$ DNA ladder (Fermentas) was carried along for determination of fragment size and quantity.

TAE buffer:

$40 \mathrm{mM}$ Tris/acetic acid, $\mathrm{pH} 7.8$

$10 \mathrm{mM} \mathrm{NaOAc}$

1 mM EDTA

${ }_{\text {dd }} \mathrm{H}_{2} \mathrm{O}$

$\mathrm{pH} 8$

\subsubsection{Cloning techniques}

\subsubsection{Restriction endonuclease digest}

Cleavage of plasmids, vectors and PCR products were performed with enzymes and buffers from NEB as recommended by the supplier.

For an analytical cleavage, $2 \mu \mathrm{l}$ of DNA was cut with one or two enzymes in the total volume of $15 \mu \mathrm{l}$. Here, $0.3 \mu \mathrm{l}$ of enzyme and $1.5 \mu \mathrm{l}$ of the appropriate $10 \times$ NEB buffer was used and the restriction was performed for at least $1 \mathrm{~h}$ at $37^{\circ} \mathrm{C}$ followed by agarose gel electrophoresis.

For a preparative cleavage, $2 \mu \mathrm{g}$ of insert and vector backbone DNA was cut with $1 \mu \mathrm{l}$ of each enzyme in a total volume of $50 \mu \mathrm{l}$ for at least $3 \mathrm{~h}$ at $37^{\circ} \mathrm{C}$. When only one enzyme was used in the cleavage reaction of the vector backbone, $1 \mu \mathrm{l}$ calf intestine phosphatase was added for the last 30 minutes to dephosphorylate $5^{\prime}$ - and $3^{\prime}$-ends of the linearized vector DNA and thereby prevent religation. After that, DNA purification was performed using agarose gel electrophoresis and gel extraction.

\subsubsection{Purification of DNA}

For DNA purification from agarose gels and PCR reactions the Wizard ${ }^{\circledR}$ SV Gel and PCR Clean-Up System (Promega) according to the manufacturer's instructions was used. Elution of DNA was performed using $30 \mu$ of nuclease free $\mathrm{H}_{2} \mathrm{O}$.

\subsubsection{Ethanol precipitation of DNA}

First, a DNA phenol-chloroform extraction was performed by adding equal volume of phenol-chloroform Isoamyl alcohol (2:3:4) and short centrifugation for separation of the two phases. The DNA containing phase was mixed with $1 / 10$ volume $5 \mathrm{M} \mathrm{NaCl}$ and 2.5 
volumes of $-20{ }^{\circ} \mathrm{C}$ cold $100 \% \mathrm{EtOH}$ with subsequent incubation at $-80^{\circ} \mathrm{C}$ for $20 \mathrm{~min}$. DNA was pelleted at $20000 \times \mathrm{g}, 4^{\circ} \mathrm{C}, 10 \mathrm{~min}$, afterwards dried at air and finally dissolved in PBS.

PBS:

$137 \mathrm{mM} \mathrm{NaCl}$

$2.4 \mathrm{mM} \mathrm{KCl}$

$4.3 \mathrm{mM} \mathrm{Na}_{2} \mathrm{HPO}_{4} \times 12 \mathrm{HPO}_{4}$

$1.4 \mathrm{mM} \mathrm{KH}_{2} \mathrm{PO}_{4}$

$\mathrm{dd}_{2} \mathrm{O}$

$\mathrm{pH} 7.4$

\subsubsection{T/A cloning of PCR fragments}

Amplified and purified PCR fragment, which were generated with the phusion polymerase, were mixed with the Taq polymerase master mix to generate $3^{\prime}-\mathrm{A}$ overhangs. Like this, the amplified PCR fragment could be ligated into a linearized pCR2.1 vector according to the manufacturer's instructions.

\subsubsection{Ligation of DNA fragments}

Cleaved, purified inserts and vector backbones with compatible sticky ends were used in a T4 Ligase (New England Biolabs ${ }^{\circledR}$ ) mediated ligation reaction. Therefore, a ratio of insert to vector of 3:1 was used together with $0.5 \mu \mathrm{l}$ T4 ligase and $1 \mu \mathrm{l}$ ligase buffer in a total volume of $10 \mu \mathrm{l}$. Additionally, a control reaction lacking the insert DNA was set up to determine the re-ligation events of the vector backbone. The reactions for short insert fragments were performed for at least $3 \mathrm{~h}$ at room temperature or overnight at $16{ }^{\circ} \mathrm{C}$ for longer fragments. The ligation reaction was subsequently used for the transformation of competent E.coli and plated on the corresponding selective LB-plates.

\subsubsection{Preparation of chemically competent E.coli}

For expression of plasmids, the different E.coli strains were made chemically competent and were kindly provided by Ines Heine.

\subsubsection{Transformation of chemically competent E.coli}

Chemically competent E.coli $(50 \mu \mathrm{l})$ were thawed on ice and the whole ligation reaction or for a retransformation $0.5 \mu \mathrm{l}$ of the DNA were added to the bacteria and mixed by flicking 
of the tube. Incubation on ice for 20 min was followed by a heat shock at $42{ }^{\circ} \mathrm{C}$ for $50 \mathrm{sec}$ and subsequent incubation on ice for 2 min. Afterwards, $150 \mu \mathrm{l}$ LB medium without antibiotic was added and the cells were grown at $37^{\circ} \mathrm{C}$ for 30 min before the whole transformation mix was spread out onto LB-plates containing the appropriate antibiotics. The E.coli were incubated at $37^{\circ} \mathrm{C}$ overnight.

In the case of E.coli transformation with TALEN plasmids or pCR2.1 plasmids, blue-white screening was performed. To this end, $50 \mu \mathrm{l}$ of Isopropyl- $\beta$-D-thiogalactopyranoside (IPTG) $(0.1 \mathrm{M})$ and $50 \mu \mathrm{l}$ of 5-bromo-4-chloro-3-indolylbeta-D-galacto-pyranoside (X-Gal) (50 $\mathrm{mg} / \mathrm{ml}$ in DMF) had been distributed on the LB-plate before the transformation mix was added.

\subsubsection{Biochemistry}

\subsubsection{Preparation of cleared cellular lysates}

To analyze protein expression levels of DT40 and human cells, cleared cellular lysates $(C C L)$ were prepared and either used for analyses with immunoblotting or further used in affinity purification (AP) experiments. For the preparation of CCL, $1 \times 10^{6}-5 \times 10^{7}$ resting or BCR-stimulated cells were pelleted and the supernatant was discarded. $1 \times 10^{6}$ cells were resuspended in $20 \mu \mathrm{l}$ lysis buffer. Lysis was performed on ice for $30 \mathrm{~min}$ with subsequent centrifugation at maximum speed for $10 \mathrm{~min}$ at $4{ }^{\circ} \mathrm{C}$. CCL were transferred to another microcentrifuge tube, mixed with $1 / 4$ 4x Laemmli buffer, heated for 5 min at $95{ }^{\circ} \mathrm{C}$ and stored at $-20^{\circ} \mathrm{C}$.

Lysis buffer:

$50 \mathrm{mM}$ Tris/HCl, $\mathrm{pH} 8$

$150 \mathrm{mM} \mathrm{NaCl}$

$5 \mathrm{mM} \mathrm{NaF}$

$1 \mathrm{mM} \mathrm{Na}_{3} \mathrm{VO}_{4}$

protease inhibitor cocktail

$0.5 \%$ NP-40

Laemmli buffer: $\quad 250 \mathrm{mM}$ Tris/HCl, $\mathrm{pH} 6.8$

200 mM DTT

$40 \%$ glycerol

$8 \%$ SDS

$0.05 \%$ bromophenol blue 


\subsubsection{Stimulation of $B$ cells}

Chicken and human B cells were collected, washed with PBS (300 x g, $\left.4^{\circ} \mathrm{C}, 4 \mathrm{~min}\right)$ and resuspended in RPMI without FCS (w/o). The cells were starved at $37^{\circ} \mathrm{C}$ for at least 30 min and stimulated via the BCR:

DT40: $\quad 2 \mu \mathrm{g} / \mathrm{ml}$ anti-chicken $\operatorname{lgM}(\mathrm{M} 4)$

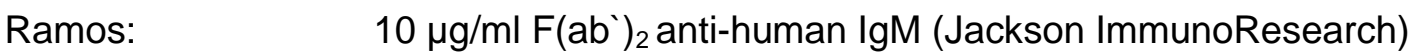

DG75: $\quad 1-10 \mu \mathrm{g} / \mathrm{ml} \mathrm{F}\left(\mathrm{ab}^{\prime}\right)_{2}$ anti-human IgM (SouthernBiotech)

Y2a expressing cells: $10 \mu \mathrm{g} / \mathrm{ml} \mathrm{F}\left(\mathrm{ab}^{\prime}\right)_{2}$ anti-mouse IgG (Jackson ImmunoResearch)

For BCR independent stimulation of $B$ cells the cells were treated with pervanadate $(1: 100)$ for $5 \mathrm{~min}$.

Stimulations were performed at $37^{\circ} \mathrm{C}$ for desired time points and stopped by immediate centrifugation of the samples with $950 \times \mathrm{g}$, at $4^{\circ} \mathrm{C}$, for $2 \mathrm{~min}$.

Pervanadate: $\quad 100 \mu$ of $50 \mathrm{mM} \mathrm{Na}_{3} \mathrm{VO}_{4}$

$30 \mu \mathrm{l}$ of $30 \% \mathrm{H}_{2} \mathrm{O}_{2}$

$520 \mu \mathrm{l} \mathrm{H}_{2} \mathrm{O}$

\subsubsection{Expression and purification of recombinant GST-fusion proteins}

Plasmids coding for GST-fusion proteins were transformed into the E.coli strain BL21 and plated on LB-agar plates with ampicillin for overnight incubation at $37^{\circ} \mathrm{C} .4 \mathrm{ml} \mathrm{LB}$ medium were inoculated with one BL21 colony, incubated overnight shaking at $37^{\circ} \mathrm{C}$. The following day $100 \mathrm{ml}$ YT-medium supplemented with ampicillin were inoculated with $2 \mathrm{ml}$ of the overnight culture. The GST-fusion protein expression was induced with $100 \mu \mathrm{M}$ IPTG for $4 \mathrm{~h}$ shaking at $37^{\circ} \mathrm{C}$ when the $\mathrm{OD}_{600}$ of 0.6 was reached. In the end of the expression, the bacteria were pelleted in $50 \mathrm{ml}$ tubes by centrifugation at $3000 \times \mathrm{g}, 4^{\circ} \mathrm{C}$ for $20 \mathrm{~min}$. Harvested bacteria of $50 \mathrm{ml}$ culture were resuspended in $2 \mathrm{ml}$ PBS and lysed by sonification on ice ( $2 \times 15 \mathrm{sec}, 5$ cycles, $50 \%)$. Full lysis was achieved by addition of TritonX-100 in a final concentration of $0.5 \%$ with subsequent incubation on ice for $30 \mathrm{~min}$. The lysate was spun down at $5000 \times \mathrm{g}$ at $4{ }^{\circ} \mathrm{C}$ for $20 \mathrm{~min}$. Consequently, the supernatant was transferred to a $2 \mathrm{ml}$ microcentrifuge tube and $100 \mu \mathrm{l}$ equilibrated glutathione sepharose was added and incubated for $2 \mathrm{~h}$ at $4{ }^{\circ} \mathrm{C}$ on a rotating wheel. The glutathione sepharose was washed twice with PBS ( $\left.250 \times \mathrm{g}, 3 \mathrm{~min}, 4^{\circ} \mathrm{C}\right)$ and in the end used for affinity purification experiments. 


\subsubsection{Affinity purification}

During this project, I performed different types of affinity purifications (APs) with either directly coupled beads or with precipitating antibodies, followed by immobilization with beads, to purify proteins.

For affinity purifications with GST-fusion proteins, $10 \mu \mathrm{g}$ of the GST-fusion proteins immobilized on glutathione sepharose were added to the CCL of $3-5 \times 10^{7}$ cells and incubated on a rotating wheel at $4{ }^{\circ} \mathrm{C}$ for at least $2 \mathrm{~h}$. In the case of protein purification with ubiquitin coupled to agarose, $15 \mu \mathrm{l}$ of the equilibrated suspension were added to the $\mathrm{CCL}$ of $5 \times 10^{7}$ cells and incubated on a rotating wheel at $4{ }^{\circ} \mathrm{C}$ for $4 \mathrm{~h}$.

For the purification of the BCR, chicken DT40 cells were stimulated with $2 \mu \mathrm{g} / \mathrm{ml} \mathrm{M} 4$ for different time points at $37^{\circ} \mathrm{C}$, kept on ice for the unstimulated sample or treated with pervanadate. Samples were washed once with PBS to remove unbound stimulating antibody and lysed with cell lysis buffer. Cleared cellular lysates were transferred to a new microcentrifuge tube and goat anti-mouse IgM was added for one hour with subsequent incubation on a rotating wheel. For an additional $45 \mathrm{~min}$ incubation period, $20 \mu \mathrm{l}$ equilibrated protein $A / G$ beads were used for immobilization. After three times washing of the beads with $600 \mu \mathrm{l}$ cell lysis buffer ( $250 \mathrm{xg}, 4^{\circ} \mathrm{C}, 3 \mathrm{~min}$ ), $50 \mu \mathrm{l} 4 \mathrm{x}$ laemmli buffer was added and the samples were incubated at $95^{\circ} \mathrm{C}$ for $5 \mathrm{~min}$.

In the case of $y 2 a-B C R$ purifications from Ramos and DG75 cells, the stimulation was performed with $10 \mu \mathrm{g} / \mathrm{ml}$ anti-mouse IgG-BIOTIN for different time points at $37{ }^{\circ} \mathrm{C}$ or on ice for the unstimulated sample. After washing once with PBS and lysis with cell lysis buffer, $15 \mu \mathrm{l}$ equilibrated streptavidin sepharose were used for immobilization. Samples were incubated for $1 \mathrm{~h}$ and washed three times with $600 \mu$ cell lysis buffer. $50 \mu \mathrm{l} 4 \mathrm{x}$ laemmli buffer were added to the beads and heated up for 5 min at $95^{\circ} \mathrm{C}$.

\subsubsection{SDS-polyacrylamide gel electrophoresis}

To separate proteins by size, discontinuous gel electrophoresis was performed like described by Laemmli (Laemmli, 1970). The usage of the Laemmli sample buffer caused the proteins to be denaturated. For the separation by size a $5 \%$ stacking gel and a $10 \%$ separating gel were applied. Electrophoresis was performed with a current of $15 \mathrm{~mA}$ for the stacking and $25 \mathrm{~mA}$ for the separating gel. As a molecular weight standard the prestained protein marker of NEB was used. The SDS-PAGE was then either stained with coomassie blue or used for immunoblotting to visualize the proteins. 
Stacking gel:

$125 \mathrm{mM}$ Tris/HCl, $\mathrm{pH} 6.8$

$4.8 \%$ acrylamide

$0.1 \%$ TEMED

$0.1 \%$ APS

Separating gel:

$375 \mathrm{mM}$ Tris/ $\mathrm{HCl}, \mathrm{pH} 8.8$

$10 \%$ acrylamide

$0.1 \%$ TEMED

$0.1 \%$ APS

SDS running buffer:

$25 \mathrm{mM}$ Tris

$192 \mathrm{mM}$ glycine

$0.1 \%(w / v)$ SDS

Coomassie staining solution: $2.5 \mathrm{~g} / \mathrm{L}$ Coomassie Brilliant Blue R250

$45 \% \mathrm{MeOH}$

$10 \%$ acetic acid

\subsubsection{Western blotting}

The proteins that were separated on the SDS-gel were transferred onto a nitrocellulose membrane to make them visible with immunostaining (Towbin et al., 1979). A semi-dry system was used where two slices whatman paper, a nitrocellulose membrane and a SDS-gel were soaked in blotting buffer. They were layered on the anode of the blotting chamber in a stack of whatman paper, nitrocellulose membrane, SDS-PAGE and again whatman paper. Potential air bubbles were removed by rolling with a pipette and the transfer was performed with $16 \mathrm{~V}$ for $70 \mathrm{~min}$. The membrane was then incubated with blocking solution for at least $1 \mathrm{~h}$ shaking at room temperature. Proteins were made visible with applying the primary antibody solution overnight at $4{ }^{\circ} \mathrm{C}$ followed by the corresponding HRPO-coupled secondary antibodies in a 1:10000 dilution in TBS-T for $1 \mathrm{~h}$ at RT. For visualizing of the proteins immunostaining with $4 \mathrm{ml} \mathrm{ECL}$ solution was used and the signal was detected with the Chemilux Imaging system by Intas.

Blotting buffer: $\quad 39 \mathrm{mM}$ glycine

$48 \mathrm{mM}$ Tris/Hcl

$0.0375 \%(w / v)$ SDS

$20 \% \mathrm{MeOH}$ 
TBS-T:

$25 \mathrm{mM}$ Tris/HCl, $\mathrm{pH} 8.0$

$125 \mathrm{mM} \mathrm{NaCl}$

$0.1 \%$ Tween20

in $\mathrm{dd}_{2} \mathrm{O}$

adjust to $\mathrm{pH} 7.4$

Blocking solution:

$5 \%(w / v)$ BSA in TBS-T

ECL solution:

$4 \mathrm{ml}$ solution $\mathrm{A}: 250 \mathrm{mg} / \mathrm{L}$ luminol in $0.1 \mathrm{M}$ Tris $/ \mathrm{HCl}, \mathrm{pH} 8.6$

$400 \mu \mathrm{l}$ solution B: $55 \mathrm{mg}$ para-Coumaric acid in $50 \mathrm{ml}$ DMSO

$1.2 \mu \mathrm{l}$ of $30 \% \mathrm{H}_{2} \mathrm{O}_{2}$

\subsubsection{Cell biology}

\subsubsection{Cell culture medium}

For the cultivation of the human B cell lines Ramos and DG75, RPMI 1640 (+GlutaMax) purchased from Biochrome was used, which was supplemented with $10 \%$ fetal calf serum (FCS) and $1 \%$ Penicillin/Streptomycin (R10). In the case of cultivation of the chicken DT40 B cell line, R10 with additional $1 \%$ chicken serum (CS) was used. For the adherent cell line PlatE, DMEM from Biochrome was used, which was also supplemented with $10 \%$ FCS and $1 \%$ Penicillin/Streptomycin. To inactivate complement factors, FCS and CS were heated to $56^{\circ} \mathrm{C}$ for $30 \mathrm{~min}$.

\subsubsection{Eukaryotic cell lines}

DG75 (DSMZ-No: ACC 83)

This human B cell model cell line is derived from a pleural diffusion of a 10-year-old boy with a burkitt lymphoma (refractory, terminal) in 1975. It is a male, diploid B cell line with the translocation: $t(8 ; 14)(q 24 ; q 32)$ (Ben-Bassat et al., 1977).

Table 2.13: DG75 knock out sub-lines used in this project.

\begin{tabular}{l|l} 
DG75 & REFERENCE \\
\hline CIN85 & This work \\
SLP65 & (Hitzing, 2015) \\
CIN85/SLP65 & This work
\end{tabular}

\section{Ramos (DSMZ-No: ACC 603)}

The Ramos B cell line is established from the ascetic fluid of a 3-year-old boy with burkitt lymphoma in 1972 (Klein et al., 1975). 


\section{DT40 (ATCC ${ }^{\circledR}$ Number: CRL-2111 ${ }^{\mathrm{TM}}$ )}

The chicken DT40 B cell line is an avian leucosis virus-induced bursal lymphoma cell line (Baba and Humphries, 1984). Since this cell line shows an increased ratio of targeted to random DNA integration, it constitutes a good model system for homologous recombination (Winding and Berchtold, 2001).

Table 2.14: DT40 knock out sub-lines used in this project.

\begin{tabular}{l|l} 
DT40 & REFERENCE \\
\hline$S L P 65^{--}$ & (Ishiai et al., 1999) \\
$L Y N^{-}$ & (Takata et al., 1994) \\
$C D 2 A P^{--}$ & (Bremes, 2012)
\end{tabular}

\section{Platinum-E}

The Platinum-E (PlatE) cell line is HEK 293T-derived and used for retroviral packaging (Morita et al., 2000). It expresses gag, pol and env proteins and can be transiently transfected with a plasmid coding for the glycoprotein of the vesicular stromatitis virus (VSV-G) to allow efficient infection of human and chicken B cell lines.

\subsubsection{Cell culture}

The non-adherent cell lines DG75 and Ramos were grown in culture dishes using R10 and they were incubated in the HeraCell 150 incubator with $5 \% \mathrm{CO}_{2}$ at $37^{\circ} \mathrm{C}$. The cells were split 1:10 every second day. The non-adherent cell line DT40 was cultured under the same conditions with the exceptions that R10 was supplemented with $1 \%$ CS and that the cells were split 1:10 every day.

The adherent cell line PlatE was grown in D10 and passed 1:10 every second day. Therefore, the medium was removed, the cells were washed with PBS and $0.05 \%$ Trypsin/EDTA was added to detach the cells.

\subsubsection{Freezing and thawing of eukaryotic cells}

Approximately $1 \times 10^{7}$ cells were harvested ( $300 \times \mathrm{g}, \mathrm{RT}, 4 \mathrm{~min}$ ), resuspended in $1 \mathrm{ml}$ freezing medium, transferred into cryo-tubes and immediately placed on ice. For long-term storage, the cells were stored at $-150^{\circ} \mathrm{C}$.

The cells were put on $37^{\circ} \mathrm{C}$ for rapid thawing and taken up in $10 \mathrm{ml}$ culture medium. Following centrifugation at $300 \times \mathrm{g}$, RT for 4 min the cells were resuspended in $10 \mathrm{ml}$ fresh culture medium and transferred into a culture dish for cultivation. 
Freezing medium: $\quad 90 \%$ FCS (v/v)

$10 \%$ dimethyl sulfoxide (DMSO) (v/v)

\subsubsection{CHX and MG132 treatment}

For analysis of protein turnover rates, cells were treated with $25 \mu \mathrm{g} / \mathrm{ml}$ cycloheximide $(\mathrm{CHX})$ and $20 \mu \mathrm{M}$ MG132 for the indicated time points. As both reagents were dissolved in DMSO, this solvent served as negative control in the experiments.

$1 \times 10^{6}$ cells per sample were cultured in 12 -well plates at $37^{\circ} \mathrm{C}$ for at least $20 \mathrm{~min}$ in prewarmed R10 before the treatment was initiated. The corresponding amounts of reagents were added into the culture and distributed via smoothly shaking. Hereafter, the cells were incubated at $37{ }^{\circ} \mathrm{C}$ starting with the longest time point and ending with the shortest. To stop the incubation period, the cells were transferred into tubes and were placed on ice. After determination of cell numbers of the different samples, cleared cellular lysates were prepared and analyzed via immunoblotting.

\subsubsection{Transfection methods}

\subsubsection{Electroporation}

For electroporation, pABES plasmids were used. cDNA was linearized with Pvul, which cleaves in the ampicillin resistance cassette, in a total volume of $400 \mu \mathrm{l}$ at $37^{\circ} \mathrm{C}$ for at least $3 \mathrm{~h}$. For transfection of DT40 $25 \mu \mathrm{g}$ and for human cells $50 \mu \mathrm{g}$ linearized cDNA was used. Total digest was confirmed on an agarose gel. DNA phenol-chloroform extraction was performed with the linearized cDNA. $2 \times 10^{7}$ cells were resuspended in $700 \mu \mathrm{PBS}$ containing the cDNA and transferred into an electroporation cuvette. After incubation on ice for 10 min, chicken DT40 cells were electroporated with $270 \mathrm{~V}, 960 \mu \mathrm{FD}$ whereas the settings for human cells were $250 \mathrm{~V}$ and $960 \mu \mathrm{FD}$. After subsequent incubation on ice for $10 \mathrm{~min}$, the cells were plated in $10 \mathrm{ml}$ warm culture medium and incubated at $37^{\circ} \mathrm{C}$ for 24 $\mathrm{h}$ to recover. This was followed by centrifugation ( $300 \mathrm{xg}, \mathrm{RT}, 4 \mathrm{~min})$ and resuspension of the transfected cells in $40 \mathrm{ml}$ fresh culture medium supplemented with the appropriate antibiotic for selection. The cells were sub-cloned on two 96-well plates until colonies were forming and the clones could be expanded and analyzed for expression of the desired construct via immunoblotting. 


\subsubsection{Retroviral transfection}

PlatE cells, 50-70\% confluent and seeded in $6 \mathrm{~cm}$ dishes, were used for viral transduction of chicken DT40 and human B cells. Therefore, $1.8 \mu \mathrm{g}$ for DT40 and $4 \mu \mathrm{g}$ retroviral expression vector for human cells were incubated with $7.5 \mu \mathrm{l}$ Trans-IT, $0.7 \mu \mathrm{g}$ pHCMVVSV-G and $180 \mu \mathrm{l} \mathrm{RPMI} \mathrm{(w/o)} \mathrm{for} 30 \mathrm{~min}$ at RT. D10 was removed from the PlatE cells and $2.5 \mathrm{ml}$ fresh R10 was added. After the incubation period, the lipofection mix was added dropwise onto the PLatEs and following $24 \mathrm{~h}$ of incubation at $37{ }^{\circ} \mathrm{C}$ and $5 \% \mathrm{CO}_{2}, 2$ $\mathrm{ml}$ of fresh R10 were added for another $24 \mathrm{~h}$ incubation. In this time, the PlatEs produced retroviruses and secreted them into the supernatant. This supernatant was collected and eventually present PlatE cells were pelleted at $300 \times \mathrm{g}, \mathrm{RT}$, for $4 \mathrm{~min}$. $1 \times 10^{6}$ DT40 or $2 \times 10^{6}$ human cells were resupended in $2 \mathrm{ml}$ of the respective culture medium and $4 \mathrm{ml}$ of the cleared virus containing supernatant were added for infection. Infection efficiency was increased by using a final concentration of $3 \mu \mathrm{g} / \mathrm{ml}$ polybrene dissolved in PBS, which was sterile filtered ( $0.2 \mu \mathrm{m}$ pore size). After $24 \mathrm{~h}$, polybrene was removed from the cells by centrifugation at $300 \times \mathrm{g}, \mathrm{RT}$ and $4 \mathrm{~min}$ with subsequent uptake in fresh culture medium. Selection with the appropriate antibiotics was started another $24 \mathrm{~h}$ later.

DT40: $\quad$ puromycin $1 \mu \mathrm{g} / \mathrm{ml}$ for $72 \mathrm{~h}$

blasticidin $30 \mu \mathrm{g} / \mathrm{ml}$ for $72 \mathrm{~h}$

bleocin $70 \mu \mathrm{g} / \mathrm{ml}$ for $48 \mathrm{~h}$

Human cells: puromycin $2 \mu \mathrm{g} / \mathrm{ml}$ for $72 \mathrm{~h}$

blasticidin $20 \mu \mathrm{g} / \mathrm{ml}$ for $72 \mathrm{~h}$

bleocin $50 \mu \mathrm{g} / \mathrm{ml}$ for $48 \mathrm{~h}$

\subsubsection{Transfection via nucleofection}

DG75 cells were transfected with the Human B cell Nucleofector ${ }^{\circledR}$ Kit (Lonza). $2 \times 10^{6}$ DG75 cells were collected and centrifuged at $90 \times \mathrm{g}, \mathrm{RT}$, for $10 \mathrm{~min}$. The supernatant was removed completely and the cells were resuspended in the transfection mix. This mixture contained $82 \mu$ l Human B Cell Nucleofector ${ }^{\circledR}$ Solution, $18 \mu$ l Supplement 1 and $2 \mu \mathrm{g}$ of each plasmid. The cells were immediately transferred to the included nucleofection cuvettes and nucleofection was performed with program T-015 using the Amaxa Nucleofectorll ${ }^{\mathrm{TM}}$ device. Afterwards, the cells were cultured in a 6-well plate with $2 \mathrm{ml}$ prewarmed R10. 


\subsubsection{Flow cytometry}

\subsubsection{Analysis of fluorophore expression}

To analyze expression of ectopically expressed fluorophore-tagged proteins, $0.5 \times 10^{6}$ cells were harvested (300 x g, RT, 4 min), resuspended in $300 \mu \mathrm{lPBS}$ and examined with the flow cytometer.

\subsubsection{Analysis of BCR surface expression}

To test for surface expression of either endogenous IgM or transduced lgG, $0.5 \times 10^{6}$ cells were washed with cold PBS ( $300 \times \mathrm{g}, 4^{\circ} \mathrm{C}, 4 \mathrm{~min}$ ) and stained with $0.5 \mu \mathrm{l}$ anti-human lgMAPC $(0.1 \mathrm{mg} / \mathrm{ml})$ or anti-mouse $\operatorname{lgG}_{2 a}-\mathrm{CY} 5(1 \mathrm{mg} / \mathrm{ml})$ in $100 \mu \mathrm{l}$ PBS on ice and in the dark for $15 \mathrm{~min}$. Unbound antibodies were washed away with cold PBS ( $300 \times \mathrm{g}, 4^{\circ} \mathrm{C}, 4 \mathrm{~min}$ ) and the cells were resuspended in $300 \mu$ cold PBS for subsequent flow cytometry analysis.

\subsubsection{BCR internalization assay}

To analyze residual amounts of surface $\operatorname{lgM}$ or $\lg G$ after BCR stimulation, $1 \times 10^{6}$ cells were used per sample. They were washed once with cold PBS (300 x g, $\left.4{ }^{\circ} \mathrm{C}, 4 \mathrm{~min}\right)$, resuspended in $100 \mu \mathrm{l}$ PBS and $1 \mu \mathrm{l}$ biotinylated anti-human IgM $(0.5 \mathrm{mg} / \mathrm{ml})$ or antimouse $\lg G(0.5 \mathrm{mg} / \mathrm{ml})$ was added for a $15 \mathrm{~min}$ incubation on ice. Unbound antibody was washed away with cold PBS $\left(300 \times \mathrm{g}, 4^{\circ} \mathrm{C}, 4 \mathrm{~min}\right)$ and the cells were taken up in KrebsRinger solution. For stimulation/internalization, the cells were incubated at $37^{\circ} \mathrm{C}$ for the different time points or left on ice for the unstimulated sample. The stimulation process was stopped by adding $2 \mathrm{ml}$ of cold PBS and placing the samples on ice immediately. After washing the cells $\left(300 \times \mathrm{g}, 4^{\circ} \mathrm{C}, 4 \mathrm{~min}\right)$, they were resuspended in $100 \mu \mathrm{l}$ PBS and stained with $0.5 \mu \mathrm{l}$ APC-conjugated streptavidin $(0.2 \mathrm{mg} / \mathrm{ml})$ on ice and in the dark, that bind to the remaining biotinylated surface BCRs. Unbound streptavidin was washed away with cold PBS ( $300 \times \mathrm{g}, 4^{\circ} \mathrm{C}, 4 \mathrm{~min}$ ) and the cells were resuspended in $300 \mu \mathrm{l}$ PBS for subsequent analysis by flow cytometry.

Krebs-Ringer solution : $\quad 10$ mM HEPES, $\mathrm{pH} 7.0$

$140 \mathrm{mM} \mathrm{NaCl}$

$4 \mathrm{mM} \mathrm{KCl}$

$1 \mathrm{mM} \mathrm{MgCl} 2$

$10 \mathrm{mM}$ glucose

$1 \mathrm{mM} \mathrm{CaCl}_{2}$ 


\subsubsection{Cell sorting}

Cell sorting was performed in cooperation with the cell sorting facility of the university medical center Göttingen, AG Wulf. Cells that were sorted for my2a surface expression were stained with an anti-mouse $\operatorname{lgG}_{2 a}-\mathrm{CY} 5(1 \mathrm{mg} / \mathrm{ml}$ ) (see chapter 2.2.6.2). Whereas cells that were sorted for citrine expression could be used directly. In both cases, approximately $2 \times 10^{6}$ cells were washed once with PBS $\left(300 \times \mathrm{g}, 4^{\circ} \mathrm{C}, 4 \mathrm{~min}\right)$, taken up in $500 \mu \mathrm{l}$ PBS and stored on ice until sorting with a BD Aria. The living and positive sorted cells were taken up in R10 and washed once with R10 before they were cultured.

\subsubsection{5 $\mathrm{Ca}^{2+}$ mobilization assay}

To analyze $\mathrm{Ca}^{2+}$ mobilization in $\mathrm{B}$ cells by flow cytometry, the $\mathrm{Ca}^{2+}$ sensitive dye Indo-1AM, a polycyclic chelator, was used. It changes fluorescence emission from $475 \mathrm{~nm}$ in the $\mathrm{Ca}^{2+}$ unbound state to $400 \mathrm{~nm}$ in the $\mathrm{Ca}^{2+}$ bound state. This change in fluorescence emission was monitored with the cytometer LSRII. Therefore, $2 \times 10^{6}$ cells were taken up in $600 \mu \mathrm{l} 10$ and stained by addition of $0.7 \mu \mathrm{l}$ Indo-1-AM and $2.1 \mu \mathrm{l}$ pluronic F-127. For the staining process, the cells were transferred to light protected tubes and first incubated at $30{ }^{\circ} \mathrm{C}$ for $20 \mathrm{~min}$ with subsequent incubation at $37^{\circ} \mathrm{C}$ for $10 \mathrm{~min}$, both shaking. After washing twice with Krebs-Ringer solution (300 $\mathrm{g}$ g, RT, $4 \mathrm{~min}$ ), the cells were taken up in $600 \mu \mathrm{Krebs}-$ Ringer and incubated at $30{ }^{\circ} \mathrm{C}$ for $40 \mathrm{~min}$. Duplicates were measured in 300 $\mu \mathrm{l}$ Krebs-Ringer each. $20 \mathrm{sec}$ after the start of recording, the stimulating antibodies were added and the fluorescence emission was measured for 4-5 min. $\mathrm{Ca}^{2+}$ mobilization profiles were analyzed with the Flowjo 7.6.4 software.

Indo-1-AM stock: $\quad 1 \mathrm{mM}$ in dried $\mathrm{DMSO}$, stored at $-20^{\circ} \mathrm{C}$

Pluronic F-127 stock: $\quad 5 \%(w / v)$

\subsubsection{Confocal laser scanning microscopy}

Confocal laser scanning microscopy was used to visualize citrine-, GFP- or RFP-tagged proteins in the living resting or BCR-stimulated cells. To this end, $2 \times 10^{6}$ cells were harvested and washed twice with Krebs-Ringer. The cells were resuspended in $800 \mu \mathrm{l}$ Krebs-Ringer and duplicates were measured in $400 \mu \mathrm{l}$ each in a 4-well imaging slide. After approximately $20 \mathrm{~min}$ in which the cells settle down, they were analyzed at the Leica TCS SP2 confocal laser scanning microscope in the department of the medical microbiology and infection immunology in the working group of Prof. Dr. Carsten Lüder. The cells were 
stimulated with different amounts of anti-chicken $\lg \mathrm{M}$ as well as anti-human IgM or antimouse $\left.\lg G \mathrm{~F}(\mathrm{ab})_{2}\right)_{2}$ fragments and scans were taken after varying time points.

\subsubsection{Gene targeting and interference}

\subsubsection{RNA interference with microRNA-30 adapted shRNA-mir retroviral vectors}

Gene of interest expression was suppressed by shRNA mediated RNA interference. To do this, the microRNA-30 adapted shRNA-mir system from Open Biosystems (Expression Arrest microRNA-adapted Retroviral Vector) was used. Therefore, the target sequence in the gene of interest was chosen in a way that it contains the sense targeting sequence, a miR-30 loop structure and the antisense targeting sequence, flanked $5^{\prime}$ and $3^{\prime}$ by a mirR30 context. This oligonucleotide was amplified in a PCR reaction to add restriction sites and then cloned into the LMP pMSCV GFP backbone for retroviral transduction. As a control, an unspecific sh mock sequence was used. The shRNA was introduced into the cells by retroviral transduction.

\subsubsection{Transcription Activator-like Effector Nucleases - TALEN}

For generation of a stable CIN85-deficient human B cell sub-line, the transcription activator like effector nucleases (TALEN) method was applied. TALEN allows the generation of knock out cell lines due to transient expression of constructs coding for proteins that provide a DNA binding segment and an endonuclease, which introduces double-strand breaks. The TALEN constructs were generated with the plasmid kit that was a gift from Daniel Voytas and Adam Bogdanove (Addgene kit \# 1000000024). The cloning of TALEN constructs was done according to the manufacturer's protocol and the modifications established by Christoffer Hitzing (Hitzing, 2015). Exon 4 of the human CIN85 gene locus (ENSG00000147010) was chosen for targeting with the TALEN constructs as it is present in all CIN85 isoforms and the TALEN targeter software was used to design the TALEN constructs. The TALEN constructs were chosen to contain 1518 DNA binding modules with a 17 bp spacer region between the two binding sites. For convenient activity screening, the TALEN cleavage site was chosen to overlap with an endonuclease binding/restriction site.

The left construct for targeting CIN85 exon 4 binds to the sequence:

5' TACCTGCCCCAGAATG 3'

The right construct for targeting CIN85 exon 4 binds to the sequence:

5' AAAGTTGGCGACATCA 3' 
For transient expression of the TALEN proteins in the human B cell line DG75, the pmax vector with either an IRES-EGFP or an IRES-tagRFP site was used. The construct were introduced into the cells via nucleofection. 36-48 $\mathrm{h}$ after nucleofection, the cells were sorted for EGFP/tagRFP expression in the cell sorting facility of the university medical center. After recovery of the double-positive cells, $1 \times 10^{6}$ cells were used in a TALEN activity test. Therefore, genomic DNA was isolated, the region of the TALEN targeted exon 4 was amplified by PCR, purified and cut with the endonuclease BpuEl for activity testing. When the TALEN constructs were active, the restriction of exon 4 region was decreased and the cells were sub-cloned onto 96-well plates for analysis of protein expression, after reaching sufficient cell numbers. 


\section{RESULTS}

\subsection{Identification of two CIN85-deficient patients}

Recently two male patients with decreased amounts of either total serum antibodies or particular $\operatorname{lgM}$ and $\operatorname{lgG}_{4}$ antibodies were identified by $\mathrm{Dr}$. Moneef Shoukier from the Institute of Human Genetics in Göttingen. The immunological phenotype of these patients was analyzed in cooperation with Dr. Bärbel Keller from the working group of Prof. Dr. Klaus Warnatz from the Center for Chronic Immunodeficiency in Freiburg. The patients are siblings and have a $247.5 \mathrm{kbp}$ deletion in the SH3KBP1 gene, located on chromosome Xp 22.12, which is coding for CIN85. Furthermore, it is important to note that neighboring genes are unaffected. To test whether this deletion results in the loss of CIN85 protein, I prepared cleared cellular lysates of fibroblasts that were kindly provided by the Institute of Human Genetics. I compared cleared cellular lysates of one patient, his mother, a female and male healthy control, respectively. The lysates were separated by SDS-PAGE and the immunoblot was stained with anti-CIN85, anti-CD2AP and anti-Actin as loading control (figure 3.1). The fibroblasts of the patient lacked CIN85 expression whereas the fibroblasts of the hemizygous mother expressed CIN85 to a similar extent as the female control. For both, the patient and the mother, the CIN85 homolog CD2AP was not affected in its expression magnitude. Both brothers came into the clinics because of recurrent infections, which led to the death of the older brother. Analyses of the younger brother's B cells revealed reduced $\mathrm{Ca}^{2+}$ mobilization and NF-KB activation upon BCR stimulation. In contrast, the patients $T$ cells reacted comparable to the cells from the healthy controls (unpublished data). These results highlight the importance of CIN85 in BCR-induced signaling and the need to further elucidate its functional mechanisms.

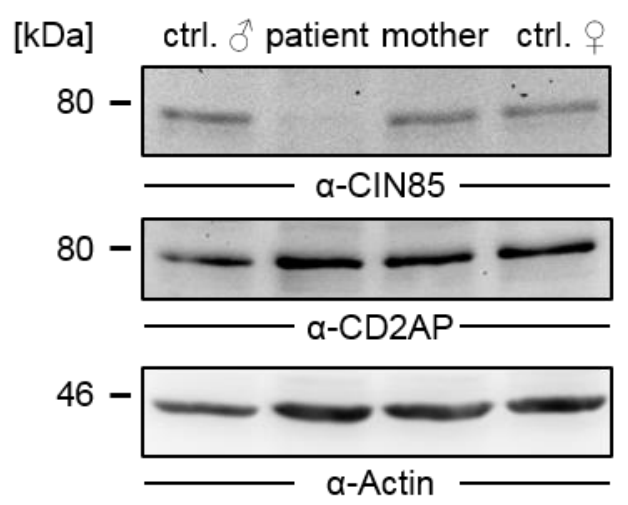

Figure 3.1: Identification of a CIN85-deficient patient. Cleared cellular lysates of fibroblasts from the CIN85-deficient patient, his mother and a female $(+\phi)$ and male $(\widehat{\jmath})$ healthy control (ctrl.) were subjected to SDS-PAGE and the immunoblot was stained with anti-CIN85, anti-CD2AP and anti-Actin antibodies. The respective molecular weight of marker proteins (in $\mathrm{kDa}$ ) is indicated on the left. 


\subsection{Human CIN85 regulates proximal BCR signaling}

3.2.1 Transcription Activator-like Effector Nucleases- (TALEN-) mediated CIN85 inactivation in the human B cell line DG75

In a CIN85-deficient patient the adaptor protein CIN85 was shown to be important for human $B$ cells. Since this deficiency led to abolished serum $\lg M$ and $\lg _{4}$ levels in the patient, we wanted to analyze CIN85's specific functions in detail. Therefore, usage of a cell line is beneficial because it allows genetical and biochemical experiments that are hard to perform in primary cells. Former work of our group intended to generate a cell line deficient for CIN85. However, a putative CIN85-- DT40 cell sub-line generated by homologous recombination (Konstantin Neumann) still showed residual expression of CIN85 isoforms. In a second approach, Vanessa Bremes established a protocol to use mircroRNA-30 adapted shRNA interference against chicken CIN85 (Bremes, 2012). Nevertheless, also with this method some CIN85 expression remained in the DT40 B cells.

To overcome this unwanted residual CIN85 expression, I used the Transcription Activatorlike Effector Nucleases (TALEN) technology (Cermak et al., 2011; Sanjana et al., 2012) to generate a CIN85-deficient human DG75 B cell sub-line, which allows better comparison with the patients' B cells than the evolutionary more distant DT40 chicken B cells. TALEN gene targeting was established by Christoffer Hitzing in our lab (Hitzing, 2015). As the human burkitt lymphoma cell line DG75 originates from a boy and the CIN85 gene is located on the X-chromosome, only one allele has to be targeted for efficient gene inactivation.

For the design of the TALEN constructs, exon 4 of the human CIN85 gene (Chromosome $\mathrm{X}: 19,533,975-19,887,601$ reverse strand) was chosen as this exon is present in almost all CIN85 isoforms (compare ENSG00000147010). The TAL Effector Nucleotide Targeter 2.0 software from the Cornell University was used to design the constructs. Here, TALEN constructs were selected, which target sites overlapping with the binding site of the BpuEI restriction enzyme to enable convenient testing of successfully targeted genomic DNA.

After nucleofection with the two TALEN constructs and subsequent cell sorting for GFP and RFP positive cells, genomic DNA was isolated and the region of exon 4 of the CIN85 gene was amplified. Cleavage with the restriction enzyme BpuEl (NEB) that binds at the cutting site of the TALEN constructs revealed activity of the generated TALEN constructs, because BpuEl did not cut as efficient the exon 4 region amplicon of TALEN-treated compared to control cells (figure 3.2.A). After subcloning, cleared cellular lysates were prepared and subjected to SDS-PAGE. Immunoblotting showed a high number of CIN85deficient DG75 cell clones (CIN85 $)$. In this thesis, I will focus on the three clones \#31, \#34 and \#70 (figure 3.2.B). Sequencing of exon 4 was performed to prove altered DNA 
sequences in this targeted exon. As an example, sequence analysis of clone \#34 is shown. It had a 108 bp deletion that also affected the previous intron region (figure 3.2.C). Therefore, it is very unlikely that exon 4 can be transcribed because the intron 3-exon 4 splicing site is located in the deleted region, preventing CIN85 protein translation. Clone \#34 was used for the following experiments as representative.
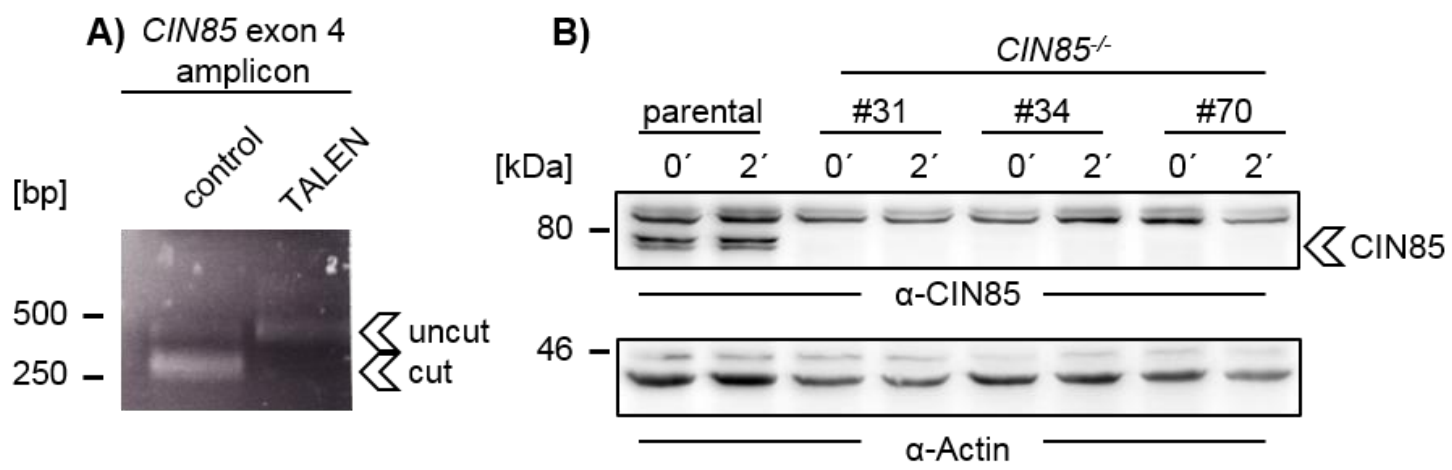

C) parental allele AAGAGCTGTC ...100bp... AGTTGGCGAC ATCATAGAGG CIN85 $\%$ \#34 AAGAGCTG-- ...100bp....-----CGAC ATCATAGAGG

D)

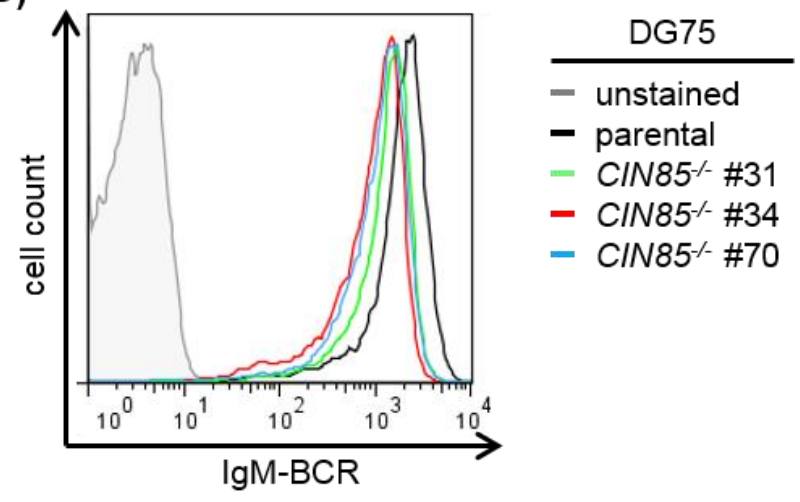

Figure 3.2: TALEN-mediated loss of CIN85 in DG75 B cells. A) To test the activity of the TALEN constructs, the targeted exon 4 region of CIN85 from TALEN-treated and control cells was amplified by PCR and a BpuEI restriction was performed. The BpuEl binding site overlaps with the TALEN cutting site and hence allows for activity test of the TALEN constructs. B) Cleared cellular lysates of DG75 parental and putative CIN85-deficient clones \#31, 34 and 70 were subjected to SDS-PAGE and the immunoblot was probed with anti-CIN85 and anti-Actin antibodies. The respective molecular weight of marker proteins (in kDa) is indicated on the left. C) Sequencing of the CIN85 exon 4 amplicon of CIN85 $\# 34$ compared to the parental allele. D) Representative flow cytometric analysis of surface BCR with an anti-hlgM-CY5 antibody.

Since the amount of surface BCR determines signal strength (Rowland et al., 2010) I tested for IgM expression on CIN85-deficient DG75 cells. The results showed that surface 
expression of IgM-BCR was similar in the analyzed clones and parental cells so that they could be used in further studies.

3.2.2 Tyrosine phosphorylation events upon BCR stimulation are not affected by the loss of CIN85 expression

Tyrosine phosphorylation of proteins involved in the BCR signaling cascade is an important readout for proper signal transduction, since it mediates complex formation and activation of BCR signaling effectors. To test whether the lack of CIN85 expression influences the overall tyrosine phosphorylation kinetics of BCR downstream components, cleared cellular lysates were subjected to SDS-PAGE and immunoblots were stained for phosphorylated tyrosine (figure 3.3.A). The signals of tyrosine phosphorylated proteins upon BCR stimulation between the parental cells and the analyzed CIN85-deficient clones were similar. The same applied for a detailed look on phosphorylation of the downstream kinases Akt and Erk of BCR signaling in figure 3.3.B. This indicates that CIN85 is not a major mediator in BCR-induced phosphorylation events. Nevertheless, impact on individual proteins cannot be excluded based on these data.
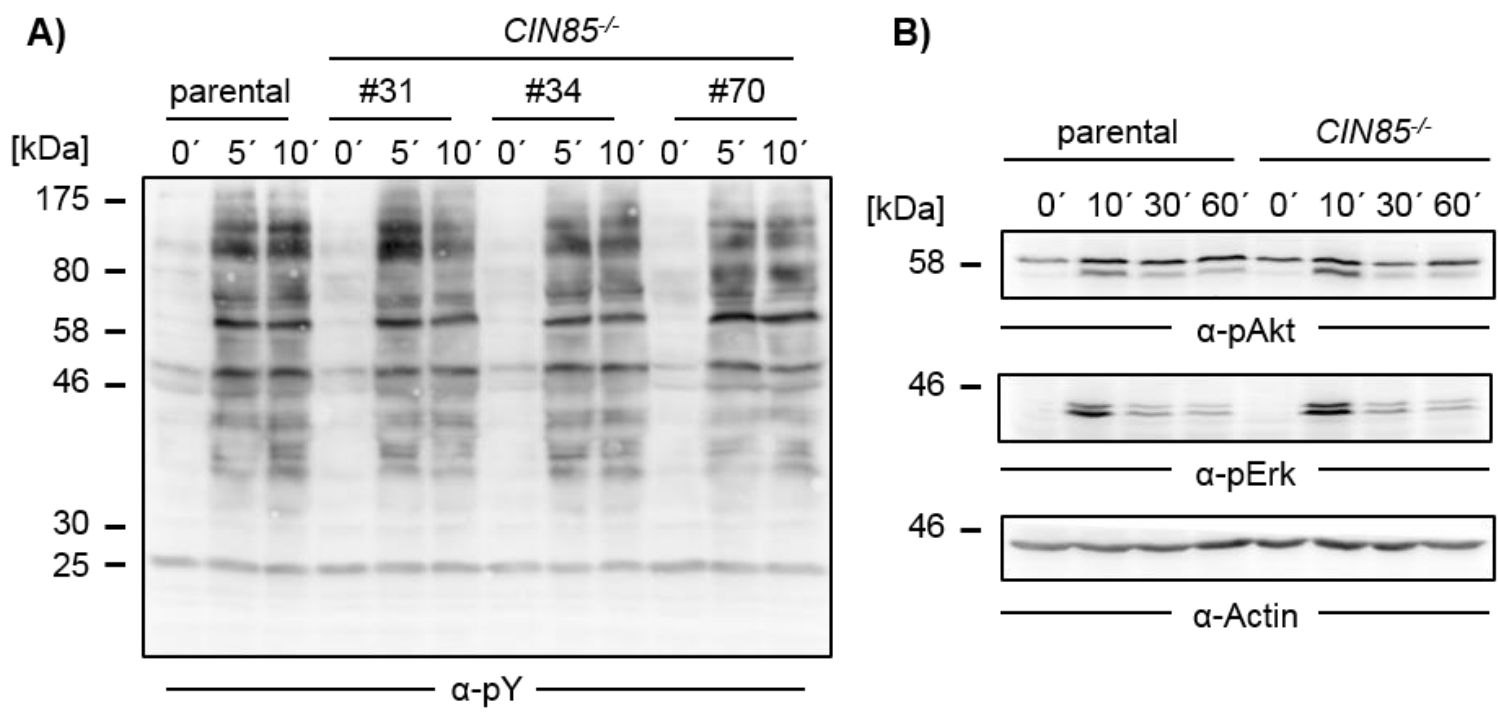

Figure 3.3: Loss of CIN85 does not influence BCR-mediated tyrosine phosphorylation events. A) DG75 parental and the CIN85-deficient cell clones \#31, \#34 and \#70 were left untreated $\left(0^{\prime}\right)$ or stimulated with 10 $\mu \mathrm{g} / \mathrm{ml} \alpha-\operatorname{lgM~} F\left(a b^{\prime}\right)_{2}$ fragments for $5\left(5^{\prime}\right)$ and $10 \mathrm{~min}\left(10^{\prime}\right)$. Cleared cellular lysates were subjected to SDSPAGE and the immunoblot was stained with anti-phospho tyrosine (pY) antibodies. B) Immunoblot of CCL of untreated $\left(0^{\prime}\right), 10 \mathrm{~min}\left(10^{\prime}\right), 30 \mathrm{~min}\left(30^{\prime}\right)$ and $60 \mathrm{~min}\left(60^{\prime}\right)$ stimulated DG75 parental and CIN85 $\# 34$ cells with $10 \mu \mathrm{g} / \mathrm{ml}$ anti-lgM $\mathrm{F}\left(\mathrm{ab}^{\prime}\right)_{2}$ fragments was stained with anti-phospho Akt, anti-phospho Erk and anti-Actin. The respective molecular weight of marker proteins (in $\mathrm{kDa}$ ) is indicated on the left. 


\subsubsection{Loss of CIN85 moderately impairs BCR-mediated $\mathrm{Ca}^{2+}$ signaling}

$B$ cells of the CIN85-deficient patient showed decreased $\mathrm{Ca}^{2+}$ mobilization upon stimulation with anti-lgM $\mathrm{F}\left(\mathrm{ab}^{\prime}\right)_{2}$ fragments compared to a healthy control (unpublished data). The mobilization of $\mathrm{Ca}^{2+}$ is an important step in BCR-mediated $\mathrm{B}$ cell activation in which the constitutive interaction partner of CIN85, SLP65, plays an essential role (Chiu et al., 2002). The impact of the preformed complex consisting of CIN85 and SLP65 on BCRinduced $\mathrm{Ca}^{2+}$ mobilization has been shown in the DT40 cell system. Here, shRNA mediated decrease of CIN85 protein expression in DT40 cells diminished the BCRinduced $\mathrm{Ca}^{2+}$ response when physiological amounts of stimulating antibodies were used for crosslinking of surface $\operatorname{lgM}$ (Bremes, 2012). To analyze the role of CIN85 deficiency on $\mathrm{Ca}^{2+}$ mobilization in human $\mathrm{B}$ cells, representative CIN85-deficient cell clones were compared to parental DG75 cells. Stimulation with $10 \mu \mathrm{g} / \mathrm{ml}$ anti-lgM $\mathrm{F}\left(\mathrm{ab}^{\prime}\right)_{2}$ fragments induced a similar $\mathrm{Ca}^{2+}$ mobilization profile in all analyzed samples. Nevertheless, the CIN85-deficient cell clones \#31 and \#34 showed reduced $\mathrm{Ca}^{2+}$ influx into the cytoplasm, whereas clone \#70 showed no difference compared to the parental cells (figure 3.4.A). However, stimulation with low amounts of anti-lgM $F\left(a b^{\prime}\right)_{2}$ fragments $(1 \mu \mathrm{g} / \mathrm{ml})$ that resemble more the physiological situation led to a lower $\mathrm{Ca}^{2+}$ response of all CIN85deficient clones compared to parental cells (figure 3.4.B). This was also true for other tested CIN85 $5^{\prime}$ cell clones (data not shown). To rule out unwanted side effects by TALEN gene targeting, CIN85-deficient DG75 cells were retrovirally transduced with a construct, which encodes for citrine-tagged wild-type CIN85 (CIN85wt). As a negative control a construct coding for EGFP was transduced (figure 3.4.C). $\mathrm{Ca}^{2+}$ signal in the CIN85 reconstituted cells resembled that of the parental cells (figure 3.4.D) implying that the reduction in mobilized $\mathrm{Ca}^{2+}$ of CIN85-deficient DG75 cells was not due to a clonal effect but founded on the lack of CIN85 protein. This confirmed the results obtained in the chicken B cell line DT40 and importantly the phenotype of the patient. Thus the generated CIN85 DG75 sub-line is an appropriate model system to elucidate the patients' phenotype further. 
A)

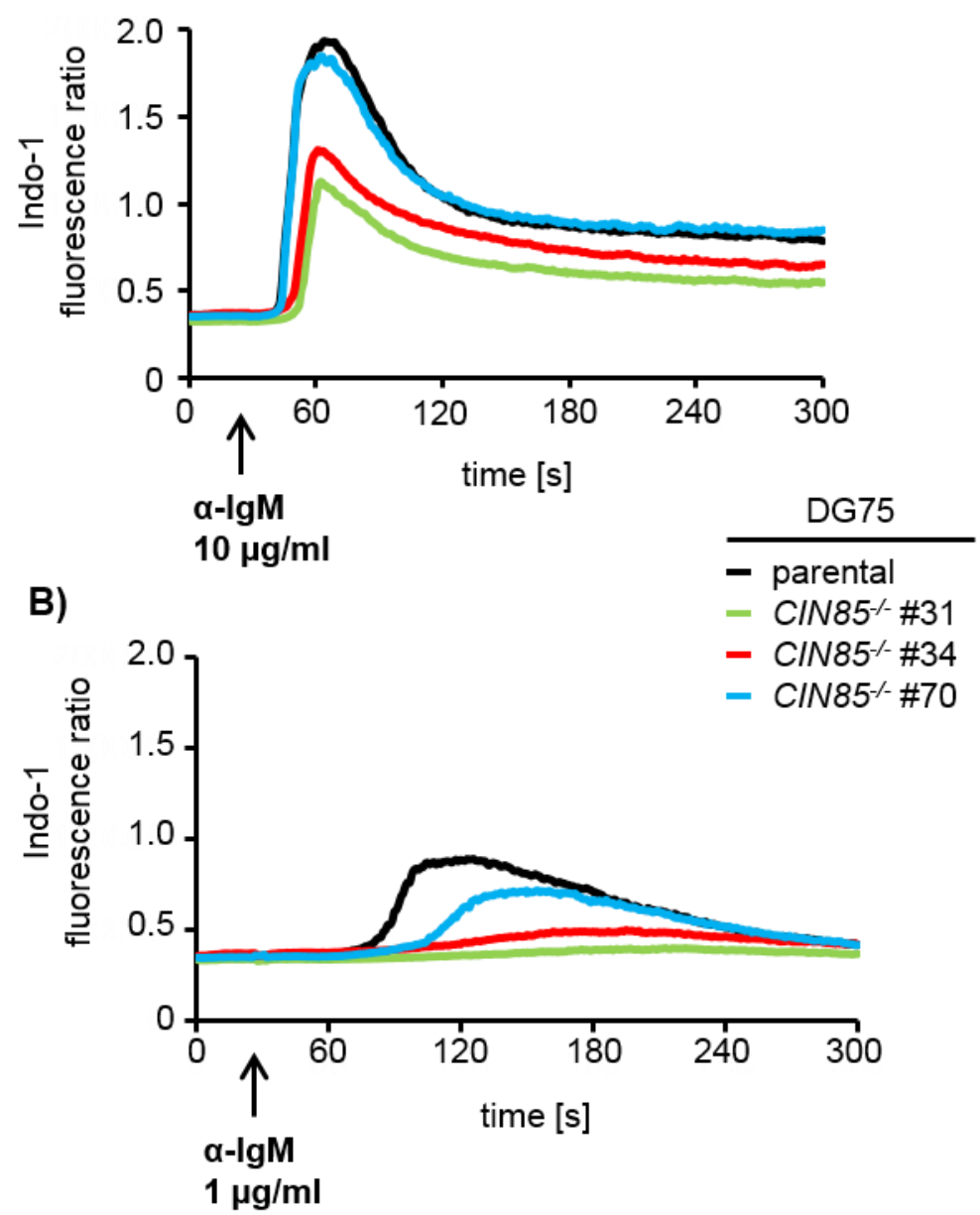

C)

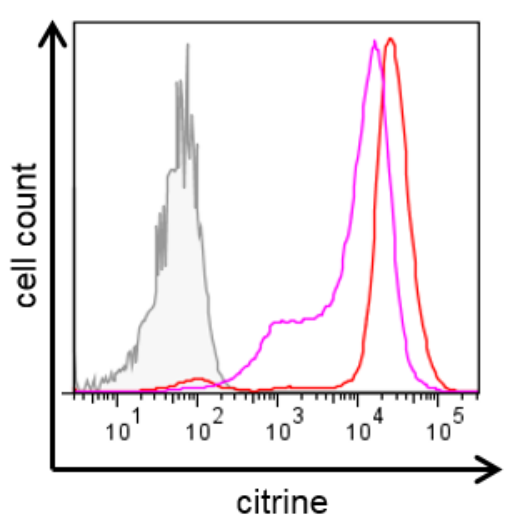

D)

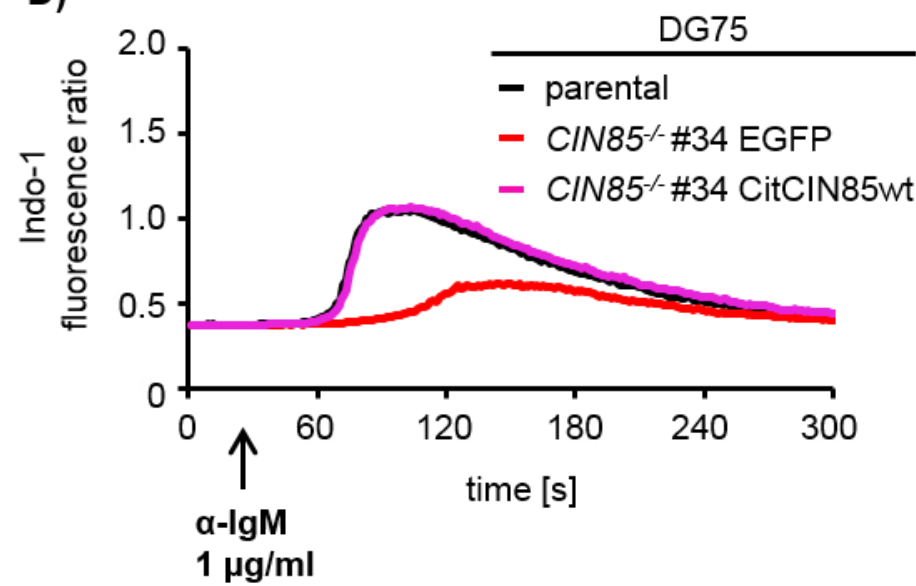

Figure 3.4: CIN85 deficiency leads to reduced $\mathrm{Ca}^{2+}$ signaling upon $\mathrm{BCR}$ activation. A) and $\left.\mathrm{B}\right) \mathrm{Ca}^{2+}$ measurement of DG75 parental (black), CIN85 $\# 31$ (green), \#34 (red) and \#70 (blue) cells. Cells were loaded with the $\mathrm{Ca}^{2+}$ sensitive dye Indo1-AM in order to measure intracellular $\mathrm{Ca}^{2+}$-mobilization by flow cytometry. After base line recording for $20 \mathrm{~s}$, cells were stimulated with $10 \mu \mathrm{g} / \mathrm{ml}$ (A) and $1 \mu \mathrm{g} / \mathrm{ml}$ (B) antihuman $\lg M F\left(a b^{\prime}\right)_{2}$ fragments $(\alpha-\lg M)$, respectively as indicated by an arrow. C) Representative flow cytometric analysis of DG75 C/N85 ${ }^{-}$retrovirally transduced with constructs coding for citrine-tagged wild-type CIN85 (CitCIN85wt) or EGFP (color code in D)). D) Cells depicted in C) were analyzed for $\mathrm{Ca}^{2+}$ mobilization as in $A)$ with $1 \mu \mathrm{g} / \mathrm{ml}$ anti-lgM $F\left(a b^{\prime}\right)_{2}$ fragments. 
3.2.4 Both SH3 and coiled coil domains are mandatory for membrane recruitment of CIN85 and $\mathrm{Ca}^{2+}$ signaling

Efficient $\mathrm{BCR}$-induced $\mathrm{Ca}^{2+}$ mobilization is conditioned by recruitment of signaling proteins to the plasma membrane. As CIN85 influenced the $\mathrm{Ca}^{2+}$ response, its membrane translocation upon BCR engagement was exerted. Therefore, CIN85-deficient DG75 cells expressing citrine-tagged wild-type CIN85 (CIN85wt) (figure 3.5.A) were analyzed by confocal laser scanning microscopy. In resting cells, CIN85 was located in the cytosol and upon BCR stimulation it was recruited to the plasma membrane (figure 3.5.B).

A)

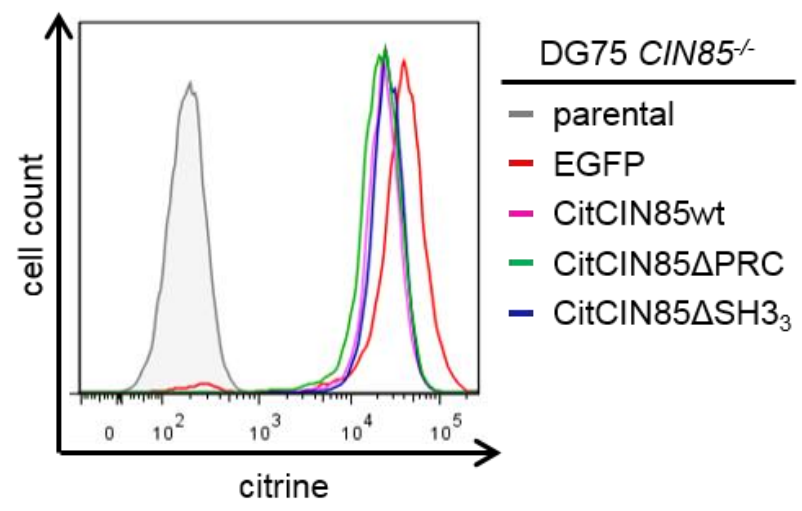

C)

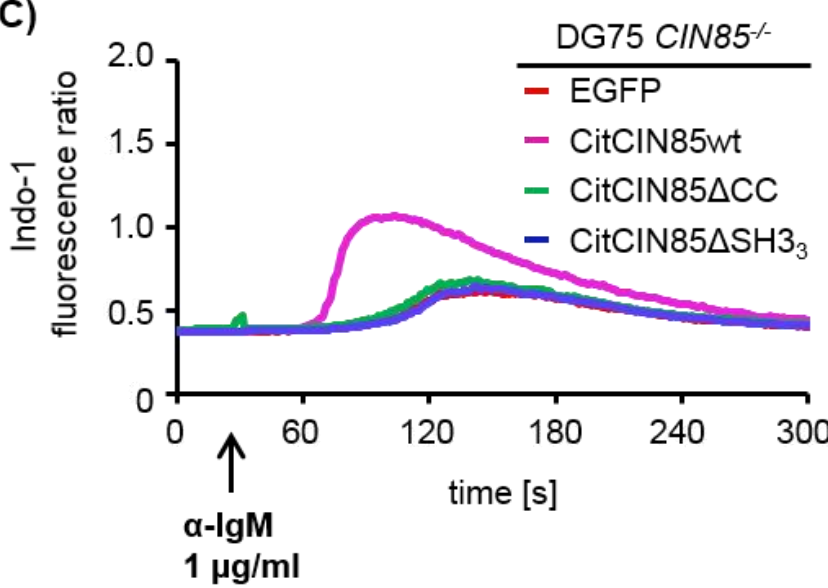

B)
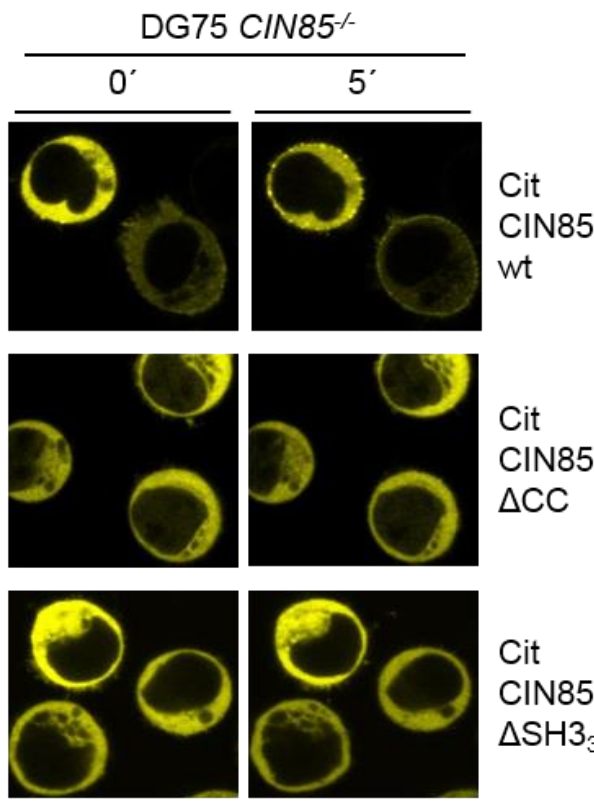

Cit CIN85 $\triangle \mathrm{SH}_{3}$

Figure 3.5: Truncated CIN85 variants are not sufficient to enable complete BCR signaling. A) DG75

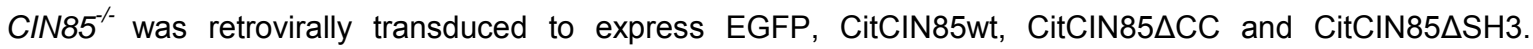
Analyses of expressed proteins was done by flow cytometry. B) Cells described in A) were subjected to confocal laser scanning microscopy and left untreated (left panel) or stimulated with $10 \mu \mathrm{g} / \mathrm{ml}$ anti- $\operatorname{lgM~F}\left(a b^{\prime}\right)_{2}$ fragment. C) Cells described in A) were measured for $\mathrm{Ca}^{2+}$ mobilization upon stimulation with $1 \mu \mathrm{g} / \mathrm{ml}$ anti$\mathrm{BCR}$ as before. 
To test, which domains of CIN85 are responsible for membrane translocation, the impact of the N-terminal $\mathrm{SH} 3$ and the C-terminal coiled coil (CC) domains were assessed. Therefore, CIN85-deficient cells were retrovirally transduced with constructs coding for citrine-tagged CIN85 truncation variants lacking either the three $\mathrm{SH} 3$ domains $\left(\Delta \mathrm{SH}_{3}\right)$ or the $\mathrm{CC}$ domain $(\triangle \mathrm{CC})$. Expression of the citrine-tagged proteins was proven with flow cytometry (figure 3.5.A) and cellular localization was analyzed. Both variants were located in the cytoplasm in resting cells, but neither CIN85 $\Delta \mathrm{CC}$ nor $\mathrm{CIN} 85 \Delta \mathrm{SH} 3_{3}$ could be recruited to the plasma membrane upon BCR stimulation (figure 3.5.B). These experiments showed that both the $\mathrm{SH} 3$ as well as the $\mathrm{CC}$ domains are essential for plasma membrane recruitment of CIN85.

Furthermore, I analyzed the $\mathrm{Ca}^{2+}$ mobilization in cells expressing one of these truncated CIN85 variants. Upon BCR stimulation with low antibody concentrations, cells expressing the truncated CIN85 variants showed an identical $\mathrm{Ca}^{2+}$ profile similar to the EGFP expressing cells (figure 3.5.C). Supporting plasma membrane recruitment results, neither the $\mathrm{SH} 3$ domains nor the $\mathrm{CC}$ domain alone were sufficient to fulfill CIN85 adaptor functions in BCR-induced $\mathrm{Ca}^{2+}$ mobilization, which indicates that only full length CIN85 is completely functional in plasma membrane translocation and $\mathrm{Ca}^{2+}$ mobilization.

\subsubsection{Membrane recruitment of CIN85 is not influenced by Lyn interaction}

I showed that CIN85 translocated to the plasma membrane upon BCR stimulation in human B cells but the membrane anchor for CIN85 is still unknown. Vanessa Bremes could show that CIN85 plasma membrane recruitment depends on the kinase function of Lyn in DT40 cells (Bremes, 2012), whereas Lyn is the only src kinase expressed in DT40 cells. As Lyn is furthermore reported to associate with its SH3 domain with CIN85 (Narita et al., 2005) interaction of CIN85 and Lyn in B cells was tested with affinity purification experiments in Lyn-deficient DT40 cells using GST-Lyn SH3 domain. As a control GSTLyn SH3P114L was used. The proline to leucine substitution in this construct mediated unfunctionality of the SH3 domain (Yokoyama et al., 2002) and prevented interaction of Lyn with CIN85 that appeared when the wild-type Lyn SH3 domain was used instead (figure 3.6.A). For elucidating whether this interaction mediated CIN85 plasma membrane recruitment Lyn-deficient DT40 cells expressing either HA-tagged wild-type Lyn (DT40LYN ${ }^{-}$Lynwt) or HA-tagged Lyn P114L (DT40LYN LynP114L) variants (M. Lösing) were retrovirally transduced to express citrine-tagged wild-type CIN85 (CitCIN85wt). For examination of CIN85 plasma membrane translocation dependent on Lyn SH3 interaction confocal laser scanning microscopy was performed (figure 3.6.B). CIN85 was located in the cytoplasm and upon BCR stimulation recruitment to the plasma membrane was 
induced in both Lynwt (upper panels) and LynP114L (lower panels) expressing cells. This indicated that direct interaction with the Lyn SH3 domain did not mediate CIN85 membrane recruitment. Hence, another mechanism than interaction with the plasma membrane resident Lyn kinase has to mediate plasma membrane recruitment of CIN85.

A)

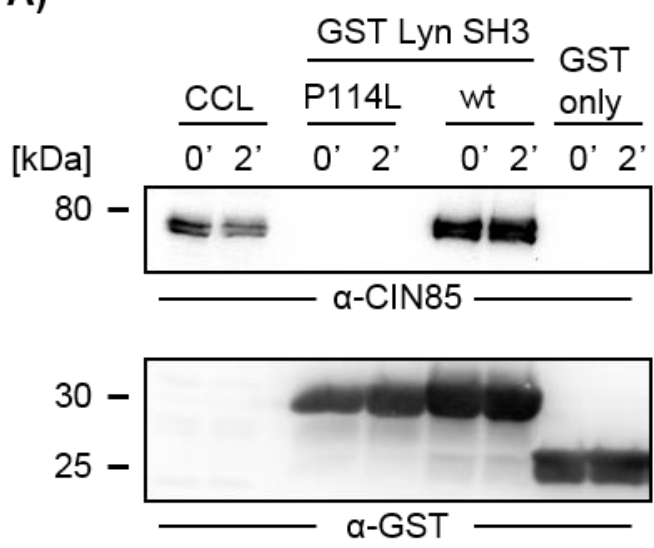

B)

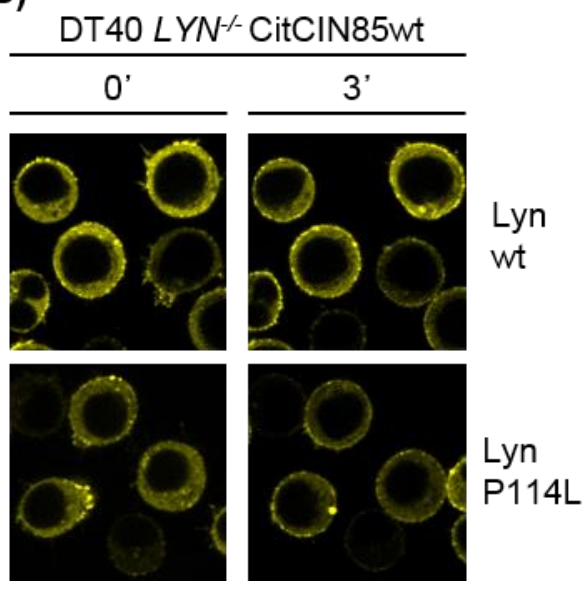

Figure 3.6: CIN85-Lyn interaction does not mediate CIN85 plasma membrane recruitment. A) Cleared cellular lysates from resting ( $\left.0^{\prime}\right)$ and 2 min stimulated (2') DT40 cells with $2 \mu \mathrm{g} / \mathrm{ml}$ anti-lgM (M4) were directly used for immunoblotting or for subsequent affinity purification with GST-Lyn SH3 domain wild-type (wt) and proline 114 to leucine (P114L) variants. GST-only was carried along. The respective molecular weight of marker proteins (in $\mathrm{kDa}$ ) is indicated on the left. B) DT40 $L Y N^{\wedge}$ HA-Lynwt and HA-LynP114L were retrovirally transduced with constructs coding for citrine-tagged wild-type CIN85 (DT40 LYN ${ }^{\wedge}$ CitCIN85wt Lynwt, DT40 $L Y N^{\prime-}$ CitCIN85wt LynP114L). The cells were subjected to confocal laser scanning microscopy and left unstimulated (left panel) or treated with $2 \mu \mathrm{g} / \mathrm{ml}$ anti-IgM (M4) for $3 \mathrm{~min}\left(3^{\prime}\right)$ (right panel).

\subsubsection{CIN85 positively regulates the function of SLP65}

Since CIN85 constitutes an activating role in the BCR-induced $\mathrm{Ca}^{2+}$ response and it is known to be a steady ligand of the key adaptor SLP65, this interaction is likely to be a crucial point in that context. Experiments performed in chicken B cells did show that the interaction between CIN85 and SLP65 is of importance for BCR-induced signaling (Oellerich et al., 2011). To confirm this in the human B cell line DG75, a variant of SLP65 was used, which neither can bind to CIN85 nor its homolog CD2AP. Therefore, arginine $(R)$ to alanine $(A)$ substitutions at position 248 and 313 were introduced into the atypical proline-arginine motifs 2 and 3 of SLP65 (SLP65M23) that mediate the interaction with the CIN85 SH3 domains. The M23 variant of SLP65 was N-terminally tagged with citrine (CitSLP65M23) and expressed in SLP65-deficient DG75 cells (kindly provided by Christoffer Hitzing). Expression of analyzed proteins was tested with flow cytometry (figure 3.7.A). The $\mathrm{Ca}^{2+}$ profile of the CIN85/CD2AP interaction deficient SLP65 M23 variant 
expressing cells was reduced compared to the parental cells and resembled that of the CIN85-deficient cells (figure 3.7.B). This validates that the interaction of SLP65 with CIN85/CD2AP enhances $\mathrm{Ca}^{2+}$ signaling upon BCR stimulation also in human DG75 B cells.

A)

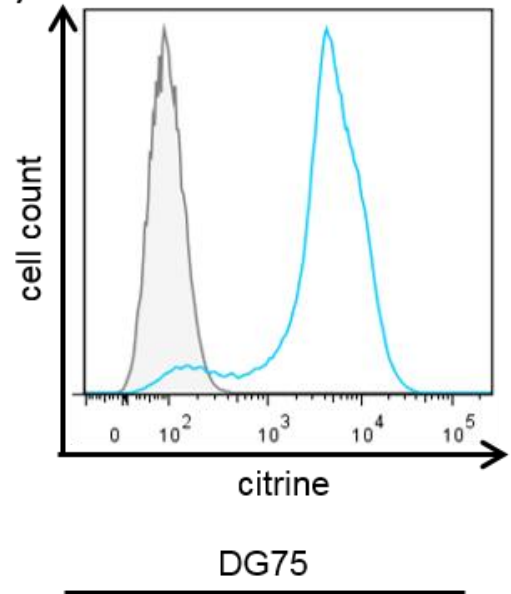

- parental

- SLP65\%CitSLP65M23
B)

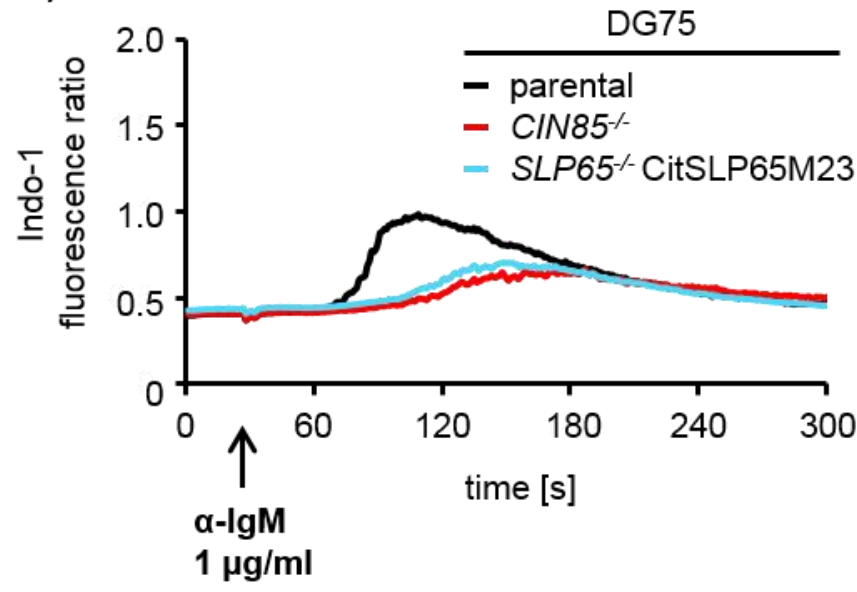

C)

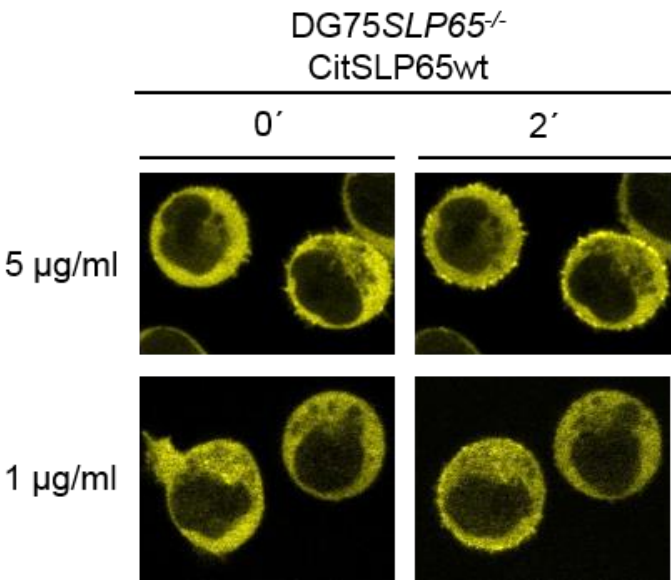

D)

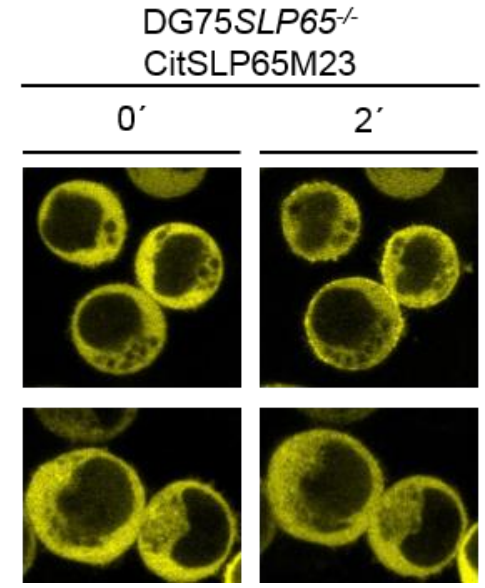

Figure 3.7: CIN85 interaction with SLP65 positively modulates SLP65 function in $\mathrm{Ca}^{2+}$ flux and plasma membrane recruitment in human B cells. A) SLP65-deficient cells were retrovirally transduced with constructs encoding citrine-tagged SLP65 M23 (CitSLP65M23). The expression was determined by flow cytometry. B) The cells described in A) were analyzed together with parental and $\mathrm{CIN} 5^{/ .}$for $\mathrm{Ca}^{2+}$ mobilization upon stimulation with $1 \mu \mathrm{g} / \mathrm{ml} \mathrm{F}\left(\mathrm{ab}^{\prime}\right)_{2}$ fragments as before. C) SLP65-deficient DG75 cells were retrovirally transduced with citrine-tagged wild-type SLP65 (CitSLP65wt) or CitSLP65M23 (D) and analyzed by confocal laser scanning microscopy for BCR-mediated plasma membrane recruitment of SLP65 after stimulation with 5 $\mu \mathrm{g} / \mathrm{ml}$ (upper panels) or $1 \mu \mathrm{g} / \mathrm{ml}$ (lower panels, respectively) anti-lgM $\mathrm{F}\left(\mathrm{ab}^{\prime}\right)_{2}$ fragments. Images were taken before and 2 imn after BCR stimulation.

In addition, the influence of CIN85 and CD2AP on SLP65 membrane recruitment was analyzed by confocal laser scanning microscopy. Citrine-tagged wild-type SLP65 
(SLP65wt) was located in the cytoplasm and rapidly recruited to the plasma membrane upon BCR stimulation with $5 \mu \mathrm{g} / \mathrm{ml}$ anti-IgM $\mathrm{F}\left({ }^{\prime} \mathrm{ab}\right)_{2}$ fragments (figure 3.7.C). SLP65wt also translocated to the membrane when the amount of stimulating antibody was decreased to $1 \mu \mathrm{g} / \mathrm{ml}$ anti-lgM F('ab) ${ }_{2}$ fragments. The M23 variant was also recruited to the membrane after stimulation with $5 \mu \mathrm{g} / \mathrm{ml}$ anti-lgM $\mathrm{F}\left({ }^{\prime} \mathrm{ab}\right)_{2}$ fragments, but in contrast to SLP65wt it stayed in the cytoplasm when limited antibody concentrations were applied (figure 3.7.D).

In summary, the SLP65 M23 variant, which is incapable of binding to CIN85 and CD2AP, is dependent on BCR stimulation with high amounts of stimulating antibody for proper plasma membrane recruitment and $\mathrm{Ca}^{2+}$ signaling. Here, CIN85 operates as an amplifier for SLP65 function.

\subsection{CIN85 regulates the function of SLP65 in BCR-induced $\mathrm{Ca}^{2+}$ signaling}

\subsubsection{Generation of a TALEN-mediated CIN85/SLP65 double-deficient DG75} sub-line

As the SLP65 M23 variant does not only circumvent binding to CIN85 but also to its homolog CD2AP, the exclusive impact of CIN85 on SLP65 cannot be determined. In addition, it has to be noted that there might be further interactors of both proline-arginine motifs that influence SLP65 function in the here performed experimental systems.
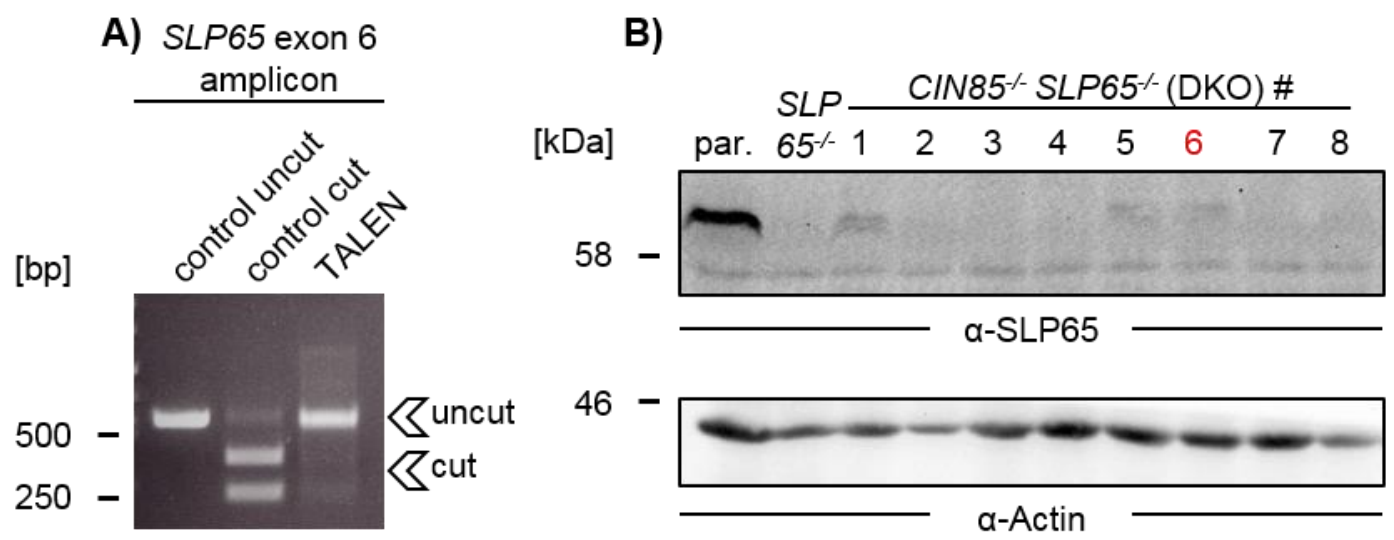

Figure 3.8: Generation of CIN85/SLP65 double-deficient DG75 cells. A) To test the activity of the TALEN constructs, the targeted exon 6 region of TALEN-treated CIN85-deficient DG75 cells and DG75 control cells was amplified by PCR and a Bmrl restriction was performed. The Bmrl binding site overlaps with the TALEN cutting site and hence allows for activity test of the TALEN constructs. B) Cleared cellular lysates of DG75 parental (par.), SLP65-deficient $\left(S L P 65^{\circ}\right)$ and DKO cell clones \#1, \#2, \#3, \#4, \#5, \#6, \#7 and \#8 were subjected to SDS-PAGE and the immunoblot was probed with anti-SLP65 and anti-Actin antibodies. Cell clone 
\#6 was used in the following experiments (marked in red). The respective molecular weight of marker proteins (in $\mathrm{kDa}$ ) is indicated on the left.

To investigate the relevance of the CIN85-SLP65 interaction for SLP65 function, a DG75 sub-line deficient for CIN85 and SLP65 was generated. Therefore, I applied the TALEN method to target SLP65 in the CIN85-deficient DG75 cell clone \#34. The TALEN constructs targeting exon 6 of the human SLP65 gene were kindly provided by Christoffer Hitzing. Transient transfection of TALEN constructs, cell sorting and subsequent activity test (figure 3.8.A) were performed as described in chapter 3.2.1. The cells were consecutively subcloned and cleared cellular lysates were prepared to test for loss of SLP65 expression. As shown in figure 3.8.B, efficiency of the TALEN constructs was very high as almost every tested cell clone lacked SLP65 expression. The CIN85/SLP65 double-deficient DG75 cell clone \#6 was used in all following experiments and is hereafter referred to as DKO.

To investigate whether the CIN85 enhancing function in $\mathrm{Ca}^{2+}$ signaling depends exclusively on the support of SLP65, I compared DKO DG75 cells with SLP65-deficient cells reconstituted with either SLP65wt or EGFP in $\mathrm{Ca}^{2+}$ mobilization experiments (figure 3.9.A). The DKO cells had a reduced $\mathrm{Ca}^{2+}$ signaling that matched the phenotype of the sole SLP65-deficient cells expressing EGFP. This indicates that SLP65 deficiency already mediates a severe phenotype in terms of $\mathrm{Ca}^{2+}$ mobilization, which is not further impaired by an additional loss of CIN85.

Next, reconstitution experiments were performed to prove that the observed effects for the DKO (figure 3.9.A) are based on the absence of CIN85 and SLP65 and are no unwanted site effects. $\mathrm{Ca}^{2+}$ mobilization experiments with these cells revealed that SLP65wt expressing DKO cells were capable of mediating more $\mathrm{Ca}^{2+}$ influx compared to DKO cells, but it was less compared to SLP65-deficient cells expressing SLP65wt (figure 3.9.B, C). In contrast, expression of CIN85wt in the DKO DG75 cells did not restore the diminished $\mathrm{Ca}^{2+}$ flux, confirming the supporting function of CIN85 for its constitutive binding partner SLP65 in this context. 
A)

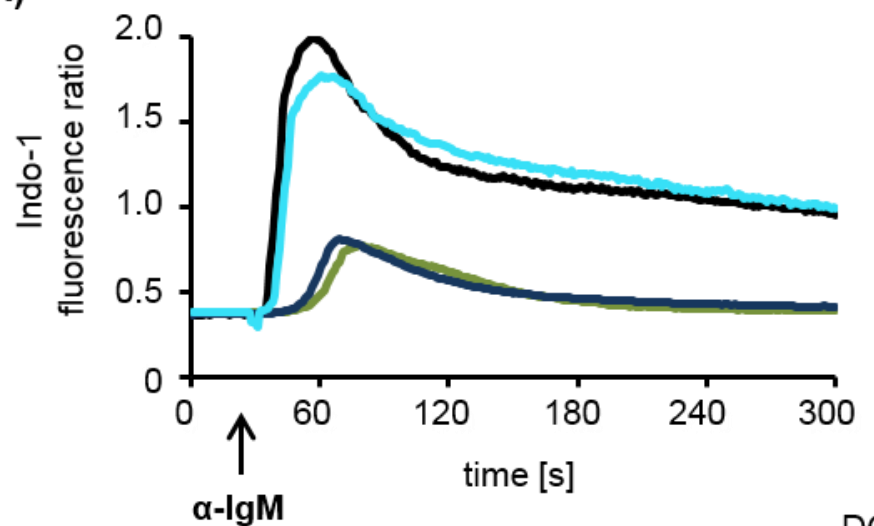

$10 \mu \mathrm{g} / \mathrm{ml}$

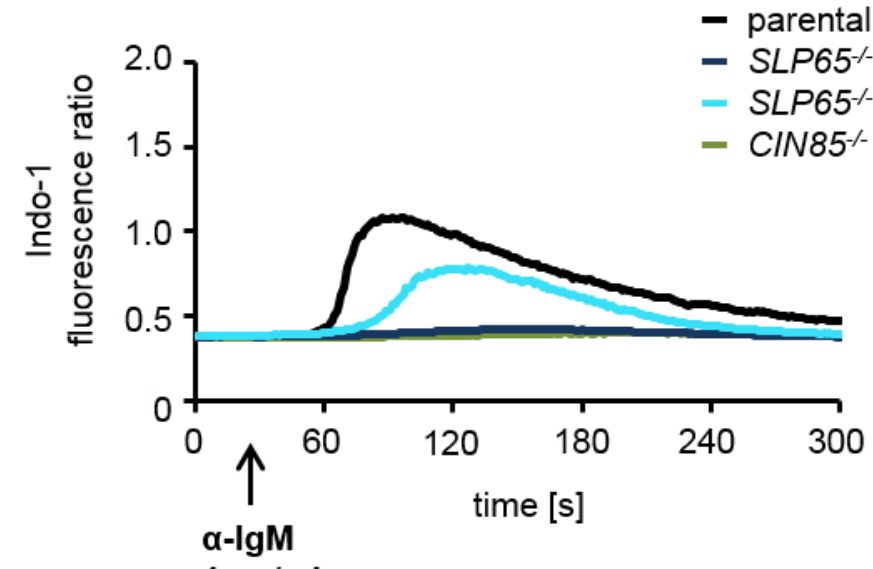

B)

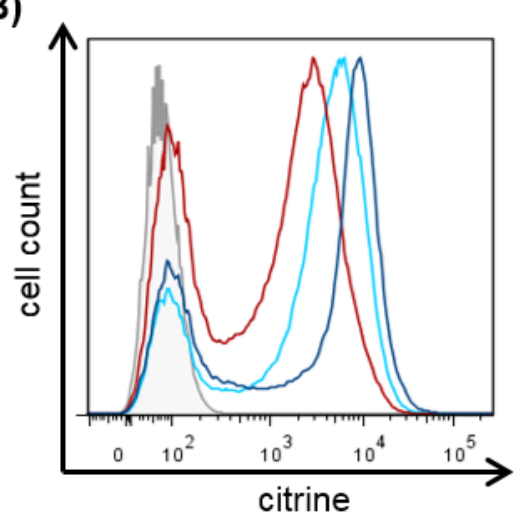

C)

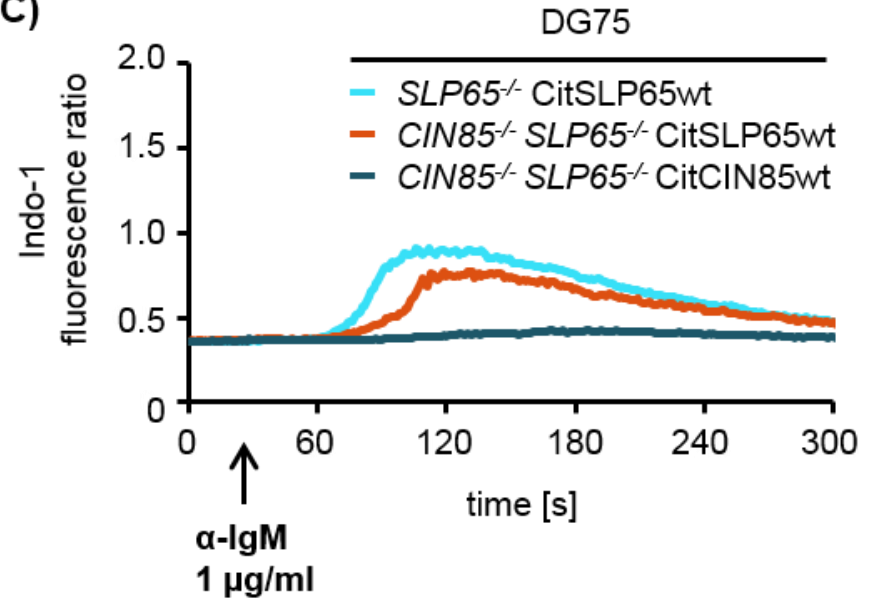

Figure 3.9: CIN85 and SLP65 act in concert to enhance BCR-induced $\mathrm{Ca}^{2+}$ mobilization. A) The DKO DG75 cell clone \#6 and SLP65-deficient DG75 cells expressing CitSLP65wt or EGFP were subjected to $\mathrm{Ca}^{2+}$ flux measurements upon BCR stimulation with $10 \mu \mathrm{g} / \mathrm{ml}$ (upper panel) and $1 \mu \mathrm{g} / \mathrm{ml}$ (lower panel) as before. B) The DKO cells were reconstituted with citrine-tagged wild-type SLP65 (CIN85 ${ }^{\circ}$ SLP65 ${ }^{-}$CitSLP65wt) or wildtype CIN85 (CIN85 ${ }^{-}$SLP65 $5^{-}$CitCIN85wt). Expression of analyzed proteins was tested by flow cytometry. C) $\mathrm{Ca}^{2+}$ mobilization measurement of DKO cells reconstituted with either CitSLP65wt or CitCIN85wt, and SLP65 ${ }^{-1-}$ CitSLP65wt cells after BCR crosslinking with $1 \mu \mathrm{g} / \mathrm{ml}$ of $\alpha$-lgM was done as before. 


\subsubsection{Plasma membrane recruitment of SLP65 is supported by CIN85}

I could show that CIN85 supports SLP65 function in the context of BCR-induced $\mathrm{Ca}^{2+}$ signaling in the human DG75 B cell line. Hence, I tested whether it also influences SLP65 membrane recruitment irrespective of CD2AP and other possible binding partners of both described proline-rich motifs (SLP65M23) in human DG75 cells. Therefore, reconstituted DKO cells with either SLP65wt or CIN85wt were used, which have the advantage that endogenous SLP65 and CIN85 proteins cannot interfere with the plasma membrane recruitment of ectopic expressed variants. At first, recruitment of citrine-tagged wild-type SLP65 expressed in either SLP65-deficient or DKO cells was compared. Citrine-tagged SLP65wt was rapidly ( $2 \mathrm{~min})$ translocated to the plasma membrane in SLP65-deficient DG75 cells like already shown in figure 3.7.C. In contrast, in DKO cells citrine-tagged SLP65 was recruited to the plasma membrane delayed in time ( $5 \mathrm{~min}$ ) (figure 3.10.B). Hence, CIN85 had an accelerating function in terms of SLP65 membrane recruitment.

A)

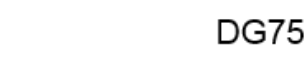

- parental

- SLP65 ${ }^{-1-}$ CitSLP65wt

- CIN85\% SLP65\% CitSLP65wt

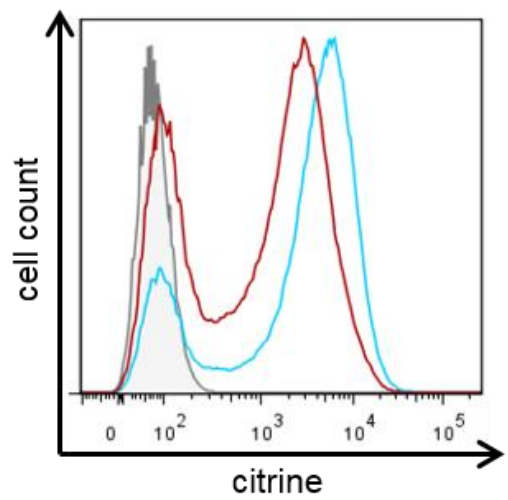

B)
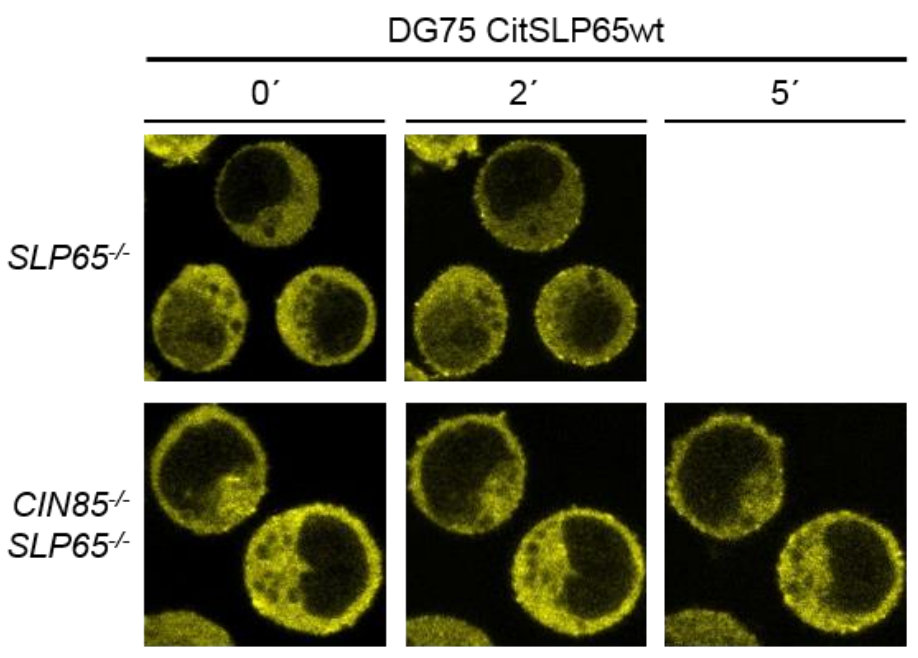

C)

DG75 CitCIN85wt

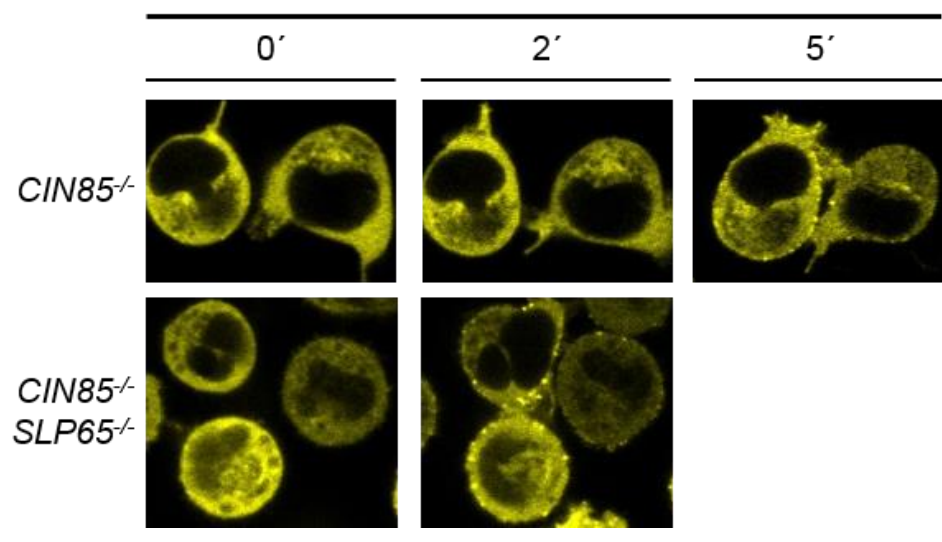


Figure 3.10: CIN85 enhances SLP65 plasma membrane recruitment. A) DG75 DKO and SLP65-deficient DG75 cells were retrovirally transduced with constructs encoding citrine-tagged wild-type SLP65 and the expression was tested by flow cytometry. B) The analyzed cells in A) were subjected to confocal laser scanning microscopy and images were taken before as well as $2 \min \left(2^{\prime}\right)$ and $5 \min \left(5^{\prime}\right)$ after stimulation with $5 \mu \mathrm{g} / \mathrm{ml}$ anti-IgM F(ab') $)_{2}$ fragments. C) CIN85-deficient DG75 and DG75 DKO cells expressing CitCIN85wt were analyzed as described in B) with the difference that stimulation was induced with $2 \mu \mathrm{g} / \mathrm{ml}$ anti-lgM $F\left(a b^{\prime}\right)_{2}$ fragments.

Vice versa, the impact of SLP65 on CIN85 plasma membrane recruitment was analyzed in DG75 DKO cells and compared to CIN85-deficient DG75 cells in figure 3.9.C. BCR stimulation induced rapid CIN85 plasma membrane recruitment with high amounts of antibody $(10 \mu \mathrm{g} / \mathrm{ml})$ in CIN85-deficient DG75 cells (compare figure 3.5.B). When stimulation was induced with reduced amounts of antibody $(2 \mu \mathrm{g} / \mathrm{ml})$, CIN85 was still recruited to the plasma membrane but delayed in time (figure 3.10.C). This decelerating effect was equalized when SLP65 was missing as in DKO cells expressing CIN85wt. Here, CIN85 recruitment was visible shortly after BCR stimulation.

Taken together, CIN85 is a positive regulator ofSLP65 membrane recruitment whereas the presence of SLP65 seems to slow CIN85 recruitment down.

\subsection{CIN85 mediates SLP65 protein stability}

\subsubsection{SLP65 protein expression is reduced in CIN85-deficient DG75 cells}

Protein levels in cells are kept in balance between newly synthesized and degraded proteins. Analysis of the CIN85-deficient DG75 sub-line showed reduced SLP65 protein levels compared to the parental cells (figure 3.11.A). To test whether this is based on clonal effects or a matter of loss of CIN85, cleared cellular lysates of parental, CIN85deficient and CIN85-deficient cells expressing CitCIN85wt were prepared. The immunoblot was stained for SLP65, CIN85 and Actin (figure 3.11.A). Reconstitution with CIN85 restored SLP65 protein expression so that SLP65 protein levels depend on the presence of CIN85.

As CIN85 interaction with SLP65 is mediated by the three $\mathrm{N}$-terminal SH3 domains of CIN85, it was tested whether these domains are competent to restore SLP65 expression. Therefore, cleared cellular lysates of CIN85-deficient DG75 cells expressing EGFP, CitCIN85wt, CitCIN85 $\triangle \mathrm{CC}$ or CitCIN85 $\mathrm{SH}_{3}$ were analyzed by immunoblotting and subsequent SLP65 staining (figure 3.11.B). As proof of expression of equal citrine-tagged CIN85 variants compare figure 3.5.A. CIN85 $\triangle$ CC restored the depleted SLP65 protein expression, whereas $\mathrm{CIN} 85 \Delta \mathrm{SH}_{3}$ did not. This indicates that direct interaction between 
the CIN85 SH3 domains and SLP65 influences SLP65 protein levels irrespective of the CIN85 CC domain.

A)

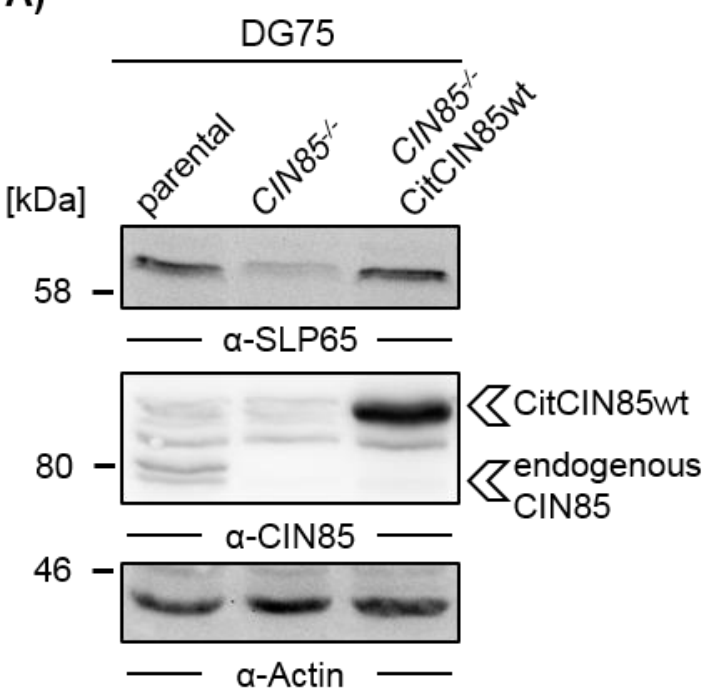

B)

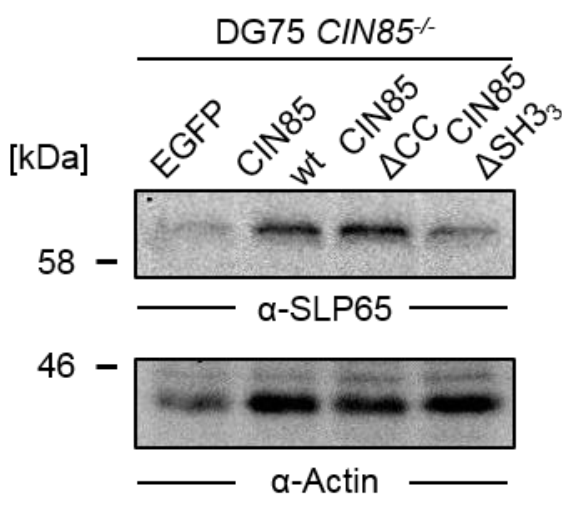

Figure 3.11: SLP65 expression is diminished in CIN85-deficient DG75 cells. A) Cleared cellular lysates of DG75 parental, CIN85-deficient and CIN85-deficient citrine-tagged wild-type CIN85 (CIN85/- CitCIN85wt) expressing cells were prepared, subjected to immunoblot and stained for SLP65, CIN85 and Actin as loading control. B) Cleared cellular lysates of CIN85-deficient DG75 cells expressing EGFP, CitCIN85wt, CitCIN85 $\triangle \mathrm{CC}$ or CitCIN85 $\triangle \mathrm{SH}_{3}$ were probed with anti-SLP65 and anti-Actin antibodies. The respective molecular weight of marker proteins (in $\mathrm{kDa}$ ) is indicated on the left.

Given that also CD2AP interacts via its SH3 domains with SLP65, shRNA-mediated knock down of CD2AP was applied to analyze its contribution on SLP65 protein expression. Reduction in CD2AP protein expression did not affect SLP65 protein (data not shown) and hence indicates that specifically CIN85 has to interact with SLP65 to rescue SLP65's protein amount in the cell.

\subsubsection{Protein degradation of SLP65 is prevented by CIN85}

To elucidate at what stage CIN85 affects SLP65 protein expression, SLP65 mRNA levels were analyzed. Therefore, mRNA of CIN85-deficient DG75 and CIN85-deficient DG75 cells reconstituted with CIN85wt was isolated, retranscribed into cDNA and used for quantitative real time PCR (qRT-PCR) analyses. In relation to the transcript levels of GAPDH, which served as housekeeping gene, the amount of SLP65 transcript was not altered in the CIN85-deficient cells compared with the reconstituted cells (figure 3.12.A). This demonstrates that CIN85 influences SLP65 expression not at the mRNA stage. 
Furthermore, the impact of CIN85 on SLP65 stability at protein level was assessed using the translation inhibitor cycloheximide (CHX) in CIN85-deficient and parental DG75 cells for different time periods. Cleared cellular lysates were prepared and the immunoblot was stained for SLP65 and both Syk and Actin were included as controls (figure 3.12.B). SLP65 had a rapid turnover in the parental cells as it was almost completely absent after $24 \mathrm{~h}$, if protein synthesis was blocked. In contrast, the control proteins were mostly unaffected. As the CIN85-deficient DG75 cells had already reduced amounts of SLP65 in the untreated state, protein was yet undetectable after $3 \mathrm{~h}$ of $\mathrm{CHX}$ treatment. Furthermore, the proteasome inhibitor MG132 was used to prevent ubiquitin-mediated degradation of SLP65. Therefore, parental and CIN85-deficient DG75 cells were treated for $10 \mathrm{~h}$ with either CHX or MG132 alone or with a combination of both. As negative control, DMSO was applied. Parental cells treated with $\mathrm{CHX}$ for $10 \mathrm{~h}$ had diminished SLP65 protein levels (figure 3.12.C, D). MG132 induced accumulation of SLP65 over basal level, since new protein can be translated. But when both reagents were applied, SLP65 levels were under basal level but above $\mathrm{CHX}$ only treatment. This indicates that the ubiquitin-proteasom pathway is not the only one that mediates SLP65 turnover.

A)

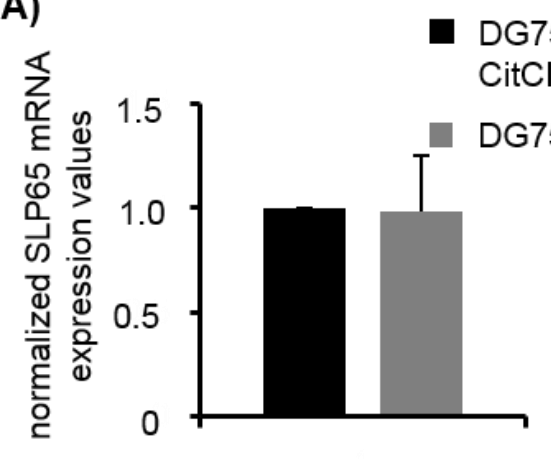

C)
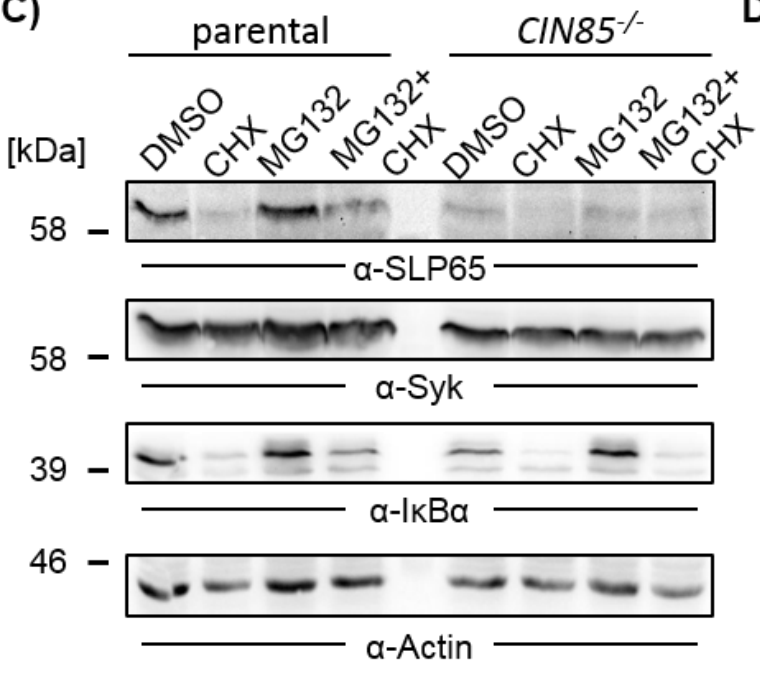

B)

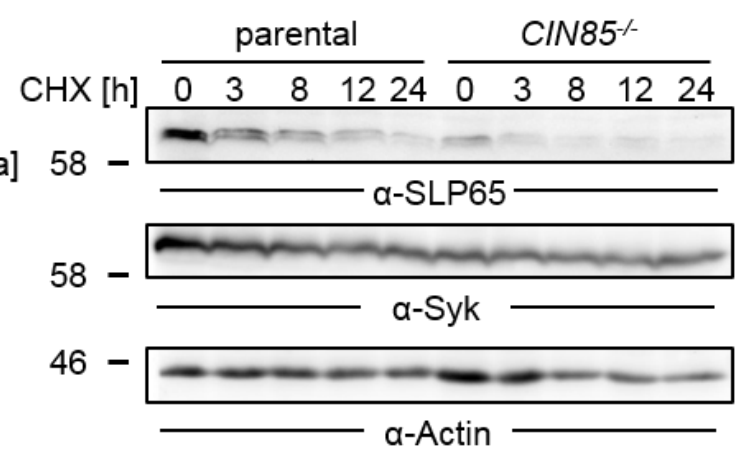

D)

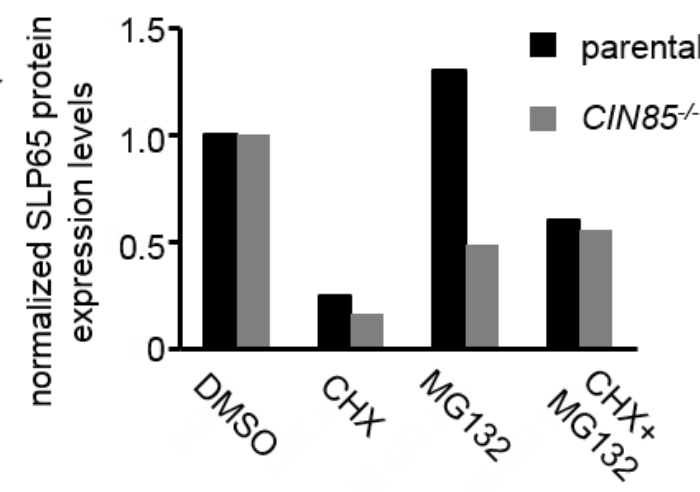


Figure 3.12: CIN85 stabilizes SLP65 protein levels. A) mRNA from CIN85-deficient DG75 (grey) and CIN85-deficient DG75 CitCIN85wt (black) cells was isolated and SLP65 expression was determined by qRTPCR. Data are depicted as mean of three independent experiments. B) DG75 parental and CIN85-deficient cells were left untreated (DMSO only) or treated with $25 \mu \mathrm{g} / \mathrm{ml} \mathrm{CHX}$ for 3, 8, 12 and 24 hours (h). Cleared cellular lysates were prepared and the immunoblot was stained for SLP65, or Syk and Actin as controls. C) DG75 parental and CIN85-deficient cells were either left untreated (DMSO only) (lane 1 and 5) or treated with $25 \mu \mathrm{g} / \mathrm{ml} \mathrm{CHX} \mathrm{(lane} 2$ and 6), $20 \mu \mathrm{M}$ MG132 (lane 3 and 7) or the combination of both (lane 4 and 8) for 10 hours each. Immunoblot of cleared cellular lysates was stained with anti-SLP65, anti-Syk, anti-IKBa or antiActin antibodies. D) Quantification of the SLP65 signal strength of figure C) related to Actin as loading control. Resulting relative SLP65 amounts of the different treatments were normalized to DMSO only.

CIN85-deficient DG75 cells solely treated with CHX showed an even more severe reduction of SLP65 protein in relation to the DMSO treated sample compared to the parental cells (figure 3.12.C, D). Exclusive treatment with MG132 or the combination of MG132 and CHX had the same outcome of protein amount in the cells.

As a protein that is known to be degraded by the ubiquitin-proteasome and the calpain pathway (Chen et al., 1995; Zhang et al., 1998; Han et al., 1999), IKBa was included in the experiment. This protein has a high turnover that is mediated by its PEST sequence (Shumway et al., 1999). Staining of the immunoblot with anti-IKBa antibodies revealed a correlating expression pattern with SLP65. Although the ubiquitin-proteasom-mediated pathway is not the only one that applies for $1 \kappa B \alpha$, there is accumulation with MG132 treatment. The difference compared to SLP65 is that IKBa also aggregates in CIN85deficient DG75 cells. This indicates that for SLP65 turnover another way of degradation next to the here tested ubiquitin-proteasome pathway applies, which has to be unraveled in future. Although SLP65 is a protein with a high turnover, CIN85 increases its protein stability by direct interaction.

\subsubsection{CIN85's stabilization of SLP65 is independent of $\mathrm{Ca}^{2+}$ mobilization}

As shown in figure 3.2.B, CIN85 deficiency leads to reduced $\mathrm{Ca}^{2+}$ signaling upon BCR stimulation. To rule out that this effect is based on reduced SLP65 protein levels, citrinetagged wild-type SLP65 was expressed in CIN85-deficient cells (CIN85 ${ }^{-1}$ SLP65wt). Stimulation with high amounts of anti-lgM $F\left(a b^{\prime}\right)_{2}$ fragments $(10 \mu \mathrm{g} / \mathrm{ml})$ showed that additional expression of SLP65 increased the level of intracellular $\mathrm{Ca}^{2+}$ compared to parental cells (figure 3.13.A). Whereas stimulation with $1 \mu \mathrm{g} / \mathrm{ml}$ anti-lgM $\mathrm{F}\left(\mathrm{ab}{ }^{\prime}\right)_{2}$ fragments revealed that also additional ectopic expression of SLP65 (blue line) was not enough to completely restore $\mathrm{BCR}$-induced $\mathrm{Ca}^{2+}$ mobilization in CIN85-deficient DG75 cells (red line), so that the enhancing influence of CIN85 does not depend on SLP65 amounts in 
that context (figure 3.13.B). This confirms that the observed effect of CIN85 in $\mathrm{Ca}^{2+}$ signaling is not the stabilization of SLP65 protein but rather the support of SLP65 function.

A)

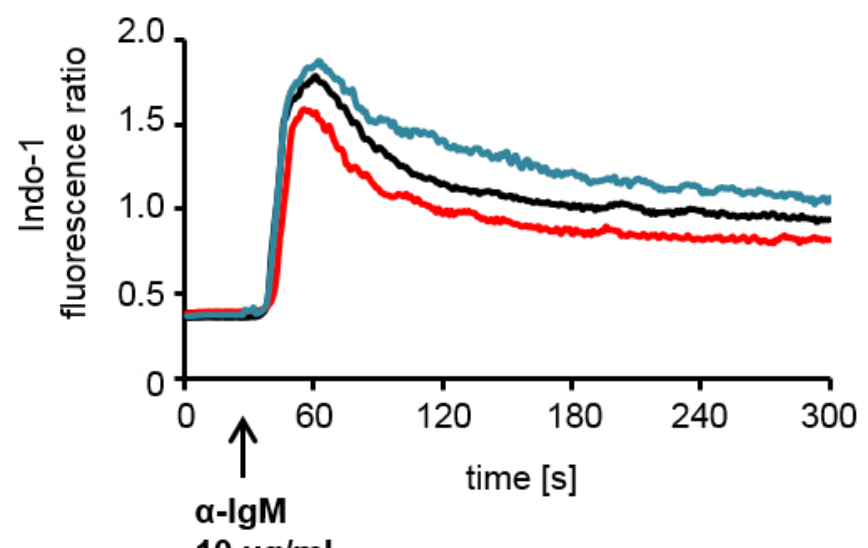

$10 \mu \mathrm{g} / \mathrm{ml}$

DG75

B)

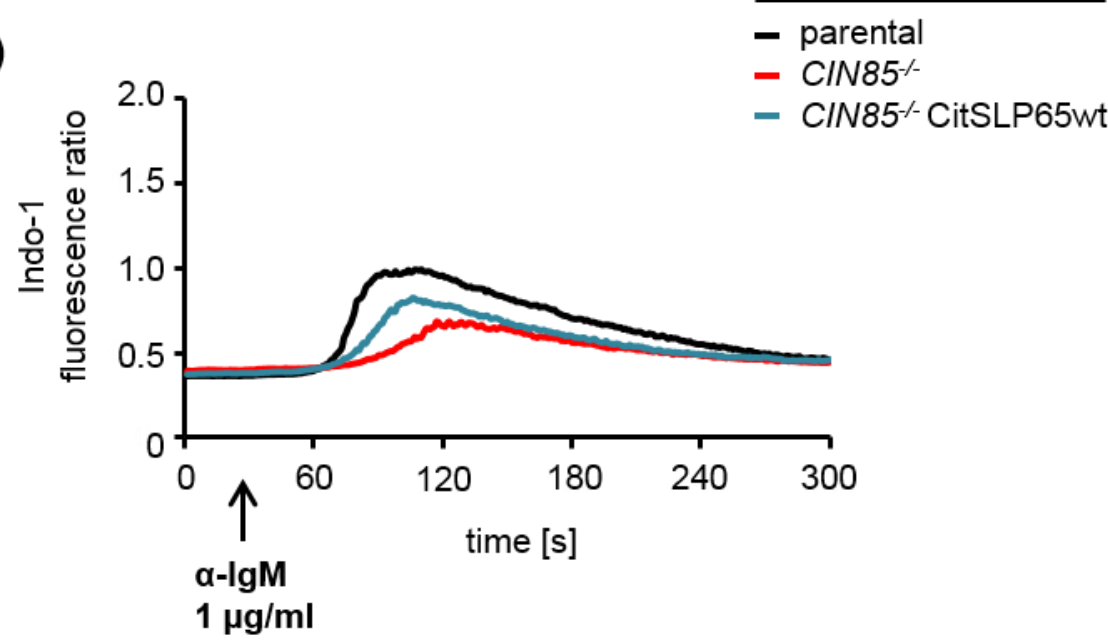

Figure 3.13: SLP65-independent influence on $\mathrm{Ca}^{2+}$ flux of CIN85. DG75 CIN85-deficient cells were retrovirally transduced with constructs coding for citrine-tagged wild-type SLP65. The influence of ectopic SLP65 expression on $\mathrm{Ca}^{2+}$ mobilization in DG75 cells was analyzed upon BCR stimulation with A) $10 \mu \mathrm{g} / \mathrm{ml}$ and B) $1 \mu \mathrm{g} / \mathrm{ml}$ anti-IgM $\mathrm{F}\left(\mathrm{ab}^{\prime}\right)_{2}$ fragments together with parental and $\mathrm{CIN85^{- }}$ cells as before.

\subsection{CIN85 interacts with the short cytoplasmic tail of the IgM-BCR}

\subsubsection{Colocalization of CIN85, SLP65 and the BCR upon BCR stimulation}

It is known that CIN85 and SLP65 are steady ligands (Oellerich et al., 2011). Furthermore, I could show that CIN85 regulates rapid SLP65 membrane recruitment and influences its positive function in $\mathrm{Ca}^{2+}$ mobilization in human $\mathrm{B}$ cells. To visualize this important interaction, constructs encoding RFP-tagged wild-type CIN85 (RFP CIN85) and GFPtagged wild-type SLP65 (GFP SLP65) were virally transduced in DT40 SLP65-deficient 
cells. Analyses of these cells by laser scanning microscopy revealed colocalization of CIN85 and SLP65 (figure 3.14.A).

Previous work of our group showed that CIN85 and also CD2AP are not just recruited to the plasma membrane but also colocalize to BCR containing microclusters after BCR activation (Oellerich et al., 2011). In addition, the adaptor protein SLP65 is also recruited to the BCR after stimulation, where it directly interacts with a phosphorylated non ITAM tyrosine in the Iga chain (Engels et al., 2001). That's why the question arose, whether there is colocalization of these three BCR signaling components.

A)

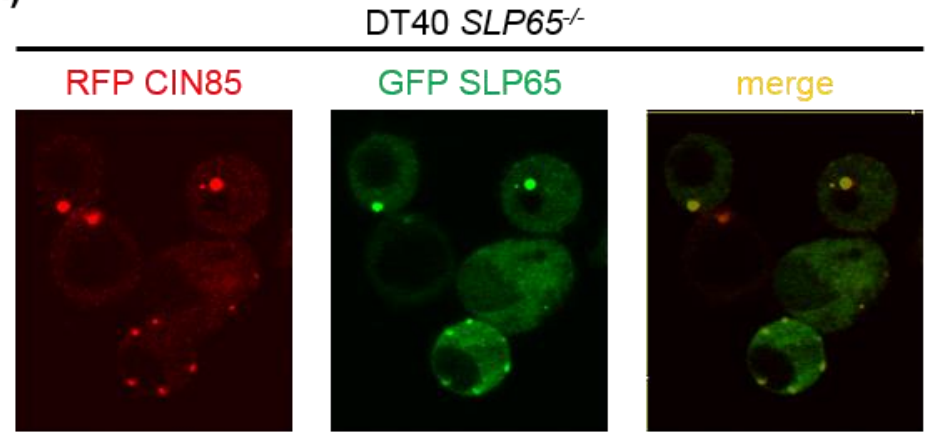

B) DT40 SLP65
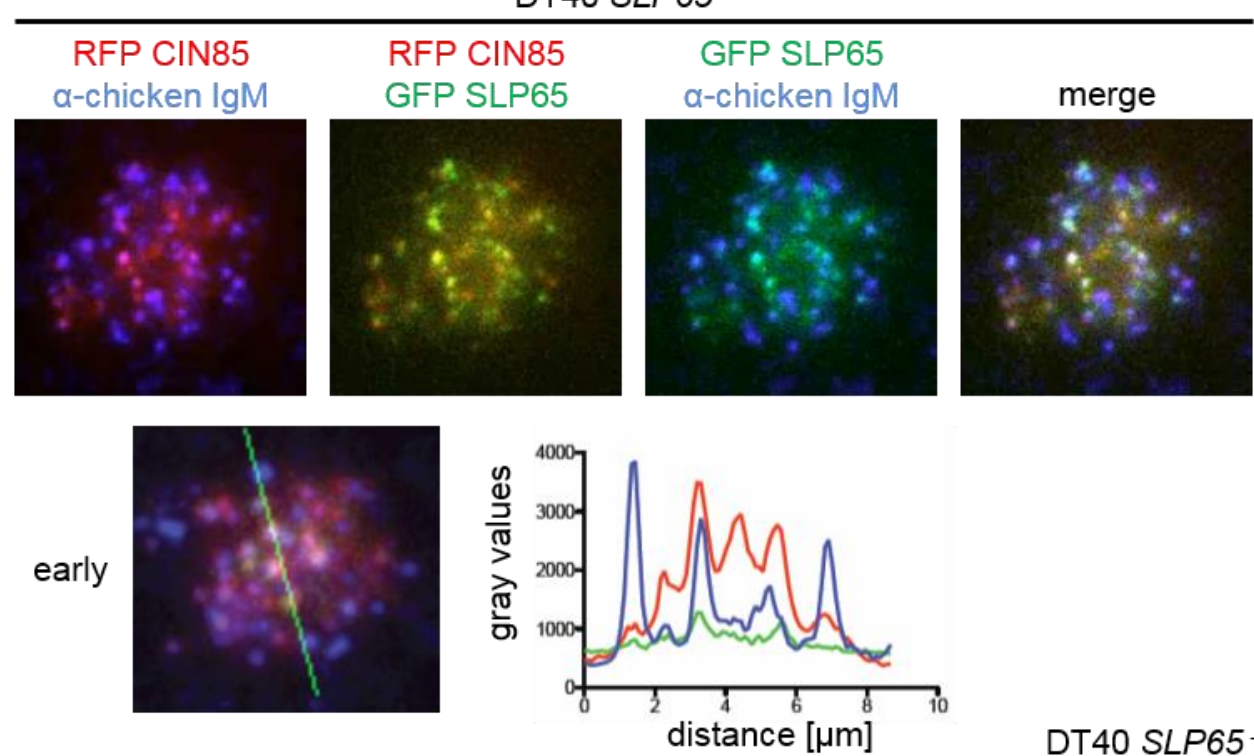

DT40 SLP65\%
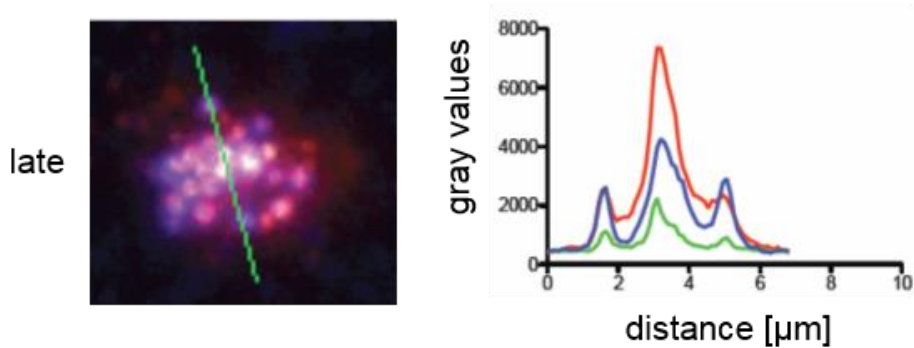

- RFP CIN85

- a-lgM

- GFP SLP65 
Figure 3.14: CIN85, SLP65 and the BCR colocalize after BCR activation. A) DT40 SLP65-deficient cells expressing RFP-tagged wild-type CIN85 (RFP CIN85) and GFP-tagged wild-type SLP65 (GFP SLP65) were subjected to laser scanning microscopy. Data were analyzed with ImageJ. B) The cells described in A) were settled on planar lipid bilayers containing fluorphore-tagged anti-lgM antibodies and analyzed by TIRF microscopy in the group of Facundo Batista.

Therefore, SLP65-deficient DT40 cells expressing citrine-tagged wild-type SLP65 and RFP-tagged wild-type CIN85 were analyzed by total internal reflection (TIRF) microscopy in cooperation with the lab of Facundo Batista (Lymphocyte Interaction Laboratory, Cancer research UK, London). Since DT40 cells are an established model for analyzing localization of fluorophore-tagged proteins upon BCR stimulation by TIRF (Weber et al., 2008) and SLP65-deficient DT40 cells were available, the colocalization experiments were performed in the chicken B cell line. Another advantage of this cell line is that recruitment processes can be monitored also when proteins are additionally expressed. For the experiment, a bilayer was used in which fluorophore coupled anti-chicken BCR antibodies were present that could move freely. On this bilayer, the transduced DT40 cells were settled and the anti-chicken $\operatorname{lgM}$ could bind to and stimulate the membrane resident BCRs. This stimulation mediated recruitment of SLP65 and CIN85 to the activated BCR. All the components did colocalize to a high degree with onset of stimulation (figure 3.14.B). For the equivalent experiment with DT40 cells expressing RFP-tagged CD2AP instead of CIN85, overlapping signals were detected but to a lesser extent than obtained for CIN85 (data not shown). Collectively, these data show that colocalization of CIN85 and SLP65 occurs at the site of BCR-containing microclusters.

\subsubsection{CIN85 interacts with the cytoplasmic part of the IgM-BCR heavy chain}

To confirm the results obtained by TIRF microscopy in another approach, affinity purification of the surface BCR was performed. Therefore, DT40 B cells expressing citrine-tagged wild-type CIN85 (CitCIN85) or CD2AP (CitCD2AP) were used. The immunoblot was stained with an anti-GFP and anti-chicken IgM antibody (figure 3.15.A). CIN85 could be precipitated with the BCR whereas most of CIN85 was likewise purified in $\mathrm{BCR}$ and pervanadate stimulated cells. But for these two samples less IgM was purified which might be because activation of BCRs leads to their aggregation and hence insolubility. In the case of CD2AP this interaction was neither detectable for resting nor pervanadate stimulated cells where also less IgM was purified. But comparison between IgM purifications after BCR activation for 2 min again indicate CIN85 to be associated with the BCR more tightly than CD2AP. 
A)

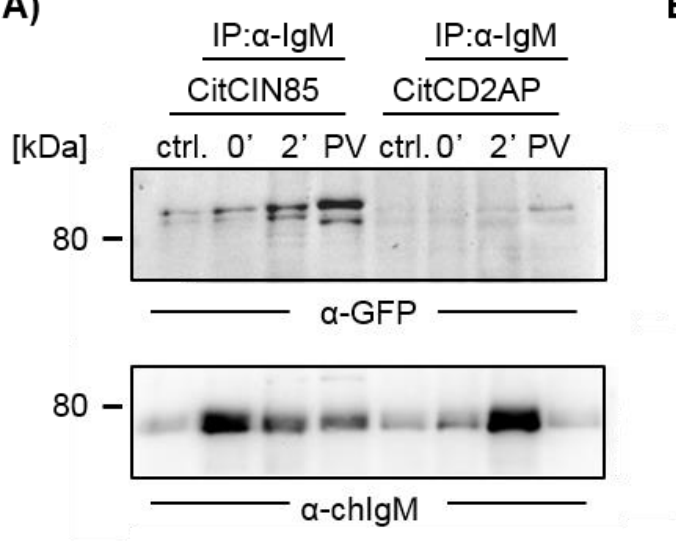

B)

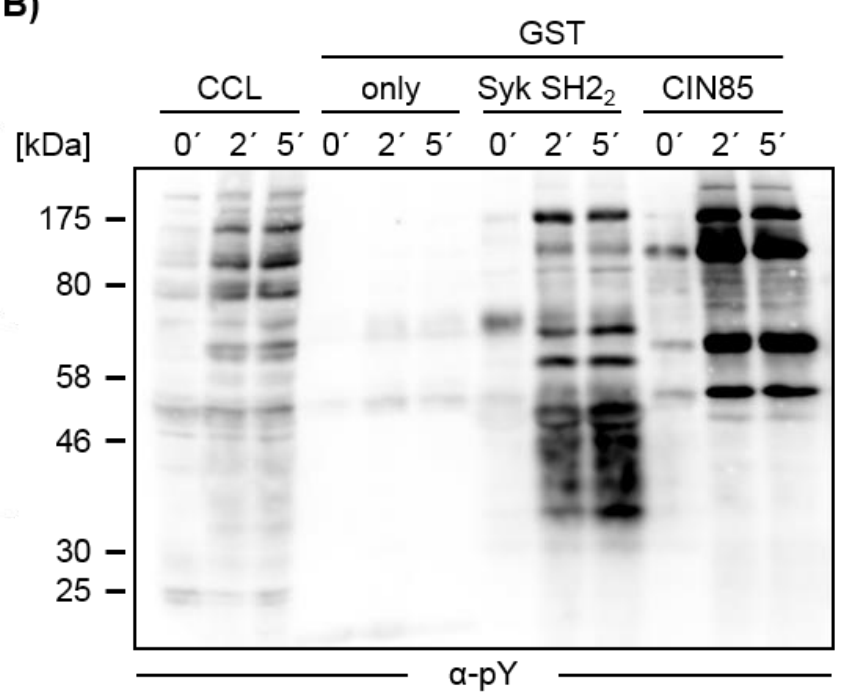

Figure 3.15: CIN85 associates with the IgM-BCR independent of the $\lg \alpha / \lg \beta$ heterodimer. A) DT40 cells expressing citrine-tagged CIN85 or CD2AP were treated as controls (only M4, without goat anti-mouse secondary antibody), stimulated with $2 \mu \mathrm{g} / \mathrm{ml}$ anti-lgM (M4) (including the secondary antibody) or pervanadate (PV). Cleared cellular lysates (CCL) were prepared and either directly subjected to immunoblotting or further used for affinity purification of the BCR. The immunoblot was probed with anti-GFP that recognizes citrinetagged proteins and anti-chicken IgM as control. B) Cleared cellular lysates of Ramos cells were directly used for immunoblotting or further used for affinity purification with GST-only, GST-Syk SH2 2 and GST-CIN85. Samples were subjected to western blot analysis with antibodies against phosphorylated tyrosine ( $p Y)$, Lyn and Syk. The respective molecular weight of marker proteins (in $\mathrm{kDa}$ ) is indicated on the right.

As the cytoplasmic tail consists in the case of the IgM-BCR of only three amino acids, interaction of a ligand like CIN85 is rather likely to appear with the cytoplasmic tails of the $\lg \alpha / \lg \beta$ heterodimer. The $\lg \alpha / \lg \beta$ heterodimer constitutes the main recruitment area of the BCR after activation and might serve as CIN85 interaction site. To test this hypothesis affinity purification experiments with GST-CIN85 were performed in the human B cell line Ramos. As a positive control for Iga/ $\beta$ subunit interaction GST-SykSH2 $2_{2}$ was used, which is known to bind to the heterodimer upon BCR activation (Fütterer et al., 1998). For GSTSykSH $2_{2}$ binding to the phosphorylated Iga/ $\beta$ was shown by probing the immunoblot with anti-phospho tyrosine antibodies (figure 3.15.B). This pattern of the phosphorylated Iga/ $\beta$ subunit was not detectable when GST-CIN85 was used. In summary, for CIN85 association with the $B C R$ the $\lg \alpha / \lg \beta$ heterodimer does not contribute, rather the $\lg M$ heavy chain cytoplasmic tail is involved. 
3.5.3 The cytoplasmic part of the IgM heavy chain is ubiquitinylated upon BCR stimulation

Since CIN85 colocalizes with the BCR upon activation, the question arose whether CIN85 might be recruited via direct $\mathrm{BCR}$ interaction. The $\lg \mathrm{M}$ heavy chain is described to be ubiquitinylated in mice (Drake et al., 2006) and CIN85 is based on nuclear magnetic resonance spectroscopy (NMR) reported to bind to ubiquitin (Bezsonova et al., 2008). Therefore, a direct CIN85-BCR interaction might depend on BCR ubiquitinylation.

The short cytoplasmic tail of the IgM consists of only three amino acids, namely lysine, valine, lysine $(\mathrm{KVK})$, which are consistent throughout evolution and the other immunoglobulin isotypes, except IgA. To be able to analyze BCR ubiquitination and its effects in BCR signaling, a system developed by Niklas Engels was used. Here, the extracellular part of a murine $\mathrm{y} 2 \mathrm{a}(\mathrm{m} / 2 \mathrm{a})$ is coupled to the intracellular part of the human $\lg \mathrm{M}(\mathrm{KVK})$. In this system, one can modify the intracellular part of the $\lg \mathrm{M}$ and visualize the signaling effects by stimulation with an anti-mlgG antibody (figure 3.16.A).

A)
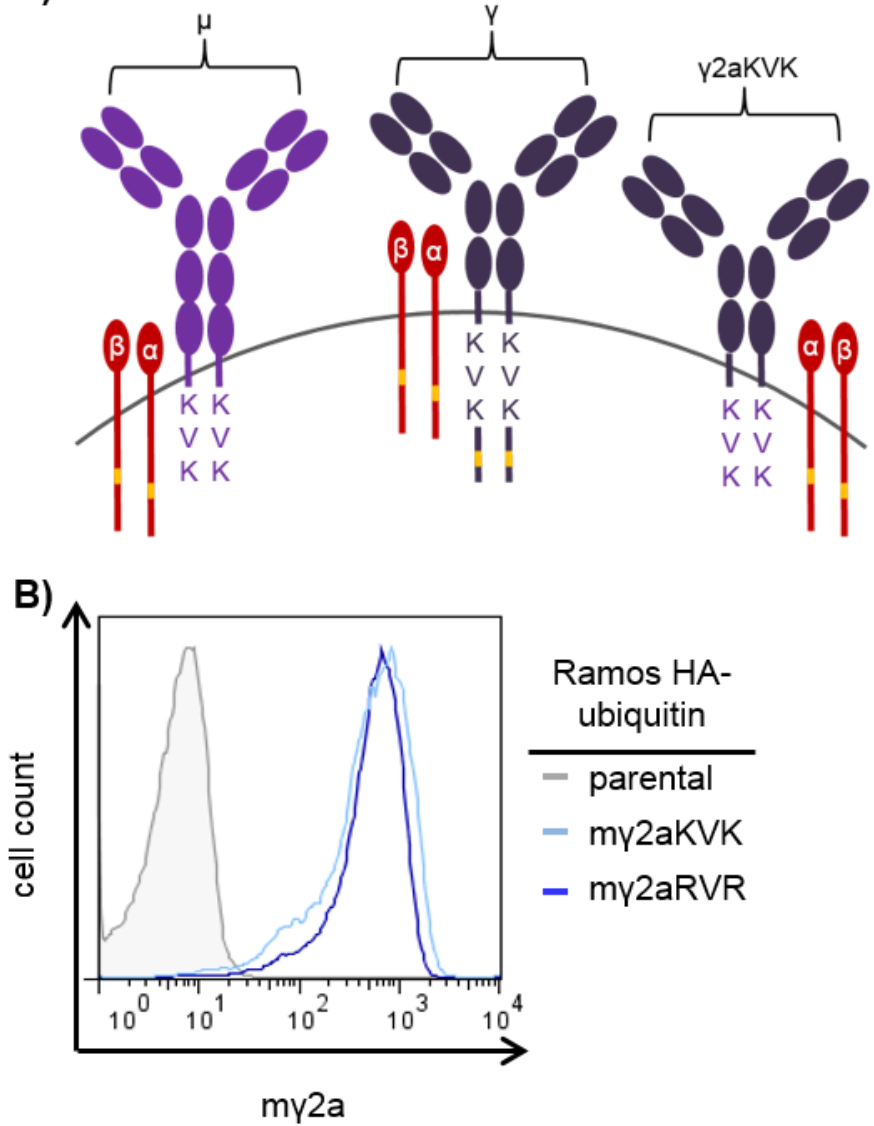
C)

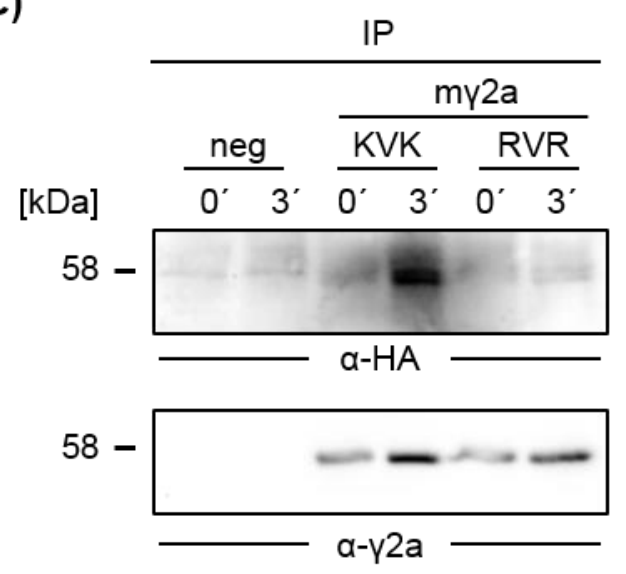

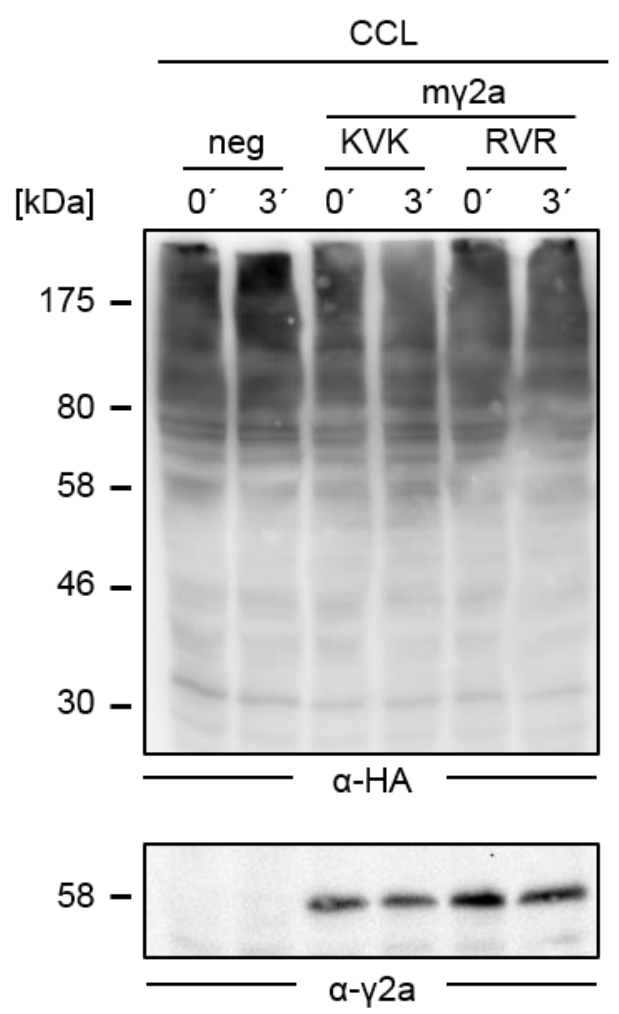

Figure 3.16: The amino acid sequence KVK in the cytoplasmic tail of the BCR is ubiquitinylated. A) Schematic drawing of the $\operatorname{lgM}(\mu)$, $\lg G(\mathrm{y})$ and chimeric my2aKVK BCR's. B) Flow cytometric analysis of surface expression levels of my2aKVK and my2aRVR BCR's in Ramos cells. C) Cleared cellular lysates of resting and mlgG stimulated Ramos cells expressing HA-ubiquitin, HA-ubiquitin and Y2aKVK or HA-ubiquitin and Y2aRVR were prepared and either directly subjected to immunoblotting (right panels) or subjected to affinity purification of surface my2a. The blots were probed with antibodies detecting HA-tag or my2a. The respective molecular weight of marker proteins (in $\mathrm{kDa}$ ) is indicated on the left.

Lysine is a basic amino acid that can be modified by ubiquitinylation. Arginine $(R)$ is also a basic and resembles lysine the most. However, arginine is not modified by ubiquitinylation. To show that the surface BCR is ubiquitinylated at its cytoplasmic tail in human B cells, I used the my2aKVK construct and substituted the lysines with arginines resulting in the $\mathrm{y} 2 \mathrm{aRVR}$ construct. $\mathrm{B}$ cells expressing eight linked ubiquitins fused with an HA-tag were used for viral transduction of my2aKVK or my2aRVR constructs. The cells were analyzed for equal surface expression of BCR variants with flow cytometry (figure 3.16.B). As control, cells negative for my2a but positive for HA-ubiquitin were used. Affinity purification of my2aKVK showed ubiquitinylation of the y2aKVK heavy chain after stimulation as the ubiquitin signal is exactly at the same molecular weight as the signal of the my2a heavy chain (figure 3.16.C). In contrast, the my2aRVR heavy chain was neither ubiquitinylated in resting nor in stimulated cells. This proved that the reported BCR ubiquitinylation at the cytoplasmic tail of the IgM heavy chain in mice (Drake et al., 2006) 
also occurs in our human cell system and can be circumvented by lysine to arginine substitution.

\subsubsection{CIN85 interacts via its SH3 domains with ubiquitin}

CIN85 was first described as a cbl interacting protein. The interaction partner cbl is an E3 ubiquitin protein ligase that transfers ubiquitin from an E2 ubiquitin-conjugating protein to a substrate, which is thereby marked for proteasomal degradation. This fact and the findings that the CIN85 SH3 domains are capable of binding to ubiquitin in vitro (Stamenova et al., 2007) let us hypothesize that CIN85 is involved in the ubiquitin pathway. CIN85 binding to ubiquitin was detected by NMR and revealed weak interaction (Bezsonova et al., 2008). To test this interaction in a biochemical approach, I used GSTCIN85 fusion proteins with either one of the $\mathrm{SH} 3$ domains $(\mathrm{A}, \mathrm{B}, \mathrm{C})$ or the $\mathrm{C}$-terminal part of the protein harboring the proline-rich region and the CC domain (PRC) and performed affinity purification experiments in the human B cell line Ramos (figure 3.17.A). For all three SH3 domains the typical complex pattern of polyubiquitinylated proteins of different size that appear like a "smear" on the immunoblot was obtained by precipitation after BCR stimulation. In addition, a prominent signal at the size of approximately $80 \mathrm{kDa}$ was present that might be the ubiquitinylated cbl or lgM heavy chain. For the C-terminal part of CIN85 that characteristic ubiquitin "smear" was not present but rather a strong signal at the size of the GST-CIN85PRC fusion protein itself. A possibility could be that the fusion protein itself is an ubiquitin acceptor, meaning that a lysine in the C-terminus of CIN85 can be ubiquitinylated (Haglund et al., 2002). Another explanation could be that the Cterminus interacts with an ubiquitinylated protein probably independent of ubiquitin as a modification. With this experiment, I could show that the SH3 domains of CIN85 can interact with ubiquitinylated proteins. Since this is no evidence for direct binding to ubiquitin, I purified ubiquitin binding proteins from cleared cellular lysate from resting and BCR stimulated Ramos cells using ubiquitin-coupled agarose and tested for CIN85 association (figure 3.17.B). Indeed, a weak interaction of CIN85 with ubiquitin could be shown whereas SLP65 could not be detected as an ubiquitin interaction partner. The protein c-cbl was used as positive control in this experiment, because it is described to bind to ubiquitin (Robertson et al., 2000; Davies et al., 2004). Also Syk and the heavy chain of the mlgM were found to interact with ubiquitin whereas the BCR signaling component Iga did not. These biochemical results obtained in human B cells confirmed the interaction between the SH3 domains of CIN85 and the lysine modification ubiquitin. 
A)
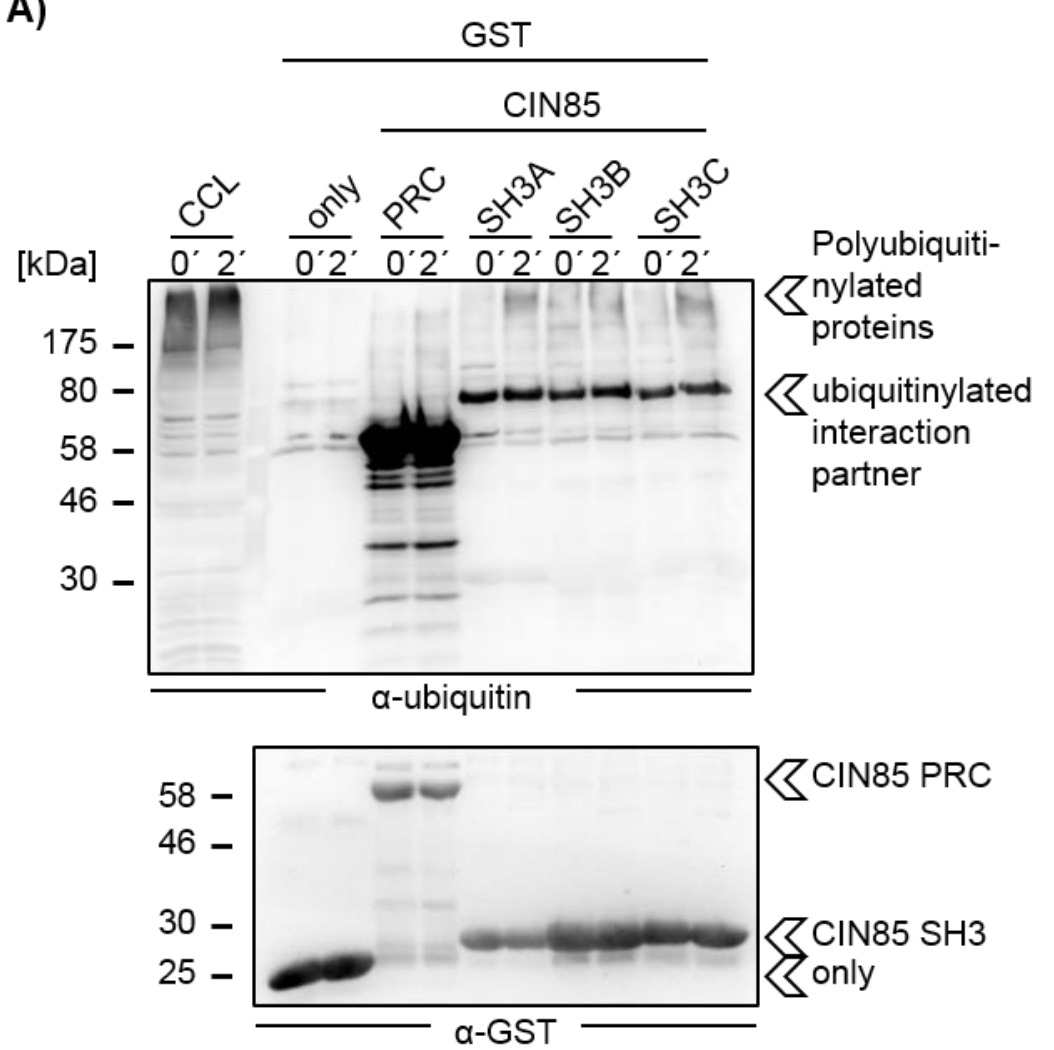

B)

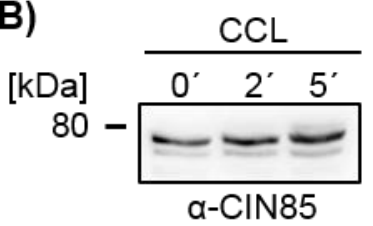

\begin{tabular}{cc} 
ubiquitin-AP \\
\hline $0^{\prime} \quad 2^{\prime} 5^{\prime}$
\end{tabular}
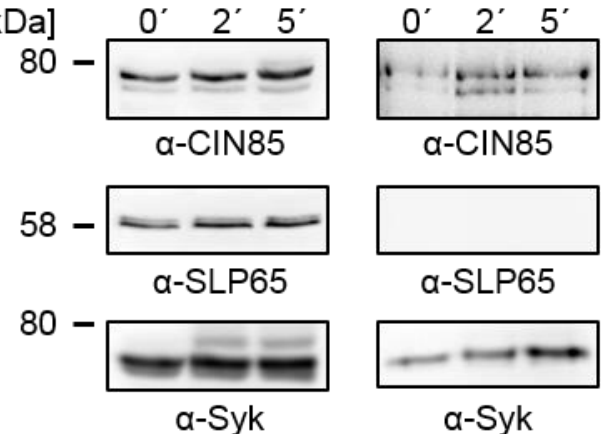

a-Syk

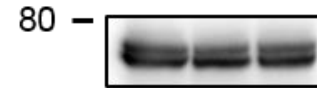

$\alpha-\lg M$

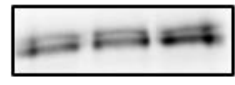

a-lgM

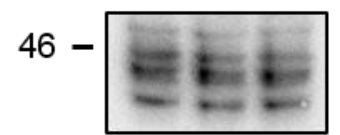

a-Igalpha
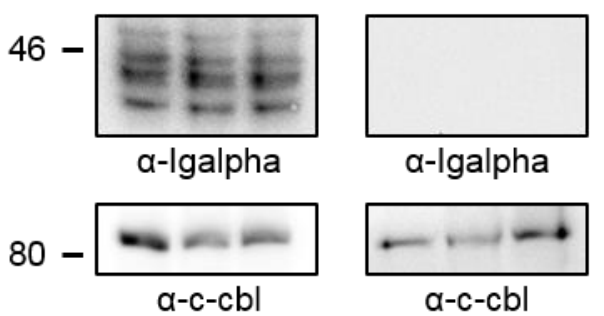

a-Igalpha

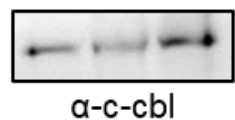

Figure 3.17: CIN85 can interact with ubiquitin. A) Cleared cellular lysates of resting and BCR stimulated $\left(10 \mu \mathrm{g} / \mathrm{ml}\right.$ anti-lgM $\mathrm{F}\left(\mathrm{ab}^{\prime}\right)_{2}$ fragments) Ramos cells were prepared and either directly subjected to immunoblotting or further used in affinity purification experiments with GST-only, the three separated SH3 domains (GST-SH3A, GST-SH3B, GST-SH3C) or the C-terminal part of CIN85, namely the proline-rich part 
with the CC domain (GST-PRC). The blot was stained with anti-Ubiquitin and anti-GST antibodies. B) Cleared cellular lysates of resting and for $2 \mathrm{~min}\left(2^{\prime}\right)$ and $5 \mathrm{~min}\left(5^{\prime}\right)$ stimulated $\left(10 \mu \mathrm{g} / \mathrm{ml}\right.$ anti-lgM $\mathrm{F}\left(\mathrm{ab}^{\prime}\right)_{2}$ fragments) Ramos cells were either directly subjected to immunoblotting (left panels) or further used for affinity purification experiments using ubiquitin coupled to agarose. The blot was probed with antibodies detecting CIN85, SLP65, Syk, IgM, Igalpha or c-cbl. The respective molecular weight of marker proteins (in kDa) is indicated on the left.

\subsubsection{IgM heavy chain ubiquitinylation enhances CIN85 interaction with the BCR}

Since I could show that the cytoplasmic tail of the IgM heavy chain is ubiquitinylated and that CIN85 is capable of binding to ubiquitin in human B cells, I wanted to determine whether the CIN85-BCR interaction is mediated by this modification. Therefore, I performed surface BCR affinity purification experiments using four different chimeric BCRs (my2aKVK, RVK, KVR or RVR), which were expressed in Ramos cells via retroviral transduction. Cell sorting was applied to achieve comparable surface expression (figure 3.18.A). Ramos cells lacking my2a expression served as a negative control. BCR purification of stimulated cells revealed strongest interaction of CIN85 with the my2aKVK BCR (figure 3.18.B). Weaker signals were visible for my2aRVK and my2aKVR but for these samples also less BCR was precipitated. The lowest amount of CIN85 was copurified by precipitation of my2aRVR.

An additional check with anti-phospho tyrosine antibodies to detect possible differences in phosphorylation and hence activation of BCR interacting proteins revealed decreased interaction with the tyrosine phosphorylated $\lg \alpha / \lg \beta$ heterodimer in the my2aRVK and my2aRVR expressing cells. Comparison with overall phosphorylation patterns of the cleared cellular lysates showed that $\lg \alpha / \lg \beta$ phosphorylation did occur. This indicates that the interaction between the BCR heavy chain and the $\lg \alpha / \lg \beta$ heterodimer was weakened due to cytoplasmic tail amino acid substitutions. To elucidate this observed effect of the RVR cytoplasmic tail further, a BCR purification was performed in resting as well as stimulated cells expressing either $\mathrm{y} 2 a K V K$ or $\mathrm{Y} 2 \mathrm{aRVR}$. Loss of Iga interaction in resting and BCR stimulated y2aRVR expressing cells (figure 3.18.C) confirmed the loss of complex stability upon BCR purification experiments and further indicated that it is independent of BCR-stimulation. Collectively, the membrane proximal lysine in KVK is responsible for complex stability as also $\mathrm{Y} 2 \mathrm{aRVK}$ precipitated less $\lg \alpha / \lg \beta$. However, this BCR complex instability for Y2aRVK did not influence CIN85-BCR interaction. In conclusion, the lysine residues in the cytoplasmic part of the BCR that are ubiquitinylated upon BCR activation, determine interaction with CIN85 and additionally stabilize the BCR multi-protein complex. 
A)

$$
\begin{aligned}
& \text { Ramos } \\
& \hline \text { - parental } \\
& \text { - my2aKVK } \\
& \text { - my2aRVK } \\
& \text { - my2aKVR } \\
& \text { - my2aRVR }
\end{aligned}
$$

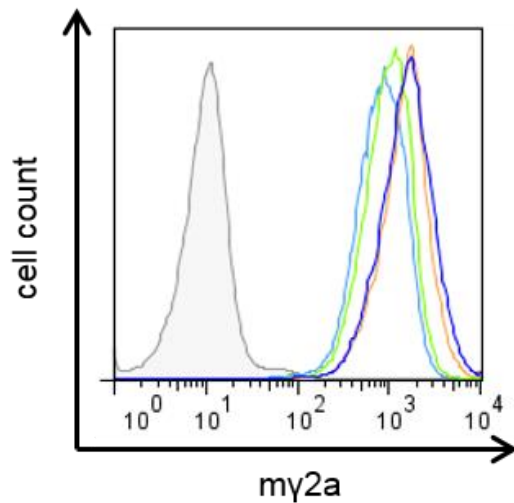

C)

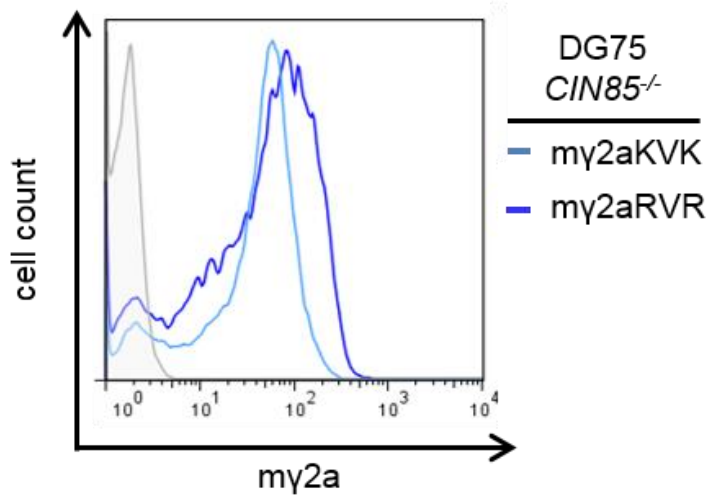

B) $\mathrm{CCL}$

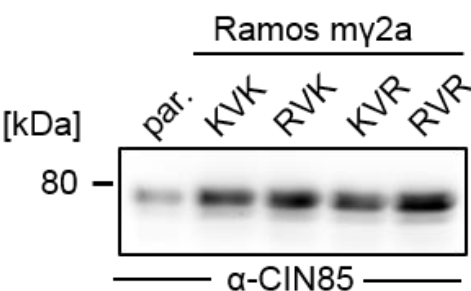

58
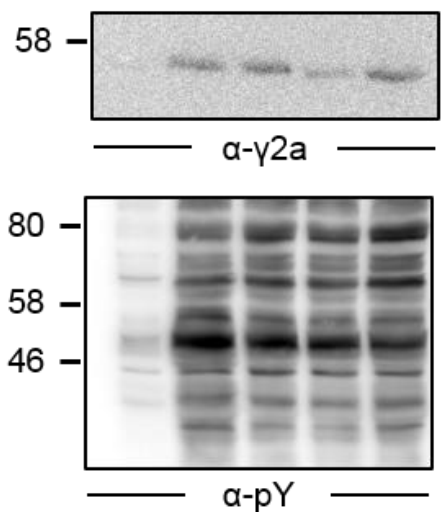

IP

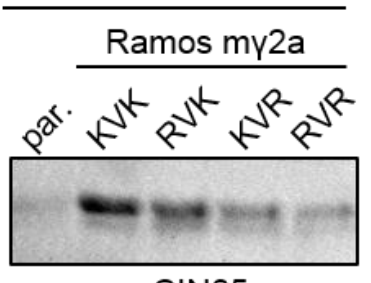

a-CIN85

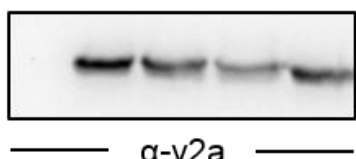

$\mathrm{a}-\mathrm{\gamma} 2 \mathrm{a}$

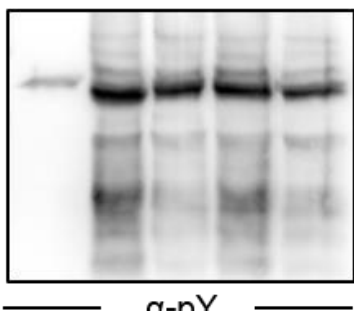

$\alpha-p Y$

D)

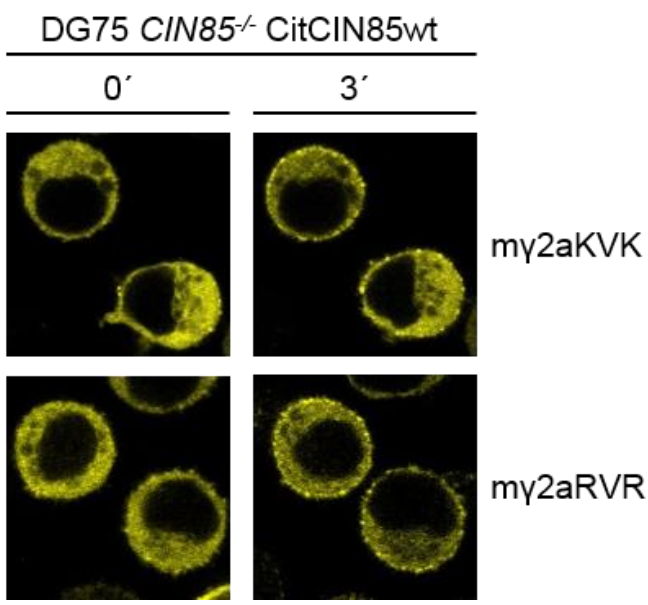

Figure 3.18: Ubiquitinylation of the BCR influences its interaction with CIN85 but is negligible for CIN85 plasma membrane recruitment. A) Ramos cells were retrovirally transduced to express constructs encoding my2aKVK, my2aRVK, my2aKVR or my2aRVR. Surface expression levels of the my2a constructs were analyzed by flow cytometry after cell sorting. B) Cleared cellular lysates of the cells described in A) were prepared after stimulation for 2 min with $10 \mu \mathrm{g} / \mathrm{ml}$ anti-mlgG $\mathrm{F}\left(\mathrm{ab}^{\prime}\right)_{2}$ fragments. Ramos cells, which did not express the my2a, served as negative control. Lysates were either directly subjected to immunoblotting or used for affinity purification experiments of the my2a. The respective molecular weight of marker proteins (in $\mathrm{kDa}$ ) is indicated on the left. C) CIN85-deficient DG75 cells were retrovirally transduced with constructs encoding my2aKVK or my2aRVR. Surface expression levels of my2a BCR variants were analyzed with flow cytometry. D) Cells described in C) were retrovirally transduced with constructs coding for citrine-tagged wildtype CIN85 (CitCIN85wt) and subjected to confocal laser scanning microscopy. CIN85 plasma membrane recruitment was analyzed upon stimulation with $10 \mu \mathrm{g} / \mathrm{ml}$ anti-mlgG $\mathrm{F}\left(\mathrm{ab}^{\prime}\right)_{2}$ fragments. 
The input of the cytoplasmic tail in CIN85-BCR interaction raised the question whether it also impacts CIN85 plasma membrane recruitment. To investigate this question, the cell line was changed to CIN85-deficient DG75 cells because recruitment of additionally expressed proteins is barely visible in the here used Ramos human burkitt lymphoma cell line. CIN85-deficient DG75 cells expressing citrine-tagged wild-type CIN85 were retrovirally transduced to express my2aKVK or my2aRVR on their cell surface and expression levels of BCR variants were tested by flow cytometry (figure 3.18.D). These cells were analyzed with the laser scanning microscopy after stimulation with $10 \mu \mathrm{g} / \mathrm{ml}$ $F\left(a b^{\prime}\right)_{2}$ fragments anti-mlgG. Citrine-tagged CIN85 was first located in the cytoplasm and recruited to the plasma membrane upon stimulation of my2aKVK (figure 3.18.E). Translocation of CIN85 was also present in my2aRVR expressing cells so that plasma membrane recruitment of CIN85 is independent of the conserved amino acid sequence KVK. This indicates that the cytoplasmic part of the IgM heavy chain does not mediate plasma membrane recruitment but BCR interaction with CIN85.

\subsubsection{BCR-induced tyrosine phosphorylation and $\mathrm{Ca}^{2+}$ response are independent} of KVK

To test whether the cytoplasmic tail has an influence on signaling onset, Ramos cells expressing the four different chimeric receptors (compare figure 3.18.A) were stimulated with anti-mlgG $\mathrm{F}\left(\mathrm{ab} \mathrm{b}_{2}\right)_{2}$ fragments and cleared cellular lysates were prepared. Staining of the immunoblot with anti-phospho tyrosine antibodies showed the same kinetics of phosphorylation events in these cells (figure 3.19.A). A detailed look at the phosphorylation status of the kinases Syk and Erk and also the adaptor protein SLP65 did not reveal any changes between the chimeric BCRs (figure 3.19.B).

Furthermore, $\mathrm{Ca}^{2+}$ mobilization as further downstream readout for signaling capability was analyzed. $\mathrm{Ca}^{2+}$ flux was initiated either by stimulation of the different chimeric my2a or the endogenous IgM BCR. $\mathrm{Ca}^{2+}$ mobilization upon stimulation of the endogenous BCR was similar in all analyzed cells (figure 3.19.C). In contrast, $\mathrm{Ca}^{2+}$ mobilization in cells expressing my2aRVK and my2aRVR was moderately enhanced compared to my2aKVK expressing cells (figure 3.19.D). This enrichment resembled the elevated my2a surface expression of my2aRVK and my2aRVR (compare figure 3.18.A) and is probably conditioned on that. Collectively, the three amino acids KVK in the cytoplasmic tail did not show signaling capability in BCR-induced phosphorylation events and $\mathrm{Ca}^{2+}$ response. 

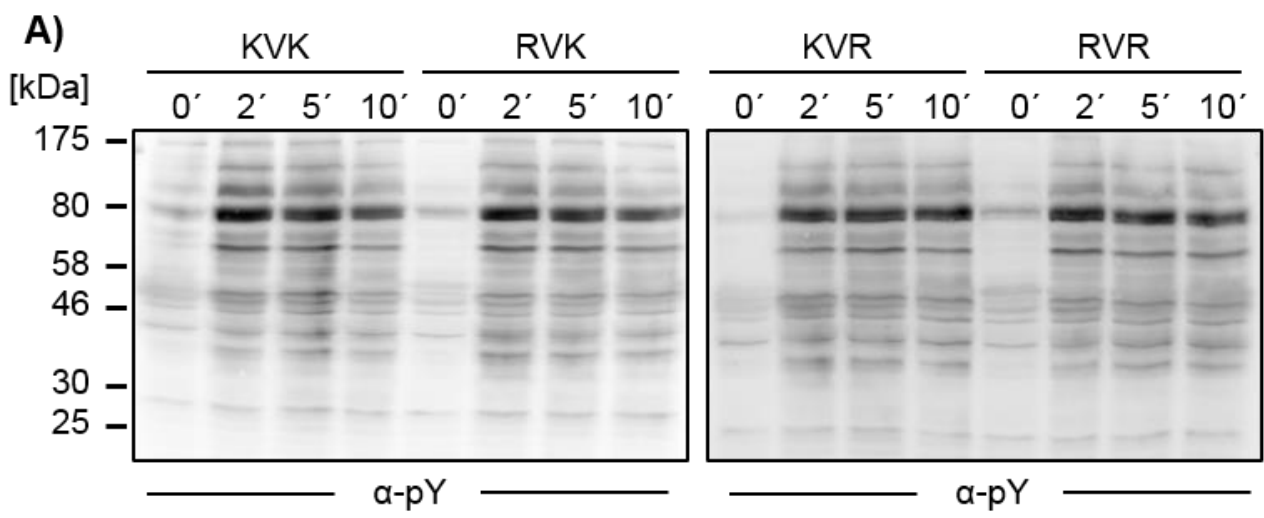

B)

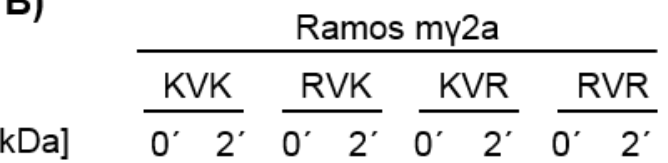

C)
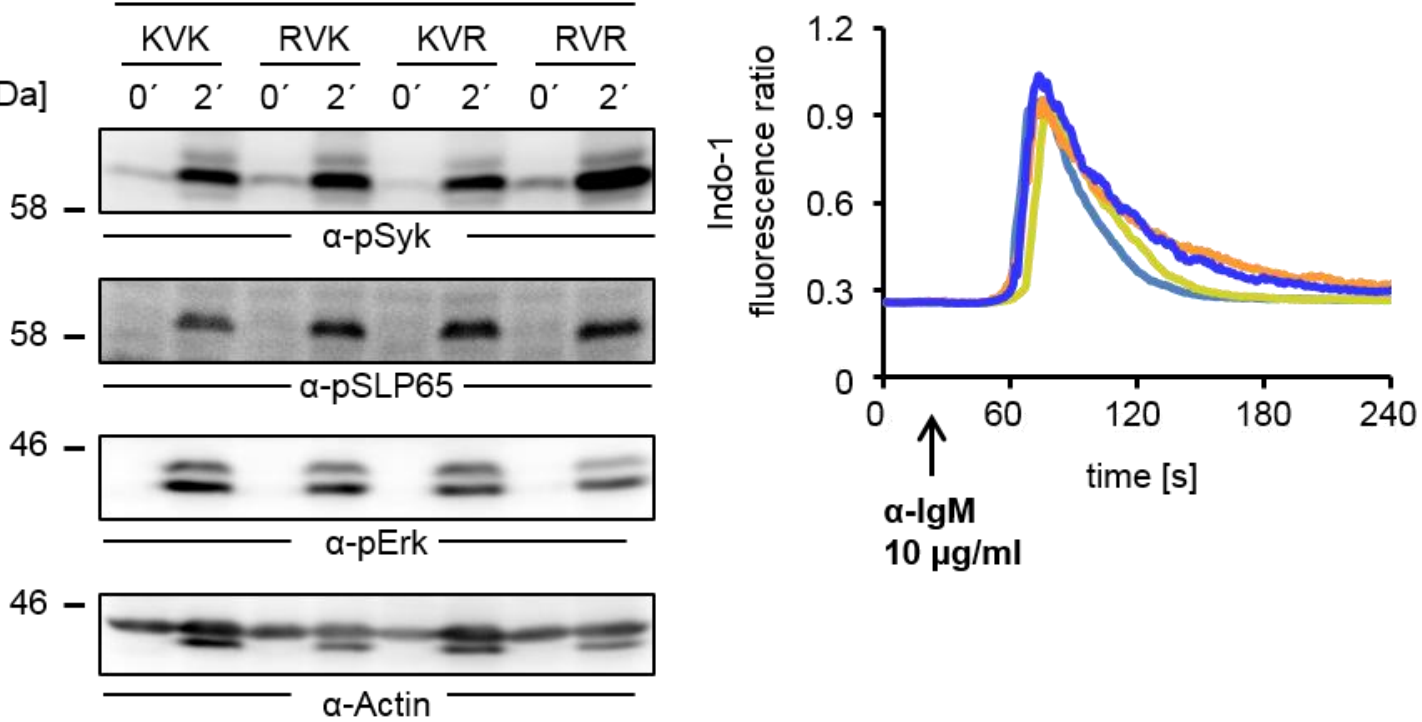

D)
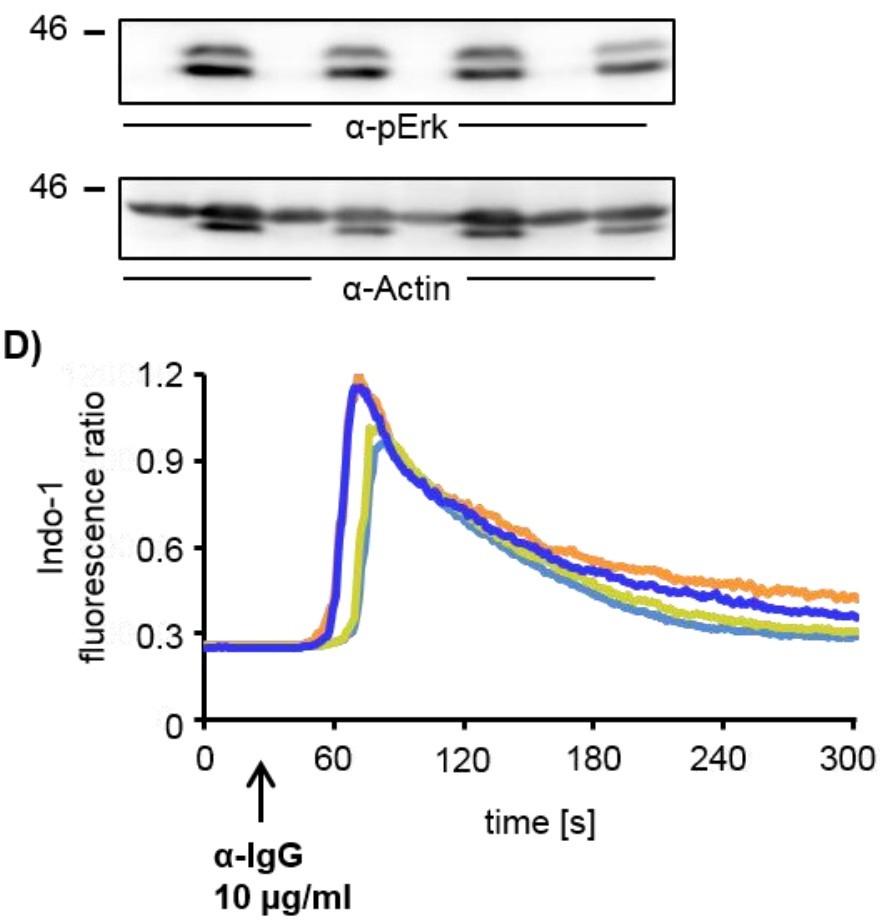

$$
\begin{aligned}
& \text { Ramos } \\
& \hline- \text { my2aKVK } \\
& - \text { my2aRVK } \\
& - \text { my2aKVR } \\
& - \text { my2aRVR }
\end{aligned}
$$

Figure 3.19: The cytoplasmic amino acids KVK of the BCR heavy chain did not reveal signaling capability in terms of tyrosine phosphorylation and $\mathrm{Ca}^{2+}$ mobilization. A) Cleared cellular lysates of resting $\left(0^{\prime}\right)$ and stimulated chimeric my2a expressing Ramos cells for $2 \mathrm{~min}\left(2^{\prime}\right), 5 \mathrm{~min}\left(5^{\prime}\right), 10 \mathrm{~min}\left(10^{\prime}\right)$ with $10 \mu \mathrm{g} / \mathrm{ml}$ anti-mlgG $\mathrm{F}\left(\mathrm{ab}^{\prime}\right)_{2}$ fragments were prepared, subjected to SDS-PAGE and the immunoblot was probed with anti-phospho tyrosine antibodies. B) Cleared cellular lysates of resting $\left(0^{\prime}\right)$ and stimulated chimeric my2a expressing Ramos cells for 2 min (2') with anti-mlgG $F\left(a b^{\prime}\right)_{2}$ fragments were prepared, subjected to SDS-PAGE and the immunoblot was stained for phosphorylated Syk, SLP65, Erk and Actin as 
control. The respective molecular weight of marker proteins (in $\mathrm{kDa}$ ) is indicated on the left. C, D) $\mathrm{Ca}^{2+}$ mobilization of Ramos cells expressing my2a was measured as before upon $\lg M(C)$ or $\mathrm{mlg} G(D)$ stimulation with $10 \mu \mathrm{g} / \mathrm{ml}$ of each antibody, respectively.

3.5.7 BCR internalization is not influenced by the KVK cytosolic part of the BCR heavy chain and CIN85

Even though $\mathrm{BCR}$-induced phosphorylation events and $\mathrm{Ca}^{2+}$ mobilization are not influenced by lysine to arginine substitution BCR ubiquitinylation might influence BCR internalization upon BCR stimulation. To analyze this, the previously described chimeric $\mathrm{BCR}$ variants (see chapter 3.5.5) were stimulated for different periods and the amounts of remaining surface BCRs were analyzed by flow cytometry. The same was done with the endogenous IgM BCR as a control.

A)
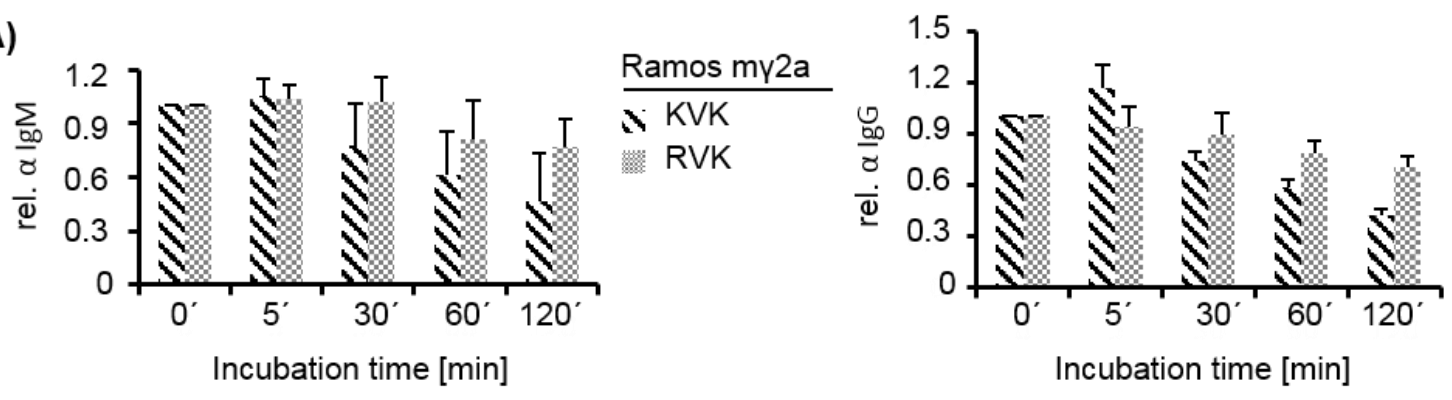

B)
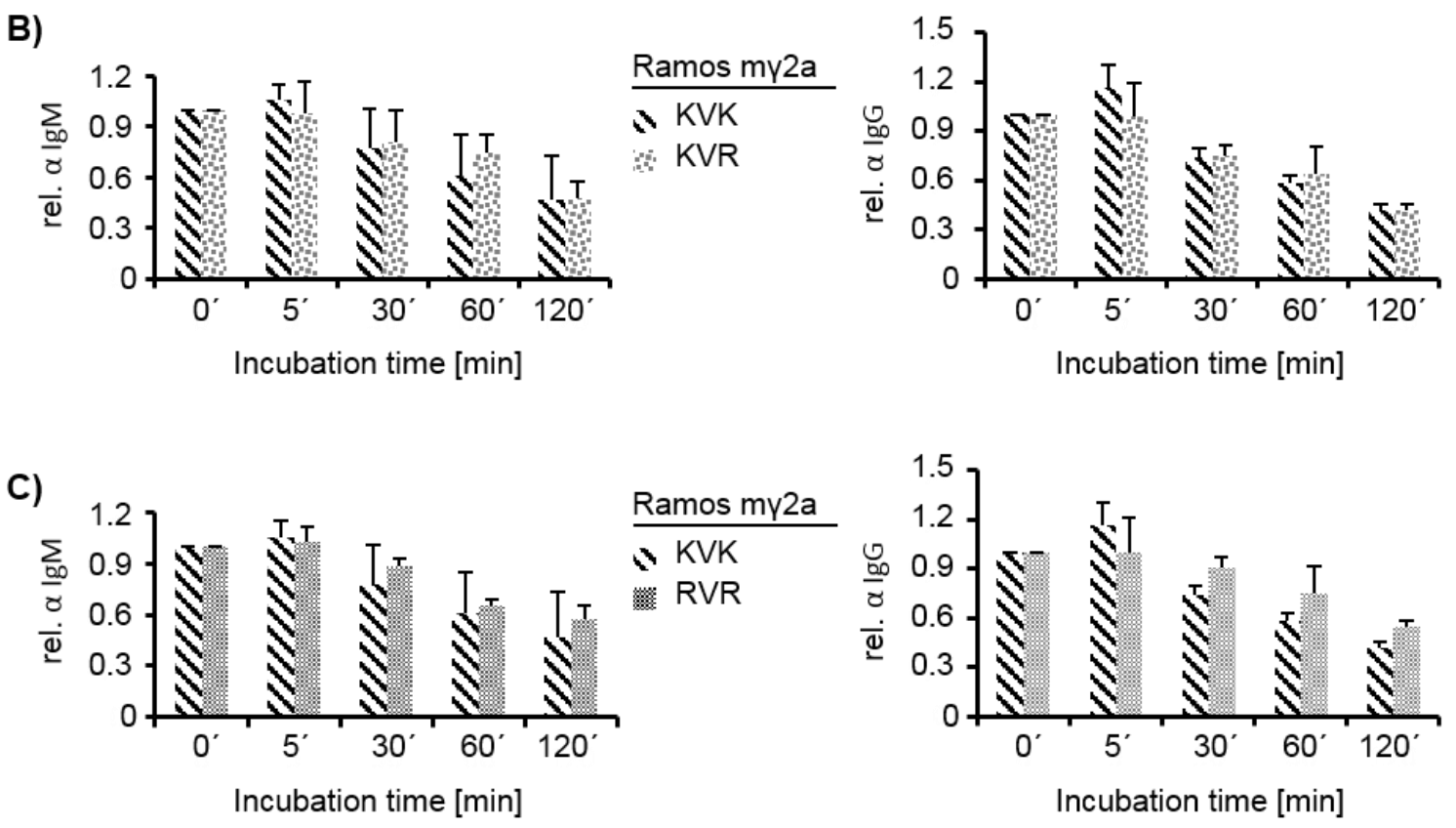

Figure 3.20: The KVK sequence in the BCR heavy chain has no impact on BCR activation-mediated internalization. Ramos my2aRVK (A), my2aKVR (B), my2aRVR (C) expressing cells were analyzed for endogenous surface IgM (left panels) and ectopic surface $\mathrm{mlgG}$ (right panels) before and $5 \mathrm{~min}\left(5^{\prime}\right), 30 \mathrm{~min}$ 
$\left(30^{\prime}\right), 60 \mathrm{~min}\left(60^{\prime}\right)$ and $120 \mathrm{~min}\left(120^{\prime}\right)$ after stimulation of the BCR with $10 \mu \mathrm{g} / \mathrm{ml}$ of biotinylated antibodies. The mean fluorescence intensity of fluorophore-coupled secondary streptavidin antibodies (CY5) was determined using flow cytometry. The amount of surface BCR is shown as relative values compared to the amount of my2aKVK BCRs. Standard deviations were determined from three independent experiments of the calculated relative values.

For my2aRVK (figure 3.20.A) and my2aRVR (figure 3.20.C) small differences between ectopically expressed y2aKVK BCRs (left panels) and the ectopically expressed my2a substitution variants BCRs (right panels) in their internalization rates were visible. However, the differences for these two chimeric my2a BCR variants resembled the pattern for the endogenous IgM internalization profile. The my2aKVR expressing cells internalized IgG and also endogenous IgM similar to the my2aKVK cells (figure 3.20.B). This indicates that the different cell populations reacted divergent to a small degree and that this was the reason for marginal changes in the internalization rates. In conclusion, the above described ubiquitinylation of the KVK (chapter 3.5.3) does not influence BCRinduced internalization.

As CIN85 associates with the BCR upon BCR stimulation (chapter 3.5.1) and involvement of CIN85 in epidermal growth factor receptor (EGFR) internalization is controversially discussed (Havrylov et al., 2010), I tested whether loss of CIN85 impairs BCR internalization. Therefore, CIN85-deficient DG75 cells were stimulated with biotinylated anti-human $\lg \mathrm{M}$ antibodies that were internalized due to BCR stimulation. Residual surface bound BCRs were then stained with fluorophore-conjugated streptavidin antibodies. In this setup, only BCRs were stained that remained on the cell surface after different periods of stimulation. BCR-mediated internalization of both parental and CIN85deficient DG75 cells constituted up to $60 \%$ for the longest time point measured (120 min) (figure 3.21). In addition, all other analyzed time points showed comparable internalization rates. This illustrates that loss of CIN85 in DG75 cells does not influence BCR internalization.

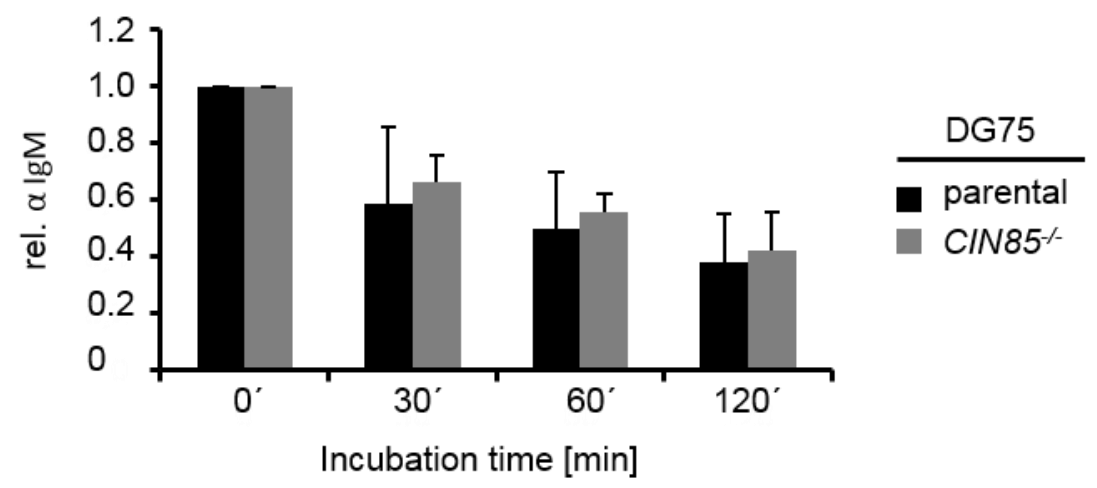


Figure 3.21: Loss of CIN85 does not alter the process of BCR internalization. DG75 parental (black) or CIN85-deficient cells (grey) were analyzed for the amount of surface lgM before and $30 \mathrm{~min}$ (30'), 60 min (60') and $120 \mathrm{~min}\left(120^{\prime}\right)$ after stimulation of the BCR with $10 \mu \mathrm{g} / \mathrm{ml}$ of biotinylated anti-lgM antibodies. The mean fluorescence intensity of fluorophore-coupled secondary streptavidin antibodies (CY5) was determined using flow cytometry. The amount of surface BCR is shown as relative values compared to the BCR amounts of resting $B$ cells. Standard deviations were determined from three independent experiments of the calculated relative values.

To test a possible impact of CIN85s homolog CD2AP in that process, shRNA-mediated knock down of CD2AP in parental and CIN85-deficient DG75 cells was performed to examine turnover of surface $\operatorname{lgM}$ upon BCR stimulation. Neither diminished CD2AP expression alone nor the combined CD2AP reduction with the lack of CIN85 did alter BCR internalization under the here applied conditions (data not shown). This indicates that CIN85 as well as CD2AP are not involved in the process of BCR internalization upon BCR stimulation.

\subsection{CIN85 impact on the NF-KB pathway}

\subsubsection{Enhancing function of CIN85 in the NF-KB pathway}

As shown in chapters 3.2.6 and 3.4.1 CIN85 influences the key adaptor protein SLP65 in terms of $\mathrm{Ca}^{2+}$ flux, plasma membrane recruitment and expression. In addition, it can associate with the BCR and interact with ubiquitin (chapter 3.5.1 and 3.5.4, respectively). Further influence of CIN85 was found in the activation process of the transcription factor nuclear factor kappa-light-chain-enhancer of activated B cells (NF-kB). Here, BCR stimulation of primary $B$ cells of a CIN85-deficient patient could not induce NF-KB activation whereas other stimuli (PMA, CD40) did (unpublished data). Moreover, it is published that mice lacking the expression of CIN85 specifically in B cells have a decreased NF-KB response after BCR stimulation (Kometani et al., 2011).

To elucidate CIN85 influence on the NF-KB pathway in more detail, I used the DT40 B cell line, as activation of NF-KB upon stimulation of the BCR is detectable by immunoblotting in this cell line. Therefore, a shRNA-mediated CIN85 knock down was performed to decrease the amount of CIN85. shRNA constructs as well as an unspecific mock ${ }^{\text {sh }}$ control $^{2}$ were expressed via retroviral transduction. The knock down was applied to parental as well as to CD2AP-deficient cells (DT40 CD2AP ${ }^{-/}$), the CIN85 homolog. The cells were stimulated for various time points and cleared cellular lysates were prepared for immunoblotting. To show NF-KB activation, phosphorylation of $1 \mathrm{kB} \alpha$ was analyzed because it leads to the ubiquitinylation dependent degradation of IKBa followed by the release of the NF-KB subunits that can translocate to the nucleus and activate 
transcription. Probing the blot for $\mathrm{IKB}_{\mathrm{K}} \mathrm{p}$ phosphorylation revealed decreased phosphorylation in the parental $C I N 85^{\text {sh }}$ cells compared to the corresponding mock ${ }^{\text {sh }}$ control (figure 3.22.A). This finding is in contrast to the effect of CD2AP deficiency. In these cells, IKBa activation was increased compared to the parental mock ${ }^{\text {sh }}$ control cells. The shRNA mediated knock down of CIN85 in CD2AP-deficient cells (CD2AP ${ }^{-/}$CIN85 $\left.{ }^{\text {sh }}\right)$, however, showed the lowest phosphorylation signal for IKBa. These activation kinetics of $\mathrm{NF}-\mathrm{kB}$ were confirmed by the detection of IKKa/ $\beta$ phosphorylation, which constitutes the upstream kinase complex. The experiments showed that CIN85 is not just involved in NF$\mathrm{KB}$ activation in primary mouse and human B cells but also in the DT40 cell culture system. In addition, it indicates a function that is opposed to CD2AP in that context. For $\mathrm{Ca}^{2+}$ mobilization, CD2AP can compensate to some degree for the loss of CIN85 expression, whereas for BCR interaction or SLP65 protein stability CD2AP is dispensable. For NF-KB activation it has contrary, meaning inhibitory function.

To investigate the impact of CIN85 on the NF-kB pathway further, the human DG75 cell line system was used, which enables better comparison with the patient. However, in terms of this particular pathway, burkitt lymphoma cells are always preactived to some degree. On that account, NF-KB activation induced by BCR stimulation is hard to detect. To overcome this problem, I retrovirally transduced the protein kinase C $\beta$ II (PKC $\beta I I)$ in DG75 cells. PKC $\beta$ is known to be involved in BCR-mediated NF-KB activation (Su et al., 2002). In general both isoforms, $P K C \beta I$ and $P K C \beta I I$, are expressed in $B$ cells, however $P K C \beta I I$ is missing in DG75 B cells (figure 3.22.B).

Retroviral transduction of $\mathrm{PKC} \beta \mathrm{I}$ or PKC $\beta$ II was intended to enhance BCR-mediated NFKB activation. To test this, cleared cellular lysates from DG75 PKCßllwt and CIN85deficient PKCßIIwt DG75 cells were prepared and immunoblotted for phosphorylated

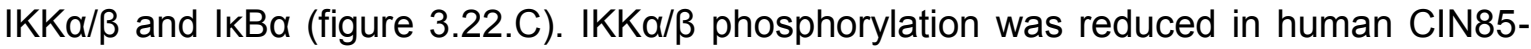
deficient DG75 cells and supports the findings of the chicken DT40 cell system. Phosphorylation of IKBa could not be used as readout for BCR-induced NF-KB activation in DG75 cells as it was phosphorylated in resting cells, too.

For membrane recruitment and BCR-mediated $\mathrm{Ca}^{2+}$ flux, only the full length CIN85 fulfills its function. In contrast, the N-terminal part, namely the three $\mathrm{SH} 3$ domains, is enough for SLP65 stabilization. To test which regions of CIN85 influence the process of NF-KB activation, CIN85-deficient DG75 cells expressing EGFP, CIN85wt, CIN85ASH3 or CIN85 $\triangle \mathrm{CC}$ were stimulated and cleared cellular lysates were prepared. The immunoblot stained for $\mathrm{plKK} \alpha / \beta$ showed approximately the same degree of NF-kB activation for the two truncated CIN85 variants if the signals for the Actin control were considered (figure 3.22.D). This activation was weaker than for the full length CIN85. The same was also true for again not inducible but steady state phosphorylation of $\mathrm{IKBa}$. This indicates that for 
positive CIN85 function in the NF-KB pathway the full length protein is necessary and truncated variants can only partly support activation.

A)
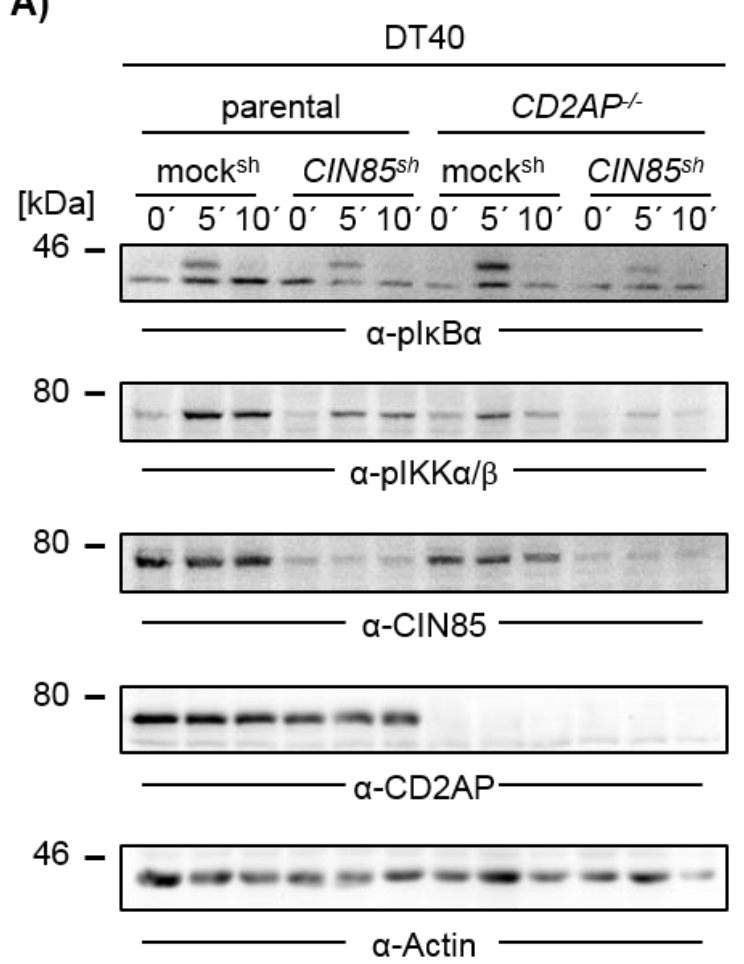

C)

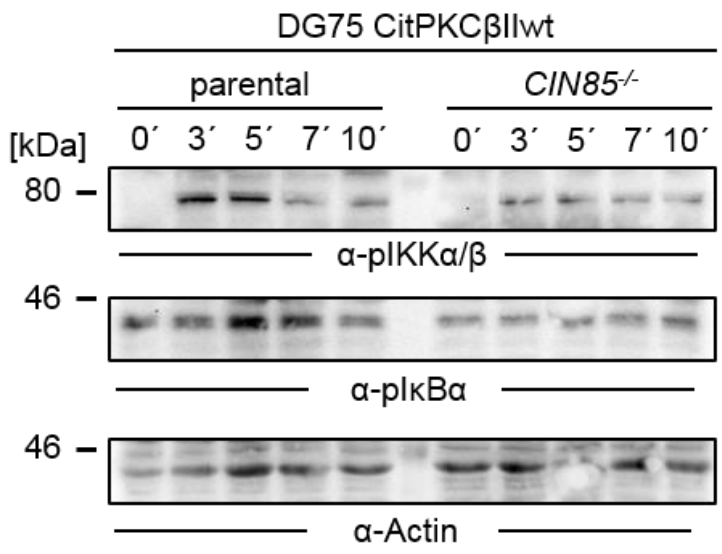

B) DG75 CIN85^

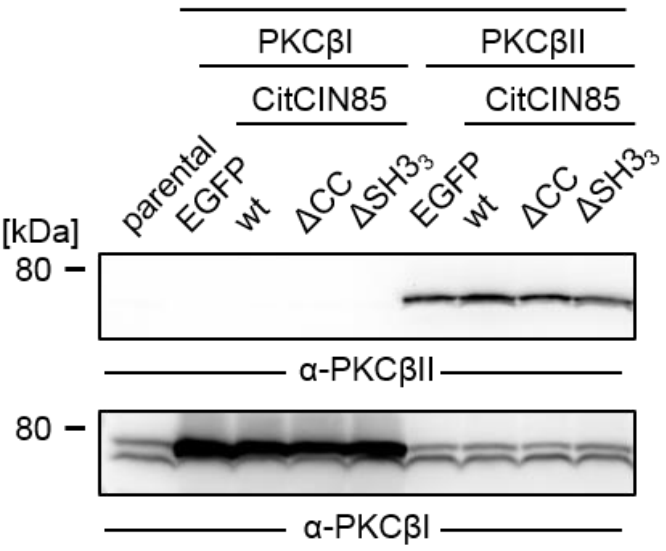

D) DG75 CIN85\% PKC 3 Ilwt

CitCIN85

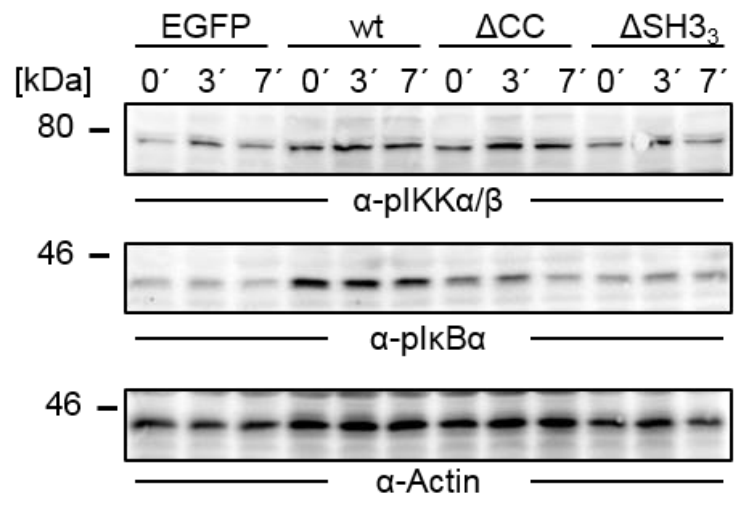

Figure 3.22: Loss of CIN85 diminishes BCR-induced NF-KB activation. A) DT40 parental and CD2APdeficient DT40 cells were retrovirally transduced with constructs coding for $\mathrm{CIN} 85^{\text {sh }}$ and mock $^{\text {sh }}$. These cells were left untreated or stimulated with $2 \mu \mathrm{g} / \mathrm{ml} \mathrm{M} 4$ for $5 \mathrm{~min}\left(5^{\prime}\right)$ and $10 \mathrm{~min}\left(10^{\prime}\right)$. Cleared cellular lysates were prepared, subjected to SDS-PAGE and the immunoblot was stained for plkBa, pIKK $\alpha / \beta$, CIN85, CD2AP and Actin. B) CIN85-deficient DG75 cells expressing EGFP, CitCIN85wt (wt), CitCIN85 $\triangle C C$ ( $\triangle C C$ ) or CitCIN85 $\triangle \mathrm{SH}_{3}\left(\triangle \mathrm{SH}_{3}\right)$ were retrovirally transduced with constructs for either $\mathrm{PKC} \beta \mathrm{I}$ or PKC $\beta$ II. Cleared cellular lysates of these cells and parental DG75 cells were prepared, subjected to SDS-PAGE and the immunoblot was stained with anti-PKC 3 II or anti-PKC $\beta I$ antibodies. C) DG75 and CIN85-deficient DG75 cells were retrovirally transduced with constructs coding for wild-type PKC $\beta I I$ (PKCßIIwt). These cells were left untreated or stimulated with $10 \mu \mathrm{g} / \mathrm{ml}$ anti-lgM $\mathrm{F}\left(\mathrm{ab}^{\prime}\right)_{2}$ fragments for $3 \min \left(3^{\prime}\right), 5 \mathrm{~min}\left(5^{\prime}\right), 7 \mathrm{~min}\left(7^{\prime}\right)$ and 10 $\min \left(10^{\prime}\right)$. Cleared cellular lysates were prepared, subjected to SDS-PAGE and the immunoblot was stained 


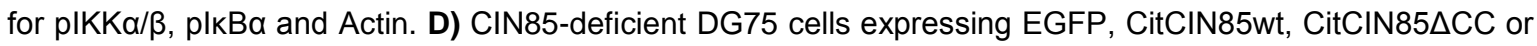
CitCIN85 $\triangle \mathrm{SH} 3$ were retrovirally transduced with constructs coding for wild-type PKCßII (PKCßIIwt). These cells were left untreated or stimulated with $10 \mu \mathrm{g} / \mathrm{ml}$ anti-lgM $\mathrm{F}\left(\mathrm{ab}^{\prime}\right)_{2}$ fragments for $3 \mathrm{~min}\left(3^{\prime}\right)$ and $7 \mathrm{~min}\left(7^{\prime}\right)$.

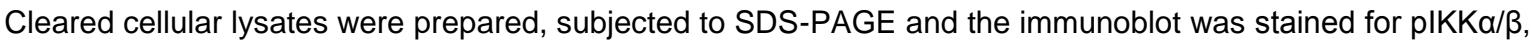
plкBa and Actin. The respective molecular weight of marker proteins (in $\mathrm{kDa}$ ) is indicated on the left.

\subsubsection{CIN85 associates with the NF-KB activator IKKa}

The activating CIN85 impact on NF-kB signaling is shown but it is still unknown at which site in the signaling cascade CIN85 is involved and how it functions in this context. To elucidate this further, GST-CIN85 affinity purification experiments in resting or BCRstimulated CIN85-deficient DG75 cells were performed using GST-only as control. Immunoblotting with antibodies against known NF-KB pathway proteins showed an interaction of CIN85 with the kinase IKKa (figure 3.23.A). Also for CARMA1 weak interaction with CIN85 was detected. This might indicate that the adaptor protein CIN85 is part of a big NF-KB signaling protein complex.
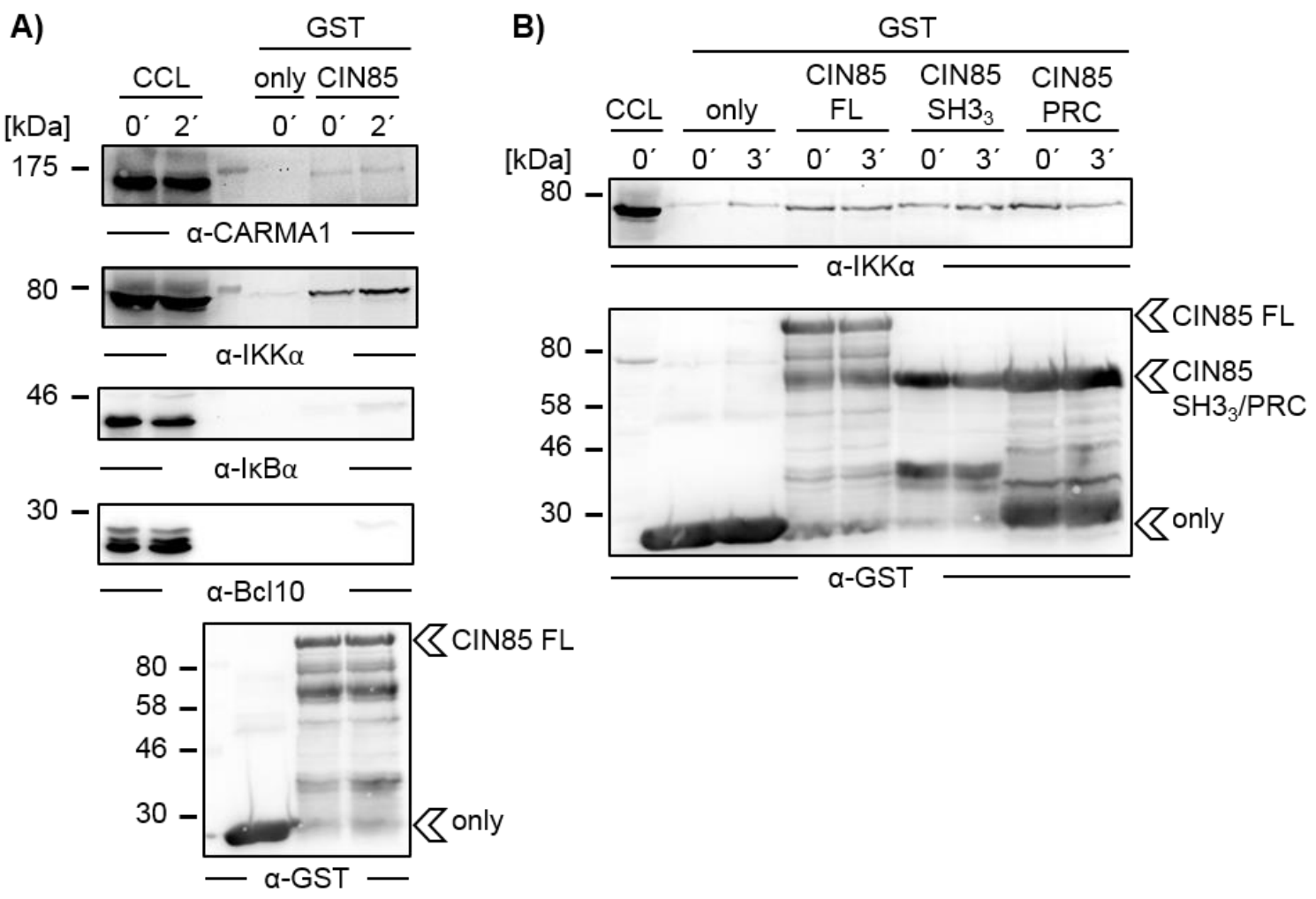

Figure 3.23: CIN85 shows interaction with IKKa. A) Cleared cellular lysates of resting CIN85-deficient DG75 or with $10 \mu \mathrm{g} / \mathrm{ml}$ anti-lgM $\mathrm{F}\left(\mathrm{ab}^{\prime}\right)_{2}$ fragments stimulated cells were directly subjected to SDS-PAGE or further used for affinity purification with GST-only and GST-CIN85 full length (FL). The immunoblot was stained with antibodies detecting CARMA1, IKKa, IKBa, Bcl10 and GST. B) Cleared cellular lysates of resting CIN85-deficient DG75 or stimulated with $10 \mu \mathrm{g} / \mathrm{ml}$ anti-lgM $\mathrm{F}\left(a b^{\prime}\right)_{2}$ fragments were directly subjected to SDS- 
PAGE or further used for affinity purification with GST-only, GST-CIN85 full length (FL), GST-CIN85SH3 3 or GST-CIN85PRC. The immunoblot was stained with antibodies detecting IKKa and GST. The respective molecular weight of marker proteins (in $\mathrm{kDa}$ ) is indicated on the left.

Further evidence for this hypothesis was obtained by affinity purification experiments using full length $\mathrm{CIN} 85(\mathrm{FL})$, truncated $\mathrm{N}$-terminal $\left(\mathrm{SH}_{3}\right)$ or $\mathrm{C}$-terminal $(\mathrm{PRC})$ variants as GST-fusion proteins. Like shown in figure 3.23.B, the strongest interaction with IKKa was obtained for the full length CIN85 fusion protein. Both truncated CIN85 variants revealed weaker interaction with IKK compared to full length CIN85. The strength of the CIN85IKKa interaction is an indication for CIN85 not being a direct interaction partner of IKKa but rather being part of the same protein complex in resting as well BCR-activated DG75 cells.

\subsubsection{CIN85 interacts stimulation dependent with $\mathrm{PKC} \beta$ and influences its localization in DG75 cells}

To test CIN85 interaction with another known NF-KB signaling component, PKC $\beta$ affinity purification experiments were performed using GST-CIN85 fusion proteins. Therefore, CIN85-deficient DG75 cells were retrovirally transduced with a construct encoding citrinetagged wild-type PKC $\beta$ II. Since PKC $\beta I$ is otherwise not expressed in DG75 cells, these cells constitute an optimal model system to investigate a potential CIN85-PKC $\beta$ II interaction. In the ectopically expressed construct, the tag is located at the $\mathrm{N}$-terminal part of PKC $\beta I I$ and hence shields the pseudosubstrate domain (figure 3.24.A). This results in a constantly active state of the protein as the pseudosubstrate cannot bind to the kinase domain to prevent PKC 3 II activation. Affinity purification experiments with GST-CIN85FL, $\mathrm{GST}-\mathrm{CIN85SH}_{3}$ or GST-CIN85PRC revealed strongest interaction with PKC $\beta I$ and CIN85FL. In contrast, only weak interaction was detected between PKCßII and the CIN85 SH3 domains, whereas the PRC region showed no interaction at all (figure 3.24.B).

Another experimental setup using GST-only or GST-CIN85SH3 3 for affinity purification in resting, 2 or 5 min activated cells showed that the CIN85-PKC $\beta$ interaction was dependent on BCR stimulation (figure 3.24.C). In resting cells, this interaction was visible but gone after 2 min of stimulation. Since in unstimulated cells the N-terminal citrine-tagged PKC $B$ II is thought to be active, it might be that upon BCR stimulation the construct transiently changes into its inactive form given that an extended stimulation period of 5 min restored this signal. Based on these results, the hypothesis arose that a serine, threonine, tyrosine or histidine residue in close proximity to the CIN85 interaction site might be phosphorylated upon BCR stimulation. Hence, interaction could be prevented by 
phosphorylation of the respective residues. Screening of the PKC $\beta$ amino acid sequence for putative SH3 binding motifs identified the seven amino acid stretch PASDDPR (aa 9399) (figure 3.24.D).

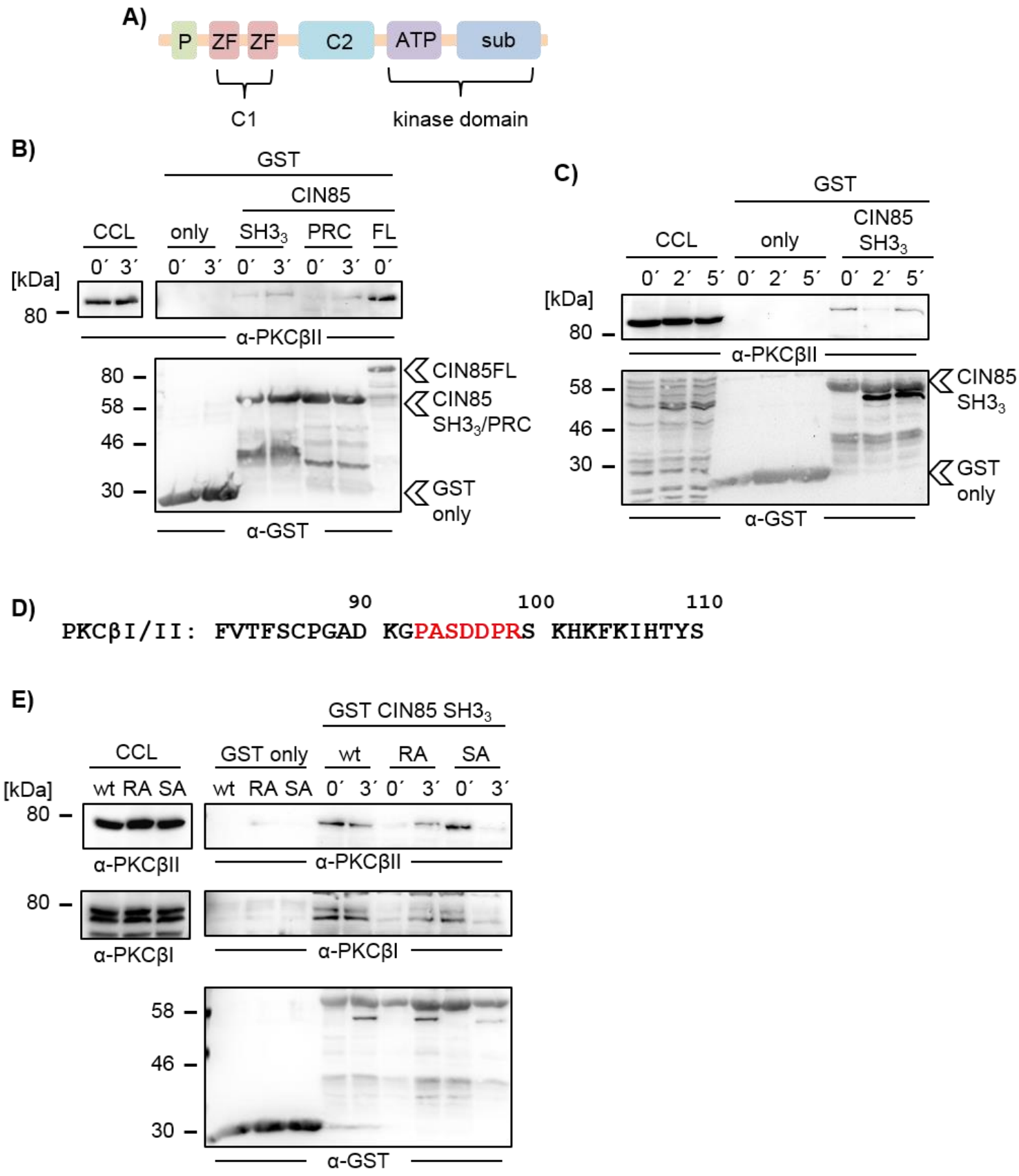

Figure 3.24: PKC $\beta$ II interacts with CIN85 upon BCR stimulation. A) PKC $\beta$ domain architecture. At the Nterminus a pseudosubstrate $(P)$ is located that binds to and thereby shields the kinase domain from activation. This is followed by two zinc finger motifs (ZF), which depict a $\mathrm{C} 1$ domain and promote binding to the second messenger diacylglycerol. The $\mathrm{C} 1$ domain is followed by a $\mathrm{C} 2$ domain, which promotes membrane binding. At the C-terminus the catalytic domain that contains an ATP and a substrate binding part (sub) completes the protein. B) CIN85-deficient DG75 cells were retrovirally transduced with constructs encoding citrine-tagged wild-type PKCßII. Cleared cellular lysates of resting or BCR stimulated DG75 cells $\left(10 \mu \mathrm{g} / \mathrm{ml}\right.$ anti-lgM F $\left(\mathrm{ab}^{\prime}\right)_{2}$ fragments) were directly subjected to SDS-PAGE or used for affinity purification experiments with GST-only, 
GST-CIN85FL, GST-CIN85SH3 3 or GST-CIN85PRC. The immunoblot was stained with antibodies detecting PKC 3 II or GST. C) Cleared cellular lysates of resting or BCR stimulated ( $10 \mu \mathrm{g} / \mathrm{ml}$ anti-lgM F(ab' $)_{2}$ fragments) cells described in B) were directly prepared and subjected to SDS-PAGE or further used for affinity purification experiments with GST-only or GST-CIN85SH3 3 . The immunoblot was stained with antibodies detecting PKCßII or GST. D) The PKCßI/II amino acid sequence from position 80-110 highlighting a putative interaction site of CIN85 in red. E) CIN85-deficient DG75 cells were retrovirally transduced with constructs encoding wildtype PKCßII (wt), PKCßII R99A or PKC $/$ II S100A. Cleared cellular lysates of resting or BCR stimulated DG75 cells $\left(10 \mu \mathrm{g} / \mathrm{ml}\right.$ anti-lgM $\mathrm{F}\left(\mathrm{ab} \mathrm{b}_{2}\right)_{2}$ fragments) were directly subjected to SDS-PAGE or used for affinity purification experiments with GST-only or GST-CIN85SH3 3 . The immunoblot was stained with antibodies detecting PKC $\beta$ II, PKC $\beta$ I or GST. The respective molecular weight of marker proteins (in $\mathrm{kDa}$ ) is indicated on the left.

Right behind this sequence a serine is present (aa 100), which could be a potential site of phosphorylation. To test this hypothesis, the serine was changed via site directed mutagenesis to an alanine (S100A). After retroviral transduction of CIN85-deficient cells with this PKC $\beta$ construct, affinity purification experiments were performed using GST$\mathrm{CIN85SH}_{3}$ or GST-only. Probing the immunoblot for PKC 3 IIS100A showed interaction with CIN85 in resting but not stimulated cells (figure 3.24.E). As a control, the blot was probed with anti-PKC $\beta I$ antibodies, which revealed the same kinetic of signal strength. Since PKC $\beta I$ is endogenously expressed and still contains a serine at this position, binding differences did not depend on the serine 100.

In addition, the preceding proline-arginine motif was tested, whether it is the site of interaction with CIN85. This proline-arginine motif does not resemble a complete CIN85 interaction site compared to the one in SLP65 (PxPxPR). It was used for site directed mutagenesis changing PASDDPR to PASDDPA (R99A). This construct was expressed in CIN85-deficient DG75 cells (CIN85/ PKC 3 IIR99A) and affinity purification experiments using GST-CIN85SH3 3 or GST-only were performed. The results show less binding for PKC $\beta I I$ in resting cells, however, this was also the case for endogenous wild-type PKC $\beta I$ (figure 3.24.E). This indicates that R99 is not involved in CIN85 interaction. As the interaction between CIN85 and PKC 3 II was quite weak, it might be that this interaction is not due to direct binding.

\subsubsection{Plasma membrane localization of PKC $\beta$ II is regulated by CIN85}

Expression of N-terminally citrine-tagged wild-type PKC 3 II in parental DG75 and CIN85deficient cells allowed for visualizing of the protein within the cell with confocal laser scanning microscopy. Localization of PKC $\beta I I$ is supposed to be in the cytoplasm in resting $B$ cells, followed by translocation to the plasma membrane upon BCR stimulation (Su et al., 2002). In the here performed experiment, constitutively active $\mathrm{N}$-terminally citrine- 
tagged PKC $\beta$ II was prerecruited to the membrane in approximately $70 \%$ of the citrinepositive parental DG75 cells (figure 3.25.A, left panel). This was in contrast to the location of PKC $\beta I I$ in resting CIN85-deficient DG75 cells. Here, PKC $\beta I I$ was trapped in the cytoplasm (figure 3.25.A, right panel) which implicates a role for CIN85 in the localization of PKCßII although the interaction is likely to be indirect.

Furthermore, CIN85 impact on the localization of other known NF-KB signaling proteins which act downstream of $\mathrm{PKC} \beta \|$ and hence might be present in the same signaling complexes was analysed. Citrine-tagged variants of CARMA1 and Bcl10, which are members of the so called CBM complex, were analyzed as these proteins are direct targets of PKC $\beta$ and constitute a critical role in the activation of NF-KB upon BCR stimulation (Rawlings et al., 2006; Schulze-Luehrmann and Ghosh, 2006).

A)
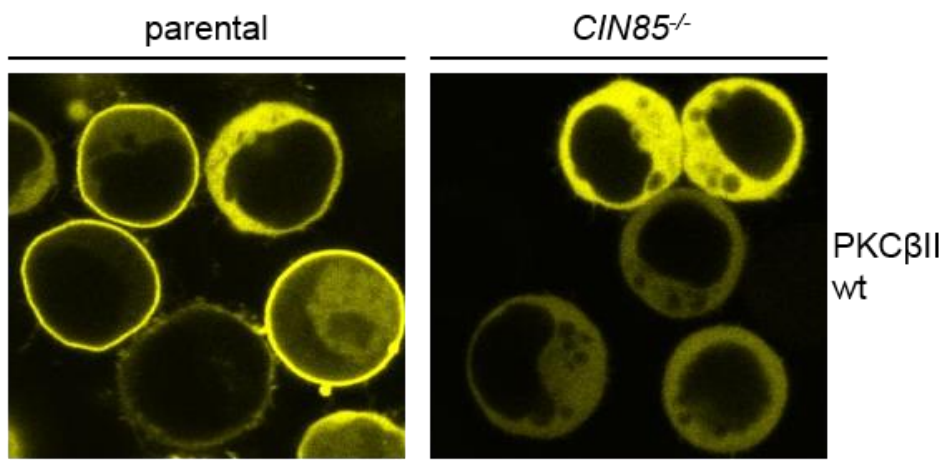

B)
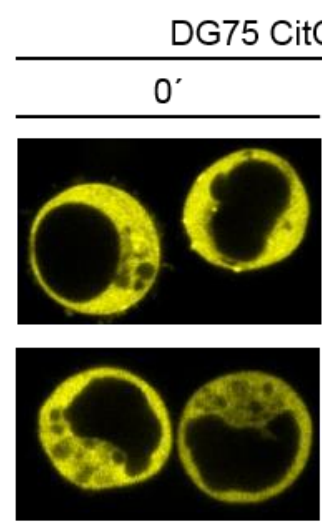

C)

$\frac{\text { DG75 CitBcl10wt }}{0^{\prime}}$

parental
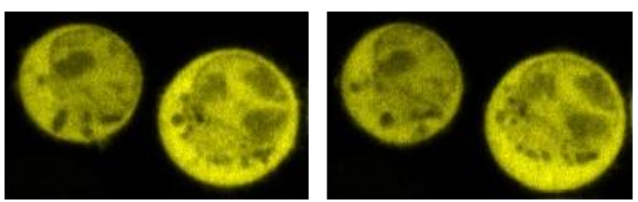

CIN85-
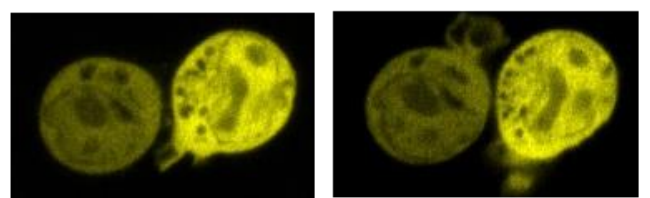

Figure 3.25: CIN85 affects localization of PKC $\beta$ II but not of CARMA1 and Bcl10. A) Parental DG75 and CIN85-deficient DG75 cells were retrovirally transduced with constructs coding for citrine-tagged wild-type PKCßII (PKCßII wt) and subjected to laser scanning microscopy without stimulation. DG75 and CIN85deficient DG75 cells were retrovirally transduced with constructs coding for citrine-tagged wild-type CARMA1 (CitCARMA1wt) (B) and citrine-tagged wild-type Bcl10 (CitBcl10wt) (C). The localization of tagged proteins was analyzed in unstimulated or stimulated $\left(10 \mu \mathrm{g} / \mathrm{ml} \text { anti-lgM } \mathrm{F}(\mathrm{ab})_{2}\right)_{2}$ fragments) DG75 cells by confocal laser scanning microscopy. 
CARMA1 was located in the cytoplasm of parental DG75 as well as CIN85-deficient DG75 cells (figure 3.25.B). Upon antigen-receptor stimulation, it was not recruited to the plasma membrane in DG75 cells although this was shown by Wang and colleagues in T cells (Wang et al., 2004). Also additionally expressed Bcl10 was not recruited in parental and CIN85-deficient DG75 cells upon BCR stimulation (figure 3.25.C). A reason for this might be the experimental setup in which CARMA1 and BCL10 were additionally expressed to the endogenous proteins, which might interfere with the ectopically expressed citrinetagged variants. Nevertheless, the location of both proteins did not differ between parental and CIN85-deficient DG75 cells. Therefore, I assume that CIN85 in particular regulates the localization of PKC $\beta I I$ and does not influence the other here tested NF-KB linked proteins.

\subsubsection{CIN85 binds to Rhotekin2 (RTKN2) with its SH3 domains}

Mass spectrometry analyses of strep-tagged purified CIN85 in chicken DT40 as well as human burkitt lymphoma Ramos cells by Julius Kühn in cooperation with the group of Prof. Henning Urlaub at the Max Planck Institute for biophysical chemistry in Göttingen revealed a new interaction partner of CIN85, namely RTKN2 (Kühn, 2015). There is only little known about this protein but it is described to have an activatory function in NF-KB signaling (Collier et al., 2009; Myouzen et al., 2012). At first, the binding of RTKN2 to CIN85 was tested by affinity purification experiments using GST-CIN85 full length as well as truncated variants (figure 3.26.A). Strong interaction was detected for the full length CIN85 as well as the three $\mathrm{SH} 3$ domains. This implicates binding of the CIN85 SH3 domains to a proline-arginine motif in RTKN2. A closer look at the amino acid sequence of human RTKN2 revealed two binding motifs for CIN85 whereas chicken RTKN2 only harbors one motif (figure 3.26.C).

To test whether all three SH3 domains are capable to interact with RTKN2, affinity purifications were performed using GST-CIN85SH3 A, B, C or GST-only (figure 3.26.B). The immunoblot probed with anti-RTKN2 antibodies showed equal binding of all three SH3 domains. The next step was to test whether the two described putative atypical proline-arginine motifs are the sites of CIN85 binding. Therefore, proline to arginine substitutions at positions 560 or 591 in citrine-tagged RTKN2 were introduced. These RTKN2 variants or wild-type RTKN2 were expressed in CIN85-deficient DG75 cells (figure 3.26.D) and used in affinity purification experiments with GST-CIN85SH3 3 or GST-only. The immunoblot was probed with anti-GFP antibodies to exclusively detect ectopically expressed RTKN2 variants and not the endogenous protein (figure 3.26.E). Neither substitutions of R560 nor R591 prevented or even weakened the interaction with CIN85. A 
possible explanation is that as long as one of the two motifs is functional it can take over for the other one. To confirm this hypothesis, mutagenesis of both motifs in one construct has to be done.

A)

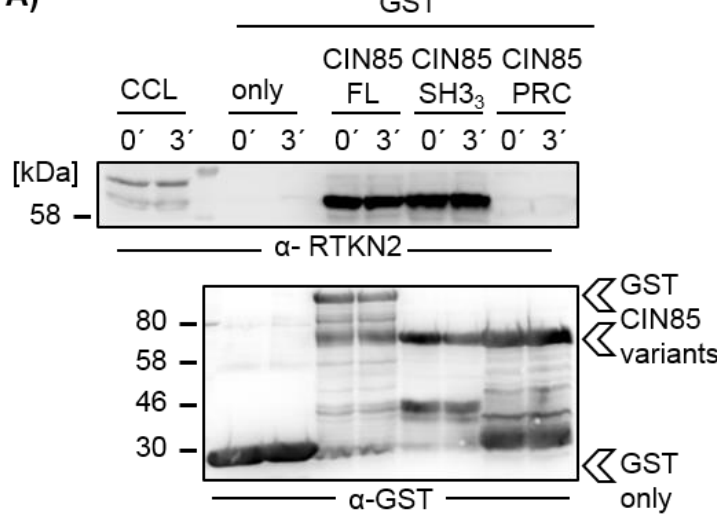

B)

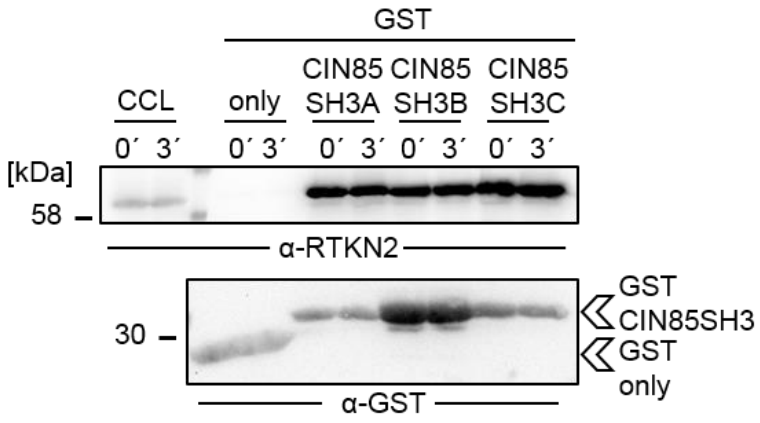

580

$590-600$

$\begin{array}{ll}\text { human: } & \text { HLQKPMAAPR KLLPARRNRL SDGEHTDTKT NFEAKPVPAP RQKSIKDILD } \\ \text { chicken: } & \text { IAAPHHICPC RKNSLADHKN PISLVTKTES ETKPVPTPRO KRITEVLDPR }\end{array}$

D)

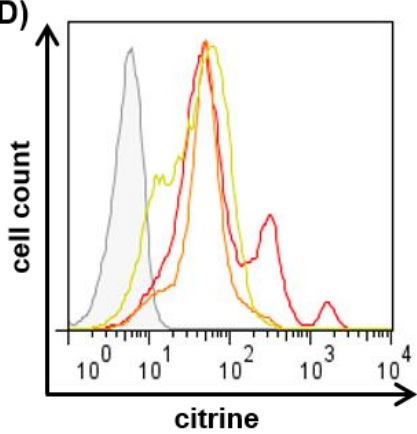

DG75

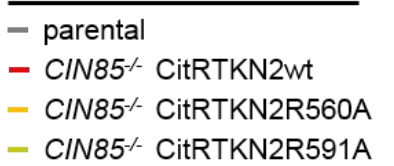

E)
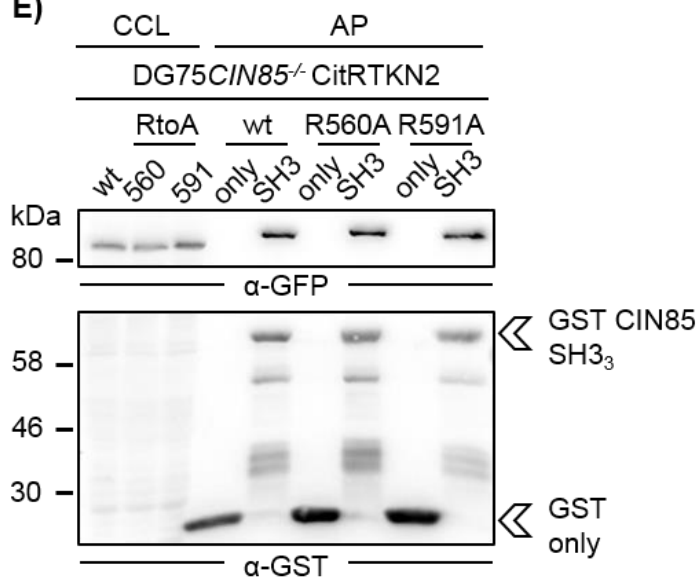

Figure 3.26: RTKN2 is a constitutive ligand of CIN85. A) Cleared cellular lysates of resting or BCR stimulated with $10 \mu \mathrm{g} / \mathrm{ml}$ anti-IgM $F\left(a b^{\prime}\right)_{2}$ fragments DG75 cells were directly subjected to SDS-PAGE or used for affinity purification experiments using GST-only and GST-CIN85FL, GST-CIN85SH33, GST-CIN85PRC. The immunoblot was stained with antibodies detecting RTKN2 or GST. B) Cleared cellular lysates of resting or with $10 \mu \mathrm{g} / \mathrm{ml}$ anti-IgM $F\left(a b^{\prime}\right)_{2}$ fragments stimulated DG75 cells were either subjected directly to SDS-PAGE or used for affinity purification experiments using GST-only or GST-CIN85SH3 A, B or C. The immunoblot was stained with antibodies detecting RTKN2 or GST. C) Detail of the human and chicken RTKN2 amino acid sequences from position 550-600 including the putative interaction site of CIN85 in red. D) CIN85-deficient DG75 cells were retrovirally transduced with constructs coding for wild-type citrine-tagged RTKN2 (CitRTKN2wt), RTKN2 R560A (CitRTKN2R560A) or RTKN2 R591A (CitRTKN2R591A). The expression levels were analyzed by flow cytometry. E) Cleared cellular lysates of the cells described in C) were either directly subjected to SDS-PAGE or used in affinity purification experiments using GST-only or GST-CIN85SH33. The 
immunoblot was stained with antibodies detecting RTKN2 or GST. The respective molecular weight of marker proteins (in $\mathrm{kDa}$ ) is indicated on the left.

3.6.6 Chicken RTKN2 acts as activating regulator of $\mathrm{Ca}^{2+}$ and NF-KB signaling in B cells

As interaction of CIN85 and RTKN2 could be shown I tested RTKN2 involvement in NF-KB activation in our cell line system. To this end, shRNA-mediated knock down of RTKN2 was performed in the chicken B cell line DT40, which was chosen, since here NF-KB activation upon $\mathrm{BCR}$ stimulation could be analyzed solidly via preparation of cleared cellular lysates followed by immunoblotting. Both shRNA constructs reduced RTKN2 expression up to $60-70 \%$ compared to the shRNA mock control transduced DT40 cells (figure 3.27.A). Reduction of RTKN2 protein levels were checked for each experiment. 

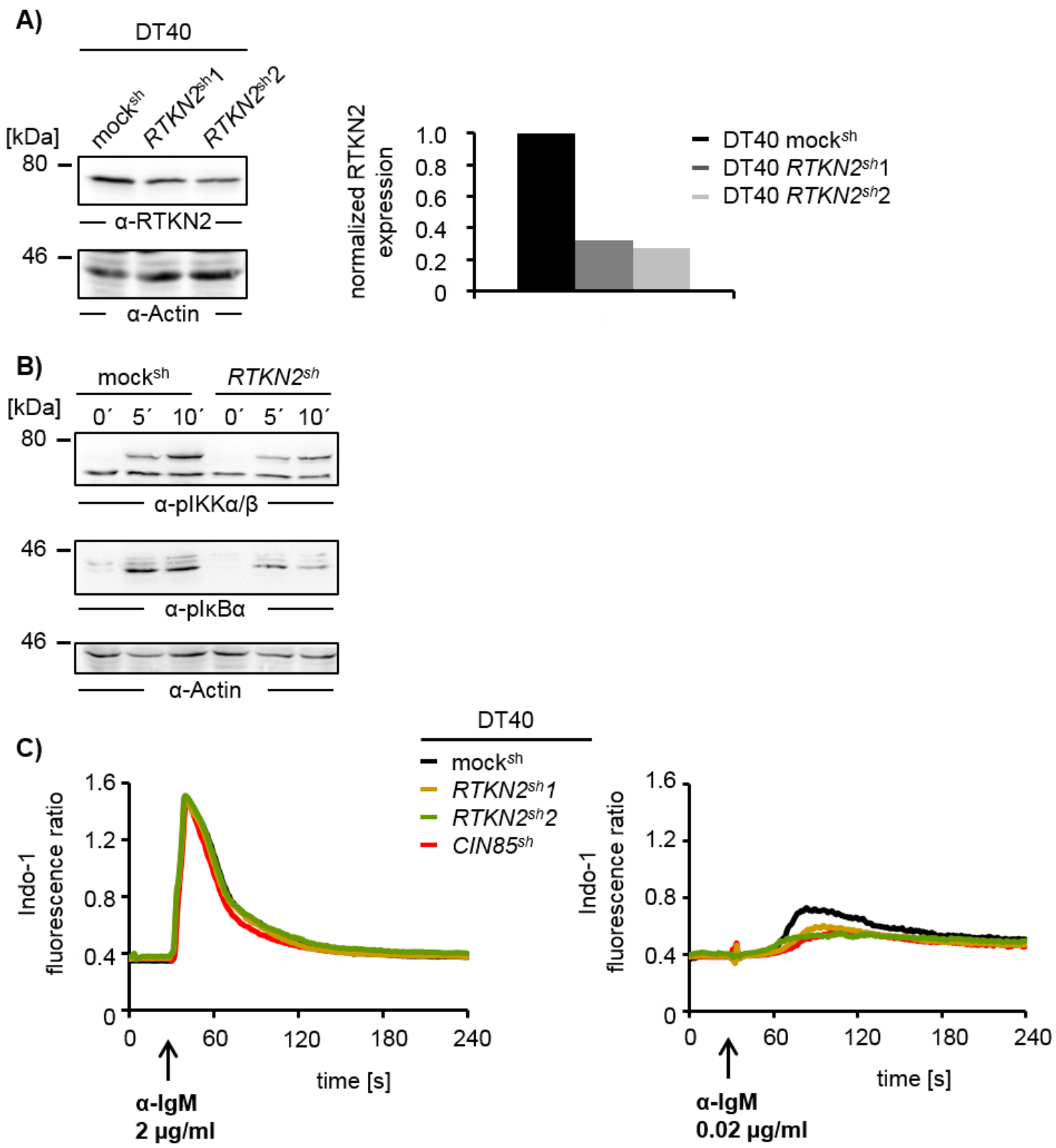

Figure 3.27: Reduced RTKN2 protein levels led to diminished NF-KB and $\mathrm{Ca}^{2+}$ signaling in DT40 cells. A) DT40 cells were retrovirally transduced with two different shRNAs targeting RTKN2 mRNA (RTKN2 ${ }^{\text {sh }} 1 \& 2$ ) or an unspecific mock control (mock ${ }^{\text {sh }}$ ). Cleared cellular lysates of these cells were prepared, subjected to SDS-PAGE and the blot was probed for RTKN2 and Actin (left panel). Quantification of the RTKN2 protein level compared to Actin as loading control. Resulting relative RTKN2 protein amounts of the different RTKN2 shRNA constructs (RTKN $\left.2^{\text {sh }} 1 \& 2\right)$ were normalized to the shRNA mock control (mock $\left.{ }^{\text {sh }}\right)$. B) The cells described in A) were stimulated with $2 \mu \mathrm{g} / \mathrm{ml} \mathrm{M}$, cleared cellular lysates were prepared and subjected to immunoblotting. The blot was stained for phosphorylation of IKK $\alpha / \beta(p \mid K K \alpha / \beta)$ and IKBa $(p \mid K B \alpha)$, Actin served as loading control. The respective molecular weight of marker proteins (in $\mathrm{kDa}$ ) is indicated on the left. C) The cells described in A) were analyzed for $\mathrm{Ca}^{2+}$ mobilization upon BCR stimulation with either $2 \mu \mathrm{g} / \mathrm{ml}$ (left graph) or $0.02 \mu \mathrm{g} / \mathrm{ml} \mathrm{M} 4$ (right graph) as before. For comparison, the shRNA-mediated knock down of CIN85 (CIN85 ${ }^{\text {sh }}$ ) was included. 
NF-KB activation was induced by BCR simulation with anti-chicken IgM (M4), cleared cellular lysates were prepared and the immunoblot was stained for $1 \mathrm{KK \alpha} / \beta$ and $1 \mathrm{kB \alpha}$ phosphorylation (figure 3.27.B). These two activation markers of the NF-kB pathway showed both reduced phosphorylation upon BCR stimulation in the DT40 RTKN2 ${ }^{\text {sh }}$ cells compared to the mock control. This indicates an activating function for RTKN2 in the process of NF-KB activation after BCR stimulation in chicken DT40 cells.

Furthermore, RTKN2 involvement in BCR-mediated $\mathrm{Ca}^{2+}$ flux was analyzed in these cells (figure 3.27.C). After BCR stimulation with $2 \mu \mathrm{g} / \mathrm{ml} \mathrm{M} 4, \mathrm{Ca}^{2+}$ profiles were identical for cells targeted by RTKN2 shRNA constructs and the mock control. With decreased antibody amounts of $0.02 \mu \mathrm{g} / \mathrm{ml} \mathrm{M} 4$, reduced $\mathrm{Ca}^{2+}$ mobilization was detected for RTKN2 knock down compared to the mock ${ }^{\text {sh }}$ control. The decreased $\mathrm{Ca}^{2+}$ flux was comparable

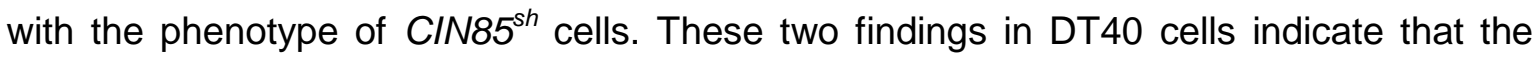
interaction partners CIN85 and RTKN2 have a similar input in the here analyzed cellular functions of $\mathrm{Ca}^{2+}$ mobilization and NF-KB activation.

3.6.7 RTKN2 neither acts in $\mathrm{Ca}^{2+}$ mobilization nor in NF-KB activation in human $\mathrm{B}$ cells

To test whether RTKN2 has the same function in human cells, shRNA-mediated RTKN2 knock down was performed in DG75 cells. Chicken and human RTKN2 proteins share $64.7 \%$ homology based on EMBOSS Needle alignment. The shRNA mediated efficiency of RTKN2 protein reduction was up to $90 \%$ compared to the mock ${ }^{\text {sh }}$ control based on immunoblotting (figure 3.28.A). Analysis of NF-KB activation revealed that stimulation of the $\mathrm{BCR}$ mediated similar IKKa/ $\beta$ phosphorylation in mock ${ }^{\text {sh }}$ and $R T K N 2^{\text {sh }}$ transduced DG75 cells shown in figure 3.28.B. Additionally, the non-inducible IKBa phosphorylation in DG75 showed equal signal strength. In the human B cell line DG75, reduction of RTKN2 protein levels, although more effective compared to DT40, did not affect the NF-KB pathway. 
A) DG75

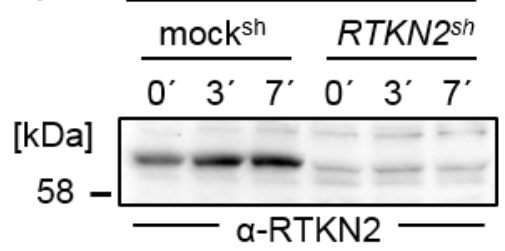

46
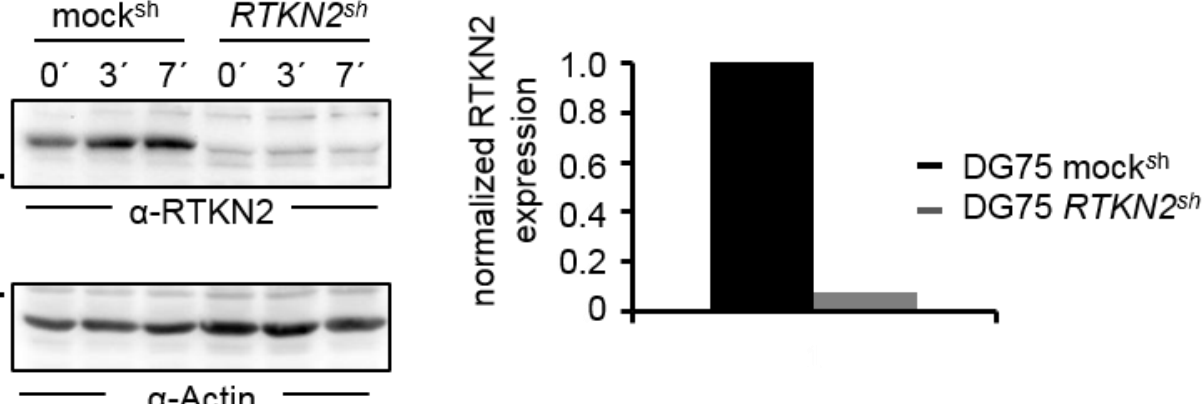

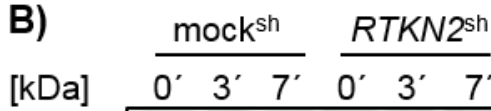

C)

parental

CIN85-
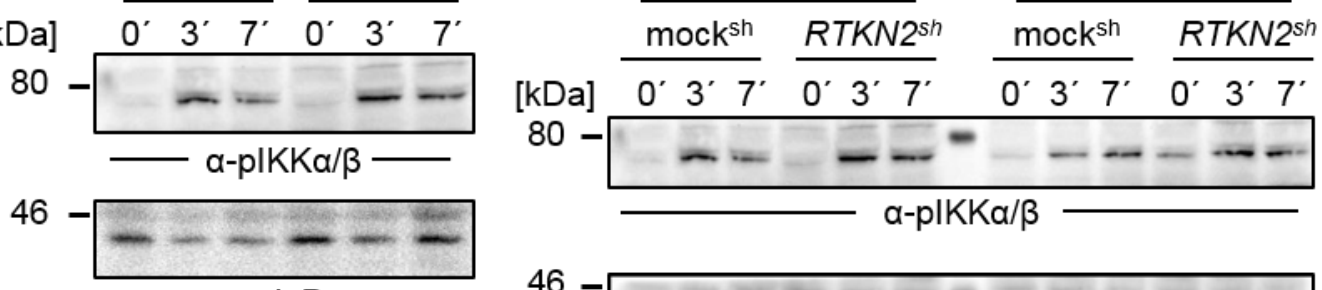

46
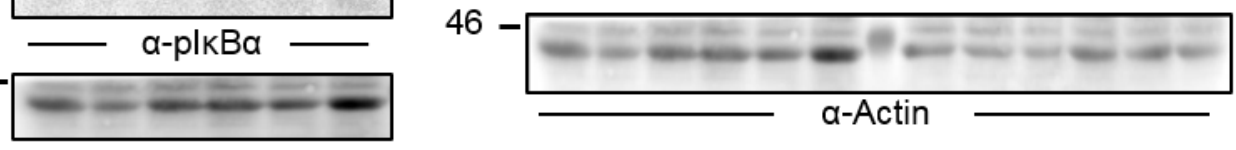

D)
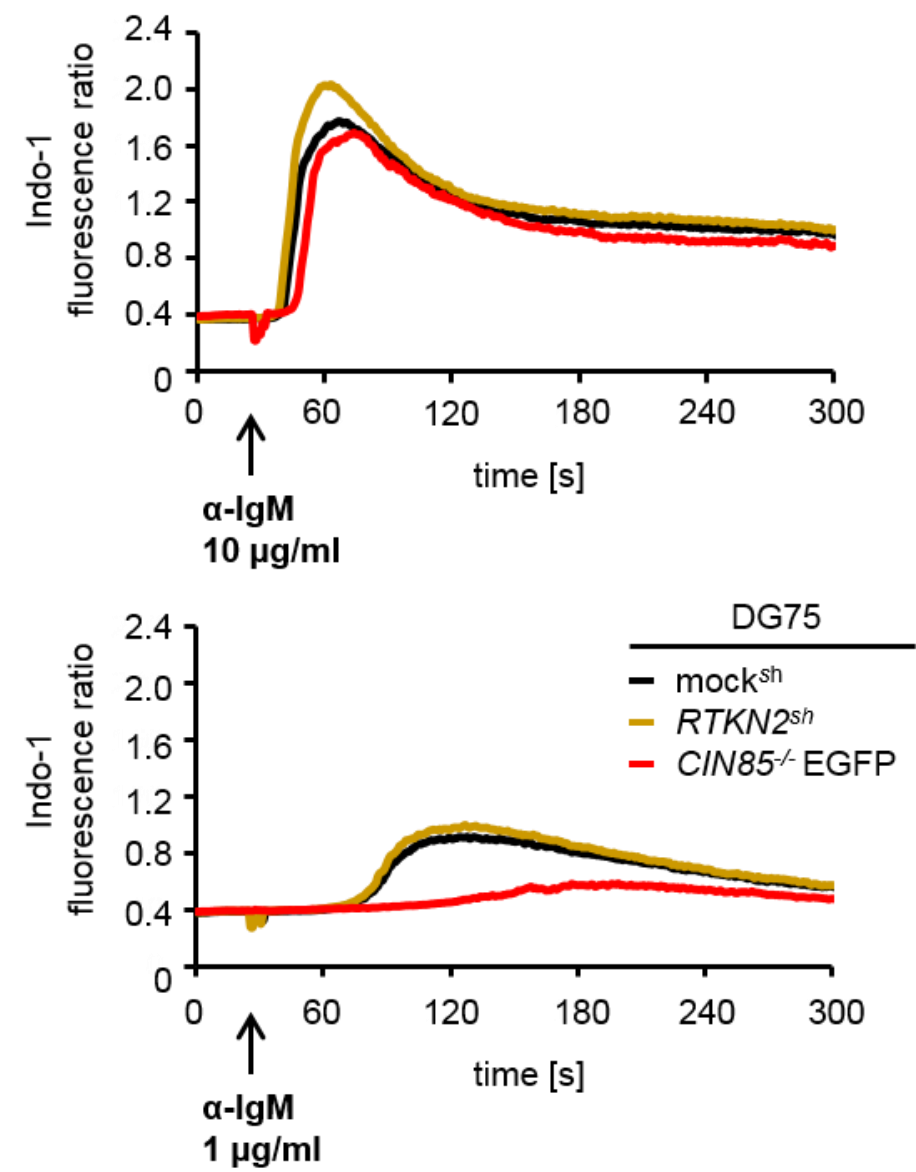
Figure 3.28: Reduced RTKN2 protein levels in DG75 cells did not affect $\mathrm{Ca}^{2+}$ and NF-KB signaling. A) DG75 cells were retrovirally transduced with shRNA targeting RTKN2 mRNA (RTKN2 $\left.{ }^{\text {sh }}\right)$ or an unspecific mock control $\left(\mathrm{mock}^{\text {sh }}\right)$. Cleared cellular lysates of these cells were prepared, subjected to SDS-PAGE and the blot was probed for RTKN2 and Actin (left panel). Quantification of the RTKN2 protein levels compared to Actin as loading control (right panel). Resulting relative RTKN2 protein amount in RTKN2 shRNA ( $R T K N 2^{\text {sh }}$ ) cells was normalized to the shRNA mock control $\left(\mathrm{mock}^{\mathrm{sh}}\right)$. B) The cells described in A) were left unstimulated or stimulated with $10 \mu \mathrm{g} / \mathrm{ml}$ anti-lgM $F\left(a b^{\prime}\right)_{2}$ fragments for $3\left(3^{\prime}\right)$ min and $7 \mathrm{~min}\left(7^{\prime}\right)$, cleared cellular lysates were prepared and subjected to immunoblotting. The blot was stained for phosphorylation of $I K K \alpha / \beta(p / K K \alpha / \beta)$ and IKBa (p|kBa), Actin served as loading control. The respective molecular weight of marker proteins (in $\mathrm{kDa}$ ) is indicated on the left. C) The cells described in A) and RTKN2 shRNA targeted CIN85-deficient DG75 cells were left unstimulated or stimulated with $10 \mu \mathrm{g} / \mathrm{ml}$ anti-IgM $\mathrm{F}\left(\mathrm{ab}^{\prime}\right)_{2}$ fragments for $3\left(3^{\prime}\right) \min$ and $7 \mathrm{~min}\left(7^{\prime}\right)$, cleared cellular lysates were prepared and subjected to immunoblotting. The blot was stained for phosphorylation of IKKa/ $/(\mathrm{plKK} / \beta)$, Actin served as loading control. The respective molecular weight of marker proteins (in $\mathrm{kDa}$ ) is indicated on the left. D) The cells described in A) were analyzed for $\mathrm{Ca}^{2+}$ mobilization upon BCR stimulation with either $10 \mu \mathrm{g} / \mathrm{ml}$ (upper graph) or $1 \mu \mathrm{g} / \mathrm{ml}$ anti-lgM $\mathrm{F}\left(\mathrm{ab}^{\prime}\right)_{2}$ fragments (lower graph) as before. For comparison, CIN85-deficient cells expressing EGFP were included.

To see whether RTKN2 might have an additional influence in CIN85-deficient DG75 cells, a RTKN2 knock down was performed in parental and CIN85-deficient cells (figure 3.28.C). Also in this experimental setup, the knock down of RTKN2 did not influence IKKa/ $\beta$ phosphorylation.

Figure 3.28.D shows $\mathrm{Ca}^{2+}$ signaling in RTKN2 knock down cells. For comparison of the $\mathrm{Ca}^{2+}$ phenotype, CIN85-deficient cells were included in the measurement. Stimulation of the human BCR with $10 \mu \mathrm{g} / \mathrm{ml}$ anti-IgM $\mathrm{F}\left(\mathrm{ab}^{\prime}\right)_{2}$ fragments did induce similar $\mathrm{Ca}^{2+}$ profiles in the analyzed cells. When the amount of stimulating antibody was reduced to a more physiological state of B cell activation, the RTKN2 knock down in human DG75 did in contrast to chicken DT40 cells not interfere with BCR-induced $\mathrm{Ca}^{2+}$ signaling.

\subsubsection{Protein stability of the ubiquitously localized RTKN2 depends on CIN85}

For additional analyses of RTKN2, it was ectopically expressed with a citrine-tag to allow for visualizing by confocal laser scanning microscopy. After viral transduction of this construct in DG75 parental (DG75 RTKN2wt) and CIN85-deficient cells (CIN85'RTKN2wt) they were sorted to gain equal citrine expression (figure 3.29.A). Confocal analyses showed location of RTKN2 in the cytoplasm and the nucleus (figure 3.29.B). Stimulation via the BCR did not induce RTKN2 membrane translocation, which might be due to endogenous protein levels. Nevertheless, the results indicate that the localization of RTKN2 is independent of CIN85 expression as it was unaltered between parental and CIN85-deficient DG75 cells. 
Furthermore, the influence of CIN85 as a ligand in the context of RTKN2 stability was analyzed, since this interaction is like already shown for SLP65 (compare chapter 3.4) also mediated by the SH3 domains of CIN85. To this end, cleared cellular lysates of CIN85-deficient DG75 cells expressing EGFP, CIN85wt, $\Delta \mathrm{CC}$ or $\Delta \mathrm{SH}_{3}$ were prepared and the immunoblot was probed for RTKN2 and Actin (figure 3.29.C). The results show that reduced RTKN2 protein levels in CIN85-deficient DG75 cells can be restored by the expression of either CIN85 full length or CIN85 $\Delta$ CC. These findings corresponded with the stabilizing role of CIN85 SH3 domains for SLP65 protein levels.

A)
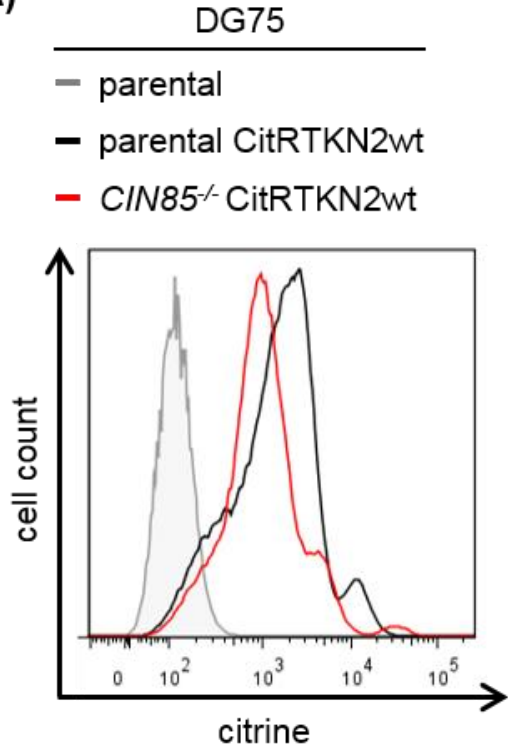

B)

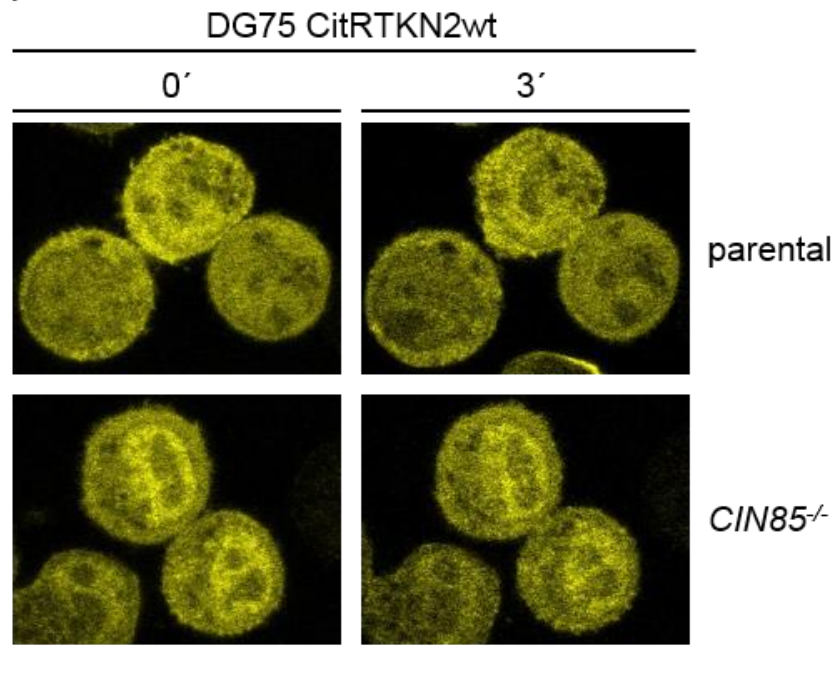

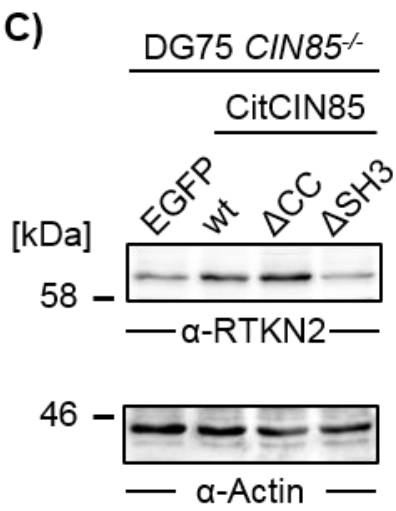

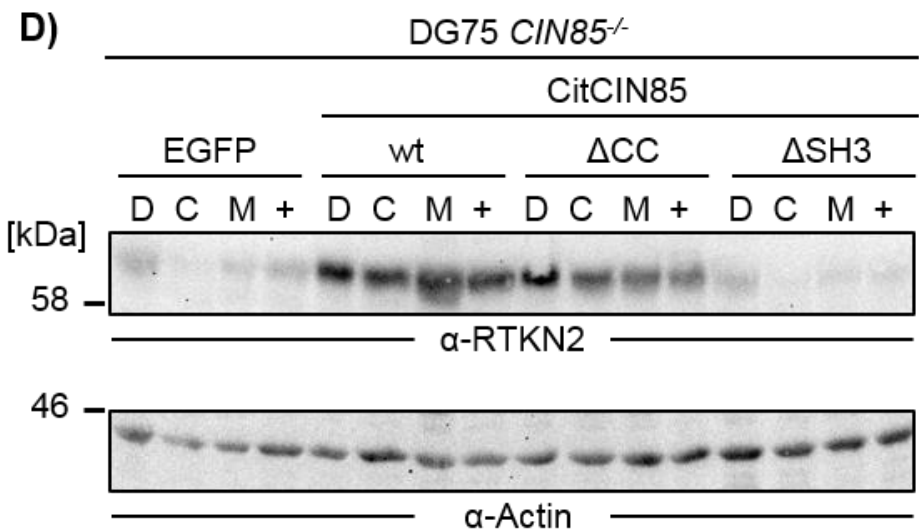

Figure 3.29: RTKN2 is ubiquitously localized and its stability is mediated by CIN85. A) Parental and CIN85-deficient DG75 cells were retrovirally transduced with constructs coding for citrine-tagged wild-type RTKN2 and the expression levels were analyzed by flow cytometry. B) The cells described in A) were subjected to laser scanning microscopy and left untreated (left panel) or stimulated with $10 \mu \mathrm{g} / \mathrm{ml}$ anti-lgM $\mathrm{F}\left(\mathrm{ab}^{\prime}\right)_{2}$ fragments for $3 \mathrm{~min}\left(3^{\prime}\right)$ (right panel). C) Cleared cellular lysates of CIN85-deficient DG75 cells expressing EGFP, CitCIN85wt (wt), CitCIN85 $\Delta \mathrm{CC}(\Delta \mathrm{CC})$ or CitCIN85 $\Delta \mathrm{SH}_{3}\left(\Delta \mathrm{SH}_{3}\right)$ were subjected to SDSPAGE and the immunoblot was probed with antibodies detecting RTKN2 and Actin. D) CIN85-deficient DG75 
cells expressing EGFP or CitCIN85wt were either treated with DMSO (D), $25 \mu \mathrm{g} / \mathrm{ml} \mathrm{CHX} \mathrm{(C),} 20 \mu \mathrm{M}$ MG132 (M) or a combination of both (+) for 10 hours. Immunoblot of cleared cellular lysates was stained with antiRTKN2 and anti-Actin antibodies as controls. The respective molecular weight of marker proteins (in $\mathrm{kDa}$ ) is indicated on the left.

To analyze this in more detail, the translation inhibitor $\mathrm{CHX}$ and the proteasome inhibitor MG132 were applied to CIN85-deficient DG75 cells expressing either EGFP or CIN85wt for $10 \mathrm{~h}$. DMSO represented the untreated control (figure 3.29.D). CHX alone could not abolish RTKN2 protein levels completely. This indicates a lower turnover rate of RTKN2 compared to SLP65, which was almost completely degraded after $10 \mathrm{~h}$. MG132 treatment accumulated RTKN2 protein to a small extend. Combined treatment of CHX and MG132 kept protein expression levels of RTKN2 comparable to the DMSO treated control. This indicates a role of CIN85 SH3 domains in stabilizing not just proteins with high but also low turnover rates by direct interaction. 


\section{DISCUSSION}

Activation of $\mathrm{B}$ cells via the $\mathrm{BCR}$ leads to the formation of the $\mathrm{Ca}^{2+}$ initiation complex and its subsequent recruitment to the plasma membrane, followed by the generation of lipid derived second messengers leading to enrichment of cytosolic $\mathrm{Ca}^{2+}$. The increased levels of second messengers result in the regulation of transcription factor activation. This is not just important for B cell development but also for B cell activation and further differentiation. However, there are still gaps of knowledge in BCR signal transduction. One important factor in this pathway is the adaptor protein CIN85, which constitutively interacts with and thereby promotes the function of SLP65, the key adaptor protein of the $\mathrm{Ca}^{2+}$ initiation complex. In addition, also the BCR distant activation process of the transcription factor NF-KB is supported by CIN85. CIN85 deficiency in humans is presented with defective $B$ cell activation that exemplifies its importance. Nevertheless, the underlying mechanisms by which CIN85 acts in BCR-induced signaling are not fully understood. Therefore, I investigated CIN85 in my PhD project.

The main achievements of my PhD project are listed below:

- Generation of a CIN85-deficient human DG75 B cell sub-line using the TALEN gene targeting method to study BCR-induced signaling processes.

- BCR-induced SLP65 membrane recruitment and $\mathrm{Ca}^{2+}$ mobilization are promoted by CIN85 in the human DG75 B cell line.

- CIN85 interacts with the cytoplasmic tail of the mlgM, which is enhanced upon stimulation dependent BCR ubiquitinylation.

- CIN85 mediates protein stability of SLP65 by direct binding with its SH3 domains.

- The involvement of CIN85 in BCR-induced NF-KB activation is most likely due to the mediation of PKC $\beta$ plasma membrane localization.

\subsection{CIN85 fine tunes BCR-induced $\mathrm{Ca}^{2+}$ mobilization}

In the beginning of my project, I generated a human DG75 B cell sub-line deficient for CIN85 using the TALEN gene targeting method to have a sophisticated model system for CIN85 investigations regarding genetic and biochemical approaches. In addition, human cell lines enable better comparison with the situation in the CIN85-deficient patient than mouse models and the chicken B cell line DT40, which are both evolutionary more distant. The characterization of the CIN85-deficient DG75 sub-line revealed an important role of CIN85 in BCR-induced $\mathrm{Ca}^{2+}$ mobilization using physiological stimulation conditions. This is in accordance with findings in the CIN85-deficient patient, which underlines the suitability

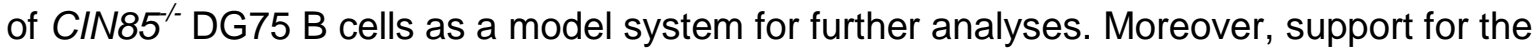


impact of CIN85 in BCR-induced $\mathrm{Ca}^{2+}$ mobilization was obtained by shRNA-mediated knock down of CIN85 in the chicken B cell line DT40 (Bremes, 2012). However, it should be noted that knock down mediated decrease in protein expression has the disadvantage of remaining residual protein amounts.

SLP65, the steady ligand of CIN85, constitutes an essential platform in $\mathrm{Ca}^{2+}$ signaling (Chiu et al., 2002) and hence the meaning of this constitutive interaction was of interest for my project. By reconstitution of $S L P 65^{-}$DG75 cells with a SLP65 variant that is incapable of binding to CIN85 and its homolog CD2AP (SLP65M23), I observed a decreased $\mathrm{Ca}^{2+}$ flux similar to that in CIN85 ${ }^{-\sim}$ DG75 cells upon BCR activation. In contrast, CD2AP deficiency in DT40 cells (Bremes, 2012) or shRNA-mediated decreased CD2AP expression in DG75 cells do not alter $\mathrm{Ca}^{2+}$ mobilization indicating that CIN85 is more important in that context. Nevertheless, CD2AP can partially compensate for the loss of CIN85 in BCR-induced Ca ${ }^{2+}$ flux in DT40 and DG75 cells (Bremes, 2012) (own observed data). These partially overlapping function of CIN85 and CD2AP is likely, since the two proteins developed by gene duplication in early vertebrate evolution (Tossidou et al., 2012) and share high (54 \%) amino acid sequence similarity.

I could confirm the enhancing impact of CIN85 further using the CIN85 ${ }^{\wedge}$ SLP65 DG75 double knock out (DKO) sub-line. Reconstitution of these cells with SLP65 showed a reduced $\mathrm{BCR}$-induced $\mathrm{Ca}^{2+}$ mobilization compared to parental cells, even though ectopic SLP65 expression was enhanced than in parental DG75 cells. Therefore, CIN85 is a prerequisite for complete SLP65 functionality in $\mathrm{Ca}^{2+}$ signaling, which is in line with previous findings of our group obtained in the chicken B cell line DT40. In DT40 cells the constitutive interaction is important for efficient BCR-induced SLP65 plasma membrane recruitment, phosphorylation and hence $\mathrm{Ca}^{2+}$ mobilization (Oellerich et al., 2011). The basis for complete SLP65 function is the oligomerization of CIN85 and hence accumulation of SLP65 (Kühn, 2015). This implicates that complex formation by CIN85 simplifies signal onset and constitutes a fine-tuning mechanism in the context of physiological BCR activation

In contrast to the human DG75 and the chicken DT40 B cells, $\mathrm{Ca}^{2+}$ mobilization in CIN85deficient primary murine B cells was not altered upon BCR stimulation (Kometani et al., 2011). However, it has to be considered that in murine B cells the homolog of SLP65, the adaptor protein SLP76, is expressed (Mizuno et al., 2005), which is missing in chicken and human B cells and is only found in T cells instead. SLP76 does not interact with CIN85 and hence does not depend on CIN85 support. Therefore, SLP76 could replace SLP65 in murine CIN85-deficient B cells. Nevertheless, Kometani and colleagues reported that the $B$ cell specific CIN85 deficiency resulted in a reduction of the B1 B cell 
compartment and a diminished $\mathrm{T}$ cell-independent ( $\mathrm{TI}$ ) type II immune response (Kometani et al., 2011). For differentiation into B1 B cells as well as initiation of a TI type II immune response, stimulation of the BCR with antigens that contain repeating units and mediate crosslinking of neighboring BCRs is essential (Hayakawa et al., 1999; Vos et al., 2000). In contrast to these findings in primary murine B cells, the group of Akashi constitutes CIN85 as an inhibitory protein in BCR-mediated signaling in the human B cell line BJAB (Niiro et al., 2012). The authors claim that ectopic CIN85 expression has a diminishing function in BCR-induced phosphorylation of the signaling proteins Syk, SLP65 and PLCy2 leading to reduced $\mathrm{Ca}^{2+}$ mobilization whereas shRNA-mediated knock down of CIN85 resulted in the contrary.

Experiments with ectopically expressed CIN85 in the human B cell line Ramos are not in accordance with the reported inhibitory function of CIN85 in BCR-induced $\mathrm{Ca}^{2+}$ mobilization (data not shown). Niiro et al. additionally reported that CIN85 knock down in human primary B cells initiated BCR-induced survival, growth and differentiation, which is in contrast to the normal B cell numbers and compartments in the CIN85-deficient patient (unpublished data). An explanation for this contradictory results might be the usage of different cell lines and/or experimental systems. Noteworthy, my findings in human DG75 cells are underpinned by reconstitution experiments and reflect the situation in the patient.

Requirement as well as consequence of $\mathrm{BCR}$-induced $\mathrm{Ca}^{2+}$ mobilization is the tyrosine phosphorylation of several signal transducing factors. In CIN85-deficient DG75 cells, overall tyrosine phosphorylation kinetics of proteins are unaltered. Hence, the observed interactions between CIN85 and the BCR proximal tyrosine kinases Lyn and Syk are not crucial for the function of these kinases. In addition, other tyrosine kinases were not identified as binding partners of CIN85 so far.

With regard to further downstream phosphorylation events, it is published that the balance between CIN85 and CD2AP expression determines Akt/Erk signaling in different developmental stages of podocytes (Tossidou et al., 2007). Moreover, CIN85 interaction with the p85a subunit of PI3K is reported to inhibit the lipid kinase activity of PI3K and thereby downstream Akt signaling in in vitro experiments (Gout et al., 2000). In contrast, loss of CIN85 in the human B cell line DG75 did not alter Akt and Erk phosphorylation upon BCR activation, which is in agreement with results obtained in CIN85-deficient primary murine (Kometani et al., 2011) as well as primary human B cells of the patient (unpublished data).

Collectively, the available data show discrepancies regarding the function of CIN85 in BCR-induced signaling in different species, cell types and developmental stages. Noteworthy, the generated human CIN85-deficient DG75 B cell sub-line resembled so far 
the phenotype of the human patients showing the importance of CIN85 in BCR-induced $\mathrm{Ca}^{2+}$ mobilization. In that context, my results confirm the previous findings of our group obtained in chicken DT40 cells, which constitute CIN85 as the critical binding partner of SLP65, also in the human B cell line DG75 (Oellerich et al., 2011). Via this interaction, CIN85 mediates its enhancing function in $\mathrm{Ca}^{2+}$ response upon BCR stimulation, but does not influence overall tyrosine phosphorylation.

\subsection{CIN85 acts in BCR proximal events}

Requisite for BCR-initiated protein phosphorylation and $\mathrm{Ca}^{2+}$ flux is the translocation of effector proteins to the plasma membrane and in the close proximity of the BCR. CIN85 constitutes an enhancing role in $\mathrm{BCR}$-induced $\mathrm{Ca}^{2+}$ response and is recruited to the plasma membrane upon BCR stimulation. For both processes the three $\mathrm{SH} 3$ as well as the coiled coil (CC) domains are indispensable. However, the exact mechanism by which CIN85 is recruited to the plasma membrane remains unknown. Zhang and colleagues reported that CIN85 interacts with phosphatidic acid (PA) and that the CIN85 CC domain mediates this interaction (Zhang et al., 2009). In contrast, Julius Kühn provided evidence that PA association is not crucial for plasma membrane translocation of CIN85 (Kühn, 2015). If CIN85 does not interact with membranes directly, this association could be mediated by an interaction partner that is present in the plasma membrane. But so far none of the known CIN85 binding partners has been shown to fulfill this requirement. Also Lyn, which is resident in the plasma membrane and interacts with CIN85, is not involved in CIN85 membrane recruitment. Nevertheless, the number of potential recruitment partners can be narrowed down to substrates of the BCR-proximal kinase Lyn, since expression of a kinase-inactive Lyn variant in Lyn-deficient DT40 B cells showed a defect of CIN85 plasma membrane translocation (Bremes, 2012).

4.2.1 The kinetic of SLP65 plasma membrane translocation depends on CIN85 in human B cells

Although the mechanism of CIN85 plasma membrane is not completely understood, its importance for BCR-induced SLP65 plasma membrane translocation is established in DT40 B cells. In these cells, SLP65 recruitment depends on the interaction with CIN85 (Oellerich et al., 2011). In DG75 B cells, the SLP65 M23 variant, which can neither interact with CIN85 nor CD2AP, requires higher stimulating antibody amounts compared to the recruitment of wild-type SLP65. In addition, translocation of wild-type SLP65 to the 
plasma membrane in CIN85-deficient DG75 B cells occurs delayed compared to CIN85expressing DG75 cells. Collectively, the obtained data show an impact of CIN85 on SLP65 plasma membrane recruitment in the human B cell line DG75, which is however rather supportive than pivotal as in DT40 B cells. In addition, I could show that SLP65 vice versa influences CIN85 plasma membrane recruitment, since the expression of SLP65 slows CIN85 translocation down, which positions the process of CIN85 recruitment upstream of that of SLP65.

Work of our group showed that in resting DT40 B cells SLP65 is accumulated in dot like structures via its $\mathrm{N}$-terminus ( first 50 amino acids), which mediates direct protein-lipid interaction. These dots were characterized as macro-molecular assemblies of SLP65 at intracellular, vesicular compartments (Engelke et al., 2014). The preassembly of SLP65 in resting cells is necessary for its plasma membrane translocation as a SLP65 variant lacking the $\mathrm{N}$-terminus is equally distributed in the cytosol and not recruited to the plasma membrane upon BCR stimulation. Further specification of vesicular targeting of SLP65 is reached by interaction with CIN85 as the SLP65 M23 variant is trapped in the Golginetwork in DT40 cells (Engelke et al., 2014). Although dot like distribution of SLP65 in DG75 cells was only minor in contrast to DT40 cells, Sona Pirkuliyeva could identify these dots as vesicles also in the human B cell line (Pirkuliyeva, 2015). The differences between DG75 and DT40 B cells lead to the hypothesis that SLP65 might use different recruitment mechanisms. One possible explanation might be the difference regarding the developmental stage, since DT40 cells are immature, whereas DG75 cells represent a mature B cell line. Also the origin of different species can be of impact. Both development and origin can result in the expression of different interactors of SLP65 that participate in its translocation.

\subsubsection{CIN85 is directly recruited into BCR signalosomes}

As shown by TIRF microscopy, both CIN85 and SLP65 are not only recruited to the plasma membrane but also colocalize with BCR containing microclusters. Therefore, it is possible that CIN85 supports the localization of SLP65 to the BCR where SLP65 can interact via its $\mathrm{SH} 2$ domain with a non-ITAM motif in the cytoplasmic tail of Iga and is subsequently phosphorylated by Syk (Engels et al., 2001). In my PhD project, I demonstrated in affinity purification experiments that CIN85 associates with the BCR. However, neither the BCR cytoplasmic tail nor the BCR associated Ig $\alpha / \lg \beta$ cytoplasmic tails contain putative CIN85 interaction sites, so that the mechanism of BCR association is not clear. 
Since I observed that CIN85 can bind to Lyn, their interaction might be a critical factor for CIN85-BCR association. Lyn is resident in membranes and phosphorylates the ITAMs in the $\lg \alpha / \lg \beta$ heterodimer as a first step after antigen binding (Saouaf et al., 1994). Therefore, binding of CIN85 to Lyn would locate CIN85 in close proximity to the BCR. However, the exact mechanism and importance of this interaction needs to be unraveled in future experiments. In B cells, Lyn also acts downstream of the co-receptors CD19, CD40 and the regulatory receptor CD22 (Ren et al., 1994; Cornall et al., 1998; Fujimoto et al., 1998). Therefore, it is possible that CIN85 is involved in these signaling cascades, too, which is not reported yet.

\subsubsection{Ubiquitinylation events calibrate CIN85's BCR-proximal interaction network} In the literature, interaction of CIN85 with other receptors than the BCR is reported in different cell types (Petrelli et al., 2002; Hutchings et al., 2003). For example, the inducible interaction of CIN85 with cbl is thought to mediate the association between CIN85 and the EGFR (Soubeyran et al., 2002; Kobayashi et al., 2004). In this trimolecular complex the EGFR is ubiquitinylated and hence internalized and degraded. In the last years, other publications claimed that CIN85 is not involved in endocytosis but degradation of the EGFR. Nevertheless, association of CIN85 with receptors involves their cytoplasmic ubiquitinylation by the inducible interactor of CIN85, namely cbl.

I could provide evidence that also the BCR heavy chain cytoplasmic tail is ubiquitinylated upon BCR stimulation in the human Ramos B cell line. This is in line with reports by Drake and colleagues who analyzed BCR ubiquitinylation in mice (Drake et al., 2006). The IgM heavy chain cytoplasmic tail consists of only the three amino acids lysine-valine-lysine (KVK) with lysines being the site of ubiquitinylation. I showed that the cytoplasmic KVK tail is ubiquitinylated upon BCR stimulation, which leads to the assumption that this takes place in the other KVK containing immunoglobuline subtypes ( $\lg D, \lg G$ and $\lg E$ ), too. Change of lysines to arginines resulted in the loss of stimulation dependent ubiquitinylation. As NMR analysis (Bezsonova et al., 2008) and my biochemical ubiquitinagarose affinity purification experiments revealed interaction of CIN85 with ubiquitin, I examined whether the ubiquitinylated KVK tail is a possible binding site for CIN85. My results revealed that ubiquitinylation of the BCR cytoplasmic tail is involved in CIN85-BCR association. Both ubiquitinylation of the BCR and CIN85-BCR association depend on BCR stimulation. Ubiquitin mediated interactions are commonly weak $\left(K_{d}>100 \mu M\right)$ (Hurley et al., 2006) and could support interactions mediated by other motifs in the ubiquitinylated protein and thereby enhance specificity (Hicke et al., 2005). Next to the ubiquitinylated cytoplasmic tail of the BCR as interactor of CIN85 also Syk, which is ubiquitinylated upon 
BCR stimulation (Rao et al., 2001; Sohn et al., 2003) interacts with CIN85. However, this interaction appears to be weak, which might be due to binding of the CIN85 SH3 domains to the ubiquitin modification of Syk and not Syk directly. The observed weak interactions of CIN85 with Syk, Lyn and the BCR could act in concert with the interaction between CIN85 and cbl to mediate CIN85 localization at the site of the BCR and thus provide multiple steps of regulation.

As CIN85 is not only capable of interacting with ubiquitin but is also mono- or diubiquitinylated by $\mathrm{cbl}$ (Bezsonova et al., 2008), it might interact with other proteins that harbor ubiquitin binding domains. CIN85 intramolecular interaction is not only reported for its $\mathrm{SH} 3$ domain to a proline rich motif but also to a monoubiquitin modification (Bezsonova et al., 2008). Monoubiquitin mediated intramolecular interactions are described to prevent intermolecular ubiquitin binding (Hoeller et al., 2006). In that context, monoubiquitinylation of CIN85 is reported to compete with cbl interaction and therefore cbl-mediated function in EGFR endocytosis in HEK 293 cells (Bezsonova et al., 2008). It is possible that cbl mediated monoubiquitinylation of CIN85 in B cells hinders CIN85-BCR interaction and hence constitutes another regulatory mechanism of CIN85 recruitment.

\subsubsection{The cytoplasmic tail of the mlgM determines BCR complex stability}

The amino acid substitution of KVK to RVR within the cytoplasmic tail of the BCR revealed next to the loss CIN85 interaction also instability of the multimeric BCR complex. Affinity purification of the surface BCR using even mild detergent conditions ( $0.5 \%$ NP40) showed no interaction with the $\lg \alpha / \lg \beta$ heterodimer in resting and stimulated DG75 B cells. So far it is published that BCR binding of moderate- to low-affinity antigens destabilizes the BCR complex (Kouskoff et al., 1998; Vilen et al., 1999). The weakened BCR complex stability based on the RVR substitution did not alter overall tyrosine phosphorylation events and $\mathrm{Ca}^{2+}$ mobilization downstream of the BCR. This supports the common concept claiming that the ITAMs in the cytoplasmic tails of both $\lg \alpha$ and $\lg \beta$ are the main motifs in signaling initiation (Shaw et al., 1990; Sanchez et al., 1993; Luisiri et al., 1996). In contrast, substitution of the amino acid residues tyrosine-serine (YS) to valinevaline (VV) in the C-terminal end of the transmembrane part of the murine IgM weakens the interaction between $\mathrm{mlgM}$ and $\lg \alpha / \lg \beta$, which interferes with $\mathrm{BCR}$ signaling and antigen targeting functions (Stevens et al., 1994; Parent et al., 2002). Because mlg surface expression depends on the association with the $\lg \alpha / \lg \beta$ heterodimer (Venkitaraman et al., 1991; Costa et al., 1992; Matsuuchi et al., 1992), the association between the chimeric $\gamma 2 a R V R$ and the $\lg \alpha / \lg \beta$ heterodimer was not altered, since the chimeric BCR was still expressed on the B cell surface. For BCR complex association, the 
transmembrane domains of the $\lg$ heavy chain as well as the $\lg \alpha / \lg \beta$ heterodimer and the extracellular part of $\lg \beta$ are known to be crucial (Grupp et al., 1995; Condon et al., 2000), which are unchanged in the chimeric Y2aRVR BCR. Nevertheless, I observed weakened interaction of $\mathrm{Y} 2 \mathrm{aRVR}$ with the $\lg \alpha / \lg \beta$ heterodimer in $\mathrm{BCR}$ affinity purifications that is also reported for KVK substitutions to either lysine-arginine $(K R)$ or arginine-isoleucine-arginine (RIR) (Shaw et al., 1990; Blum et al., 1993). Complex assembly and surface expression of the KR and RIR multimeric BCR complexes are also unaffected.

These substitutions experiments are in contrast to truncation experiments of the BCR cytoplasmic tail. KVK truncation was reported to result in decreased IgM surface expression and signaling strength (Blum et al., 1993). This IgM mutant did also not copurify the $\lg \alpha / \lg \beta$ heterodimer and is described to be covalently linked to phosphatidylinositol instead of being expressed as an integral transmembrane protein (Mitchell et al., 1991). Therefore, the interaction with the transmembrane $\lg \alpha / \lg \beta$ heterodimer is lost and hence signaling is abolished. The available data collectively indicate that substitution of the IgM cytoplasmic tail residues by others of the same charge allow receptor surface expression and activation to initiate tyrosine phosphorylation and $\mathrm{Ca}^{2+}$ signaling, whereas truncation of KVK inhibits these events.

In my experiments the $\lg \alpha / \lg \beta$ heterodimer did co-purify with $\gamma 2 a R V R$ neither in resting nor stimulated cells. As ubiquitinylation of the cytoplasmic tail did only occur upon BCR stimulation, I can exclude that this modification is responsible for the diminished BCR stability. Since lysines can be also modified in different manners like methylation (Chuikov et al., 2004) or sumoylation (Matunis et al., 1996; Mahajan et al., 1998) events, these might be involved in BCR complex stability regulation.

\subsubsection{Internalization of antigen-bound BCR does not depend on the BCR's cytoplasmic region}

Since ubiquitinylation of other types of receptors, like the EGFR, induces internalization, I tested this in the context of the BCR. Here, I could not observe any changes in the stimulation dependent internalization rates induced by cytoplasmic tail amino acid substitution, which is in accordance with the work of the Drake group (Drake et al., 2006). BCR internalization is already known to depend on the ITAM motif in $\lg \beta$ (Gazumyan et al., 2006) and obviously the ubiquitinylation of the heavy chain cytoplasmic tail does not contribute. Drake and colleagues could additionally see that inhibition of the proteasome activity alters the intracellular trafficking of internalized antigen-BCR complexes, and selectively blocks the BCR-mediated processing and presentation of cognate antigen (Drake et al., 2006). Trafficking of internalized antigen-BCR complexes occurs within MHC 
class II-containing multivesicular structures, termed MIICs (Neefjes et al., 1990; Kleijmeer et al., 1996), and uses similar mechanisms to the well-established EGFR processing machinery (Katzmann et al., 2001; Dikic, 2003; Hierro et al., 2004). Based on that, Drake and colleagues propose the EGFR processing machinery to represent the paradigm for that of the BCR (Drake et al., 2006). In the case of EGFR endocytosis, cbl mediates ubiquitinylation of the cytoplasmic tail. An impact of $\mathrm{cbl}$ is also reported for BCR internalization (Kitaura et al., 2007) but likely not due to ubiquitinylation. CIN85, as an inducible ligand of $\mathrm{cbl}$, is also described to influence EGFR internalization. Involvement of CIN85 was not observed for BCR endocytosis in human DG75 B cells and this is in concert with experiments performed in DT40 CIN85 ${ }^{\text {sh }}$ (Bremes, 2012) and primary mouse B cells (Kometani et al., 2011). Also for EGFR internalization, CIN85 influence is a matter of debate, whereas its role in endocytotic trafficking is accepted so far. Therefore, it is likely that CIN85 is not involved in BCR endocytosis but BCR trafficking, a process in which ubiquitinylation is of impact (Drake et al., 2006).

\subsection{Identification of a new CIN85 function: protein stabilization}

Besides the regulation of plasma membrane recruitment of proteins like SLP65, I could show that CIN85 also controls protein stability of distinct interaction partners. In that context, I detected decreased SLP65 protein levels in CIN85-deficient DG75 B cells. Reconstitution experiments revealed that only the SH3 domains of CIN85, which bind to the atypical proline-rich motifs in SLP65, are necessary for stabilization of SLP65. This opens the possibility that CIN85 either directly stabilizes SLP65 by binding or by masking an ubiquitinylation site, which would target SLP65 to degradation. Our group showed in cooperation with the Griesinger group that the central part of SLP65 is intrinsically disordered. Proteins that are intrinsically unstructured (IUPs) are known to mostly coordinate regulatory or mediate signaling events (lakoucheva et al., 2002; Ward et al., 2004). The unstructured nature of proteins is reported to be of benefit and provide advantages like a large interaction surface, access to short linear interaction motifs or presence of molecular recognition elements that fold upon binding (Wright and Dyson, 1999; Oldfield et al., 2005; Tompa, 2005). Proteins with unstructured regions have a short half-life, which I could also show for SLP65. It is reported that structural changes due to phosphorylation or interaction can extend the half-life of these proteins ( $\mathrm{Ngoc}$ et al., 2014; Hsieh et al., 2015). However, additional NMR studies of the SLP65 structure upon in vitro phosphorylation by Syk or in complex with the CIN85 SH3 domains did not show structural changes upon interaction (Pirkuliyeva, 2015). Therefore, it can be excluded that CIN85 interaction with SLP65 provides conformational support and thereby enhances its 
half-life. The CIN85 mediated support for protein stability was additionally identified for RTKN2. This interaction is also mediated by the CIN85 SH3 domains and is sufficient to restore RTKN2 protein levels. Nothing is known about the structure of RTKN2, but in contrast to SLP65, RTKN2 has a longer protein half-life, which indicates that RTKN2 is not as intrinsically disordered as SLP65, so that it is unlikely that binding of CIN85 would support the structural formation.

In conclusion, the available data bring another protein stabilization mechanism into the focus, which is based on masking of potential degradation signals by binding to the interaction motifs in SLP65 or RTKN2. In the preformed complex between CIN85 and SLP65 proteolytic signals could be sterically regulated like it is the case for NF-kB binding to IKBa. NF-KB masks the PEST domain of IKBa and thereby protects it from proteasomal degradation and enhances its half-life (Mathes et al., 2008). I observed that SLP65 is degraded by the proteasome pathway, which is in line with described ubiquitinylation sites upon BCR stimulation (K37, 38, 42) (Wang et al., 2012). Analyzation of additional putative ubiquitinylation sites in SLP65 using the ubiquitin site prediction software from the Rockefeller University did anticipate numerous ubiquitinylation sites of which some are in proximity (8-10 amino acid residues) to the CIN85 interaction motifs. As SLP65 is shown to be intrinsically unstructured in this region, it is hard to tell whether CIN85 binding would prevent possible ubiquitinylation of SLP65. Analyses of SLP65 upon proteasome and translation inhibition revealed that besides proteasomal degradation another pathway must be responsible for SLP65 turnover. In that context, it is described that newly synthesized proteins are also degraded via lysosomal proteolysis (Hampton, 2002; Kostova and Wolf, 2003), which needs to be tested in future experiments. Collectively, the available data support the possibility that CIN85 acts as a critical regulator of protein stability for interacting proteins. This new mechanism could be of high importance for signaling processes and needs to be unraveled in the future.

\subsection{BCR-induced NF-KB signaling is regulated by CIN85}

The previously described property of CIN85 to mediate the formation of protein complexes is not solely restricted to support SLP65 in the $\mathrm{Ca}^{2+}$ initiation complex and its own BCR localization, but could be also involved in the signaling pathway of NF-kB. Since primary $B$ cells from a patient, who lacks CIN85 protein expression, show a blockage in NF-KB activation downstream of the BCR (unpublished data), we know that CIN85 constitutes an important protein in this process. Involvement of CIN85 in the NF-kB pathway was also observed in CIN85-deficient mouse B cells (Kometani et al., 2011) and DT40 chicken B cells (Oellerich et al., 2011). The group of Tomohiro Kurosaki showed that CIN85 links the 
BCR to canonical IKK $\beta$ activation in mice. Noteworthy, the BAFF-mediated noncanonical NF-KB pathway was unaffected (Kometani et al., 2011). However, the site and mechanism of CIN85 interaction in NF-kB activation remained unclear.

A possible linkage of CIN85 to the NF-KB pathway is its binding partner RTKN2, which was identified as the only protein of the CIN85 interactome (Kühn, 2015) being involved in the NF-KB pathway. It is reported to upregulate phosphorylation of the NF-KB subunit p65 and to enhance survival when ectopically expressed in HEK 293 cells (Collier et al., 2009). For its paralog Rhotekin (RTKN), an influence on NF-KB activation is also reported (Liu et al., 2004). I could observe a supportive effect of RTKN2 in NF-kB activation in the chicken DT40 B cell line. Decreased RTKN2 protein levels led to a defective $\mathrm{Ca}^{2+}$ mobilization compared to the parental cells. The phenotype in $\mathrm{Ca}^{2+}$ mobilization as well as NF-KB activation resembled that of shRNA-mediated knock down of CIN85 in DT40 B cells. This indicates that interaction between CIN85 and RTKN2 is of importance in BCRinduced activation in that context. In contrast, reduced RTKN2 protein levels in the human DG75 B cell line did neither impair $\mathrm{Ca}^{2+}$ mobilization nor NF-kB activation. This suggests that the role of RTKN2 in B cells is restricted to the organism and/or the developmental stage. In contrast, functions of CIN85 in chicken DT40 and human DG75 B cells are consistent although to variable extents. Since RTKN2 is not involved in NF-kB activation in human B cells, another site of CIN85 interaction must be of importance in that context. Therefore, I focused at first on PKC $\beta$, which initiates the canonical NF-KB pathway upon $\mathrm{BCR}$ stimulation. In $\mathrm{B}$ cells two isoforms of $\mathrm{PKC} \beta$ are expressed that differ only in their $\mathrm{C}$ terminal 70 amino acids. Whether they fulfill different functions in NF-kB signaling downstream of the BCR is not reported yet. In resting cells, PKC $\beta$ is in an inactive conformation in which the $\mathrm{N}$-terminally located pseudosubstrate intracellularly interacts with the kinase domain. In the N-terminally citrine-tagged PKC $\beta I I$ variant, which I used in this project, it is likely that the pseudosubstrate cannot interact with the kinase domain due to steric exclusion keeping the protein in an active state also in resting cells. Expression of this PKCßII variant in CIN85-deficient DG75 cells resulted in a cytoplasmic distribution whereas in the presence of CIN85 PKC $\beta$ II was largely recruited to the plasma membrane. Hence, CIN85 regulates PKC 3 II localization in human DG75 B cells. The active conformation of the citrine-tagged $\mathrm{PKC} \beta \mathrm{II}$ variant might explain its plasma membrane localization in resting DG75 cells when CIN85 is expressed. Interaction of CIN85 and PKC $\beta$ II was detected, albeit this appeared to be weak and could not be prohibited by destroying a putative binding site of the CIN85 SH3 domains in PKCBII. This is an indication for indirect interaction mediated by one or more accessory proteins. In addition, it is likely that the binding site in PKC $\beta$ II that mediates association with CIN85 is not accessible in the inactive conformation. Strongest interaction between CIN85 and the 
active PKC $\beta I$ II variant was visible in resting cells with diminished binding upon BCR stimulation for $2 \mathrm{~min}$. $5 \mathrm{~min}$ after BCR stimulation, interaction was again enhanced indicating that the interaction is stimulation dependent. Comparison of known CIN85 as well as PKC $\beta$ interactors does not reveal overlapping proteins. Nevertheless, mice deficient of CIN85 or PKC $\beta$ in B cells show similar phenotypes. PKC $\beta$ deficiency in mice led to low $\operatorname{lgM}$ and $\lg \mathrm{G}_{3}$ levels, decreased B1 B cells and reduced TI-II antigen as well as TC dependent responses (Leitges et al., 1996). Also mice that lack B cell specific CIN85 expression show reduced $\operatorname{lgM}$ and $\operatorname{lgG}_{4}$ levels, B1 B cells and $\mathrm{TI}-\mathrm{II}$ antibody responses (Kometani et al., 2011). Hence, regulation of NF-KB by CIN85 is propably connected to the PKC $\beta$ signaling axis. To elucidate this signaling mechanism further, a protein interactome of PKCBII in DG75 human B cells by SILAC-based mass spectrometry is currently under investigations to provide candidate proteins for further biochemical studies.

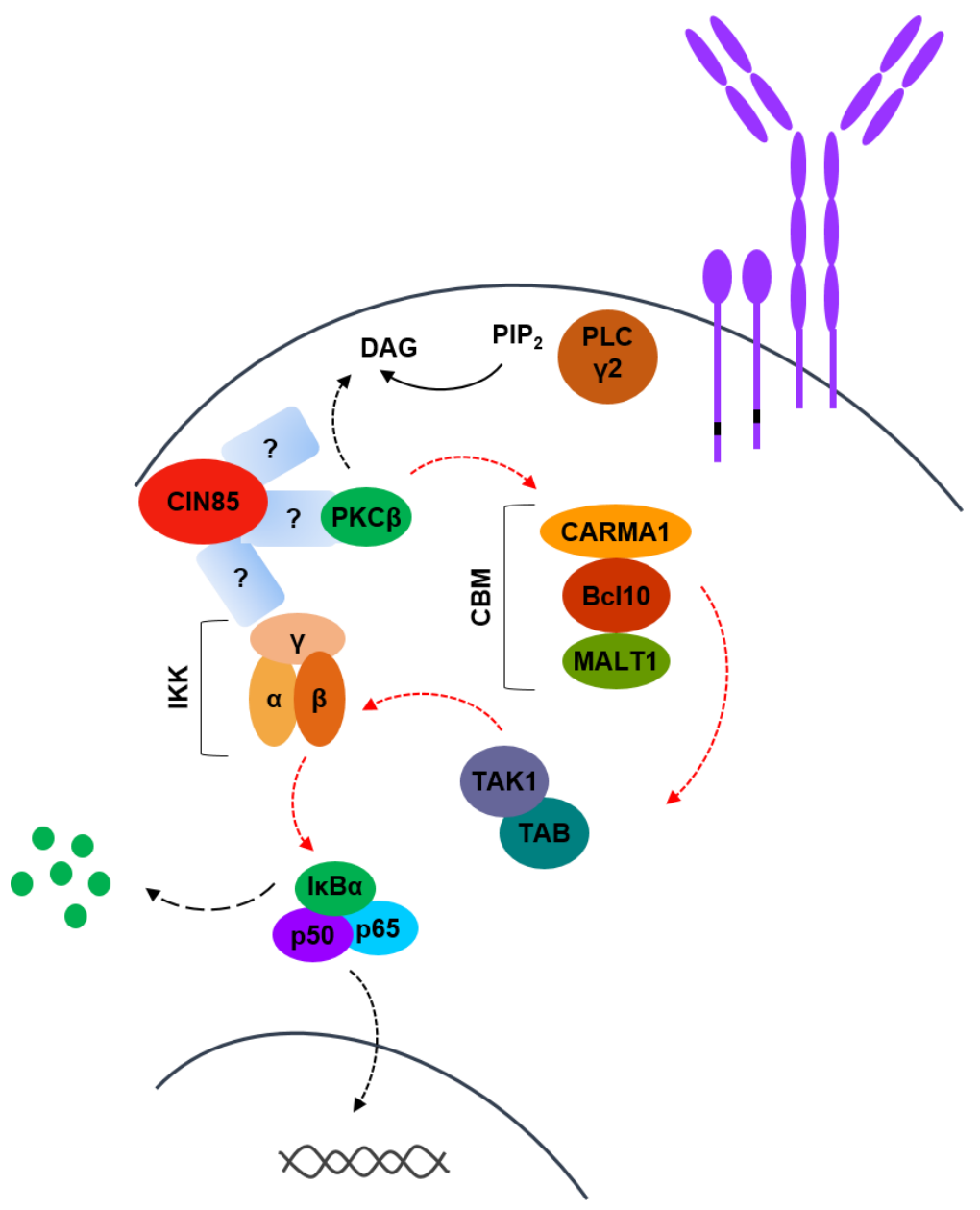

Figure 4.1: Schematic drawing of CIN85 involvement in BCR-induced NF-kB signaling. Upon BCR stimulation, PLCY2 is activated and generates the second messenger DAG. This leads to activation of the canonical NF-KB signaling pathway: $\mathrm{PKC} \beta$ is recruited to the plasma membrane where it is activated by DAG and $\mathrm{Ca}^{2+}$ (not depicted). For membrane translocation of $\mathrm{PKC} \beta$ association with CIN85, which is most likely not 
due to direct binding, is needed. In turn, this leads to phosphorylation of CARMA1 from the CBM complex, followed by TAK1 and subsequent IKK activation. Here, CIN85 might influence IKK complex formation or translocation by indirect interaction with IKKa. Afterwards, phosphorylation of IKBa mediates its degradation and the NF-KB dimer (p50/p65) can translocate into the nucleus to initiate transcriptional processes. Solid black line indicates hydrolysis, dotted black line indicates translocation, dotted red line indicates phosphorylation and dashed black line indicates degradation.

Since I showed that CIN85 influences the plasma membrane localization of PKC $\beta$, where $P K C \beta$ gets in contact with its ligand the second messenger DAG, the localization of the CBM complex directly downstream of PKC $\beta$ might also be influenced by CIN85. The CBM complex consists of CARMA1, Bcl10 and MALT1 (Shinohara et al., 2005). For CARMA1 and Bcl10 no influence of CIN85 expression in terms of their cellular localization was observed. This implicates that CIN85 exclusively mediates PKC $\beta$ plasma membrane localization. However, it has to be considered that Bcl10 and CARMA1 were ectopically expressed in DG75 cells, so that endogenous proteins might hinder their localization processes.

I could additionally show weak and hence probably indirect interaction between CIN85 and IKKa. Also for the IKK complex translocation to the plasma membrane is important (Su et al., 2002). This together with PKC $\beta$ interaction implicates that CIN85 is part of a complex that contains NF-KB signaling proteins and contributes to its formation and cellular localization. 


\section{SUMMARY \& CONCLUSIONS}

From the boy who lacks CIN85 expression resulting in absent serum $\operatorname{lgM}$ and $\operatorname{lgG}_{4}$ antibody levels we know about the importance of CIN85 in BCR-induced signaling. In this patient $\mathrm{Ca}^{2+}$ mobilization and NF-KB activation exclusively downstream of the $\mathrm{BCR}$ are impaired. The work of my PhD project focused on the elucidation of the mechanisms by which CIN85 controls these signaling outcomes in human B cells. In general, the ubiquitiously expressed CIN85 might be present in diverse protein networks fulfilling different adaptor functions. Previous work of our group showed that CIN85 constitutively interacts with the key adaptor protein SLP65 by which CIN85 fine tunes BCR-derived signals in the context of $\mathrm{Ca}^{2+}$ mobilization. With this regard, our group characterized a mechanism by which CIN85 leads to the formation of large multiprotein complexes via oligomerization that constitutes a prerequisite for plasma membrane recruitment. However, the exact process of CIN85 plasma membrane recruitment stayed elusive. My work provides insights into a potential way of CIN85 recruitment. Here, a complex interaction network of direct as well as ubiquitin-mediated bindings is supposed to tether CIN85 in BCR signalosomes in which it thereby might bring its constitutive interaction partners.

Further CIN85 adaptor function is presumably required for NF-kB activation downstream of the BCR. In that context, my work brought a potential signaling axis into the focus by which CIN85 might regulate NF-KB activation, namely CIN85/PKC $\beta$. Here, I showed that CIN85 regulates PKC $\beta$ plasma membrane translocation, which constitutes an essential step in this pathway. Since CIN85 and PKC $\beta$ probably do not directly interact with each other, the complex formation requires involvement of at least one third party, which needs to be elucidated in future experiments.

Besides my findings with regard to complex formation in BCR-induced signaling, I could show for the first time that CIN85 constitutes stability of its constitutive SH3 domain binding partners SLP65 and RTKN2. Hereby, CIN85 might mask potential degradation sites in these proteins that would be otherwise marked by ubiquitinylation for degradation processes. This opens new opportunities for future investigations focusing on a mechanism by which CIN85 might regulate signaling capability of cells.

Collectively, the results of my PhD project show that CIN85 organizes the formation of different protein complexes at different stages of BCR-induced signaling and hence regulates the signaling outcome. Whether the CIN85-mediated complexes are linked to each other or are present in a separate, independent form is so far not known. In combination with CIN85 function as a protein stabilizer, it constitutes a paramount BCRsignaling protein. 


\section{BIBLIOGRAPHY}

Abudula, A., Grabbe, A., Brechmann, M., Polaschegg, C., Herrmann, N., Goldbeck, I., Dittmann, K., and Wienands, J. (2007). SLP-65 signal transduction requires Src homology 2 domain-mediated membrane anchoring and a kinase-independent adaptor function of Syk. The Journal of biological chemistry 282, 29059-29066.

Ahmad, G., Mohapatra, B.C., Schulte, N.A., Nadeau, S.A., Luan, H., Zutshi, N., Tom, E., Ortega-Cava, Cesar, Tu, Chun, Sanada, Masashi, gawa, S., and Toews, M.L., et al. (2014). Cbl-family ubiquitin ligases and their recruitment of CIN85 are largely dispensable for epidermal growth factor receptor endocytosis. The International Journal of Biochemistry \& Cell Biology 57, 123-134.

Baba, T.W., and Humphries, E.H. (1984). Avian Leukosis Virus Infection: Analysis of Viremia and DNA Integration in Susceptible and Resistant Chicken Lines. Journal of virology 51, 123-130.

Ben-Bassat, H., Goldblum, N., Mitrani, S., Goldblum, T., Yoffey, J.M., Cohen, Maimon, M, Bentwich, Z., Ramont, B., Klein, E., and Klein, G. (1977). Establishment in continuous culture of a new type of lymphocyte from a "burkitt-like" malignant lymphoma (line d.g.75). Int. J. Cancer 19, 27-33.

Bezsonova, I., Bruce, M.C., Wiesner, S., Lin, H., Rotin, D., and Forman-Kay, J.D. (2008). Interactions between the three CIN85 SH3 domains and ubiquitin: implications for CIN85 ubiquitination. Biochemistry 47, 8937-8949.

Blum, J.H., Stevens, T.L., and DeFranco, A.L. (1993). Role of the $\mu$ Immunoglobulin Heavy Chain Transmembrane and Cytoplasmic Domains in B Cell Antigen Receptor Expression and Signal Transduction. The Journal of biological chemistry 268, 2723627245.

Bonilla, F.A., Fujita, R.M., Pivniouk, V.I., Chan, A.C., and Geha, R.S. (2000). Adapter proteins SLP-76 and BLNK both are expressed by murine macrophages and are linked to signaling via Fcy receptors I and II/III.

Borinstein, S.S., Hyatt, M.A., Sykes, V.W., Straub, R.E., Lipkowitz, S., Boulter, J., and Bogler, O. (2000). SETA is a multifunctional adapter protein with three SH3 domains that binds Grb2, Cbl, and the novel SB1 proteins. Cellular signalling 12, 769-779.

Bremes, V. (2012). CIN85/CD2AP-based protein complexes in B cell antigen receptor signalling. 
Brown, B.K., and Song, W. (2001). The Actin Cytoskeleton is Required for the Trafficking of the B Cell Antigen Receptor to the Late Endosomes. Traffic (Copenhagen, Denmark) 2, 414-427.

Büchse, T., Horras, N., Lenfert, E., Krystal, G., Körbel, S., Schümann, M., Krause, E., Mikkat, S., and Tiedge, M. (2011). CIN85 interacting proteins in B cells-specific role for SHIP-1. Molecular and cellular proteomics 10.

Burton, D.R., and Woof, J.M. (1992). Human Antibody Effector Function. Advances in Immunology 51, 1-84.

Cahalan, M.D. (2009). STIMulating store-operated $\mathrm{Ca}^{2+}$ entry. Nature cell biology 11, 669677.

Campbell, K.S., Hager, E.J., Fridrich, R.J., and Cambier, J.C. (1991). IgM antigen receptor complex contains phosphoprotein products of B29 and mb-1 genes. Proc Natl Acad Sci U S A 88, 3982-3986.

Cantrell, D. (2015). Signaling in lymphocyte activation. Cold Spring Harb Perspect Biol. 7.

Cermak, T., Doyle, E.L., Christian, M., Wang, L., Zhang, Y., Schmidt, C., Baller, J.A., Somia, N.V., Bogdanove, A.J., and Voytas, D.F. (2011). Efficient design and assembly of custom TALEN and other TAL effector-based constructs for DNA targeting. Nucleic acids research 39, e82 1-11.

Chen, Z.J., Hagler, J., Palombella, V.J., Melandri, F., Scherer, D., Ballard, D., and Maniatis, T. (1995). Signal-induced site-specific phosphorylation targets IKBa to the ubiquitin-proteasome pathway. Genes and Development 9, 1586-1597.

Chen, Z.J., Parent, L., and Maniatis, T. (1996). Site-Specific Phosphorylation of IkBa by a Novel Ubiquitination-Dependent Protein Kinase Activity. Cell 84, 853-862.

Cheng, P.C., Dykstra, M.L., Mitchell, R.N., and Pierce, S.K. (1999). A Role for Lipid Rafts in B Cell Antigen Receptor Signaling and Antigen Targeting. Journal of Experimental Medicine 190, 1549-1560.

Chiu, C.W., Dalton, M., Ishiai, M., Kurosaki, T., and Chan, A.C. (2002). BLNK: molecular scaffolding through 'cis'-mediated organization of signaling proteins. EMBO Journal 21, 6461-6472.

Chuikov, S., Kurash, J.K., Wilson, J.R., Xiao, B., Justin, N., Ivanov, G.S., McKinney, K., Tempst, P., Prives, C., and Gamblin, S.J., et al. (2004). Regulation of p53 activity through lysine methylation. Nature 432, 353-360. 
Collier, F.M., Loving, A., Baker, A.J., and McLeod, Janet, Kirkland, Mark A. (2009). RTKN2 Induces NF-KappaB Dependent Resistance to Intrinsic Apoptosis in HEK cells and Regulates BCL-2 Genes in Human CD4+ Lymphocytes. Journal of Cell Death 2, 9-23.

Condon, C., Hourihane, S.L., Dang-Lawson, M., Escribano, J., and Matsuuchi, L. (2000). Aberrant Trafficking of the B Cell Receptor Ig-Subunit in a B Lymphoma Cell Line. The Journal of Immunology 165, 1427-1437.

Cornall, R.J., Cyster, J.G., Hibbs, M.L., Dunn, A.R., Otipoby, K.L., Clark, E.A., and Goodnow, C.C. (1998). Polygenic Autoimmune Traits: Lyn, CD22, and SHP-1 Are Limiting Elements of a Biochemical Pathway Regulating BCR Signaling and Selection. Immunity 8 , 497-508.

Costa, T.E., Franke, R.R., Sanchez, M., Misulovin, Z., and Nussenzweig, M.C. (1992). Functional Reconstitution of an Immunoglobulin Antigen Receptor in T Cells. Journal of Experimental Medicine 175, 1669-1676.

Dai, L., Aye Thu, C., Liu, X.-Y., Xi, J., and Cheung, Peter C F (2012). TAK1, more than just innate immunity. IUBMB life $64,825-834$.

Damen, J.E., Liu, L., Rosten, P., Humphries, R.K., Jefferson, A.B., Majerus, P.W., and Krystal, G. (1995). The $145-\mathrm{kDa}$ protein induced to associate with Shc by multiple cytokines is an inositol tetraphosphate and phosphatidylinositol 3,4,5-trisphosphate 5phosphatase. Biochemistry 93, 1689-1693.

Davies, G.C., Ettenberg, S.A., Coats, A.O., Mussante, M., Ravichandran, S., Collins, J., Nau, M.M., and Lipkowitz, S. (2004). Cbl-b interacts with ubiquitinated proteins; differential functions of the UBA domains of c-Cbl and Cbl-b. Oncogene 23, 7104-7115.

Deng, L., Wang, C., Spencer, E., Yang, L., Braun, A., You, Jianxin, Slaughter, Clive, Pickart, C., and Chen, Z.J. (2000). Activation of the IKB Kinase Complex by TRAF6 Requires a Dimeric Ubiquitin-Conjugating Enzyme Complex and a Unique Polyubiquitin Chain. Cell 103, 351-361.

Dikic, I. (2002). CIN85/CMS family of adaptor molecules. FEBS letters 529, 110-115.

Dikic, I. (2003). Mechanisms controlling EGF receptor endocytosis and degradation. Biochemical Society Transactions 31, 1178-1181.

Drake, L., McGovern-Brindisi, E.M., and Drake, J.R. (2006). BCR ubiquitination controls BCR-mediated antigen processing and presentation. Blood 108, 4086-4093.

Dustin, M.L., Olszowy, M.W., Holdorf, A.D., Li, J., Bromley, S., Desai, N., Widder, P., Rosenberger, F., van der Merwe, P. Anton, and Allen, P.M., et al. (1998). A Novel Adaptor 
Protein Orchestrates Receptor Patterning and Cytoskeletal Polarity in T-Cell Contacts. Cell 94, 667-677.

Dykstra, M.L., Cherukuri, A., and Pierce, S.K. (2001). Floating the Raft Hypothesis for Immune Receptors: Access to Rafts Controls Receptor Signaling and Trafficking. Traffic (Copenhagen, Denmark) 2, 160-166.

Egawa, T., Albrecht, B., Favier, B., Sunshine, M.-J., Mirchandani, K., O'Brien, W., Thome, M., and Littman, D.R. (2003). Requirement for CARMA1 in Antigen Receptor-Induced NFKB Activation and Lymphocyte Proliferation. Current Biology 13, 1252-1258.

Engelke, M., Engels, N., Dittmann, K., Stork, B., and Wienands, J. (2007). Ca ${ }^{2+}$ signaling in antigen receptor-activated B lymphocytes. Immunological reviews 218, 235-246.

Engelke, M., Pirkuliyeva, S., Kühn, J., Wong, L., Boyken, J., Hermann, N., Becker, S., Griesinger, C., and Wienands, J. (2014). Macromolecular assembly of the adaptor SLP-65 at intracellular vesicles in resting B cells. Science Signaling 7, 1-8.

Engels, N., Engelke, M., and Wienands, J. (2008). Conformational Plasticity and Navigation of Signaling Proteins in Antigen-Activated B Lymphocytes. Advances in Immunology 97, 251-281.

Engels, N., Wollscheid, B., and Wienands, J. (2001). Association of SLP-65/BLNK with the $B$ cell antigen receptor through a non-ITAM tyrosine of Ig- $\alpha$. Eur. J. Immunol. 31, 2126-2134.

Falasca, M., Logan, S.K., Lehto, V.P., Baccante, G., Lemmon, M.A., and Schlessinger, J. (1998). Activation of phospholipase Cy by PI 3-kinase-induced PH domain-mediated membrane targeting. EMBO Journal 17, 414-422.

Fiala, G.J., Kaschek, D., Blumenthal, B., Reth, M., Timmer, J., and Schamel, Wolfgang W A (2013). Pre-clustering of the B cell antigen receptor demonstrated by mathematically extended electron microscopy. Frontiers in immunology 4, 427.

Fu, C., Turck, C.W., Kurosaki, T., and Chan, A.C. (1998). BLNK: a Central Linker Protein in B Cell Activation. Immunity 9, 93-103.

Fujimoto, M., Poe, J.C., Inaoki, M., and Tedder, T.F. (1998). CD19 regulates B lymphocyte responses to transmembrane signals. Seminars in Immunology 10, 267-277.

Fütterer, K., Wong, J., Grucza, R.A., Chan, A.C., and Waksman, G. (1998). Structural basis for syk tyrosine kinase ubiquity in signal transduction pathways revealed by the crystal structure of its regulatory $\mathrm{SH} 2$ domains bound to a dually phosphorylated ITAM peptide. Journal of molecular biology 281,523537 . 
Gazumyan, A., Reichlin, A., and Nussenzweig, M.C. (2006). Ig beta tyrosine residues contribute to the control of $B$ cell receptor signaling by regulating receptor internalization. The Journal of experimental medicine 203, 1785-1794.

Goitsuka, R., Fujimura, Y.-i., Mamada, H., Umeda, A., Morimura, T., Uetsuka, K., Doi, K., Tsuji, S., and Kitamura, D. (1998). BASH, A Novel Signaling Molecule Preferentially Expressed in B Cells of the Bursa of Fabricius. The Journal of Immunology 116, 58045808.

Gout, I., Middleton, G., Adu Jimi, Ninkina, Natalia N., B.Drobot, L., Filonenko, V., Matsuka, G., M.Davies, A., and Waterfield, M., et al. (2000). Negative regulation of PI 3-kinase by Ruk, a novel adaptor protein. EMBO Journal 19, 4015-4025.

Grupp, S.A., Mitchell, R.N., Schreibert, K.L., McKean, D.J., Abbas, and Abul K. (1995). Molecular Mechanisms that Control Expression of the B Lymphocyte Antigen Receptor Complex. Journal of Experimental Medicine 181, 161-168.

Haglund, K., Shimokawa, N., Szymkiewicz, I., and Dikic, I. (2002). Cbl-directed monoubiquitination of CIN85 is involved in regulation of ligand-induced degradation of EGF receptors. PNAS 99, 12191-12196.

Haglund, K., Sigismund, S., Polo, S., Szymkiewicz, I., Di Fiore, P.P., and Dikic, I. (2003). Multiple monoubiquitination of RTKs is sufficient for their endocytosis and degradation. Nature cell biology 5, 461-466.

Hampton, R.Y. (2002). ER-associated degradation in protein quality control and cellular regulation. Current Opinion in Cell Biology 14, 476-482.

Han, Y., Weinman, S., Boldogh, I., Walker, R.K., and Brasier, A.R. (1999). Tumor Necrosis Factor- $\alpha$-inducible IkBa Proteolysis Mediated by Cytosolic m-Calpain. The Journal of biological chemistry $274,787-794$.

Hara, H., Wada, T., Bakal, C., Kozieradzki, I., Suzuki, S., Suzuki, N., Nghiem, M., Griffiths, E.K., Krawczyk, C., and Bauer, B., et al. (2003). The MAGUK Family Protein CARD11 Is Essential for Lymphocyte Activation. Immunity 18, 763-775.

Havrylov, S., Ichioka, F., Powell, K., Borthwick, E.B., Baranska, J., Maki, M., and Buchman, V.L. (2008). Adaptor protein Ruk/CIN85 is associated with a subset of COPIcoated membranes of the Golgi complex. Traffic (Copenhagen, Denmark) 9, 798-812.

Havrylov, S., Redowicz, M.J., and Buchman, V.L. (2010). Emerging roles of Ruk/CIN85 in vesicle-mediated transport, adhesion, migration and malignancy. Traffic (Copenhagen, Denmark) 11, 721-731. 
Havrylov, S., Rzhepetskyy, Y., Malinowska, A., Drobot, L., and Redowicz, M.J. (2009). Proteins recruited by SH3 domains of Ruk/CIN85 adaptor identified by LC-MS/MS. Proteome science 21.

Hayakawa, K., Asano, M., Shinton, S.A., Gui, M., Allman, D., Stewart, C.L., Silver, J., and Hardy, R.R. (1999). Positive Selection of Natural Autoreactive B Cells. Science (New York, N.Y.) 285, 113-116.

Herrmann, N. (2009). Kooperation funktioneller Domänen des Adapterproteins SLP-65 für die $\mathrm{Ca}^{2+}$-Antwort in B-Lymphocyten.

Hershko, A., and Ciechanover, A. (1998). The ubiquitin system. Annu Rev Biochem 67, 425-479.

Hershko, A., and Heller, H. (1985). Occurrence of a polyubiquitin structure in ubiquitinprotein conjugates. Biochemical and biophysical research communications 128, 10791086.

Hicke, L., and Dunn, R. (2003). Regulation of membrane protein transport by ubiquitin and ubiquitin-binding proteins. Annual review of cell and developmental biology 19, 141-172.

Hicke, L., Schubert, H.L., and Hill, C.P. (2005). Ubiquitin-binding domains. Nature reviews. Molecular cell biology 6, 610-621.

Hierro, A., Sun, J., Rusnack, A.S., Kim, J., Prag, G., Emr, S.D., and Hurley, J.H. (2004). Structure of the ESCRT-II endosomal trafficking complex. Nature 431, 221-225.

Hitzing, C. (2015). Vav guanine nucleotide exchange factors control B cell antigen receptor-induced $\mathrm{Ca}^{2+}$-signaling.

Hoeller, D., Crosetto, N., Blagoev, B., Raiborg, C., Tikkanen, R., Wagner, S., Kowanetz, K., Breitling, R., Mann, M., and Stenmark, H., et al. (2006). Regulation of ubiquitin-binding proteins by monoubiquitination. Nature cell biology $8,163-169$.

Home, W.C., Sanjay, A., Bruzzanti, A., and Baron, R. (2005). The role(s) of Src kinase and $\mathrm{Cbl}$ proteins in the regulation of osteoclast differentiation and function. Immunological reviews 208, 106-125.

Hsieh, L.-S., Su, W.-M., Han, G.-S., and Carman, G.M. (2015). Phosphorylation regulates the ubiquitin-independent degradation of yeast Pah1 phosphatidate phosphatase by the 20 S proteasome. The Journal of biological chemistry 290, 11467-11478.

Humphrey, M.B., Lanier, L.L., and Nakamura, M.C. (2005). Role of ITAM-containing adapter proteins and their receptors in the immune system and bone. Immunological reviews 208, 50-65. 
Hurley, J.H., Lee, S., and Prag, G. (2006). Ubiquitin-binding domains. The Biochemical journal 399, 361-372.

Hutchings, N.J., Clarkson, N., Chalkley, R., Barclay, A.N., and Brown, M.H. (2003). Linking the $\mathrm{T}$ cell surface protein CD2 to the actin-capping protein CAPZ via CMS and CIN85. The Journal of biological chemistry 278, 22396-22403.

lakoucheva, L.M., Brown, C.J., Lawson, J., Obradović, Z., and Dunker, A. (2002). Intrinsic Disorder in Cell-signaling and Cancer-associated Proteins. Journal of molecular biology 323, 573-584.

Ishiai, M., Kurosaki, M., Pappu, R., Okawa, K., Ronko, I., Fu, C., Shibata, M., Iwamatsu, A., Chan, A.C., and Kurosaki, T. (1999). BLNK Required for Coupling Syk to PLCY2 and Rac1-JNK in B Cells. Immunity 10, 117-125.

Iwasaki, A., and Medzhitov, R. (2015). Control of adaptive immunity by the innate immune system. Nature immunology $16,343-353$.

Janeway, C.A., and Medzhitov, R. (2002). Innate immune recognition. Annual review of immunology 20, 197-216.

Jiang, A., Craxton, A., Kurosaki, T., and Clark, E.A. (1998). Different Protein Tyrosine Kinases Are Required for B Cell Antigen Receptor-mediated Activation of Extracellular Signal-Regulated kinase, c-Jun NH2-terminal Kinase 1, and p38 Mitogen-activated Protein Kinase. Journal of Experimental Medicine 188, 1297-1306.

Kabak, S., Skaggs, B.J., Gold, M.R., Affolter, M., West, K.L., Foster, M.S., Siemasko, K., Chan, A.C., Aebersold, R., and Clark, M.R. (2002). The Direct Recruitment of BLNK to Immunoglobulin Couples the B-Cell Antigen Receptor to Distal Signaling Pathways. Molecular and Cellular Biology 22, 2524-2535.

Kanayama, A., Seth, R.B., Sun, L., Ea, C.-K., Hong, M., Shaito, A., Chiu, Y.-H., Deng, L., and Chen, Z.J. (2004). TAB2 and TAB3 activate the NF-KB pathway through binding to polyubiquitin chains. Molecular Cell 15, 535-548.

Karin, M. (1999). The Beginning of the End: IKB Kinase (IKK) and NF-kB Activation. The Journal of biological chemistry $274,27339-27342$.

Karin, M., and Ben-Neriah, Y. (2000). Phosphorylation Meets Ubiquitination: The Control of NF-kB Activity. Annual Review Immunology 18, 621-663.

Katzmann, D.J., Babst, M., and Emr, S.D. (2001). Ubiquitin-Dependent Sorting into the Multivesicular Body Pathway Requires the Function of a Conserved Endosomal Protein Sorting Complex, ESCRT-I. Cell 106, 145-155. 
Kim, M., Tezuka, T., Tanaka, K., and Yamamoto, T. (2004). Cbl-c suppresses v-Srcinduced transformation through ubiquitin-dependent protein degradation. Oncogene 23, 1645-1655.

Kirsch, K.H., Georgescu, M.M., Shishido, T., Langdon, W.Y., Birge, R.B., and Hanafusa, $\mathrm{H}$. (2001). The adapter type protein CMS/CD2AP binds to the proto-oncogenic protein C$\mathrm{Cbl}$ through a tyrosine phosphorylation-regulated Src homology 3 domain interaction. The Journal of biological chemistry $276,4957-4963$.

Kirsch, K.H., Georgescu, M.-M., Ishimaru, S., and Hanafusa, H. (1999). CMS: An adapter molecule involved in cytoskeletal rearrangements. Proc. Natl. Acad. Sci. USA 96, 62116216.

Kitaura, Y., Jang, I.-K., Wang, Y., Han, Y.-C., Inazu, T., Cadera, E.J., Schlissel, M., Hardy, R.R., and Gu, H. (2007). Control of the B Cell-Intrinsic Tolerance Program by c-Cbl and Cbl-b. Immunity 26, 567-579.

Kleijmeer, M.J., Raposo, G., and Geuze, H.J. (1996). Characterization of MHC Class II Compartments by Immunoelectron Microscopy. Methods 10, 191-207.

Klein, G., Giovanella, B., Westman, A., Stehlin, J., and Mumford, D. (1975). An EBVgenome-negative cell line established from an American Burkitt lymphoma; receptor characteristics. EBV infectibility and permanent conversion into EBV-positive sublines by in vitro infection. Intervirology 5, 319-334.

Kobayashi, S., Sawano, A., Nojima, Y., Shibuya, M., and Maru, Y. (2004). The c$\mathrm{Cbl/CD2AP}$ complex regulates VEGF-induced endocytosis and degradation of Flt-1 (VEGFR-1). FASEB journal : official publication of the Federation of American Societies for Experimental Biology 18, 929-931.

Kometani, K., Yamada, T., Sasaki, Y., Yokosuka, T., Saito, T., Rajewsky, K., Ishiai, M., Hikida, M., and Kurosaki, T. (2011). CIN85 drives B cell responses by linking BCR signals to the canonical NF-kappaB pathway. The Journal of experimental medicine 208, 14471457.

Koretzky, G.A., Abtahian, F., and Silverman, M.A. (2006). SLP76 and SLP65: complex regulation of signalling in lymphocytes and beyond. Nature reviews. Immunology 6, 67-78. Kostova, Z., and Wolf, D.H. (2003). For whom the bell tolls: protein quality control of the endoplasmic reticulum and the ubiquitin-proteasome connection. EMBO Journal 22, 23092317. 
Kouskoff, V., Famiglietti, S., Lacaud, G., Lang, P., Rider, J.E., Kay, B.K., Cambier, J.C., and Nemazee, D. (1998). Antigens Varying in Affinity for the B Cell Receptor Induce Differential B Lymphocyte Responses. Journal of Experimental Medicine 188, 1453-1464.

Krappmann, D., and Scheidereit, C. (2005). A pervasive role of ubiquitin conjugation in activation and termination of IKB kinase pathways. EMBO reports 6, 321-326.

Kühn, J. (2015). Multimolecular adaptor protein complexes in B cell receptor signaling.

Laemmli, U. (1970). Cleavage of structural proteins during the assembly of the head of bacteriophage T4. Nature 227, 680-685.

Lehtonen, S., Ora, A., Olkkonen, V.M., Geng, L., Zerial, M., Somlo, S., and Lehtonen, E. (2000). In vivo interaction of the adapter protein CD2-associated protein with the type 2 polycystic kidney disease protein, polycystin-2. The Journal of biological chemistry 275 , 32888-32893.

Leitges, M., Schmedt, C., Guinamard, R., Davoust, J., Schaal, S., Stabel, S., and Tarakhovsky, A. (1996). Immunodeficiency in Protein Kinase Cß-Deficient Mice. Science (New York, N.Y.) 273, 788-791.

Liu, C.-A., Wang, M.-J., Chi, C.-W., Wu, C.-W., and Chen, J.-Y. (2004). Rho/Rhotekinmediated NF-kappaB activation confers resistance to apoptosis. Oncogene 23, 87318742.

Luisiri, P., Lee, Y.J., Eisfelder, B.J., and Clark, M.R. (1996). Cooperativity and Segregation of Function within the $\mathrm{lg}-\alpha / \beta$ Heterodimer of the B Cell Antigen Receptor Complex. journal of biological chemistry $271,5158-5163$.

Mahajan, R., Gerace, L., and Melchior, F. (1998). Molecular Characterization of the SUMO-1 Modification of RanGAP1 and Its Role in Nuclear Envelope Association. The Journal of cell biology 140, 259-270.

Marois, L., Vaillancourt, M., Paré, G., Gagné, V., Fernandes, M J G, Rollet-Labelle, E., and Naccache, P.H. (2011). CIN85 modulates the down-regulation of FcyRlla expression and function by c-Cbl in a PKC-dependent manner in human neutrophils. The Journal of biological chemistry 286, 15073-15084.

Marshall, A.J., Niiro, H., Yun, T.J., and Clark, E.A. (2000). Regulation of B-cell activation and differentiation by the phosphatidylinositol 3-kinase and phospholipase $\mathrm{C}_{Y}$ pathways. Immunological reviews 176, 30-46.

Mathes, E., O'Dea, E.L., Hoffmann, A., and Ghosh, G. (2008). NF-kB dictates the degradation pathway of IKBa. EMBO Journal 27, 1357-1367. 
Matsuuchi, L., Gold, M.R., Travis, A., Grosscheld, R., DeFranco, A.L., and Kelly, R.B. (1992). The membrane IgM-associated proteins MB-1 and Ig- $\beta$ are sufficient to promote surface expression of a partially functional B-cell antigen receptor in a nonlymphoid cell line. PNAS 89, 3404-3408.

Matunis, M.J., Coutavas, E., and Blobel, G. (1996). A Novel Ubiquitin-like Modification Modulates the Partitioning of the Ran-GTPase-activating Protein RanGAP1 between the Cytosol and the Nuclear Pore Complex. The Journal of cell biology 135, 1457-1470.

Minegishi, Y., Rohre, J., Coustan-Smith, E., Lederman, H.M., Pappu, R., Campana, D., Chan, A.C., and Conley, M.E. (1999). An Essential Role for BLNK in Human B Cell Development. Science (New York, N.Y.) 286, 1954-1957.

Mitchell, R.N., Shaw, A.C., Weaver, Y.K., Leder, P., and Abbas, A.K. (1991). Cytoplasmic Tail Deletion Converts Membrane Immunoglobulin to a Phosphatidylinositol-linked Form Lacking Signaling and Efficient Antigen Internalization Functions. The Journal of biological chemistry $266,8856-8860$.

Mizuno, K., Tagawa, Y., Watanabe, N., Ogimoto, M., and Yakura, H. (2005). SLP-76 is recruited to CD22 and dephosphorylated by SHP-1, thereby regulating B cell receptorinduced c-Jun N-terminal kinase activation. Eur. J. Immunol. 35, 644-654.

Morita, S., Kojima, T., and Kitamura, T. (2000). Plat-E: an efficient and stable system for transient packaging of retroviruses. Gene Therapy 12, 1063-1066.

Mullis, K., Faloona, F., Scharf, S., Saiki, R., Horn, G., and Erlich, H. (1986). Specific Enzymatic Amplification of DNA In Vitro: The Polymerase Chain Reaction. Cold Spring Harbor Symposia on Quantitative Biology 51, 263-273.

Muramatsu, M., Kinoshita, K., Fagarasan, S., Yamada, S., Shinkai, Y., and Honjo, T. (2000). Class Switch Recombination and Hypermutation Require Activation-Induced Cytidine Deaminase (AID), a Potential RNA Editing Enzyme. Cell 102, 553-563.

Myouzen, K., Kochi, Y., Okada, Y., Terao, C., Suzuki, A., Ikari, K., Tsunoda, T., Takahashi, A., Kubo, M., and Taniguchi, A., et al. (2012). Functional variants in NFKBIE and RTKN2 involved in activation of the NF-KB pathway are associated with rheumatoid arthritis in Japanese. PLoS genetics 8, 1-13.

Narita, T., Amano, F., Yoshizaki, K., Nishimoto, N., Nishimura, T., Tajima, T., Namiki, H., and Taniyama, T. (2001). Assignment of SH3KBP1 to human chromosome band $X p 22.1 \rightarrow$ p21.3 by in situ hybridization. Cytogenet Cell Genet 93, 133-134. 
Narita, T., Nishimura, T., Yoshizaki, K., and Taniyama, T. (2005). CIN85 associates with TNF receptor 1 via Src and modulates TNF- $\alpha$-induced apoptosis. Experimental Cell Research 304, 256-264.

Navarro, M.N., Nusspaumer, G., Fuentes, P., Gonzalez-Garcia, S., Alcain, J., and Toribo, M.L. (2007). Identification of CMS as a cytosolic adaptor of the human pTa chain involved in pre-TCR function. Blood 110, 4331-4340.

Neefjes, J.J., Stollorz, V., Peters, P.J., Gueze, H.J., and Ploegh, H.L. (1990). The biosynthetic pathway of MHC class II but not class I molecules intersects the endocytic route. Cell 61, 171-183.

Ngoc, L.V., Wauquier, C., Soin, R., Bousbata, S., Twyffels, L., Kruys, V., and Gueydan, C. (2014). Rapid proteasomal degradation of posttranscriptional regulators of the TIS11/tristetraprolin family is induced by an intrinsically unstructured region independently of ubiquitination. Molecular and Cellular Biology 34, 4315-4328.

Niiro, H., Jabbarzadeh-Tabrizi, S., Kikushige, Y., Shima, T., Noda, K., Ota, S.-I., Tsuzuki, H., Inoue, Y., Arinobu, Y., and Iwasaki, H., et al. (2012). CIN85 is required for Cblmediated regulation of antigen receptor signaling in human B cells. Blood 119, 2263-2273. Nishizuka, Y. (1992). Intracellular Signaling by Hydrolysis of Phospholipids and Activation of Protein Kinase C. Science (New York, N.Y.) 258, 607-614.

Oeckinghaus, A., Hayden, M.S., and Ghosh, S. (2011). Crosstalk in NF-kB signaling pathways. Nature immunology 12, 695-708.

Oellerich, T., Bremes, V., Neumann, K., Bohnenberger, H., Dittmann, K., Hsiao, H.-H., Engelke, M., Schnyder, T., Batista, F.D., and Urlaub, H., et al. (2011). The B-cell antigen receptor signals through a preformed transducer module of SLP65 and CIN85. The EMBO journal 30, 3620-3634.

Oldfield, C.J., Cheng, Y., Cortese, M.S., Romero, P., Uversky, V.N., and Dunker, A.K. (2005). Coupled folding and binding with alpha-helix-forming molecular recognition elements. Biochemistry 44, 12454-12470.

Ota, Y., and Samelson, L.E. (1997). The Product of the Proto-Oncogene c-cbl: A Negative Regulator of the Syk Tyrosine Kinase. Science (New York, N.Y.) 276, 418-420.

Parent, B.A., Wang, X., and Song, W. (2002). Stability of the B cell antigen receptor modulates its signaling and antigen-targeting functions. Eur. J. Immunol. 32, 1839-1846.

Petrelli, A., Gilestro, G.F., Lanzardo, S., Comoglio, P.M., Migone, N., and Giordano, S. (2002). The endophilin-CIN85-Cbl complex mediates ligand-dependent downregulation of c-Met. Nature 416, 187-190. 
Pirkuliyeva, S. (2015). Structural and functional elucidation of the primary transducer module of the $B$ cell antigen receptor.

Putney Jr., J. (1986). A model for receptor-regulated calcium entry. Cell Calcium 7, 1-12.

Rao, N., Ghosh, A.K., Ota, S., Zhou, P., Reddi, A.L., Hakezi, K., Druker, B.K., Wu, J., and Band, H. (2001). The non receptor tyrosine kinase Syk is a target of Cbl-mediated ubiquitylation upon B-cell receptor stimulation. EMBO Journal 20, 7085-7095.

Rawlings, D.J., Sommer, K., and Moreno-García, M.E. (2006). The CARMA1 signalosome links the signalling machinery of adaptive and innate immunity in lymphocytes. Nature reviews. Immunology 6, 799-812.

Ren, C.L., Morio, T., Fu, S.M., and Geha, R.S. (1994). Signal Transduction via CD40 Involves Activation of lyn Ki-ase and Phosphatidylinositol-3-klnase, and Phosphorylation of Phospholipase Cy2. Journal of Experimental Medicine 179, 673-680.

Reth, M. (1989). Antigen receptor tail clue. Nature 338, 383-384.

Reth, M. (1992). Antigen Receptors on B Lymphocytes. Annual Review Immunology 10, 97-121.

Reth, M. (2001). Oligomeric antigen receptors: a new view on signaling for the selection of lymphocytes. Trends in immunology 22, 356-360.

Robertson, H., Hime, G.R., Lada, H., and Bowtell, D.D.L. (2000). A Drosophila analogue of v-Cbl is a dominant-negative oncoprotein in vivo. Oncogene 19, 3299-3308.

Rolli, V., Gallwitz, M., Wossning, T., Flemming, A., Schamel, W.W., Zürn, C., and Reth, M. (2002). Amplification of B Cell Antigen Receptor Signaling by a Syk/ITAM Positive Feedback Loop. Molecular Cell 10, 1057-1069.

Rowland, S.L., DePersis, C.L., Torres, R.M., and Pelanda, R. (2010). Ras activation of Erk restores impaired tonic BCR signaling and rescues immature B cell differentiation. The Journal of experimental medicine 207, 607-621.

Rowley, R.B., Burkhardt, A.L., Caho, H.-G., Matsueda, G.R., and Bolen, J.B. (1995). Syk protein-tyrosine kinase is regulated by tyrosine-phosphorylated $\lg$ alpha/lg beta immunoreceptor tyrosine activation motif binding and autophosphorylation. The Journal of biological chemistry 270, 11590-11594.

Ruland, J., Duncan, G.S., Elia, A., del Barco Barrantes, Ivan, Nguyen, L., Plyte, S., Millar, D.G., Bouchard, D., Wakeham, A., and Ohashi, Pamela S.: Mak, Tak W. (2001). Bcl10 Is a Positive Regulator of Antigen Receptor-Induced Activation of NF-KB and Neural Tube Closure. Cell 104, 33-42. 
Saijo, K., Mecklenbräuker, I., Santana, A., Leitger, M., Schmedt, C., and Tarakhovsky, A. (2002). Protein Kinase C $\beta$ Controls Nuclear Factor KB Activation in B Cells Through Selective Regulation of the IKB Kinase a. Journal of Experimental Medicine 195, 16471652.

Salim, K., Bottomley, M.J., Querfurth, E., Zvelebil, M.J., Gout, I., Scaife, R., Margolis, R.L., Gigg, R., Smith, C.E., and Driscoll, P.C., et al. (1996). Distinct specificity in the recognition of phosphoinositides by the pleckstrin homology domains of dynamin and Bruton's tyrosine kinase. EMBO Journal 15, 6241-6250.

Sanchez, M., Misulovin, Z., Burkhardt, A.L., Mahajan, S., Costa, T.E., Franke, R.R., Bolen, J.B., and Nussenzweig, M.C. (1993). Signal Transduction by Immunoglobulin Is Mediated Through Ig $\alpha$ and $\lg \beta$. Journal of Experimental Medicine 178, 1049-1105.

Sanjana, N.E., Le Cong, Zhou, Y., Cunniff, M.M., Feng, G., and Zhang, F. (2012). A transcription activator-like effector toolbox for genome engineering. Nature protocols 7 , 171-192.

Saouaf, S.J., Mahajan, S., Rowley, R.B., Kut, S.A., Fargnoli, J., Burkhardt, A.L., Tsukada, S., Witte, O.N., and Bolen, J.B. (1994). Temporal differences in the activation of three classes of non-transmembrane protein tyrosine kinases following B-cell antigen receptor surface engagement. Proc. Natl. Acad. Sci. USA 91, 9524-9528.

Schatz, D.G., and Ji, Y. (2011). Recombination centres and the orchestration of V(D)J recombination. Nature reviews. Immunology 11, 251-263.

Schulze-Luehrmann, J., and Ghosh, S. (2006). Antigen-receptor signaling to nuclear factor kappa B. Immunity 25, 701-715.

Shaw, A.C., Mitchell, R.N., Weaver, Y.K., Campos-Torres, J., Abbas, A.K., and Leder, P. (1990). Mutations of immunoglobulin transmembrane and cytoplasmic domains: Effects on intracellular signaling and antigen presentation. Cell 63, 381-392.

Shinohara, H., Maeda, S., Watarai, H., and Kurosaki, T. (2007). IkB kinase $\beta$-induced phosphorylation of CARMA1 contributes to CARMA1 Bcl10 MALT1 complex formation in B cells. The Journal of experimental medicine 204, 3285-3293.

Shinohara, H., Yasuda, T., Aiba, Y., Sanjo, H., Hamadate, M., Watarai, H., Sakurai, H., and Kurosaki, T. (2005). PKC $\beta$ regulates BCR-mediated IKK activation by facilitating the interaction between TAK1 and CARMA1. The Journal of experimental medicine 202, 1423-1431. 
Shumway, S.D., Maki, M., and Miyamoto, S. (1999). The PEST Domain of IkBa Is Necessary and Sufficient for in Vitro Degradation by $\mu$-Calpain. The Journal of biological chemistry $274,30874-30881$.

Soboloff, J., Rothberg, B.S., Madesh, M., and Gill, D.L. (2012). STIM proteins: dynamic calcium signal transducers. Nature reviews. Molecular cell biology 13, 549-565.

Sohn, H.W., Gu, H., and Pierce, S.K. (2003). Cbl-b negatively regulates B cell antigen receptor signaling in mature $B$ cells through ubiquitination of the tyrosine kinase Syk. The Journal of experimental medicine 197, 1511-1524.

Sommer, K., Guo, B., Pomerantz, J.L., Bandaranayake, A.D., Moreno-García, M.E., Ovechkina, Y.L., and Rawlings, D.J. (2005). Phosphorylation of the CARMA1 linker controls NF-KB activation. Immunity 23, 561-574.

Song, J.J., Kim, J.-H., Sun, B.K., Alcala, M.A., Bartlett, D.L., and Lee, Y.J. (2010). c-Cbl acts as a mediator of Src-induced activation of the PI3K-Akt signal transduction pathway during TRAIL treatment. Cellular signalling 22, 377-385.

Soubeyran, P., Kowanetz, K., Szymkiewicz, I., Langdon, W.Y., and Dikic, I. (2002). CblCIN85-endophilin complex mediates ligand-induced downregulation of EGF receptors. Nature 416, 183-187.

Stamenova, S.D., French, M.E., He, Y., Francis, S.A., Kramer, Z.B., and Hicke, L. (2007). Ubiquitin binds to and regulates a subset of SH3 domains. Molecular Cell 25, 273-284.

Stevens, T.L., Blum, J.H., Foy, S.P., Matsuuchi, L., and DeFranco, A.L. (1994). A Mutation of the $\mu$ Transmembrane That Disrupts Endoplasmic Reticulum Retention. Journal of immunology 152, 4397-4406.

Stoddart, A., Dykstra, M.L., Brown, B.K., Song, W., Pierce, S.K., and Brodsky, F.M. (2002). Lipid Rafts Unite Signaling Cascades with Clathrin to Regulate BCR Internalization. Immunity 17, 451-462.

Stoddart, A., Jackson, A.P., and Brodsky, F.M. (2005). Plasticity of B Cell Receptor Internalization upon Conditional Depletion of Clathrin. Molecular Biology of the Cell 16, 2339-2348.

Su, T.T., Guo, B., Kawakami, Y., Sommer, K., Chae, K., Humphries, L.A., Kato, R.M., Kang, S., Patrone, L., and Wall, R., et al. (2002). PKC- $\beta$ controls IKB kinase lipid raft recruitment and activation in response to BCR signaling. Nature immunology 3, 780-786.

Szymkiewicz, I., Kowanetz, K., Soubeyran, P., Dinarina, A., Lipkowitz, S., and Dikic, I. (2002). CIN85 participates in Cbl-b-mediated down-regulation of receptor tyrosine kinases. The Journal of biological chemistry 277, 39666-39672. 
Takata, M., Sabe, H., Hata, A., Inazu, T., Homma, Y., Nukada, T., Yamamura, H., and Kurosaki, T. (1994). Tyrosine kinases Lyn and Syk regulate B cell receptor-coupled $\mathrm{Ca}^{2+}$ mobilization through distinct pathways. EMBO Journal 13, 1341-1349.

Take, H., Watanabe, S., Takeda, K., Yu, Z.X., Iwata, N., and Kajigaya, S. (2000). Cloning and characterization of a novel adaptor protein, CIN85, that interacts with $\mathrm{c}$-Cbl. Biochemical and biophysical research communications 268, 321-328.

Tanner, M.J., Hanel, W., Gaffen, S.L., and Lin, X. (2007). CARMA1 coiled-coil domain is involved in the oligomerization and subcellular localization of CARMA1 and is required for T cell receptor-induced NF-KB activation. The Journal of biological chemistry 282, 1714117147.

Thien, C., and Langdon, W.Y. (2001). Cbl: many adaptations to regulate protein tyrosine kinases. Nature reviews. Molecular cell biology 2, 294-305.

Thome, M., and Weil, R. (2007). Post-translational modifications regulate distinct functions of CARMA1 and BCL10. Trends in immunology 28, 281-288.

Tibaldi, E.V. (2003). CD2BP3, CIN85 and the structurally related adaptor protein CMS bind to the same CD2 cytoplasmic segment, but elicit divergent functional activities. International Immunology 15, 313-329.

Tompa, P. (2005). The interplay between structure and function in intrinsically unstructured proteins. FEBS letters 579, 3346-3354.

Tossidou, I., Kardinal, C., Peters, I., Kriz, W., Shaw, A., Dikic, I., Tkachuk, S., Dumler, I., Haller, H., and Schiffer, M. (2007). CD2AP/CIN85 balance determines receptor tyrosine kinase signaling response in podocytes. The Journal of biological chemistry 282, 74577464.

Tossidou, I., Niedenthal, R., Klaus, M., Teng, B., Worthmann, K., King, B.L., Peterson, K.J., Haller, H., and Schiffer, M. (2012). CD2AP regulates SUMOylation of CIN85 in podocytes. Molecular and Cellular Biology 32, 1068-1079.

Towbin, H., Staehelin, and Gordon, J. (1979). Electrophoretic transfer of proteins from polyacrylamide gels to nitrocellulose sheets: Procedure and some applications. Proc Natl Acad Sci U S A 76, 4350-4354.

Varnai, P., Rother, K.I., and Balla, T. (1999). Phosphatidylinositol 3-Kinase-dependent Membrane Association of the Bruton's Tyrosine Kinase Pleckstrin Homology Domain Visualized in Single Living Cells. The Journal of biological chemistry 274, 10983-10989.

Venkitaraman, A.R., Williams, G.T., Dariavach, P., and Neuberger, M.S. (1991). The Bcell antigen receptor of the five Immunoglobulin classes. Nature 352, 777-781. 
Vilen, B.J., Nakamura, T., and Cambier, J.C. (1999). Antigen-Stimulated Dissociation of BCR mlg from Ig- $\alpha / \mathrm{lg}-\beta$ : Implications for Receptor Desensitization. Immunity 10, 239-248. Vos, Q., Lees, A., Wu, Z.-Q., Snapper, C.M., and Mond, J.J. (2000). B-cell activation by Tcell-independent type 2 antigens as an integral part of the humoral immune response to pathogenic microorganisms. Immunological reviews 176, 154-170.

Wang, C., Deng, L., Hong, M., Akkaraju, G.R., Inoue, J.-i., and Chen, Z.J. (2001). TAK1 is a ubiquitin-dependent kinase of MKK and IKK. Nature 412, 346-351.

Wang, D., Matsumoto, R., You, Y., Che, T., Lin, X.-Y., Gaffen, S.L., and Lin, X. (2004). CD3/CD28 Costimulation-Induced NF-kB Activation Is Mediated by Recruitment of Protein Kinase $\mathrm{C}-\theta, \mathrm{Bcl} 10$, and IKB Kinase $\beta$ to the Immunological Synapse through CARMA1. Molecular and Cellular Biology 24, 164-171.

Wang, X., Li, J.-P., Kuo, H.-K., Chiu, L.-L., Dement, G.A., Lan, J.-L., Chen, D.-Y., Yang, C.-Y., Hu, H., and Tan, T.-H. (2012). Down-regulation of B cell receptor signaling by hematopoietic progenitor kinase 1 (HPK1)-mediated phosphorylation and ubiquitination of activated B cell linker protein (BLNK). The Journal of biological chemistry 287, 1103711048.

Ward, J.J., Sodhi, J.S., McGuffin, L.J., Buxton, B.F., and Jones, D.T. (2004). Prediction and functional analysis of native disorder in proteins from the three kingdoms of life. Journal of molecular biology 337, 635-645.

Watanabe, S., Take, H., Takeda, K., Yu, Z.X., Iwata, N., and Kajigaya, S. (2000). Characterization of the CIN85 adaptor protein and identification of components involved in CIN85 complexes. Biochemical and biophysical research communications 278, 167-174.

Weber, M., Treanor, B., Depoil, D., Shinohara, H., Harwood, N.E., Hikida, M., Kurosaki, T., and Batista, F.D. (2008). Phospholipase C-y2 and Vav cooperate within signaling microclusters to propagate $B$ cell spreading in response to membrane-bound antigen. The Journal of experimental medicine $205,853-868$.

Weigert, M.G., Cesari, I.M., Yonkovich, S.J., and Cohn, M. (1970). Variability in the Lambda Light Chain Sequences of Mouse antibody. Nature 228, 1045-1047.

Welsch, T., Endlich, N., Gökce, G., Doroshenko, E., Simpson, J.C., Kriz, W., Shaw, A.S., and Endlich, K. (2005). Association of CD2AP with dynamic actin on vesicles in podocytes. American journal of physiology. Renal physiology 289, F1134-43.

Wienands, J., Schweikert, J., Wollscheid, B., Jumaa, H., Nielsen, P.J., and Reth, M. (1998). SLP-65: A New Signaling Component in B Lymphocytes which Requires 
Expression of the Antigen Receptor for Phosphorylation. Journal of Experimental Medicine 188, 791-795.

Winding, P., and Berchtold, M.W. (2001). The chicken B cell line DT40: a novel tool for gene disruption experiments. Journal of Immunological Methods 249, 1-16.

Wright, P.E., and Dyson, H.J. (1999). Intrinsically Unstructured Proteins: Re-assessing the Protein Structure-Function Paradigm. J. Mol. Biol. 239, 321-331.

Wu, C.-J., Conze, D.B., Li, T., Srinivasula, S.M., and Ashwell, J.D. (2006). Sensing of Lys 63-linked polyubiquitination by NEMO is a key event in NF-KB activation [corrected]. Nature cell biology 8, 398-406.

Wu, J.N., and Koretzky, G.A. (2004). The SLP-76 family of adapter proteins. Seminars in Immunology 16, 379-393.

Xue, L., Morris, S.W., Orihuela, C., Tuomanen, E., Cui, X., Wen, R., and Wang, D. (2003). Defective development and function of Bcl10-deficient follicular, marginal zone and B1 B cells. Nature immunology 4, 857-865.

Yokouchi, M., Kondo, T., Sanjay, A., Houghton, A., Yoshimura, A., Komiya, S., Zhang, H., and Baron, R. (2001). Src-catalyzed phosphorylation of c-Cbl leads to the interdependent ubiquitination of both proteins. The Journal of biological chemistry 276, 35185-35193.

Yokoyama, K., Su, I.-h., Tezuka, T., Yasuda, T., Mikoshiba, K., Tarakhovsky, A., and Yamamoto, T. (2002). BANK regulates BCR-induced calcium mobilization by promoting tyrosine posphorylation of $\mathrm{IP}_{3}$ receptor. EMBO Journal 21, 83-92.

Zhang, J., Patel, J.M., and Block, E.R. (1998). Hypoxia-specific upregulation of calpain activity and gene expression in pulmonary artery endothelial cells. American journal of physiology-Lung cellular and molecular physiology 275, L461-L468.

Zhang, J., Zheng, X., Yang, X., and Liao, K. (2009). CIN85 associates with endosomal membranes and binds phosphatidic acid. Cell research 19, 733-746.

Zhang, S.L., Yeromin, A.V., Zhang, X.H.-F., Yu, Y., Safrina, O., Penna, A., Roos, J., Stauderman, K.A., and Cahalan, M.D. (2006). Genome-wide RNAi screen of $\mathrm{Ca}^{2+}$ influx identifies genes that regulate $\mathrm{Ca}^{2+}$ release-activated $\mathrm{Ca}^{2+}$ channel activity. PNAS 103, 9357-9362.

Zhao, J., Bruck, S., Cemerski, S., Zhang, L., Butler, B., Dani, A., Cooper, J.A., and Shaw, A.S. (2013). CD2AP links cortactin and capping protein at the cell periphery to facilitate formation of lamellipodia. Molecular and Cellular Biology 33, 38-47. 
CURRICULUM VITAE 